\title{
Fair allocation and reallocation: an axiomatic study
}

Citation for published version (APA):

Klaus, B. E. (1997). Fair allocation and reallocation: an axiomatic study. [Doctoral Thesis, Maastricht University]. Universiteit Maastricht. https://doi.org/10.26481/dis.19980113bk

Document status and date:

Published: 01/01/1997

DOI:

10.26481/dis.19980113bk

Document Version:

Publisher's PDF, also known as Version of record

\section{Please check the document version of this publication:}

- A submitted manuscript is the version of the article upon submission and before peer-review. There can be important differences between the submitted version and the official published version of record.

People interested in the research are advised to contact the author for the final version of the publication, or visit the DOI to the publisher's website.

- The final author version and the galley proof are versions of the publication after peer review.

- The final published version features the final layout of the paper including the volume, issue and page numbers.

Link to publication

\footnotetext{
General rights rights.

- You may freely distribute the URL identifying the publication in the public portal. please follow below link for the End User Agreement:

www.umlib.nl/taverne-license

Take down policy

If you believe that this document breaches copyright please contact us at:

repository@maastrichtuniversity.nl

providing details and we will investigate your claim.
}

Copyright and moral rights for the publications made accessible in the public portal are retained by the authors and/or other copyright owners and it is a condition of accessing publications that users recognise and abide by the legal requirements associated with these

- Users may download and print one copy of any publication from the public portal for the purpose of private study or research.

- You may not further distribute the material or use it for any profit-making activity or commercial gain

If the publication is distributed under the terms of Article $25 \mathrm{fa}$ of the Dutch Copyright Act, indicated by the "Taverne" license above, 


\title{
Fair Allocation and Reallocation: An Axiomatic Study
}

\author{
Proefschrift
}

Ter verkrijging van de graad van doctor aan de Universiteit Maastricht, op gezag van de Rector Magnificus, Prof.dr. A.C. Nieuwenhuijzen Kruseman, volgens het besluit wan het College van Decanen,

in het openbaar te verdedigen op dinsdag 13 januari 1998 om 12.00 uur

door

Bettina-Elisabeth Klaus 
Promotor: Prof.dr. H.J.M. Peters

Copromotor: Dr. A.J.A. Storcken

Beoordelingscommissie:

Prof.dr. S.H. Tijs (Universiteit Maastricht en Katholieke Universiteit Brabant, voorzitteri) Dr. J.J.M. Derks.

Prof.dr. L. Gevers (Facultés Universitaires Notre-Dame de la Paix, Namur)

Prof.dr. P.H.M. Ruys (Katholieke Uniwersiteit Brabant)

Dr.ir. J. wan der Stel

Prof.dr. W. Thomson (University of Rochester, USA)

ISBN 90-5681-027-8 


\section{Acknowledgements}

I wish to thank all persons who helped me either directly or indirectly to accomplish this dissertation.

First of all I like to thank Hans Peters and Ton Storcken for their support throughout my entire $\mathrm{Ph}$.D.-student period. They not only showed me the first steps in economics, but they were always willing to help me while I was learning how to walk on scientific ground. After I promenaded under their supervision in Maastricht for three years, they had enough confidence in my walking skills to let me walk into the wide world.

As a visiting scholar I only wanted to spend four months at the University of Rochester, USA. The four months became a whole year and I wish to thank the Department of Economics at the University of Rochester for their hospitality. In particular, I wish to thank William Thomson, not only for inviting me to come to Rochester, but also for the interest he took in my research and the many discussions that broadened my view and helped me improve my exposition. It is in Rochester that I realized that I not only want to walk on economic ground, but that, in fact, I want to run.

I would like to thank my colleagues in Maastricht and Rochester for numerous stimulating discussions and the good times we had together. In particular, I thank Andres Perea y Monsuwé for sharing the office with a somewhat chaotic person; Andre Hoogstrate, Hans Peters, and Horst Zank for helping me with bureaucratic and organizational chores while I was in Rochester; Arie Koster for taking care of my apartment and plants during my absence; and Richard Orrell for reading parts of my thesis and giving his opinions as a non-economist.

I am grateful to the Netherlands Organization of Scientific Research (NWO) for the financial support that allowed me to stay at the University of Rochester during the spring semester 1997. I also wish to thank Shell Nederland N.V. for a travel grant that allowed me to present my work at the International Conference on Game Theory and Economic Applications 1996 in Bangalore, India. 
"Alles, was Gegenstand des wissenschaftlichen Denkens überhaupt sein kann, verfällt, sobald es zur Bildung einer Theorie reif ist, der axiomatischen $\mathrm{Me}$ thode und damit mittelbar der Mathematik."1

David Hilbert (1918)

\footnotetext{
${ }^{1}$ Everything, which may passibly be subject to scientific thought, as soon as it is ready for the development of a theory, comes within the scope of the axiomatic method and thus becomes treatable by means of mathematics.

This translation into English is borrowd from the Introduction to the book "Mathematical Economics" Twenty Papers of Gerard Debreu", Econometric Society Monographs No. 4, ed. Frank Halln, Cambridge University Press, 1983.
} 


\section{Contents}

$\begin{array}{ll}\text { Notation } & 5\end{array}$

1 Introduction $\quad 7$

1.1 Strategy-proofness and single-peakedness .............. . . . 7

1.2 Fair allocation and single-peakedness . . . . . . . . . . . . . . . 8

1.3 Fair allocation and single-dippedness . . . . . . . . . . . . . . 10

1.4 Fair reallocation and single-peakedness . . . . . . . . . . . . . 11

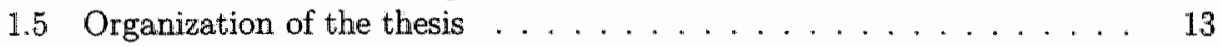

I Fair allocation $\quad 15$

2 Fair allocation and single-peakedness $\quad 17$

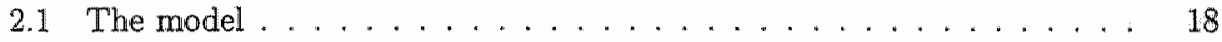

2.2 The uniform rule . . . . . . . . . . . . . . . . . . . . 21

2.3 The independence of replication-invariance . . . . . . . . . . . . 29

3 Fair allocation and single-dippedness 35

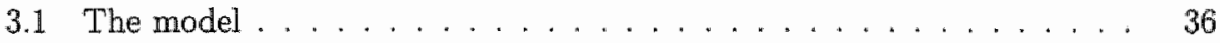

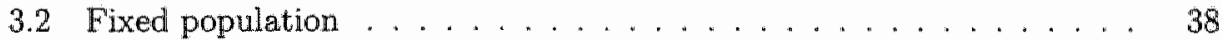

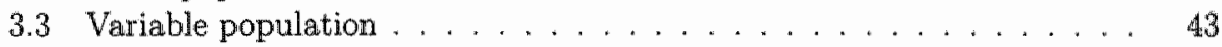

3.4 Independence and compatibilities ................. 50

II Fair reallocation $\quad \mathbf{5 5}$

4. The uniform reallocation rule $\quad 57$

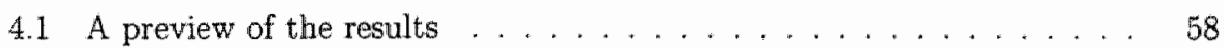

4.2 The model . . . . . . . . . . . . . . . . . . . . . 61

4.3 Strategy-proofness, no-envy, and peak-onliness .......... 66

4.3.1 Strategy-proofness and nomenvy ............... 66

4.3.2 Endowment strategy-proofness . . . . . . . . . . . . 73

4.3.3 Demand-onliness, reversibility, and peak-onliness . . . . . . 76

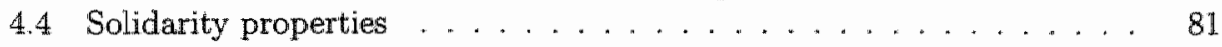

4.4.1 One-sided population-monotonicity ............ 82 
4.4 .2 One-sided replacement-domination $\ldots \ldots \ldots \ldots \ldots . \ldots . \ldots$

4.4 .3 One-sided endowment-monotonicity . . . . . . . . . 88

4.5 Consistency . . . . . . . . . . . . . . . . . . . . . 97

4.6 Proofs of independence . . . . . . . . . . . . . 104

5 Model variations $\quad 109$

5.1 Strategy-proof reallocation with debts . . . . . . . . 110

5.2 Strategy-proof reallocations without debts . . . . . . . . 115

5.3 Further model variations . . . . . . . . . . . . . . . . 118

5.3 .1 Discontinuous preferences . . . . . . . . . . . 118

5.3.2 Bounded peaks, individual endowments, and reallocations ... 118

5.3 .3 Finite potential set of agents . . . . . . . . . . . . . 119

Appendix A: Allocation of an indivisible good 121

$\begin{array}{lr}\text { Appendix B: Two alternative proofs } & 125\end{array}$

$\begin{array}{ll}\text { References } & 127\end{array}$

Index 131

Author Index . . . . . . . . . . . . . . . . . . . . . . . . . 132

Subject Index . . . . . . . . . . . . . . . . . . . . . . 134

List of Symbols . . . . . . . . . . . . . . . . . . 136

Summary in Dutch 137

$\begin{array}{ll}\text { Curriculum Vitae } & 141\end{array}$ 


\section{List of Tables}

2.1 Characterizations of the uniform rule $U$ : Pareto optimality and strategyproofness. . . . . . . . . . . . . . . . .

2.2 Characterizations of the uniform rule $U$ : Pareto optimality, no-envy, and solidarity properties. . . . . . . . . . . . 26

2.3 Characterizations of the uniform rule $U$ : Pareto optimality, consistency properties, etc. . . . . . . . . . . . . 28

2.4 Characterizations of the uniform rule $U$ without Pareto optimality. . . 29

4.1 Characterizations of the uniform rule $U^{r}$ : Pareto optimality and strategyproofness properties. . . . . . . . . . . . 81

4.2 Characterizations of the uniform rule $U^{r}$ : Pareto optimality, no-envy and solidarity properties. . . . . . . . . . . . . . 9 97

4.3 Characterizations of the uniform rule $U^{\text {r }}$ : Pareto optimality, bilateral consistency etc. . . . . . . . . . . . . . . . . . 104

4.4 Proofs of independence, Section $4.3 \ldots \ldots \ldots \ldots . \ldots 106$

4.5 Proofs of independence, Section $4.4 \ldots \ldots \ldots \ldots$. . . . . . 107

4.6 Proofs of independence, Section $4.5 \ldots \ldots \ldots \ldots$ 


\section{Notation}

Many of the notation we use throughout the thesis are defined in the text were they appear for the first time. Here, we give a short list of some notational conventions we do not introduce explicitly again.

\section{Set theoretical notations}

Let $A, B$ be sets.

$A \subseteq B \quad A$ is a subset of $B$.

$A \subsetneq B \quad A$ is a strict subset of $B$, i.e., $A \subseteq B$ and $A \neq B$.

$|A| \quad$ cardinality of $A$.

\section{Natural and real numbers}

$\mathbb{N} \quad$ set of natural numbers.

$\mathbb{Q}$ set of rational numbers.

$\mathbb{R} \quad$ set of real numbers.

$\mathbb{R}_{+} \quad:=\{x \in \mathbb{R} \mid x \geq 0\}$, set of nonnegative real numbers.

$\mathbb{R}^{N}, \mathbb{R}_{+}^{N}$ Cartesian product of $|N|$ copies of $\mathbb{R}$, or $\mathbb{R}_{+}$ respectively, indexed by the elements of $N$. 
Let $a, b \in \mathbb{R}, a \leq b$, and $N \subseteq \mathbb{N}, N$ finite.

$$
\begin{aligned}
(a, b) & :=\{x \in \mathbb{R} \mid a<x<b\}, \\
& \text { open interval between } a \text { and } b . \\
{[a, b] } & :=\{x \in \mathbb{R} \mid a \leq x \leq b\} \\
& \text { closed interval between } a \text { and } b . \\
(a, b] & :=\{x \in \mathbb{R} \mid a<x \leq b\}, \\
{[a, b) } & :=\{x \in \mathbb{R} \mid a \leq x<b\} \\
& \text { half-open intervals between } a \text { and } b .
\end{aligned}
$$

\section{Miscellaneous}

Let $x, y \in \mathbb{R}^{N}$.

$x \leq y \quad$ for all $i \in N, x_{i} \leq y_{i}$.

$\sum_{N} x_{i}:=\sum_{i \in N} x_{i}$

$\infty$ infinity.

- end of a proof.

$\diamond \quad$ end of an example.

$\square \quad$ end of a remark. 


\section{Chapter 1}

\section{Introduction}

\subsection{Strategy-proofness and single-peakedness}

In many economic and political decision situations the preferences of the agents involved are private information. Depending on the decision mechanism to be used, agents may have an incentive to strategically misrepresent their preferences in order to influence the final outcome to their own advantage, probably at the expense of others. Furthermore, misrepresentation of preferences may yield an outcome that is Pareto-inferior with respect to the true preferences.

Misrepresentation of preferences can be avoided by imposing strategy-proofness on the mechanism. A mechanism (for instance, a voting procedure) is strategy-proof if no agent can ever gain by misrepresenting his preferences, irrespective of the preferences announced by the other agents. An additional advantage of strategy-proofness is that it simplifies the application of the mechanism, because every agent only needs to know his own preference relation in order to determine his optimal strategy.

A first fundamental result concerning strategy-proofness was stated by Gibbard (1973) and Satterthwaite (1975) for the following abstract social choice model. The set of alternatives is fnite and all strict preferences over the set of alternatives are admissible. Then, a mechanism with at least three alternatives in its range is strategy-proof if and only if it is dictatorial. However, non-dictatorial strategy-proof mechanisms exist on certain "restricted domains" such as the well-known domain of "single-peaked" preferences: preferences are single-peaked if alternatives can be ordered in such a way that every agent has a single best alternative, his peak, and his welfare decreases monotonically in either direction away from his peak. Single-peaked preferences were introduced by Black (1948) in an election context. For quite some time, contributions to the anal= ysis of strategy-proof mechanisms for single-peaked preferences were concerned mainly with public goods. See, for instance, Dummett and Farquharson (1961), Blin and Satterthwaite (1976), Moulin (1980), Border and Jordan (1983), and Barbera, Gul, and Stacchetti (1993).

In this thesis, we consider several private good models of allocating, or reallocating, an infinitely divisible commodity among a group of agents. Again, strategy-proofness will be a central property in our analysis. However, we are also interested in properties 
that express some notion of faimess or solidarity, for instance, no-envy or populationmonotonicity. No-envy requires that no agent prefers the share of another agent to his own share. Population-monotonicity requires that after the arrival of new agents, either all agents initially present (weakly) lose together or they all (weakly) gain together. In the sequel, we refer to the analysis of allocation or reallocation mechanisms that satisfy properties of fairness and other "desirable" properties as "fair allocation", or "fair reallocation" respectively.

\subsection{Fair allocation and single-peakedness ${ }^{1}$}

The literature on strategy-proofness for private good situations also starts off with an impossibility result. For two-agent two-good exchange economies, Hurwicz (1972) showed that even for a restricted domain that contains all translated Cobb-Douglas preferences, essentially no allocation mechanism satisfies strategy-proofness and Pareto optimality. Similar to the public good case, this incompatibility does not occur on some other restricted domains. For instance, there are many situations where preferences are monotonic and the class of mechanisms that satisfy desirable properties from our normative point of view is rich. Examples are bankruptcy (O'Neill, 1982; Aumann and Maschler, 1985), taxation (Young, 1987), and cost and surplus sharing (Moulin, 1987 and 1997).

Another well-known restricted domain for many allocation problems is the domain of single-peaked preferences. A typical example is rationing in a two-good exchange economy when prices are in disequilibrium (see Benassy, 1982, and Barberà and Jackson, 1995). If preferences over the two-dimensional space of bundles are strictly convex, then the restrictions of these preferences to the budget lines are single-peaked. Rationing one of the goods then implies an allocation of the other good, in view of fixed prices and budget constraints. In this context of strategy-proof exchange, Benassy (1982) discussed what is now known as the "uniform rule". It divides the amount that has to be allocated as equally as possible, taking the peaks of the agents as upper bounds in case of excess demand and as lower bounds in case of excess supply. This rule also applies to the problem of allocating a social endowment of some perfectly divisible commodity among a group of agents with single-peaked preferences. Consider the following example.

Example 1.1 Allocation of teaching hours: A fixed number of teaching hours has to be distributed among the members of a university department. Each member has a preferred number of teaching hours, above which and below which his welfare is decreasing. However, the optimal shares, or peaks, might add up to more (excess demand) or to less (excess supply) than the required total teaching load. Given that we have either excess demand or excess supply, the question we ask is: What is a "fair allocation" of the teaching load?

To be more specific, assume that in the department four staff members, Professors $A, B, C$, and $D$, have to share 20 hours of teaching per week. Professor $A$ would

\footnotetext{
${ }^{1}$ See also the introduction of Chapter 2.
} 
like to teach for 3 hours, Professor $B$ 's optimal share is equal to 5 hours, Professor $C$ does not want to teach at all, in contrary to his colleague Professor $D$ who wishes to teach 7 hours. We assume that the Professors' welfares decrease with the distance from their optimal shares. Formally, we denote this allocation problem by the group of Professors $N=\{A, B, C, D\}$ and their peak vector $p(R)=(3,5,0,7)$. Note that this is an allocation problem with excess supply.

First, we consider equal division: the allocation of the teaching load equals $(5,5,5,5)$. However, it is easy to see that this allocation is not Pareto optimal since Professors $A$ and $D$ can reach their peaks by transferring two hours from Professor $A$ to Professor $D$.

Dividing the 20 hours of teaching in proportion to the peaks yields the following allocation: $\left(4,6 \frac{2}{3}, 0,9 \frac{1}{3}\right)$. Now, one can argue whether it is fair that everyone but Professor $C$ has to work more than their optimal amount. Furthermore, it is likely that Professor $D$ prefers to teach $6 \frac{2}{3}$ hours, the amount that is assigned to his colleague $B$, than the $9 \frac{1}{3}$ hours that were assigned to him. In that case, he envies Professor $B$, a bad thing for the atmosphere of the department. Besides the fact that the proportional allocation does not satisfy no-envy, it is easy to show that it is not strategy-proof. If, for instance, Professor $D$ declares that not 7 , but $5 \frac{1}{4}$ is his optimal amount, then he profits from lying and receives as a proportional share his optimal amount of teaching.

Next, we consider the division of the 20 hours of teaching according to the uniform rule we mentioned above, i.e., we divide the teaching hours as equally as possible, taking the peaks of the Professors as lower bounds. We briefly describe how this allocation can be constructed. First, we endow all Professors with their optimal teaching shares $(3,5,0,7)$. Now, we still have to allocate 5 hours of teaching. We start to increase the teaching load of the Professor with the smallest amount to teach until either his teaching load is equal to that of the Professor with the second lowest teaching load or we have assigned all teaching hours. This yields $(3,5,3,7)$. Now, there remain 2 hours of teaching. Again, we increase the teaching load of the Professors with the smallest teaching load until they have a teaching load equal to that of the Professor with the new second lowest teaching load or until we have assigned all teaching hours. This yjelds the allocation $(4,5,4,7)$ and we are done. Note that this allocation is Pareto optimal and there is no envy between the Professors.

The following example demonstrates the computation of the uniform allocation for an excess demand allocation problem.

Example 1.2 Beer allocation: At a party there are 5 liters of beer left and the remaining guests $N=\{\mathrm{a}, \mathrm{b}, \mathrm{c}, \mathrm{d}, \mathrm{e}\}$ still wish to consume $p(R)=\left(2, \frac{1}{2}, 1,2,3\right)$ liters. If free disposal is not allowed, then preferences over the consumption of beer are likely to be single-peaked. Since the sum of the peaks is greater that the amount of beer that is left, we have an allocation problem with excess demand. Now the uniform allocation of this problem can be determined as follows. We start dividing the beer equally until either one of the guests reaches his peak or we have divided all the beer. This yields the allocation $\left(\frac{1}{2}, \frac{1}{2}, \frac{1}{2}, \frac{1}{2}, \frac{1}{2}\right)$. Now, we still have to allocate $2 \frac{1}{2}$ liters of beer. We start 
to increase the amount of beer of all guests who still want more equally until, again, either one of these guests reaches his peak or we have divided all the beer. This yields the allocation $\left(1, \frac{1}{2}, 1,1,1\right)$ Now, we still have to allocate $\frac{1}{2}$ liters of beer. We repeat this procedure once more and finally get the allocation $\left(1 \frac{1}{6}, \frac{1}{2}, 1,1 \frac{1}{5}, 1 \frac{1}{6}\right)$. Note that this allocation again is Pareto optimal and there is no envy between the guests.

It turns out that the uniform rule we applied in Examples 1.1 and 1.2 for the allocation of teaching hours and of beer not only satisfies many desirable properties for these specific examples, but for all possible allocation problems. Sprumont (1991) proved that the uniform rule is the only rule that satisfies Pareto optimality, strategy-proofness, and either no-envy or anonymity. The last condition means that an agent's share does not depend on his name. Ching (1994) weakens anonymity to a condition called equal treatment of equals: agents with the same preferences are treated equally. The uniform rule satisfies many other desirable conditions, such as consistency, solidarity, and monotonicity properties. See Thomson (1994a,b, 1995a, 1997a), Sönmez (1994), and Dagan (1995). Further characterizations where obtained by De Frutos and Massó (1994) using a condition of Lorenz maximality, and Otten, Peters, and Volij (1995), applying conditions from bargaining theory. As a conclusion of this research the uniform rule is now accepted as the most important rule for the problem of fair allocation when preferences are single-peaked.

In Chapter 2, we give a review of the most important results concerning this rule. Furthermore, we present the answers to two open questions raised by William Thomson in connection with two characterizations of the uniform rule (Thomson 1995a, 1997a): by means of a single example, we give a positive answer to the question whether replication-invariance in these characterizations is independent from the other characterizing properties.

Proceeding from Chapter 2, we consider two variations of the "classical" allocation problem with single-peaked preferences. In the first variation, which we discuss in the next section, we consider the problem of fair allocation for a class of preferences that can be described as "reversed single-peaked" preferences, or "single-dipped" preferences: preferences are single-dipped if alternatives can be ordered in such a way that every agent thas a single worst alternative, his dip, and this welfare increases monotonically in either direction away from the dip. In the second variation of the allocation problem with single-peaked preferences, we assume that instead of a social endowment that has to be allocated, agents have individual endowments that have to be reallocated.

\subsection{Fair allocation and single-dippedness ${ }^{2}$}

In Chapter 3, we analyze the problem of fair allocation under the assumption that preferences are single-dipped instead of single-peaked. An example of single-dipped preferences are preference relations that can be represented by a strictly convex utility function. Consider, for instance, the following work allocation problem.

See also the introduction of Chapter 3. 
Example 1.3 A university department wants to hire at least one of the former Ph.D. students of the department to do a certain amount of teaching. The former Ph.D. students that are available are unemployed and still receive reduced pay from the university. ${ }^{3}$ Payment is in proportion to the amount of teaching that is done. However, all extra money that is earned is subtracted from the reduced pay the university provides. Under this assumption, the $\mathrm{Ph} . \mathrm{D}$. students (they enjoy their leisure time very much) have single-dipped preferences over the teaching load.

Yet another example is a two-goods exchange economy with fixed prices and strictly quasi-convex utility functions, which induce single-dipped preferences on the budget lines.

Although the domain of single-dipped preferences is less well known than the domain of single-peaked preferences, there are interesting economic situations with underlying single-dipped preferences. For single-dipped preferences in a public good context, we refer to Vickrey (1960), Inada (1964), and Peremans and Storcken (1997). To the best of our knowledge, single-dippedness has only been considered for public good situations.

Assuming for the problem of fair allocation that preferences are single-dipped instead of single-peaked changes the results significantly. It turns out that one of our most important properties, namely Pareto optimality, is not compatible with properties of fairness such as anonymity and no-envy. Furthermore, we show that we must allocate the whole social endowment to a single agent in order to satisfy Pareto optimality, strategy-proofness, and either consistency or one of the solidarity conditions population-monotonicity and "replacement-domination". In addition, we give a full characterization of all allocation rules satisfying the previous combinations of properties. Remarkably, these characterizations are also valid for economies where one indivisible good has to be allocated among a group of agents.

In terms of Example 1.3 we can conclude that only one of the former Ph.D. students is hired to do the teaching job. The question, how this Ph.D. student has to be chosen is answered in Chapter 3.

\subsection{Fair reallocation and single-peakedness ${ }^{4}$}

Another natural variation of the problem of fair allocation with single-peaked preferences is an allocation, or reallocation, of individual endowments. By way of example, consider again the allocation of teaching hours among the members of a university department as described in Example 1.1. Suppose that the allocation was made for the past term, but that before the next term starts, a reallocation is desirable because preferences have changed over time.

Example 1.4 Reallocation of teaching hours: Consider the same university department as in Example 1.1, but now assume that there is an existing distribution of

\footnotetext{
${ }^{3}$ In The Netherlands, unemployed Ph.D. students receive reduced pay, or "wachtgeld" in Dutch, for a certain period after their Ph.D. contract expired.

${ }^{4}$ See also the introduction of Chapter 4.
} 
teaching hours: Again there are four staff members, $N=\{A, B, C, D\}$, who share 20 hours of teaching per week. The individual endowments of teaching from the last term are described by the vector $\omega=(4,6,2,8)$. The preferred teaching load per week the professors announce for the next term is $p(R)=(6,4,6,0)$. Obviously, the existing distribution of teaching hours is not Pareto optimal with respect to the newly announced optima. Hence, by trade all professors could benefit. To be continued.

In economic problems such as the exchange problem mentioned in Section 1.2, individual endowments arise naturally. In Example 1.4, clearly the individual endowments play a crucial role since they discriminate between agents. Another interpretation of this extension can be found in Barberì, Jackson, and Neme (1997), who proceed from the original allocation model with single-peaked preferences and discard anonymity by introducing natural claims or priorities of the agents, for instance in investment situations.

Proceeding from the results we discussed for the original model of fair allocation, we are concerned with the axiomatic analysis of fair reallocation and particularly with an extension of the uniform rule to the reallocation model, the so-called uniform reallocation rule. We revisit Example 1.4.

Example 1.5 Reallocation of teaching hours: Consider the reallocation problem described in Example 1.4 where Professors $A, B, C$, and $D$ with peak vector $p(R)=(6,4,6,0)$ have to reallocate the individual teaching endowments $\omega=(4,8,2,6)$. Hence, in this situation where the sum of the peaks is smaller than the total sum of the individual teaching endowments, Professors $A$ and $C$ who want to teach more in the next term can help to improve the situation by taking some of the teaching load of Professors $B$ and $D$. We are interested in a reallocation of the form $(6, \cdot, 6, \cdot)$. The question is, how to subtract the 6 hours of teaching $A$ and $C$ then receive from $B$ and $D$. Note that the Professors $B$ and $D$ wish to supply 4 and 6 hours of teaching.

Subtracting in proportion to these supplies yields $\left(6,5 \frac{3}{5}, 6,2 \frac{2}{5}\right)$ where Professor $B$ supplies $2 \frac{2}{5}$ and Professor $D$ supplies $3 \frac{3}{5}$ hours of teaching. However, at this reallocation, Frofessor $B$ would prefer to supply $3 \frac{3}{5}$ hours, the amount that his colleague $D$ supplies, instead of the $2 \frac{2}{5}$ hours that he is currently supplying. In this situation, he envies Professor $D$, again, a bad thing for the atmosphere of the department.

Subtracting the 6 hours equally from the individual endowments of the Professors $B$ and $D$ with the peaks as lower bounds, yields the following uniform reallocation $(6,5,6,3)$.

The following example demonstrates the computation of the uniform reallocation for an excess demand reallocation problem.

Example 1.6 Beer reallocation: At a party there are five guests $N=\{\mathrm{a}, \mathrm{b}, \mathrm{c}, \mathrm{d}, \mathrm{e}\}$ who are each endowed with 1 liter of beer. The amounts of beer they wish to consume are $p(R)=\left(0,0,1 \frac{1}{2}, 2,3\right)$ liters. Since the sum of the peaks is greater that the amount of beer that is left, we have a reallocation problem with excess demand. Now the uniform reallocation of this problem can be determined as follows. Guests $a$ and $b$ receive no 
beer and we start dividing their two liters of beer equally among the remaining guests. We do so until either one of the guests reaches his peak or we have divided all the beer. This yields the reallocation $\left(0,0,1 \frac{1}{2}, 1 \frac{1}{2}, 1 \frac{1}{2}\right)$. Now, we still have to allocate $\frac{1}{2}$ liter of beer. We start to increase the amount of beer of all guests who still want more equally until, again, either one of these guests reaches his peak or we have divided all the beer. This yields the allocation $\left(0,0,1 \frac{1}{2}, 1 \frac{3}{4}, 1 \frac{3}{4}\right)$ and we are done.

Similar to the uniform allocation rule, the uniform reallocation rule satisfies many desirable properties such as Pareto optimality, strategy-proofness, and nowenvy in terms of net changes of the endowments.

In Chapters 4 and 5 we answer the question to what extent the results as achieved for the allocation model can be generalized to the more general reallocation model. In Chapter 4 we introduce and explore the reallocation model and properties for realloca tion rules. Furthermore we derive several characterizations of the uniform reallocation rule and discuss the parallels and the differences to related allocation results. Finally, in Chapter 5 , we analyze some variations of the model parameters. We show, for instance, that most of the results remain true if we allow agents to have debts.

\subsection{Organization of the thesis}

Part I of this thesis is concerned with the model of fair allocation we introduced in Sections 1.2 and 1.3. Chapter 2 is devoted to the problem of fair allocation when preferences are single-peaked. Chapter 3 is devoted to the problem of fair allocation when preferences are single-dipped.

Part II is concerned with the model of fair reallocation we introduced in Section 1.4. The main analysis takes place in Chapter 4. In Chapter 5, we consider some model variations and their impact on the results of Chapter 4.

Chapters 2,3 , and 4 are self-contained. Since Chapter 5 is closely related to Chapter 4, it is only" "partly self-contained".

Since the aim of this thesis is an axiomatic analysis of allocation and reallocation models, throughout the remainder of the thesis, axioms, or properties, are typed in slanted. 
Part I

\section{Fair allocation}




\section{Chapter 2}

\section{Fair allocation and single-peakedness ${ }^{1}$}

We consider the allocation, or division, of some perfectly divisible commodity among a group of agents with single-peaked preferences. This means that eacli agent has a most preferred share, his peak, below which and above which his welfare is decreasing. A typical example is rationing in a two-good exchange economy when prices are in disequilibrium (e.g., Benassy 1982): if the preferences of the agents over the two-dimerisional space of bundles are strictly convex, then the restrictions of these preferences to the budget lines are single-peaked. In this context Benassy (1982) considered the so-called uniform rationing scheme. For the more general class of allocation problems with singlepeaked preferences, this solution is known as the uniform rule.

Sprumont (1991) initiated the axiomatic analysis of this class of problems and proved that the uniform rule is the only allocation rule that satisfies Pareto optimality, anonymity, and strategy-proofness. In a second characterization he showed that anonymity can be replaced by no-envy. Since then, a wide literature has been conw cerned with the search for rules with appealing properties. An important conclusion of this research is that the uniform rule is now accepted as the most important rule for allocation problems with single-peaked preferences. It is an extension of this rule to allocation problems when agents have individual endowments, the so-called uniform reallocation rule, that we study extensively in the second part of the thesis. Before we do so, however, we give a short review of the most important results concerning the uniform rule.

This chapter is organized as follows. In Section 2.1 we introduce the model and two allocation rules that capture different notions of fairness: the proportional and the uniform rule. Since the uniform rule turns out to be the central allocation rule when preferences are singlempeaked, in Section 2.2 we introduce several of its properties. Furthermore, we give an overview of the most important characterizations of this rule. ${ }^{2}$

\footnotetext{
This chapter is mainly based on the following papers: Ching (1992, 1994), Dagan (1996), Klaus (1997a), Sönmez (1994), Sprumont (1991), and Thomson (1994a, b, 1995a, 1997a,b).

${ }^{2}$ We refer to these characterizations in Part II where we extend the model to situations where agents have individual endowments and axciomatically study so-called reallocation rules. It turns out that in
} 
Finally, in Section 2.3 we give the answers to two open questions raised by William Thomson in connection with two characterizations of the uniform rule (Thomson 1995a, 1997a): by means of a single example, we give a positive answer to the question whether "replication-invariance" in these characterizations is independent from the other characterizing properties.

\subsection{The model}

There is an infinite population of potential agents, indexed by the natural numbers $\mathbb{N}$. Each agent $i \in \mathbb{N}$ is equipped with a continuous and single-peaked preference relation $R_{i}$ defined over the non-negative real numbers $\mathbb{R}_{+}$. Single-peakedness of $R_{i}$ means that there exists a point $p\left(R_{i}\right) \in \mathbb{R}_{+}$, called the peak of agent $i_{\text {; }}$ with the following property: for all $x, y \in \mathbb{R}_{+}$with $x<y \leq p\left(R_{i}\right)$ or $x>y \geq p\left(R_{i}\right)$, we have $y P_{i} x^{3}$. Each preference relation $R_{i}$ can be described in terms of the preference function $r_{i}: \mathbb{R}_{+} \rightarrow \mathbb{R}_{+} \cup\{\infty\}$ that assigns to each point $x \in \mathbb{R}_{+}$the point on the other side of $p\left(R_{i}\right)$ that is indifferent to $x$, if such a point exists, and otherwise 0 or $\infty$, depending on which side of the peak $x$ is. So, for all $x \in \mathbb{R}_{+}$the following holds.

$$
\begin{aligned}
& \text { If } x \leq p\left(R_{i}\right), \text { then } r_{i}(x) \geq p\left(R_{i}\right) \text { and } \begin{cases}r_{i}(x) I_{i} x & \text { if such a point } \\
r_{i}(x)=\infty & \text { exists, or }\end{cases} \\
& \text { If } x \geq p\left(R_{i}\right), \text { then } r_{i}(x) \leq p\left(R_{i}\right) \text { and } \begin{cases}r_{i}(x) I_{i} x & \text { if such a point } \\
r_{i}(x)=0 & \text { exists, or }\end{cases}
\end{aligned}
$$

By $\mathcal{R}$ we denote the class of all continuous, single-peaked preference relations over $\mathbb{R}_{*}$ and by $\mathcal{R}_{b} \subseteq \mathcal{R}$ the subclass of preference relations such that for all $R_{i} \in \mathcal{R}_{b}$, the corresponding preference function $r_{i}$ is bounded, i.e., $r_{i}(0)<\infty$. We call the elements of $\mathcal{R}_{b}$ bounded. By $\mathcal{N}$ we denote the class of non-empty and finite subsets of $\mathbb{N}$. For $N \in \mathcal{N}, \mathcal{R}^{N}$ denotes the set of (preference) profiles $R=\left(R_{i}\right)_{i \in \mathbb{N}}$ such that for all $i \in N$, $R_{i} \in \mathcal{R}_{;} \mathcal{R}_{b}^{N}$ has a similar meaning. For profile $R \in \mathcal{R}^{N}, p(R)=\left(p\left(R_{i}\right)\right)_{i \in N}$ denotes the corresponding vector of peoks.

Now, an econorny is formalized as follows. Let $\Omega \in \mathbb{R}_{+}$be the amount of an infinitely divisible commodity, or the (social) endowment, that has to be distributed among a group of agents $N \in \mathcal{N}$ with profile $R \in \mathcal{R}^{N}{ }^{4}$ We call a pair $e=(R, \Omega) \in \mathcal{R}^{N} \times \mathbb{R}_{+}$an economy. Let $\mathcal{E}^{N}=R^{N} \times \mathbb{R}_{+}$be the class of all economies. Similarly, let $\mathcal{E}_{b}^{N}=\mathcal{R}_{b}^{N} \times \mathbb{R}_{+}$. A feasible allocation for $e=(R, \Omega) \in \mathcal{E}^{N}$ is a vector $x \in \mathbb{R}_{+}^{N}$ such that $\sum_{N} x_{i}=\Omega$. An

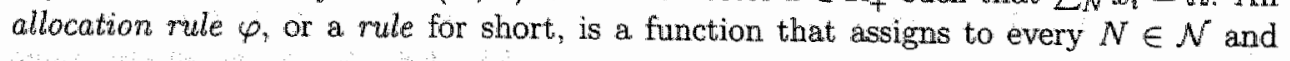
this model of fair reallocation, many results of the allocation model we review in this chapter can be
extended.

${ }^{3}$ As usual, $x R_{i i} y$ is interpreted as " $x$ is weakly preferred to $y^{\prime \prime}$, and $x P_{i} y$ as " $x$ is strictly preferred to $y^{\prime \prime}$. Furthermore, $x I_{i} y$ means that agent $i$ is indifferent between $x$ and $y$.

${ }^{4}$ Note that free disposal of the commodity is not allowed. 
every $e \in \mathcal{E}^{N}$ a feasible allocation, denoted $\varphi(e) .{ }^{5}$ Given $i \in N$, we call $\varphi_{i}(e)$ the share of agent $i$.

A standard requirement for rules is Pareto optimality: an allocation assigned by the rule cannot be changed in such a way that no agent is worse off and some agent is better off.

Pareto optimality: For all $N \in \mathcal{N}$ and all $e=(R, \Omega) \in \mathcal{E}^{N}$, there is no feasible allocation $x \in \mathbb{R}_{+}^{N}$, such that for all $i \in N, x_{i} R_{i} \varphi_{i}(e)$, and for some $j \in N, x_{j} P_{j j} \varphi_{j}(e)$.

By the single-peakedness of the preferences it is easy to show that a rule is Pareto optimal if and only if it is same-sided, that is: for all $N \in \mathcal{N}$ and all $e=(R, \Omega) \in \mathcal{E}^{N}$, either [for all $\left.i \in N, \varphi_{i}(e) \leq p\left(R_{i}\right)\right]$ or [for all $i \in N, \varphi_{i}(e) \geq p\left(R_{i}\right)$ ]. Sprumont (1991) uses same-sidedness as definition of Pareto optimality.

The following two rules embody two different well-known notions of fairness and equity in the literature on fair division, namely the principles of proportionality and of equality.

Young (1988), who investigated the problem of fair division in taxation situations, quotes Aristotle, Ethics, Book V, as follows. "What is just ... is what is proportional and what is unjust is what violates the proportion." In our model, the rule that incorporates Aristotle's notion of justice is the proportional rule.

Proportional rule $P r$ : For all $N \in \mathcal{N}$ and all $e=(R, \Omega) \in \mathcal{E}^{N}$,

$$
\operatorname{Pr}(e):= \begin{cases}\left(\frac{\Omega}{|N|}, \ldots, \frac{\Omega}{|N|}\right) & \text { if } p(R)=(0, \ldots, 0) \in \mathbb{R}^{N}, \\ \lambda p(R) & \text { otherwise, }\end{cases}
$$

where $\lambda \in \mathbb{R}_{+}$solves $\sum_{N} P r_{i}(e)=\Omega$.

The next well-known principle of equity we focus on is equal division. However, taking the single-peakedness of the preferences into account, it turns out that the "equal division allocation" might not be Pareto optimal. In our opinion, the following rule incorporates the idea of equal division under the additional assumption of Pareto optimality: the amount to divide is distributed as equally as possible with all agents' peaks as either lower or upper bounds, depending on whether there is more or less to distribute than the sum of the peaks.

Uniform Rule $U$ : For all $N \in \mathcal{N}$; all $e=(R, \Omega) \in \mathcal{E}^{N}$, and all $j \in N$,

$$
U_{j}(e):=\left\{\begin{array}{cl}
\min \left\{p\left(R_{j}\right), \lambda\right\} & \text { if } \sum_{N} p\left(R_{i}\right) \geq \Omega \\
\max \left\{p\left(R_{j}\right), \lambda\right\} & \text { if } \sum_{N} p\left(R_{i}\right) \leq \Omega
\end{array}\right.
$$

\footnotetext{
${ }^{3}$ Note that we impose the extra condition of single-valuedness on an allocation rule. Most of the results we present here originally were established for multi-valued allocation rules; see for inistance Ching (1992), Sönmez (1994), and Thomson (1994a,b, 1995a, 1997a). Alternatively, for most results, we could allow for so-called single-plateaued preferences (see for instance Ching 1992) or restrict preferences to the closed interval $[0, \Omega]$.
} 
where $\lambda \in \mathbb{R}_{+}$solves $\sum_{N} U_{i}(e)=\Omega$.

So, in case of excess demand, i.e., $\sum_{N} p\left(R_{i}\right) \geq \Omega$, each agent either receives his optirnal share or a share that is greater than or equal to the share of each other agent. Similarly, in case of excess supply, i.e., $\Sigma_{N} p\left(R_{i}\right) \leq \Omega$, each agent either receives his optimal share or a share that is smaller than or equal to the share of each other agent. ${ }^{6}$ Examples demonstrating the computation of the uniform rulle $U$ are given in Chapter 1, Examples 1.1 and 1.2 .

Another interpretation of this rule is the following "Walrasian interpretation". All agents are asked to maximize their preferences subject to a common upper or lower "budget bound" that is chosen such that feasibility is obtained for this list of maximal shares. ${ }^{7}$

As the following remarks show, the principles underlying the two rules we introduced are also well-known in other models.

Remark 2.1 Bankruptcy and taxation: Consider the situation where a man dies and leaves behind an estate $E$ which is worth less than the sum of his debts. Every claimant $i$ has a claim of size $d_{i}$ on $E$ and the problem is how to divide the total estate $E$ among the claimants. Let $(d, E) \in \mathbb{R}_{+}^{N} \times \mathbb{R}_{+}$, where $E \leq \sum_{N} d_{i}$, denote such a bankruptcy problem. Aumann and Maschler (1985) study, among other "Talmudic solutions", the so-called constraint equal award solution $C E A$. For every bankruptcy problem $(d, E)$ it is defined by $C E A(d, E)=\left(\min \left\{d_{1}, \lambda\right\}, \ldots, \min \left\{d_{n}, \lambda\right\}\right)$, where $\lambda$ is determined by feasibility. In words, this means that all claimants obtain the same award $\lambda$, except that those who claim less than $\lambda$ get their claim. With a slight abuse of notation (replacing the preference profile by a vector of claims) we can state that $C E A(d, E)=U(d, E)$. Dagan and Volij (1993) associate each bankruptcy problem with a bargaining problem and prove that the Nash bargaining solution induces the constrained equal award solution. Since we can associate in a similar way a bargaining problem to each economy with excess demand, for this subclass of economies; the Nash bargaining solution implies the uniform rule (see also Remark 2.2).

Methods for solving bankruptcy problems are formally equivalent to methods of taxation or loss distribution. In a taxation problem, the total tax that has to be paid corresponds to the estate that has to be divided and the taxable income of the individuals correspond to the individual claims. In this setting the proportional rule is applied under the name "flat tax" and the uniform allocation is applied under the name "head tax" (see, for instance, Young 1987, 1988). ${ }^{8}$

Another class of problems that are closely related are cost sharing problems (see for instance Moulin 1987a, 1997). In a cost sharing problem, the total costs of a

\footnotetext{
"Schummer and Thomson (1997) show that the uniform rule assigns the unique Pareto optimal allocation at which the difference between the smallest and the largest share received by any agent is minimal. Furthermore they show that the umiform rule assigns the unique Pareto optimal allocation at which the variance of the agents' shares is minimal.

"I wish to thank William Thomson for bringing this "Wairasian interpretation" of the uniform rule to my attention.

${ }^{8}$ For a survey on bankruptcy ard taxation, see Thomson (1995c).
} 
public facility correspond to the estate that has to be divided and the benefits of the individuals correspond to the individual claims.

Remark 2.2 Bargaining and game theory: Otten, Peters, and Volij (1996) associate with each economy an auxiliary bargaining problem in such a way that its set of Pareto optimal allocations coincides with the set of feasible and Pareto optimal alloca* tions for the economy. They show that the uniform allocation of the economy coincides with both the Nash and lexicographic egalitarian solution outcomes of the associated bargaining problem. Furthermore, they provide two characterizations of the uniform rule, resembling characterizations of the Nash and lexicographic egalitarian solutions.

De Frutos and Massó (1995) show that the uniform rule is the unique rule that selects a Pareto optimal allocation that "Lorenz dominates". all Pareto optimal allocations. Next, they construct for each economy an auxiliary cooperative game with transferable utility and show that the core of this game equals the set of Pareto optimal allocations of the economy. Furthermore, the game is convex. Since for convex games the lexicographic egalitarian solution as introduced by Dutta and Ray (1989) selects the point in the core that is Lorenz maximal, it follows that the uniform allocation coincides with the Dutta-Ray solution outcome.

For more examples of rules we refer the interested reader to a survey of fair allocation when preferences are single-peaked by Thomson (1997b), Chapter 11: "Single-peaked preferences". As a conclusion of this survey Thomson states:

"Our [...] conclusion is that for this model one solution comes out repeatedly as being best-behaved from a variety of viewpoints. The solution is the uniform rule."

In the remainder of this chapter we focus on the uniform rule. In the next section we review most of its desirable properties and characterizations.

\subsection{The uniform rule: properties and characteriza- tions}

One of the most important features of the uniform rule is the fact that it satisfies strategy-proofness, one of the properties first analyzed for (allocation) rules by Sprumont (1991). Strategy-proofness states that no agent ever benefits from misrepresenting his preferences. ${ }^{9}$ Before we give the formal definition, we introduce some notation.

Let $N, M \in \mathcal{N}$ be such that $N \subseteq M$. Let $M \backslash N=\{i \in M \mid i \notin N\}$. For $R \in \mathcal{R}^{M}$ the restriction $\left(R_{i}\right)_{i \in N} \in \mathcal{R}^{N}$ of $R$ to $N$ is denoted by $R_{N}$. Let $R, \tilde{R} \in \mathcal{R}^{N}, i \in N$, and $R_{N \backslash\{i\}}=\bar{R}_{N \backslash\{i\}}$. Then, we call $\bar{R}$ an $i-$ deviation from $R$.

\footnotetext{
In game theoretical terms a rule is strategy-proof if in the direct revelation game form it is a weakly dominant strategy for each agent to announce his true preference relation. Thomson (1990) discusses mamipulability and implementation of allocation rules when preferences are single-peaked.
} 
Strategy-proofness: For all $N \in \mathcal{N}$, all $e=(R, \Omega) \in \mathcal{E}^{N}$, all $\bar{e}=(\tilde{R}, \Omega) \in \mathcal{E}^{N}$, and all $j \in N$, if $\bar{R}$ is a $j$-deviation from $R$, then $\varphi_{j}(e) R_{j} \varphi_{j}(\bar{e})$.

As allready observed by Benassy (1982) in the context of rationing, the uniform rule satisfies strategy-proofriess. On the other hand, it is easy to show that the proportional rule is not strategy-proof.

The next two properties Sprumont (1991) considers are well-known properties. Anonymity requires that the agents' shares do not depend on their names. No-envy states that no agent strictly prefers the share of another agent to his own share.

Let $N \in \mathcal{N}$. A permutation $\pi$ on $N$ is a bijective function $\pi: N \rightarrow N$. By $\Pi^{N}$ we denote the set of all permutations on $N$. Given $\pi \in \Pi^{N}$ and $e=(R, \Omega) \in \mathcal{R}^{N}$, let $R_{\pi}=\left(R_{\pi(i)}\right)_{i \in N}$ and $e_{\pi}=\left(R_{\pi}, \Omega\right)$

Anonymity: For all $N \in \mathcal{N}$, all $e=(R, \Omega) \in \mathcal{E}^{N}$, all $\pi \in \mathbb{I}^{N}$, and all $i \in N$, $\varphi_{i}\left(e_{\pi}\right)=\varphi_{\pi(i)}(e)$

No-envy: For all $N \in \mathcal{N}$, all $e=(R, \Omega) \in \mathcal{E}^{N}$, and all $i, j \in N, \varphi_{i}(e) R_{i} \varphi_{j}(e)$

No-envy can be traced back to Foley (1967) who considers it in the context of resource allocation problems.

The following two characterizations are due to Sprumont (1991).

Theorem 2.1 The uniform rule $U$ is the only rule that satisfies Pareto optimality, strategy-proofness, and anonymity.

Theorem 2.2 The uniform rule $U$ is the only rule that satisfies Pareto optimality, strategy-proofness, and no-envy.

Ching (1992) gives a simple and elegant proof of this last result.

Thomson (1994a) shows that the uniform rule is the only rule that satisfies Pareto optimality, no-envy, and peak-onliness, i.e., the rule only depends on the peaks instead of the complete profile.

Peak-onliness: For all $N \in \mathcal{N}$, all $e=(R, \Omega) \in \mathcal{E}^{N}$, and all $\bar{e}=(\bar{R}, \Omega) \in \mathcal{E}^{N}$, if $p(R)=p(\bar{R})$, then $\varphi(e)=\varphi(\bar{e})$.

Theorem 2.3 The uniform rule $U$ is the only rule that satisfies Pareto optimality, no-envy, and peak-onliness.

The next property we introduce is equal treatment of equals, a weakening of noenvy. It requires that if two agents have the same preference relation, then each of them is indifferent between his share and the other agent's share. 
Equal treatment of equals: For all $N \in \mathcal{N}$, all $e=(R, \Omega) \in \mathcal{E}^{N}$, and all $i, j \in N$, if $R_{i}=R_{j}$, then $\varphi_{i}(e) I_{i} \varphi_{j}(e)$.

If $\varphi$ is in addition Pareto optimal, then equal treatment of equals implies the stronger equality condition $\varphi_{i}(e)=\varphi_{j}(e)$. Note that Pareto optimality and anonymity together imply equal treatment of equals.

The following strengthening of Theorems 2.1 and 2.2 is due to Ching (1994).

Theorem 2.4 The uniform $U$ rule is the only rule that satisfies Pareto optimality, strategy-proofness, and equal treatment of equals.

A summary of the characterizations of the uniform rule by Pareto optimality, strategy-proofness, and either peak-onliness or one of the equity properties anonymity, no-envy, or equal treatment of equals, can be found in Table 2.1 .

\begin{tabular}{|l||c|c|c|c|}
\hline & Th & Th & Th & Th \\
& 2.1 & 2.2 & 2.3 & 2.4 \\
\hline \hline Pareto optimality & $\times$ & $\times$ & $\times$ & $\times$ \\
\hline strategy-proofness & $\times$ & $\times$ & & $\times$ \\
\hline anonymity & $\times$ & & & \\
\hline no-envy & & $\times$ & $\times$ & \\
\hline peak-onliness & & & $\times$ & \\
\hline equal treatment of equals & & & & $\times$ \\
\hline
\end{tabular}

Table 2.1: Characterizations of the uniform rule by Pareto optimality, strategyproofness, peak onliness, and equity properties.

Next, we discuss so-called "solidarity properties" that describe the effect of certain changes in a single parameter of the economy while the other parameters are kept fixed, e.g., the endowment, the population, or the preferences. If after such a change either all agents that are not responsible for the change (weakly) lose together or all (weakly) gain together, then, depending on which parameter has been changed, the rule satisfles resource-monotonicity, population-monotonicity ${ }^{10}$, or welfare-domination under preference-replacement $t^{11}$. Thomson $(1994 \mathrm{a}, 1995 \mathrm{a}, 1997 \mathrm{a})$ shows that these properties are generally incompatible with the properties we introduced above. However, he shows that these incompatibilities only occur when the change in the parameter is such that it turns an economy in which there is "too much" to divide into an economy in which there is "too little" to divide, or vice versa. We call a change where this does not

\footnotetext{
${ }^{10}$ Thomson (1983) introduced population-monotonicity in the context of bargaining. For a surwey on population-monotonicity, see Thomson (1995b).

${ }^{11}$ Moulin (1987b) introduced this "replacement principle" in the context of binary choice with quasilinear preferences. He calls it "agreement". Thomson $(1993,1996 a, 1997 a)$ studies the replacement principle in different settings, e.g., in public and private good economies with single-peaked preferences.
} 
occur one-sided: let $N, \bar{N} \in \mathcal{N}, e=(R, \Omega) \in \mathcal{E}^{N}$, and $\bar{e}=(\bar{R}, \bar{\Omega}) \in \mathcal{E}^{\bar{N}}$. If either $\left[\sum_{N} p\left(R_{i}\right) \geq \Omega\right.$ and $\left.\sum_{\tilde{N}} p\left(\bar{R}_{i}\right) \geq \bar{\Omega}\right]$ or $\left[\sum_{N} p\left(R_{i}\right) \leq \Omega\right.$ and $\left.\sum_{\bar{N}} p\left(\bar{R}_{i}\right) \leq \bar{\Omega}\right]$, then $\bar{e}$ is a one-sided change of $e$.

Thomson shows that a wide class of rules satisfies the "one-sided" versions of the solidarity properties, i.e, solidarity is only required for one-sided changes. However, the uniform rule turns out to be essentially the only rule that satisfies any of the one-sided solidarity properties, together with Pareto optimality and no-envy.

First, we consider a one-sided change in the endowment, keeping the set of agents and their preferences fixed (Thomson 1994a). One-sided resource-monotonicity states that after such a change, either all agents (weakly) lose or all (weakly) gain.

One-sided resource-monotonicity: For all $N \in \mathcal{N}$, all $e=(R, \Omega)$, and all $\bar{e}=(R, \bar{\Omega}) \in \mathcal{E}^{N}$, if $\bar{e}$ is a one-sided change of $e$, then either [for all $\left.i \in N, \varphi_{i}(e) R_{i} \varphi_{i}(\bar{e})\right]$ or [for all $\left.i \in N, \varphi_{i}(\bar{e}) R_{i} \varphi_{i}(e)\right]$.

Next, we consider the arrival of new agents so that this change in the set of agents is one-sided, keeping the preferences of the remaining agents and the endowment fixed (Thomson 1995a). One-sided population-monotonicity states that either all agents initially present (weakly) lose or all (weakly) gain.

One-sided population-monotonicity: For all $N, \bar{N} \in \mathcal{N}$, all $e=(R, \Omega) \in \mathcal{E}^{N}$, and all $\bar{e}=(\bar{R}, \Omega) \in \mathcal{E}^{\bar{N}}$, if $N \subseteq \bar{N}, R=\bar{R}_{N}$, and $\bar{e}$ is a one-sided change of $e$, then either [for all $i \in N, \varphi_{i}(e) R_{i} \varphi_{i}(\bar{e})$ ] or [for all $i \in N, \varphi_{i}(\bar{e}) R_{i} \varphi_{i}(e)$ ].

Finally, we consider a one-sided change of one agent's preference relation, keeping the preferences of the remaining agents, the set of agents, and the endowment fixed (Thomson 1997a). One-sided welfare-domination under preference-replacement states that either all remaining agents (weakly) lose or all (weakly) gain.

One-sided welfare-domination under preference-replacement, or one-sided replacement-domination: For all $N \in \mathcal{N}$, all $e=(R, \Omega) \in \mathcal{E}^{N}$, all $\bar{e}=(\bar{R}, \Omega) \in \mathcal{E}^{N}$, and all $j \in N$, if $\bar{R}$ is a $j$-deviation from $R$ and $\bar{e}$ is a one-sided change of $\dot{e}$, then either [for all $\left.i \in N \backslash\{j\}, \varphi_{i}(e) R_{i} \varphi_{i}(\bar{e})\right]$ or [for all $\left.i \in N \backslash\{j\}, \varphi_{i}(\bar{e}) R_{i} \varphi_{i}(e)\right]$.

In the sequel we use the shorter phrase of one-sided replacement-domination for one-sided welfare-domination under preference-replacement.

On the domain of economies with bounded preferences, $\cup_{N \in \mathcal{N}} \mathcal{E}_{b}^{N}$, Thomson (1994a) establishes the following characterization of the uniform rule.

Theorem 2.5 On the domain of economies with bounded preferences the uniform rule $U$ is the only rule that satisfies Pareto optimality, no-envy, and one-sided resourcemonotonicity. 
Similar characterizations of the uniform rule by Pareto optimality, no-envy, and one of the remaining solidarity conditions can be derived if the following well-known condition of replication-invariance is added (Thomson 1994b, 1995a, 1997a). Replicationinvariance states that if an economy is replicated, i.e., the endowment and the preference profile are replicated, then also the allocation assigned by the rule must be replicated. In order to formally describe a replicated economy, we introduce the following notation.

Let $k \in \mathbb{N}$ and $\bar{N}, N_{1}, \ldots, N_{k} \in \mathcal{N}$ such that for all $l \in\{1, \ldots, k\}, N_{l} \neq 0$. Then, the collection $\left\{N_{1}, \ldots, N_{k}\right\}$ constituties a partition of $\bar{N}$ if $\bar{N}=\bigcup_{l \in\left\{1_{1}, \ldots k\right\}} N_{l}$ and $N_{l} \cap N_{m}=\emptyset$ for all $l, m \in\{1, \ldots k\}, l \neq m$.

Let $N, \bar{N} \in \mathcal{N}, e=(R, \Omega) \in \mathcal{E}^{N}$, and $\bar{e}=(\bar{R}, \bar{\Omega}) \in \mathcal{E}^{N}$ be such that

(i) there exist $k \in \mathbb{N}$ and $N_{1}, \ldots, N_{k} \in \mathcal{N}$ such that $N=N_{1}$ and $\left\{N_{1}, \ldots, N_{k}\right\}$ constitutes a partition of $\vec{N}$,

(ii) there exist $k$ bijective functions, indexed by $l \in\{1, \ldots, k\}, b_{l}: N \rightarrow N_{l}$, such that for all $i \in N, R_{i}=\bar{R}_{b_{t}(i)}{ }^{12}$

(iii) $\bar{\Omega}=k \Omega$.

Then, $\bar{e}$ is a $k$-replica of $e$. Let $\bar{e} \in \mathcal{E}^{N}$ be a $k$-replica of $e \in \mathcal{E}^{N}$ and let $x \in \mathbb{R}_{+}^{N}$. If $\bar{x} \in \mathbb{R}_{+}^{\bar{N}}$ is such that for all $i \in N, x_{i}=\bar{x}_{b_{l}(i)}$ for the functions $b_{l}, l \in\{1, \ldots k\}$, as described in (ii), then $\bar{x}$ a k-replica of $x$.

Replication-invariance: For all $N, \bar{N} \in \mathcal{N}$, all $e=(R, \Omega) \in \mathcal{E}^{N}$, and all $\bar{e}=$ $(\bar{R}, \bar{\Omega}) \in \mathcal{E}^{\bar{N}}$, if $\bar{e}$ is a $k$-replica of $e_{\text {, then }} \varphi(\bar{e})$ is a $k$-replica of $\varphi(e)$.

Note that if a rule is replication-invariant, then is satisfies anonymity.

On the domain of economies with bounded preferences, $\bigcup_{N \in \mathcal{N}} \mathcal{E}_{b}^{N}$, Thomson (1995a) establishes the following characterization of the uniform rule.

Theorem 2.6 On the domain of economies with bounded preferences, the unifom rule $U$ is the only rule that satisfies Pareto optimality, no-envy, one-sided populationmonotonicity, and replication-invariance.

Question 1: Following the proof of this Theorem, Thomson asks the question whether replication-invariance is independent from the other characterizing properties (see Thom son 1995 a, page 242 ). ${ }^{13}$

\footnotetext{
${ }^{12}$ Let $l \in\{\mathbb{1}, \ldots k\}$ and $i \in N$. Then, agent $b_{l}(i)$ is the "replica", or the "clone", of agent $i \in N$ in $N_{i}$.

${ }^{13}$ Thomson also asks the question whether the wiform rule is the only rule that satisfies Pareto optimality, individual rationality from equal division, one-sided population-monotonicity, and replicationinvariance.

The answer is that there is a large class of rules satisfying the properties mentioned in the question. Two examples are the "proportional reallocation rule" $\mathrm{Pr}^{\mathrm{r}}$ operated from equal division (see Section 4.2) and the "maximally satiating reallocation rule" $\varphi^{\max }$ operated from equal division (see Section 4.6 ).
} 


\begin{tabular}{|l|c|c|c|}
\hline & Th & Th & Th \\
\hline Pareto optimality & 2.5 & 2.6 & 2.7 \\
\hline no-envy & $\times$ & $\times$ & $\times$ \\
\hline o-s resource-monotonicity & $\times$ & & \\
\hline o-s popwlation-monotonicity & & $\times$ & \\
\hline o-s replacement-domination & & & $\times$ \\
\hline replicailion-invariance & & $\times$ & $\times$ \\
\hline
\end{tabular}

Table 2.2: Characterizations of the uniform rule by Pareto optimality, no-nvy, and solidarity properties.

We answer this question in the next section.

The next characterization of the uniform rule is due to Thomson (1997a).

Theorem 2.7 The uniform rule $U$ is the only rule that satisfies Pareto optimality, no-envy, onc-sided replacement-domination, and replication-invariance:

Question 2: Following the proof of this Theorem, Thomson asks the question whether replication-invariance is independent from the other characterizing properties (see Thomson $1997 a$, page 161).

We answer this question in the next section.

A summary of the characterizations of the uniform rule by Pareto optimality, noenvy, and one of the solidarity properties one-sided resource-monotonicity, one-sided population-monotonicity, or one-sided replacement-domination, can be found in Table 2.2 .

The next property we introduce is consistency. Essentially it describes the stability of a recommended allocation in situations where agents leave with their shares: let $N \in \mathcal{N}$ be a group of agents, $e=(R, \Omega) \in \mathcal{E}^{N}$ an economy for $N$, and $\pi \in \mathbb{R}_{+}^{N}$ the allocation assigned by the rule. Let $M \subseteq N$ and suppose all agents in $N \backslash M$ leave with the sheres assigned to them at $x$. By doing so they create the reduced economy $\left(R_{M}, \Sigma_{M} x_{1}\right) \in \mathcal{E}^{M}$. Now, by consistency, applying the rule to the reduced economy yields the same shares for the remaining agents in $M$ as in the original economy. ${ }^{14}$

Let $M, N \in \mathcal{N}$ such that $M \subseteq N$. For $x \in \mathbb{R}^{N}$, the restriction $\left(x_{i}\right)_{i \in M} \in \mathbb{R}^{M}$ of $x$ to $M$ is denoted by $x_{M}$.

Consistency: For all $M, N \in \mathcal{N}$ with $M \subseteq N$ and all $\varepsilon=(R, \Omega) \in \mathcal{E}^{N}, \varphi(e)_{M}=$ $\varphi\left(R_{M}, \sum_{M} \varphi_{i}\left(e_{j}^{j}\right)\right.$.

\footnotetext{
${ }^{14}$ For a survey on consistency, see Thomson (1996b).
} 
A weakening of consistency is bilateral consistency which is obtained from the former condition by limiting its application to subgroups of remaining agents of size two.

Bilateral consistency: For all $M, N \in \mathcal{N}$ with $M \subseteq N$ and $|M|=2$ and all $e=(R, \Omega) \in \mathcal{E}^{N}, \varphi(e)_{M}=\varphi\left(R_{M}, \sum_{M} \varphi_{i}(e)\right)$.

The next characterization of the uniform rule contains the following notion of "endowment continuity".

$\Omega$-continuity: For all $N \in \mathcal{N}$, all $e=(R, \Omega) \in \mathcal{E}^{N}$, and all sequences $\left\{\Omega^{k}\right\}_{k \in \mathbb{N}}$ in $\mathbb{R}_{+}$, if $\lim _{k \rightarrow \infty} \Omega^{k}=\Omega$, then $\lim _{k \rightarrow \infty} \varphi\left(R, \Omega^{k}\right)=\varphi(R, \Omega)$.

The next characterization of the uniform rule is due to Thomson (1994b).

Theorem 2.8 The uniform rule $U$ is the only rule that satisfies Pareto optimality, no-envy, bilateral consistency, and $\Omega$-continuity.

Next, Thomson (1994b) shows that no-envy can be replaced by the following "minimal right" condition: each agent (weakly) prefers his share to the "equal division share" of the economy.

Individual rationality from equal division: For all $N \in \mathcal{N}$, all $e=(R, \Omega) \in \mathcal{E}^{N}$, and all $i \in N, \varphi_{i}(e) R_{i} \frac{\Omega}{|N|}$.

Theorem 2.9 The uniform rule $U$ is the only rule that satisfies Pareto optimality, individual rationality from equal division, bilateral consistency, and $\Omega$-continuity.

Furthermore, Thomson (1994b) shows that in Theorem 2.9, $\Omega$-continuity can be replaced by replication-invariance.

Theorem 2.10 The uniform rule $U$ is the only rule that satisfies Pareto optimality, individual rationality from equal division, consistency, and replication-invariance.

Dagan (1996) presents the following results that can be seen as variants of Theorems 2.8 and 2.9. In contrast with Thomson (1994b), Dagan only considers single-valued rules. Under this "extra assumption" he shows that even if the set of potential agents is bounded from above, but contains at least four agents, the uniqueness of the uniform rule as established in Theorems $2.8,2.9$, and 2.10 is achieved without assuming $\Omega$ continuity, or replication-invariance respectively.

Theorem 2.11 Let the set of potential agents contain at least four agents. Then, the uniform rule $U$ is the only rule that satisfies Pareto optimality, no-envy, and bilateral consistency. 


\begin{tabular}{|l|c|c|c|c|c|c|}
\hline & Th & Th & Th & Th & Th & Th \\
\hline Pareto optimality & 2.8 & 2.9 & 2.10 & 2.11 & 2.12 & 2.13 \\
\hline consistency & $\times$ & $\times$ & $\times$ & $\times$ & $\times$ & $\times$ \\
\hline bilateral consistency & $\times$ & $\times$ & & $\times$ & $\times$ & \\
\hline converse consistency & & & & & & $\times$ \\
\hline $\begin{array}{l}\text { individual rationality } \\
\text { from equal division }\end{array}$ & & $\times$ & $\times$ & & $\times$ & $\times$ \\
\hline no-nvy & $\times$ & & & $\times$ & & \\
\hline amonymity & & & & & & $\times$ \\
\hline R-continuity & $\times$ & & & & & \\
\hline replication-invariance & & $\times$ & $\times$ & & & \\
\hline
\end{tabular}

Table 2.3: Characterizations of the uniform rule by Pareto optimality, consistency properties, etc.

Theorem 2.12 Let the set of potential agents contain at least four agents. Then, the uniform rule $U$ is the only rule that satisfies Pareto optimality, individual rationality from equal division, and bilateral consistency.

The last property we introduce is converse consistency, a property that determines the desirability of an allocation for an economy on the basis of the desirability of its restrictions to all two-agent reduced economies. So, given a feasible allocation $x$ for an economy, if for every associated two-agent reduced economy the restriction of $x$ to these two agents equals the allocation chosen by the rule for this two-agent reduced economy, then $x$ must be the allocation assigned by the rule. ${ }^{15}$

Converse consistency: For all $N \in \mathcal{N}$, all $e=(R, \Omega) \in \mathcal{E}^{N}$, and all feasible allocations $x \in \mathbb{R}_{++}^{N}$, if for all $M \subseteq N$ with $|M|=2, \varphi\left(R_{M}, \sum M x_{i}\right)=x_{M}$, then $\varphi(e)=x$.

The next characterization of the uniform rule is due to Thomson (1994b).

Theorem 2.13 The uniform rule $U$ is the only rule that satisfies Pareto optimality, individual rationality from equal division, converse consistency, and anonymity.

A summary of the characterizations of the uniform rule by Pareto optimality, individual rationality from equal division, no-envy, and one of the consistency properties consistency, bilateral consistency, or converse consistency, can be found in Table 2.3 .

The following characterizations of the uniform rule, due to Sönmez (1994), do not rely on Pareto optinality, a condition that until now was always included.

Theorem 2.14 The uniform rule $U$ is the only rule that satisfies individual rationality from equal division, one-sided resource-monotonicity, and consistency.

\footnotetext{
15For a survey on converse consistency, see Thomson (1996b).
} 


\begin{tabular}{|l||c|c|c|}
\hline & Th & Th & Th \\
& 2.14 & 2.15 & 2.16 \\
\hline \hline individual rationality from equal division & $\times$ & $\times$ & $\times$ \\
\hline o-s resource-monotonicity & $\times$ & & $\times$ \\
\hline o-s population-monotonicity & & $\times$ & \\
\hline consistency & $\times$ & $\times$ & \\
\hline converse consistency & & & $\times$ \\
\hline replication-invariance & & $\times$ & \\
\hline
\end{tabular}

Table 2.4: Characterizations of the uniform rule without Pareto optimality.

Theorem 2.15 The uniform rule $U$ is the only rule that satisfies individual rationality from equal division, one-sided population-monotonicity, consistency, and replicationinvariance.

Theorem 2.16 The uniform rule $U$ is the only rule that satisfies individual rationality from equal division, one-sided resource-monotonicity, and converse consistency.

A summary of these last characterizations of the uniform rule without Pareto optimality can be found in Table 2.4 .

Finally, even though the list of characterizations of the uniform rule we reviewed in this section is impressive (see Tables 2.1-2.4), we cannot claim completeness. For further properties and characterizations of the uniform rule that are related to bargaining theory and cooperative game theory we refer the interested reader to the references given in Remark 2.2.

\subsection{The independence of replication-invariance}

In this section we answer the open questions whether in the characterizations of the uniform rule stated in Theorems 2.6 and 2.7 replication-invariance is independent from the remaining properties (see Section 2.2, Questions 1 and 2). We do so by constructing a rule that satisfies Pareto optimality, no-envy, one-sided population-monotonicity, onesided replacement-domination, but not replication-invariance.

Before we define the "absorbing agent" rule $\varphi^{a}$, we introduce some notation. ${ }^{16}$ Let $N \in \mathcal{N}$. Then, by $\mathcal{A}^{N} \subseteq \mathcal{E}^{N}$ we denote the following subclass of economies: for $(R, \Omega) \in \mathcal{A}^{N}$ we have that (i) $\sum_{N} p\left(R_{i}\right)<\Omega$ and (ii) there exists an agent $j \in N$ such that $p\left(R_{j}\right)>\frac{\Omega}{2}$.

Hence, for $N \in \mathcal{N}$ and $e=(R, \Omega) \in \mathcal{A}^{N}$ there is (i) too much to distribute and (ii) there is exactly one agent, namely agent $j$, with a relatively large peak (see Figure 2.1). For such an economy, we construct the allocation $\varphi^{a}(e)$ as follows.

\footnotetext{
16 The $a$ of $\varphi^{a}$ stands for "answer", or alternatively for "absorbing agent".
} 


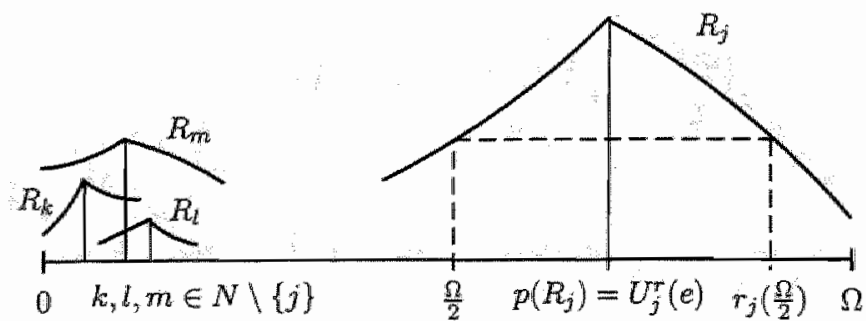

Figure 2.1: An econamy $e \in \mathcal{A}^{N}$.

We start from the uniform allocation $U(e)$ and let agent $j$ "absorb" some part of the excess supply the remaining agents, $i \in N \backslash\{j\}$, experience at their uniform shares, denoted by the list $\left(U_{i}(e)\right)_{i \in N \backslash\{j\}}$.

We denote the excess supply of the agents in $N \backslash\{j\}$ at the allocation $U(e)$ by $s_{j}(e)=$ $\sum_{N \backslash\{j\}}\left(U_{i}(e)-p\left(R_{i}\right)\right)$.

Note that in order to preserve same-sidedness, we let agent $j$ absorb at most $s_{j}(e)$. However , when we let agent $j$ absorb part of, or even the whole excess supply $s_{j}(e)$, we do not want him to envy the other agents. Therefore, we introduce the sufficiently small upper bound $r_{j}\left(\frac{\Omega}{2}\right)$ for agent $j$ 's share. Recall that either $r_{j}\left(\frac{\Omega}{2}\right) I_{j} \frac{\Omega}{2}$ or $r_{j}\left(\frac{\Omega}{2}\right)=\infty$. Note that the shares of all other agents are smaller than $\frac{\Omega}{2}$ and therefore, as long as agent $j$ 's share is in between his peak $p\left(R_{j}\right)$ and $r_{j}\left(\frac{\Omega}{2}\right)$, he does not envy the other agents.

Summarizing, if $N \in \mathcal{N}$ and $e=(R, \Omega) \in \mathcal{A}^{N}$, then we obtain $\varphi^{a}(e)$ from $U(e)$ by letting agent $j$ absorb the amount $a(e):=\min \left\{s_{j}(e), r_{j}\left(\frac{\Omega}{2}\right)-p\left(R_{j}\right)\right\}$ and subtracting this amount uniformly from the uniform shares of the other agents (see Figures 2.2 and 2.3). If $N \in \mathcal{N}$ and $e=(R, \Omega) \notin \mathcal{A}^{N}$, then we apply the uniform rule.

The rule $\varphi^{a}$ : For all $N \in \mathcal{N}$ and all $e=(R, \Omega) \in \mathcal{A}^{N}$, let $j \in N$ be such that $p\left(R_{j}\right)>\frac{n}{2}$. Then, for all $i \in N$,

$$
\varphi_{i}^{a}(e)= \begin{cases}p\left(R_{j}\right)+a(e) & \text { if } i=j \\ U_{i}\left(R_{N \backslash j\}}, \Omega-\varphi_{j}^{a}(\epsilon)\right) & \text { if } i \in N \backslash\{j\} .\end{cases}
$$

For all $N \in \mathcal{N}$ and all $e=(R, \Omega) \notin A^{N}$,

$$
\varphi^{a}(e)=U(e) .
$$

The rule $\varphi^{a}$ is by construction same-sided and therefore Pareto optimal. Since we either assign the uniform allocation to an economy or change the uniform allocation in such a way that envy between the agents cannot arise, $\varphi^{a}$ satisfies no-envy. Furthermore, it is easy to check that the rule $\varphi^{a}$ satisfes other well-known properties for division rules, such as anonymity, continuity with respect to the preferences (Sprumont 1991), $\Omega$-continuity, and individual rationality from equal division. Given this list of properties 


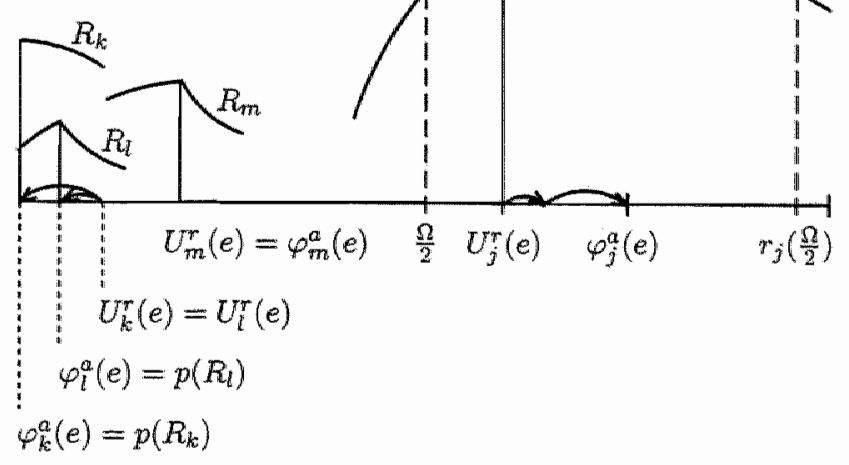

Figure 2.2: The rule $\varphi^{a}$ for an economy $e \in \mathcal{A}^{N}$ with $a(e)=s_{j}(e)$.

and comparing it to the list of properties given in the characterizations of the uniform rule we recalled in Section 2.2 (e.g., Theorems $2.1,2.3,2.5,2.9$, and 2.13) it follows immediately that $\varphi^{a}$ satisfies none of the properties strategy-proofness, peak-onliness, one-sided resource-monotonicity, bilateral consistency, and converse consistency.

The following example demonstrates that the rule $\varphi^{a}$ does not satisfy replicationinvariance.

Example 2.1 Let $N=\{1,2\}$ and $R \in \mathcal{R}^{N}$ be such that $p(R)=(1,4)$, and $r_{2}(3)=5$. Let $\Omega=6$ and consider the economy $e=(R, \Omega)$. Thus, $p\left(R_{1}\right)+p\left(R_{2}\right)<\Omega$ and $p\left(R_{2}\right)>\frac{\Omega}{2}$. Hence, $(R, \Omega) \in \mathcal{A}^{N}$ and $U(e)=(2,4), \varphi^{a}(e)=(1,5)$.

Let $N_{1}=N$ and $N_{2}=\{3,4\}$ be such that $R_{3}=R_{1}$ and $R_{4}=R_{2}$. Let $\bar{N}=N_{1} \cup N_{2}$, $\tilde{R}=\left(R_{1}, R_{2}, R_{3}, R_{4}\right)$, and $\bar{\Omega}=12$. Hence, $(\bar{R}, \bar{\Omega}) \in \mathcal{E}^{\bar{N}}$ is a 2-replica of $(R, \Omega)$. Since there exists no $i \in \bar{N}$ such that $p\left(R_{i}\right)>\frac{\Omega}{2}=6$, it follows that $\bar{e}=(\bar{R}, \bar{\Omega}) \notin \mathcal{A}^{N}$ and $U(\bar{e})=(2,4,2,4)=\varphi^{a}(\bar{e})$. Thus, $\varphi^{a}(\bar{e})$ is not a 2-replica of $\varphi^{a}(\bar{e})$. Hence, $\varphi^{a}$ is not replication-invariant.

Remark 2.3 Note that for all $N \in \mathcal{N}$, all $e=(R, \Omega) \in \mathcal{A}^{N}$, all $k \in \mathbb{N}$, and all $k$ replica $\bar{e}=(\bar{R}, \bar{\Omega}) \in \mathcal{E}^{\bar{N}}$ of $e$ it follows that $\ddot{e} \notin \mathcal{A}^{\bar{N}}$ and that $\varphi^{\alpha}(\bar{e})$ is not a $k$-replica of $\varphi^{\alpha}(e)$.

Lemma 2.1 The rule $\varphi^{a}$ satisfies one-sided population-monotonicity.

Proof. Let $N, \bar{N} \in \mathcal{N}, e=(R, \Omega) \in \mathcal{E}^{N}$, and $\bar{e}=(\bar{R}, \Omega) \in \mathcal{E}^{\bar{N}}$ be such that $N \subseteq \bar{N}$ and $R=\vec{R}_{N}$. Now, it is sufficient to show that, if $\bar{e}$ is a one-sided change of $e$, then either (i) [for all $\left.i \in N, \varphi_{i}^{a}(e) R_{i} \varphi_{i}^{a}(\bar{e})\right]$ or (ii) [for all $\left.i \in N, \varphi_{i}^{a}(\bar{e}) R_{i} \varphi_{i}^{a}(e)\right]$. Let $\ddot{e}$ be a one-sided change of $e$. 


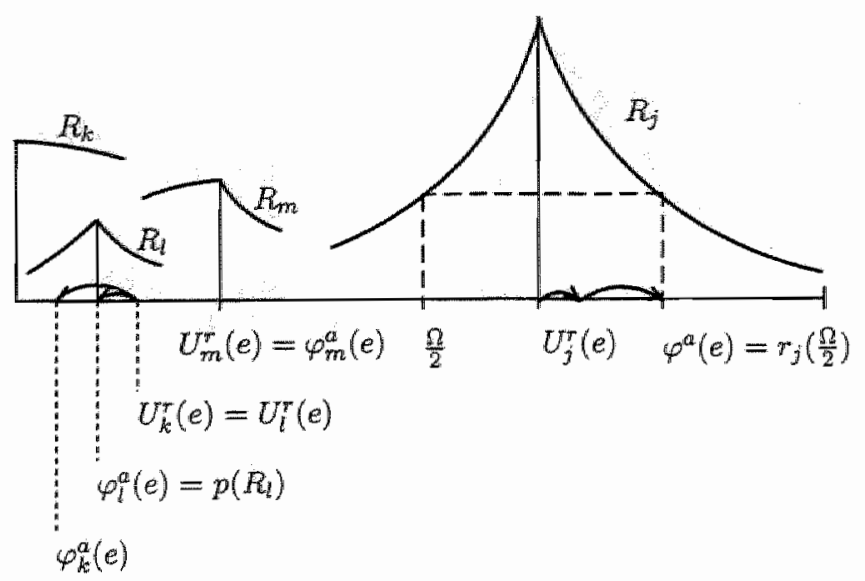

Figure 2.3: The rule $\varphi^{a}$ for an economy $e \in A^{N}$ with $a(e)=r_{j}\left(\frac{\Omega}{2}\right)-p\left(R_{j}\right)$.

Case 1: $\quad e \notin \mathcal{A}^{N}, \bar{e} \notin \mathcal{A}^{\bar{N}}$. Then, $\varphi^{a}(e)=U(e)$ and $\varphi^{a}(\bar{e})=U(\bar{e})$. Since, the uniform rule is one-sided population-monotonic, either (i) or (ii) holds.

Case 2: $e \in \mathcal{A}^{N}, \bar{e} \notin \mathcal{A}^{\bar{N}}$. Hence, $\sum_{N} p\left(R_{i}\right)<\Omega$ and there exists $j \in N$ such that $p\left(R_{j}\right)>\frac{\Omega}{2}$. Then, $\sum_{\bar{N}} p\left(\bar{R}_{i}\right) \leq \Omega$. Since, $\bar{\varepsilon} \notin \mathcal{A}^{\bar{N}}$, we have $\sum_{\bar{N}} p\left(\bar{R}_{i}\right)=\Omega$. So, by Pareto optimality, for all $i \in N, \varphi_{i}^{a}(\bar{e})=p\left(R_{i}\right)$. This implies (ii).

Case 3: e $\notin \mathcal{A}^{N}, \bar{e} \in \mathcal{A}^{\bar{N}}$. Hence, $\sum_{N} p\left(R_{i}\right) \leq \sum_{N} p\left(\bar{R}_{i}\right)<\Omega$ and there exists $j \in \bar{N} \backslash N$ such that $p\left(\bar{R}_{j}\right)>\frac{\Omega}{2}$. Let $R^{\prime}=\bar{R}_{\bar{N} \backslash(j\}} \in \bar{R}^{\bar{N}\{j\}}$ "Then, for all $i \in N$,

$$
\varphi_{i}^{a}(e)=U_{i}(e) \text { and } \varphi_{i}^{a}(\bar{e})=U_{i}\left(R^{\prime}, \Omega-\varphi_{j}^{a}(\bar{e})\right) \text {. }
$$

Since the uniform rule is one-sided resource-monotonic and $\left(R^{\prime}, \Omega\right),\left(R^{\prime}, \Omega-\varphi_{j}^{a}(\bar{e})\right) \in$ $\varepsilon^{N \backslash j\}}$ are such that $\sum_{N \backslash, j\}} p\left(R_{i}^{\prime}\right) \leq \Omega-\varphi_{j}^{a}(\bar{e})<\Omega$, it follows that for all $i \in N \subseteq$ $N\{\{j\}$.

$$
U_{i}\left(R^{\prime}, \Omega-\varphi_{j}^{a}(\bar{e})\right) R_{i} U_{i}\left(R^{\prime}, \Omega\right)
$$

Since the uniform rule is one-sided population-monotonic and $e=(R, \Omega) \in \mathcal{E}^{N}$, $\left(R^{\prime}, \Omega\right) \in \mathcal{E}^{\tilde{N} \backslash(j)}$ are such that $N \subseteq N \backslash\{j\}, R=R_{N \text {, and }} \sum_{N \backslash, j\}} p\left(R_{i}^{\prime}\right) \leq \Omega$, it follows that for all $i \in N$.

$$
U_{i}(R, \Omega) R_{i} U_{i}(e)
$$

Thus, (2.1), (2.2), and (2.3) together imply that for all $i \in N, \varphi_{i}^{a}(\bar{e}) R_{i} \varphi_{i}^{a}(e)$. Hence, (ii) holds.

Case 4: e $\in \mathcal{A}^{N}, \bar{e} \in \mathcal{A}^{\bar{N}}$. Hence, $\sum_{N} p\left(R_{i}\right) \leq \sum_{N} p\left(\bar{R}_{i}\right)<\Omega$ and there exists $j \in N \subseteq \bar{N}$ such that $p\left(R_{j}\right)>\frac{\Omega}{2}$. Let $R^{\prime}=R_{N \backslash j\}} \in \mathcal{R}^{N \backslash j\}}$ and $\bar{R}^{\prime}=\bar{R}_{\bar{N} \backslash\{j\}} \in \mathcal{R}^{\bar{N} \backslash\{j\}}$. Then,

$$
\varphi_{i}^{a}(e)= \begin{cases}p\left(R_{j}\right)+a(e) & \text { if } i=j \\ U_{i}\left(R^{\prime}, \Omega-\varphi_{j}^{a}(e)\right) & \text { if } i \in N \backslash\{j\}\end{cases}
$$




$$
\varphi_{i}^{a}(\bar{e})= \begin{cases}p\left(R_{j}\right)+a(\bar{e}) & \text { if } i=j \\ U_{i}\left(\bar{R}^{\prime}, \Omega-\varphi_{j}^{a}(\bar{e})\right) & \text { if } i \in \bar{N} \backslash\{j\} .\end{cases}
$$

Note that $U_{j}(e)=p\left(R_{j}\right)=U_{j}(\bar{e})$. Therefore, $\Omega-U_{j}(e)=\sum_{N \backslash\{j\}} U_{i}(e)=\sum_{\hat{N} \backslash\{j\}} U_{i}(\bar{e})$ and $s_{j}(e) \geq s_{j}(\bar{e})$. Hence, $a(e) \geq a(\bar{e})$ and $p\left(R_{j}\right) \leq \varphi_{j}^{\alpha}(\bar{e}) \leq \varphi_{j}^{a}(e)$. Thus,

$$
\varphi_{j}^{\alpha}(\bar{e}) R_{j} \varphi_{j}^{\alpha}(e)
$$

Now, we have either

(a) $a(\bar{e})=s_{j}(\bar{e})$ or

(b) $a(\bar{e})=r_{j}\left(\frac{\Omega}{2}\right)-p\left(R_{j}\right)$.

Suppose (a) holds. Then, for all $i \in N \backslash\{j\}, \varphi_{i}^{a}(\bar{e})=p\left(R_{i}\right)$. Thus, for all $i \in M \backslash\{j\}$,

$$
\varphi_{i}^{a}(\bar{e}) R_{i} \varphi_{i}^{a}(e) .
$$

Hence, (2.5) and (2.6) imply (ii).

Suppose (b) holds. Then, $a(\bar{e})=a(e)$ and $\Omega-\varphi_{j}^{\alpha}(\bar{e})=\Omega-\varphi_{j}^{a}(e)$. Since the uniform rule is one-sided population-monotonic and $\left(R^{\prime}, \Omega-\varphi_{j}^{a}(e)\right) \in \mathcal{E}^{M \backslash j j\}},\left(\bar{R}^{\prime}, \Omega-\varphi_{j}^{a}(\bar{e})\right) \in$ $\mathcal{E}^{\bar{N} \backslash\{j\}}$ are such that $N \backslash\{j\} \subseteq \bar{N} \backslash\{j\}, R^{\prime}=\bar{R}_{N \backslash\{j\}}^{\prime}$, and $\sum_{\bar{N} \backslash\{j\}} p\left(\vec{R}_{i}\right) \leq \Omega-\varphi_{j}^{a}(e)$, it follows that for all $i \in N \backslash\{j\}$,

$$
U_{i}\left(\bar{R}^{\prime}, \Omega-\varphi_{j}^{a}(\bar{e})\right) R_{i} U_{i}\left(R^{\prime}, \Omega-\varphi_{j}^{a}(e)\right) .
$$

Thus, by (2.4), for all $i \in N \backslash\{j\}$,

$$
\varphi_{i}^{a}(\bar{e}) R_{i} \varphi_{i}^{a}(e)
$$

Hence, (2.5) and (2.8) imply (ii).

Lemma 2.2 The rule $\varphi^{a}$ satisfies one-sided replacement-domination.

Proof. Let $N \in \mathcal{N}, e=(R, \Omega), \bar{e}=(\bar{R}, \Omega) \in \mathcal{E}^{N}$, and $k \in N$ be such that $\ddot{R}$ is a $k$-deviation from $R$. It is sufficient to show that if $\bar{e}$ is a one-sided change of $e$, then either (i) [for all $\left.i \in N \backslash\{k\}, \varphi_{i}(e) R_{i} \varphi_{i}(\tilde{e})\right]$ or (ii) [for all $\left.i \in N \backslash\{k\}, \varphi_{i}^{a}(\bar{e}) R_{i} \varphi_{i}^{a}(e)\right]$. Let $e$ be a one-sided change of $e$.

Case 1: $e \notin \mathcal{A}^{N}, \bar{e} \notin \mathcal{A}^{N}$. Then, $\varphi^{a}(e)=U(e)$ and $\varphi^{\alpha}(\bar{e})=U(\bar{e})$. Since, the uniform rule satisfies one-sided replacement-domination, either (i) or (ii) holds.

Case 2: $\quad e \notin \mathcal{A}^{N}, \bar{e} \in \mathcal{A}^{N}$. Hence, $\bar{R}$ and $R$ are such that $p\left(R_{k}\right) \leq \frac{\Omega}{2}, p\left(\tilde{R}_{k}\right)>\frac{\Omega}{2}$, and $\sum_{N} p\left(R_{i}\right) \leq \sum_{N} p\left(\bar{R}_{i}\right)<\Omega$. Thus, $U_{k}(e) \leq \frac{\Omega}{2}<p\left(\bar{R}_{k}\right) \leq \varphi_{k}^{a}(\bar{R}, \Omega)$. Let $R^{\prime}=R_{N \backslash\{k\}} \in$ $\mathcal{R}^{N \backslash\{k\}}$. Then, for all $i \in N \backslash\{k\}$,

$$
\varphi_{i}^{a}(e)=U_{i}(e)=U_{i}\left(R^{\prime}, \Omega-U_{k}(e)\right) \text { and } \varphi_{i}^{a}(\bar{e})=U_{i}\left(R^{\prime}, \Omega-\varphi_{k}^{a}(\bar{e})\right) .
$$

Since the uniform rule is one-sided resource-monotonic and $\left(R^{\prime}, \Omega-U_{k}(e)\right),\left(R^{\prime}, \Omega-\right.$ $\left.\varphi_{k}^{a}(\bar{e})\right) \in \mathcal{E}^{N \backslash\{k\}}$ are such that $\sum_{N \backslash\{k\}} p\left(R_{i}\right) \leq \Omega-\varphi_{k}^{a}(\bar{e}) \leq \Omega-U_{k}(e)$, it follows that for all $i \in N \backslash\{k\}$,

$$
U_{i}\left(R^{\prime}, \Omega-\varphi_{k}^{a}(\bar{e})\right) R_{i} U_{i}\left(R^{\prime}, \Omega-U_{k}(e)\right) .
$$


Hence, (2.9) and (2.10) imply (ii).

Case 3: $e \in \mathcal{A}^{N}, \bar{e} \notin \mathcal{A}^{N}$. Similar to Case 2 by interchanging the roles of $\bar{R}$ and $R$. Case 4: $e \in \mathcal{A}^{N}, \bar{e} \in \mathcal{A}^{N}$. Without loss of generality we assume that $\bar{R}$ is a $k$ deviation from $R$ such that $p\left(\bar{R}_{k}\right) \geq p\left(R_{k}\right)$. Hence, $\sum_{N} p\left(R_{i}\right) \leq \sum_{N} p\left(\bar{R}_{i}\right)<\Omega$ and there exists $j \in N$ such that $p\left(R_{j}\right)>\frac{\Omega}{2}$ and $p\left(\bar{R}_{j}\right)>\frac{\Omega}{2}$. Let $R^{\prime}=R_{N \backslash\{j\}} \in \mathcal{R}^{N \backslash\{j\}}$ and $\tilde{R}^{\prime}=\tilde{R}_{N \backslash j\}} \in \mathcal{R}^{N \backslash\{j\}}$. Then,

$$
\begin{aligned}
& \varphi_{i}^{a}(e)= \begin{cases}p\left(R_{j}\right)+a(e) & \text { if } i=j_{,} \\
U_{i}\left(R^{\prime}, \Omega-\varphi_{j}^{a}(e)\right) & \text { if } i \in N \backslash\{j\},\end{cases} \\
& \varphi_{i}^{a}(\bar{e})= \begin{cases}p\left(\bar{R}_{j}\right)+a(\bar{e}) & \text { if } i=j_{,} \\
U_{i}\left(\bar{R}^{\prime}, \Omega-\varphi_{j}^{a}(\bar{e})\right) & \text { if } i \in N \backslash\{j\} .\end{cases}
\end{aligned}
$$

Case 4.1: $k=j$. Suppose $\varphi_{j}^{a}(e) \leq \varphi_{j}^{a}(\tilde{e})$. Since the uniform rule is one-sided resourcemonotonic and $\left(R^{\prime}, \Omega-\varphi_{j}^{a}(e)\right),\left(\bar{R}^{\prime}, \Omega-\varphi_{j}^{a}(\bar{e})\right) \in \mathcal{E}^{N \backslash\{j\}}$ are such that $\Sigma_{N \backslash\{j\}} p\left(R_{i}\right) \leq$ $\Omega-\varphi_{j}^{a}(\bar{e}) \leq \Omega-\varphi_{j}^{a}(e)$, it follows that for all $i \in N \backslash\{j\}$,

$$
U_{i}\left(\bar{R}^{\prime}, \Omega-\varphi_{j}^{a}(\bar{e})\right) R_{i} U_{i}^{\prime}\left(R^{\prime}, \Omega-U_{j}(e)\right) .
$$

Hence, (2.11) and (2.12) imply (ii).

Suppose $\varphi_{j}^{a}(e) \geq \varphi_{j}^{a}(\bar{e})$. Then, by similar arguments as for the opposite inequality, one-sided resource-monotonicity implies (i).

Case 4.2: $k \neq j$. Since, $p\left(\bar{R}_{k}\right) \geq p\left(R_{k}\right)$ it follows that $s_{j}(e) \geq s_{j}(\bar{e})$. Hence, $a(e) \geq$ $a(\bar{e})$ and $\varphi_{j}^{a}(\bar{e}) \leq \varphi_{j}^{a}(e)$. Thus,

$$
\varphi_{j}^{a}(\ddot{e}) R_{j} \varphi_{j}^{a}(e) .
$$

Now, we have either

(a) $a(\bar{e})=s_{j}(\bar{e})$ or

(b) $a(\bar{e})=r_{j}\left(\frac{\Omega}{2}\right)-p\left(R_{j}\right)$.

Suppose (a) holds. Hence, for all $i \in N \backslash\{j\}, \varphi_{i}^{a}(\bar{e})=p\left(R_{i}\right)$. Thus, for all $i \in$ $N \backslash\{j, k\}$,

$$
\varphi_{i}^{a}(\bar{e}) R_{i} \varphi_{i}^{a}(e)
$$

Hence, (2.13) and (2.14) imply (ii).

Suppose (b) holds. Hence, $a(\bar{e})=a(e)$ and $\Omega-\varphi_{j}^{a}(\bar{e})=\Omega-\varphi_{j}^{a}(e)$. Since the uniform rule satisfies one-sided replacement-domination and $\left(R^{\prime}, \Omega-\varphi_{j}^{a}(e)\right),\left(\bar{R}^{\prime}, \Omega-\varphi_{j}^{a}(\bar{e})\right) \in$

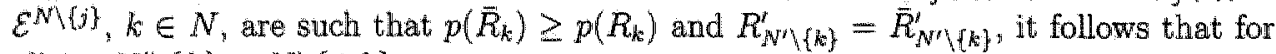
all $i \in N^{\prime} \backslash\{k\}=N \backslash\{j, k\}$,

$$
U_{i}\left(\bar{R}^{\prime}, \Omega-\varphi_{j}^{a}(\bar{e})\right) R_{i} U_{i}\left(R^{\prime}, \Omega-\varphi_{j}^{a}(e)\right)
$$

Thus, by (2.11) and (2.15), for all $i \in N \backslash\{j, k\}$,

$$
\varphi_{i}^{a}(\bar{e}) R_{i} \varphi_{i}^{a}(e)
$$

Hence, (2.13) and (2.16) imply (ii).

Now, Lemmas 2.1 and 2.2, together with Example 2.1 establish the answers to Questions 1 and 2. 


\section{Chapter 3}

\section{Fair allocation and single-dippedness ${ }^{1}$}

We consider the allocation, or division; of some perfectly divisible commodity among a group of agents with single-dipped preferences. This means that each agent has a worst share, his dip, below which and above which his welfare is increasing. For example, consider two types of work which have negative cross-effects like, perhaps, teaching and management in aniversity: combinations of the two types of work may be less preferred than one-type tasks. Yet another example is a two-goods exchange economy with fixed prices and strictly quasi-convex utility functions, which induce single-dipped preferences on the budget lines.

In a public good context Vickrey (1960) refers to the class of single-dipped preferences as "single-troughed" preferences and mentions that this restricted domain has been much less studied than the single-peaked domain. Inada (1964) studies singledipped preferences over triples of alternatives. ${ }^{2}$ Peremans and Storcken (1997) consider the problem of locating a public good with strongly negative externalities. These externalities induce single-dipped preferences on the set of admissible locations: To the best of our knowledge, single-dippedness has only been considered for public good situations.

In Chapter 2 we reviewed the literature on fair allocation when preferences are single-peaked. The uniform rule turned out to satisfy many appealing properties such as Pareto optimality, strategy-proofness, no-envy, anonymity, one-sided populationmonotonicity, one-sided replacement-domination, and consistency.

As we will show in this chapter, assuming in a private good context that preferences are single-dipped instead of single-peaked changes the results significantly. It turns out that the whole unit must be assigned to a single agent if the allocation rulle satisfies Pareto optimality, strategy-proofness, and one of the properties replacement domination, consistency, and population-monotonicity. Furthermore, if we impose Pareto optimality, other conditions such as anonymity or no-envy are generally incompatible.

This chapter is organized as follows. In Section 3.1 we introduce the model and give

${ }^{1}$ This chapter is based on Klaus, Peters, and Storcken (1997a) and Klaus (1997a).

${ }^{2}$ In Inada (1964) single-dipped preferences are called single-caved. 
a description of all Pareto optimal allocations for a given economy. In Section 3.2 we prove that for a fixed set of agents an allocation rule that satisfies Pareto optimality, strategy-proofness; and replacement-domination always assigns the whole amount of the commodity to a single agent. Using this result, we derive a characterization of all allocation rules satisfying these properties. In Section 3.3 , this characterization is extended to a variable population setting. In addition, replacing replacement-domination by either consistency or population-monotonicity yield similar characterizations. Remarkably, with only minor adjustments in notation, the characterizations we obtain here are also valid for economies where one indivisible commodity has to be allocated among a group of agents. Since here we are concerned with the allocation of an infinitely divisible commodity, we postpone these results to Appendix A. In Section 3.4 we establish the independence of the properties in the characterizations. Furthermore, we analyze the compatibility of Pareto optimality with other properties, mainly anonymity, no-envy, equal-treatment, and converse consistency.

\subsection{The model}

The allocation model for agents with single-dipped preferences is essentially the same as the allocation model for agents with single-peaked preferences we introduced in Chapter 2. In order to keep this chapter self-contained, we reintroduce the whole model and restate the central properties we explore. However, for more details concerning the motivation and the background of the properties we analyze in this section, we refer to Chapter 2.

We start our analysis of this new class of economies with the following fixed population set-up. One unit of some infinitely divisible commodity has to be divided among a set $N=\{1, \ldots, n\}$ of agents. ${ }^{3}$ Each agent $i \in N$ is equipped with a single-dipped preference relation $R_{i}$ defined over the closed interval $[0,1]{ }^{4}$ Single-dippedness of $R_{i}$ means that there exists a point $d\left(R_{i}\right) \in[0,1]$, called the dip of agent $i$, with the following property: for all $x, y \in[0,1]$ with $x<y \leq d\left(R_{i}\right)$ or $x>y \geq d\left(R_{i}\right)$ we have $x P_{i} y^{5}$

By $\mathcal{D}$ we denote the class of all single-dipped preference relations over $[0,1]$. Let $R=\left(R_{1}, \ldots, R_{n}\right) \in \mathcal{D}^{N}$ be a (preference) profile. Since, for the time being, the set of agents and the amount to divide are fixed we simply designate an economy by a profile of preference relations. Thus, the class of economies equals $\mathcal{D}^{N}$.

Let $\chi^{1}, \chi^{2}, \ldots, \chi^{n}$ denote the unit vectors in $\mathbb{R}^{N}$, where $\chi_{j}^{i}=1$ if $j=i$ and $\chi_{j}^{i}=0$ if $j \neq$. Let $X=\left\{\chi^{1}, \ldots, \chi^{n}\right\}$ and denote by $\mathcal{X}$ its convex hull. Note that $\mathcal{X}$ equals the $(n-1)$-dimensional unit simplex. Then, a feasible allocation for $R \in \mathcal{D}^{N}$ is a vector $x \in X^{X}$. An allocation rule $\Phi$, or a rule for short, is a function that assigns to every

\footnotetext{
${ }^{3}$ Note that free disposal of the commodity is not allowed.

"Without loss of generality, we may assume that the agents" preference relations are continuous as well:

${ }^{5}$ As usual, $x R_{i} y$ is interpreted as " $x$ is weakly preferred to $y$ ", and $x P_{i} y$ as " $x$ is strictly preferred to $y^{\prime \prime}$. Furthermore, $x I_{i} y$ means that agent $i$ is indifferent between $x$ and $y$.
} 
$R \in \mathcal{D}^{N}$ a feasible allocation, denoted $\Phi(R)$. Given $i \in N$, we call $\Phi_{i}(R)$ the share of agent $i$.

As in Chapter 2, we are interested in rules that assign Pareto optimal allocations: an allocation $y \in \mathcal{X}$ is Pareto optimal if there is no feasible allocation $x \in \mathcal{X}$ such that for all $i \in N, x_{i} R_{i} y_{i}$, and for some $j \in N, x_{j} P_{j} y_{j}$.

Pareto optimality: For all $R \in \mathcal{D}^{N}$, there is no feasible allocation $x \in \mathcal{X}$ such that for all $i \in N, x_{i} R_{i} \Phi_{i}(R)$, and for some $j \in N, x_{j} P_{j} \Phi_{j}(R)$.

Just as in the model with single-peaked preferences (see Section 2.1, page 19), there is a simple description of Pareto optimality for our model. For this description we introduce the following "partition" of the agents. For an economy $R \in \mathcal{D}^{N}$ denote by $N_{+}(R)=\left\{i \in N \mid \mathbb{1} P_{i} 0\right\}$ the set of agents who strictly prefer 1 to 0, by $N_{0}(R)=\{i \in$ $\left.N \mid 0 I_{i} 1\right\}$ the set of agents who are indifferent between 0 and 1 , and by $N_{-}(R)=$ $\left\{i \in N \mid 0 P_{i} 1\right\}$ the set of agents who strictly prefer 0 to 1 .

Hence, for all $R \in \mathcal{D}^{N}, N=N_{+}(R) \cup N_{0}(R) \cup N_{-}(R)$ and the sets $N_{+}(R), N_{0}(R)$, and $N_{-}(R)$ are pairwise disjoint. ${ }^{6}$

Lemma 3.1 A rule $\Phi$ is Pareto optimal if and only if for all $R \in \mathcal{D}^{N}$ the following holds.

Case 1 : If $N_{+}(R) \neq \emptyset$, then

for all $i \notin N_{+}(R), \Phi_{i}(R)=0$ and

for all $i \in N_{+}(R)$, either $\Phi_{i}(R)=0$ or $\Phi_{i}(R) P_{i} 0{ }^{7}$

Case 2: If $N_{+}(R)=\emptyset$ and $N_{0}(R) \neq \emptyset$, then

for some $j \in N_{0}(R), \Phi(R)=\chi^{j}$.

Case $3:$ If $N_{-}(R)=N$, then

for all $i \in N$, either $\Phi_{i}(R)=1$ or $\Phi_{i}(R) P_{i} 1^{8}$

Proof. Let $\Phi$ be a rule and let $R \in \mathcal{D}^{N}$. It is easy to show that all allocations described in Lemma 3.1 are Pareto optimal. It remains to show that they are the only Pareto optimal allocations. Let $\Phi$ be Pareto optimal and let $y=\Phi(R)$.

Case 1: $N_{+}(R) \neq \emptyset$. Suppose, by contradiction, that $\bar{N}=\left\{i \in N \mid\right.$ either $\left[i \notin N_{+}(R)\right.$ and $\left.y_{i} \neq 0\right]$ or $\left[i \in N_{+}(R)\right.$ and $\left.\left.y_{i} \neq 0,0 R_{i} y_{i}\right]\right\} \neq 0$.

Suppose that there exists an agent $j \in N_{+}(R) \backslash \bar{N}$ such that $y_{j} \neq 0$. Then, define $x \in \mathcal{X}$ as follows. For $i \in \bar{N}, x_{i}=0$. For $i \neq \bar{N}$ and $i \neq j, x_{i}=y_{i}$. Finally; $x_{j}=y_{j}+\sum_{\bar{N}} y_{i}$. The allocation $x$ is a Pareto improvement over $y$ : for all $i \in N$, $x_{i} R_{i} y_{i}$ and $x_{j} P_{j} y_{j}$.

Suppose that there exists no agent $j \in N_{+}(R) \backslash \bar{N}$ such that $y_{j} \neq 0$. Then, let $k \in N_{+}(R) \subseteq \bar{N}$ and $x=\chi^{k}$. The allocation $x$ is a Pareto improvement over $y$ : for all $\dot{i} \in N, x_{i} R_{i} y_{i}$ and $x_{k} P_{k} y_{k}$.

\footnotetext{
${ }^{6}$ However, since some of the sets $N_{4}(R), N_{0}(R)$, and $N_{-}(R)$ are possibly empty, strictly speaking, $\left\{N_{+}(R), N_{0}(R), N_{-}(R)\right\}$ may not constitute a partition.

'Tsee Figure 3.1 (a).

${ }^{8}$ See Figure 3.1 (b).
} 


$$
i, j \in N_{+}(R)
$$
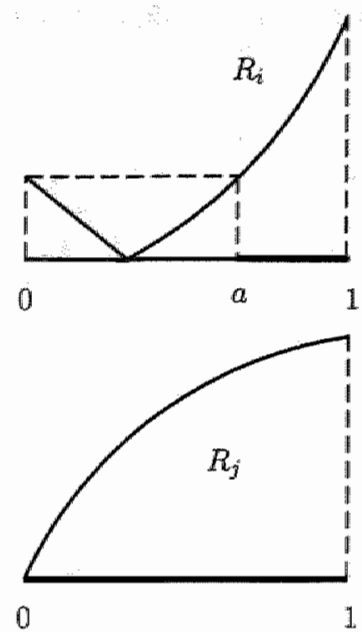

(a) $k, l \in N_{-}(R)$
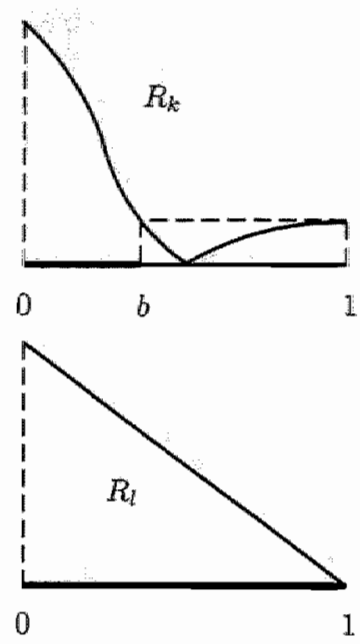

(b)

Figure 3.1: Some Pareto optimal shares as described in Lemma 3.1.

(a) Lemma 3.1, Case 1: $N_{+}(R) \neq 0$. Possible Pareto optimal shares for agents $i, j \in$ $N_{+}(R), x_{i}, x_{j}$, are : $x_{i} \in\{0\} \cup(a, 1]$ and $x_{j} \in[0,1]$.

(b) Lemma 3.1, Case 3: $N_{-}(R)=N$. Possible Pareto optimal shares for agents $k, l \in N_{-}(R), y_{k}, y_{l}$, are $: y_{k} \in[0, b) \cup\{1\}$ and $y_{l} \in[0,1]$.

Case 2: $N_{*}(R)=\emptyset$ and $N_{0}(R) \neq \emptyset$. It is easy to see that for all $j \in N_{0}(R)$, $k \in N_{-}(R), \chi^{j}$ Pareto dominates $\chi^{k}$. Now, suppose by contradiction that $\bar{N}=\{i \in$ $\left.N \mid y_{i} \notin\{0,1\}\right\} \neq 0$. Let $j \in \bar{N}$. Then, $x^{j}$ is a Pareto improvement over $y$ : for all $i \in N_{y} \chi_{i}^{j} R_{i} y_{i}$ and $\chi_{j}^{j} P_{j} y_{j}$.

Case 3: $\quad N_{c o}(R)=N$. Suppose by contradiction that $\bar{N}=\left\{i \in N \mid y_{i} \neq 1\right.$ and $\left.1 R_{i} y_{i}\right\} \neq 0$. Let $j \in \vec{N}$ and $k \in N$ be such that $y_{k} \neq 0$. Then, $\chi^{j}$ is a Pareto

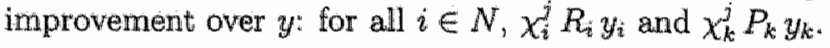

\subsection{Fixed population}

In this section, in addition to Pareto optimality, we are interested in strategy-proofness and replacement-domination. Strategy-proofness states that no agent ever benefits from misrepresenting his preferences (see also Section 2.2, page 22). Before we give the formal definition, we introduce some notation.

Let $N, M$ be such that $M \subseteq N$. Let $N \backslash M=\{i \in N \mid \dot{z} M\}$. For $R \in \mathcal{D}^{N}$ the 
restriction $\left(R_{i}\right)_{i \in M} \in \mathcal{D}^{M}$ of $R$ to $M$ is denoted by $R_{M}$. Let $R_{y} \bar{R} \in \mathcal{D}^{N}, i \in N$, and

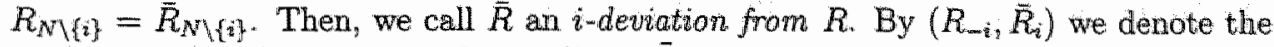
profile obtained from $R$ by replacing $R_{i}$ by $\bar{R}_{i}$.

Strategy-proofness: For all $R, \bar{R} \in \mathcal{D}^{N}$ and all $j \in N$, if $\bar{R}$ is a $j$-deviation from $R$, then $\Phi_{j}(R) R_{j} \Phi_{j}(\widetilde{R})$.

If under replacement-domination an agent's preference relation is "replaced" by some other admissible preference relation, then this unilateral change affects all remaining agents in the same direction, i.e., these agents all (weakly) gain or all (weakly) lose as a result of the replacement (see also Section 2.2 , page 24 ).

Replacement-domination: For all $R, \tilde{R} \in \mathcal{D}^{N}$ and all $j \in N$, if $\vec{R}$ is a $j$ deviation from $R$, then either [for all $i \in N \backslash\{j\}, \Phi_{i}(R) R_{i} \Phi_{i}(\bar{R})$ ] or [for all $i \in N \backslash\{j\}$, $\left.\Phi_{i}(\bar{R}) R_{i} \Phi_{i}(R)\right]$.

Note that replacement-domination is trivially satisfied if $|N|=2$.

The following example shows that the class of Pareto optimal and strategy-proof rules that does not satisfy replacement-domination also contains rules that rely on information that we do not consider as crucial for the problem at hand.

Example 3.1 The following rule $\tilde{\Phi}$ satisfies Pareto optimality and strategy-proofness. Without loss of generality, we define $\tilde{\Phi}(R)$ for $N=\{1,2,3\}$.

Case 1: $\quad N_{+}(R) \neq \emptyset$. If $N_{+}(R)=\{i\}$, then $\tilde{\Phi}(R)=\chi^{i}$.

If $\left|N_{+}(R)\right|>1$, then

$$
\tilde{\Phi}(R)= \begin{cases}\chi^{1} & \text { if } 1 P_{1} 0, \\ \chi^{2} & \text { if } 0 R_{1} 1 \text { and } d\left(R_{1}\right) \in \mathbb{Q}, \\ \chi^{3} & \text { if } 0 R_{1} 1 \text { and } d\left(R_{1}\right) \notin \mathbb{Q} .\end{cases}
$$

Case 2: $\quad N_{+}(e)=\emptyset$ and $N_{0}(e) \neq \emptyset$. Then $\tilde{\Phi}(R)=\chi^{i}, i \in N_{0}(e)$.

Case 3: $\quad N=N_{-}(e)$. Then

$$
\tilde{\Phi}(R)= \begin{cases}\chi^{2} & \text { if } d\left(R_{1}\right) \in \mathbb{Q} \\ \chi^{3} & \text { if } d\left(R_{1}\right) \notin \mathbb{Q}\end{cases}
$$

For an example of a rule that satisfies Pareto optimality, strategy-proofness, but not replacement-domination and that does not always assign the whole unit of the commodity to a single agent, we refer to Section 3.4, Example 3.6.

Next, we show that a rule $\Phi$ that satisfies Pareto optimality, strategy-proofness, and replacement-domination assigns the whole unit to a single agent.

Let $\mathcal{B}_{\Phi}=\left\{R \in \mathcal{D}^{N} \mid \Phi(R) \notin X\right\}$ be the set of economies that yield a broken allocation under $\Phi$, i.e., a feasible allocation where no agent obtains the whole unit. 
Lemma 3.2 Let $\Phi$ be a rule that satzsfies Pareto optimality, strategy-proofness, and replacement-domination. Then, $\mathcal{B}_{\text {舟 }}=\emptyset$.

Proof. Let $\Phi$ satisfy the properties listed in the lemma and suppose, by contradiction, that $\mathcal{B}_{\Phi} \neq \emptyset$.

Because $\mathcal{B}_{\Phi} \neq \emptyset$ and $N$ is finite, there exists a profile $R \in \mathcal{B}_{\Phi}$ with a maximal number of agents who strictly prefer 0 to 1 . So, let $R$ be broken under $\Phi$ and for all $R^{r} \in \mathcal{B}_{\tilde{Q}},\left|N_{-}(R)\right| \geq\left|N_{-}\left(R^{\prime}\right)\right|$. Let $B_{\Phi}(R)$ denote the set of agents with broken shares at $\Phi(R)$, i.e., $B_{\Phi}(R)=\left\{i \in N \mid \Phi_{i}(R) \notin\{0,1\}\right\}$. Hence, $B_{\Phi}(R) \neq \emptyset$ and $\left|B_{\Phi}(R)\right| \geq 2$.

Because the sets $N_{+}(R), N_{0}(R)$, and $N_{-}(R)$ constitute a partition of $N$, one of the following cases occurs.

Case 1: $\quad N_{+}(R) \neq \emptyset$. By Lemma 3.1, it follows that $B_{\Phi}(R) \subseteq N_{+}(R)$.

Let $i \in B_{\Phi}(R)$ and $\left(R_{-i}, \bar{R}_{i}\right)$ be an $i$-deviation from $R$ such that $i \in N_{-}\left(R_{-i}, \bar{R}_{i}\right)$. Because the number of agents in $N_{-}(R)$ is maximal for $R \in \mathcal{B}_{\Phi}$, it follows that $\left(R_{-i}, \bar{R}_{i}\right) \notin \mathcal{B}_{\Phi}$. So, by Pareto optimality and $\left|B_{\Phi}(R)\right| \geq 2$, there is $j \in N_{+}(R)$ such that $\Phi\left(R_{-i}, \bar{R}_{i}\right)=\chi^{j}$. By strategy-proofness, $j \neq i$. Replacement-domination and $B_{\Phi}(R) \subseteq N_{+}(R)$ now imply $B_{\Phi}(R)=\{i, j\}$.

Next, consider an $i$-deviation $R^{1}$ from $R$, a $j$-deviation $R^{2}$ from $R$, and an $i$-deviation $R^{3}$ from $R^{2}$, such that $1 P_{i}^{1} 0 P_{i}^{1} \Phi_{i}(R), 1 P_{j}^{2} 0 P_{j}^{2} \Phi_{j}(R)$, and $R_{i}^{3}=R_{i}^{1}$.

Strategy-proofness for the $i$-deviation $R^{1}$ from $R$ implies $\Phi_{i}(R) \geq \Phi_{i}\left(R^{1}\right)$.

By $N_{+}(R)=N_{+}\left(R^{1}\right)$ and Lemma 3.1 it follows that either $\Phi_{i}\left(R^{1}\right)=0$ or $\Phi_{i}\left(R^{1}\right) P_{i}^{1} 0$. The latter can only be true if $\Phi_{i}\left(R^{\mathbb{1}}\right)>\Phi_{i}(R)$, which contradicts strategy-proofness. Hence, $\Phi_{i}\left(R^{1}\right)=0$. Note, that $R^{1}$ is an $i$-deviation from $\left(R_{-i}, \bar{R}_{i}\right)$. Thus, by $\Phi\left(R_{-i}, \bar{R}_{i}\right)=$ $\chi^{j}$ and replacement-domination, it follows that $\Phi\left(R^{1}\right)=\chi^{j}$. By strategy-proofness for the $j$-deviation $R^{3}$ from $R^{1}$, we have $\Phi\left(R^{3}\right)=\chi^{j}$. Similarly, by interchanging the roles of $i$ and $j$, it follows that $\Phi\left(R^{3}\right)=\chi^{i}$, which is a contradiction.

Case 2: $\quad N_{+}(R)=\emptyset$ and $N_{0}(R) \neq \emptyset$. By Lernma 3.1, $\Phi(R)=\chi^{j}$ for some $j \in N_{0}(R)$. This contradicts $B_{\Phi}(R) \neq \emptyset$.

Case 3: $N_{-}(R)=N$. Let $\{i, j\} \in B_{\Phi}(R)$. Consider an $i$-deviation $R^{1}$ from $R$, a $j$-deviation $R^{2}$ from $R_{3}$ and an $i$-deviation $R^{3}$ from $R^{2}$, such that $0 P_{i}^{1} 1 P_{i}^{1} \Phi_{i}(R)$, $0 P_{j}^{2} \mathbb{1} P_{j}^{2} \Phi_{j}(R)$, and $R_{i}^{3}=R_{i}^{\mathbb{1}}$.

Strategy-proofness for the $i$-deviation $R^{1}$ implies $\Phi_{i}(R) \leq \Phi_{i}\left(R^{1}\right)$.

By $N_{-}\left(R^{1}\right)=N$ and Lemma 3.1, it follows that either $\Phi_{i}\left(R^{1}\right)=1$ or $\Phi_{i}\left(R^{1}\right) P_{i}^{1} 1$. The latter can only be true if $\Phi_{i}(R)>\Phi_{i}\left(R^{1}\right)$, which contradicts strategy-proofness. Hence, $\Phi\left(R^{1}\right)=\chi^{i}$. By strategy-proofness and replacement-domination, for the $j$ deviation $R^{3}$ from $R^{1}$, we have $\Phi\left(R^{3}\right)=\chi^{i}$. Similarly, by interchanging the roles of $i$ and $j$, it follows that $\Phi\left(R^{3}\right)=\chi^{j}$, which is a contradiction.

Lemma 3.2 states that for a rule that satisfies Pareto optimality, strategy-proofness, and replacement-domination, the whole unit is assigned to a single agent. The selection of this agent can be described as follows.

Fix a linear order of the set of agents. We ask the agent who is first in this order whether he prefers 1 to 0 . If he prefers the whole unit, he receives it and we are done. 
If not, we ask the second agent the same question; etc. Hence, the first agent according to the fixed order who prefers the whole unit to nothing receives it:

If no agent strictly prefers 1 to 0 and at least one agent is indifferent between 0 and 1 , then the unit can be assigned "arbitrarily" to any of the indifferent agents.

If all agents strictly prefer 0 to 1 , there exists a preselected agent who receives it. Loosely speaking, there is a "poor guy" who always receives the whole unit when nobody else wants it.

For a formal description, we introduce the following notation.

A permutation $\pi$ on $N$ is a bijective function $\pi \cdot N \rightarrow N$. By $\mathbb{I}^{N}$ we denote the set of all permutations on $N$. Since for $\pi \in \Pi^{N}$ and $i \in N, \pi(i)$ can also be interpreted as the position of $i$ in a linear order of $N$, we alternatively refer to $\pi$ as to the linear order on $N$. By $G^{N}$ we denote the set of choice functions $g: \mathcal{D}^{N} \rightarrow N$ such that $g(R) \in N_{0}(R)$ if $N_{0}(R) \neq \emptyset$.

Selection $f_{\pi, g, k}: \quad$ Let $\pi \in \Pi^{N}, g \in G^{N}$, and $k \in N$. Then the selection $f_{\pi, g, k}: \mathcal{D}^{N} \rightarrow$ $N$ (based on $\pi, g$, and $k$ ) is defined as follows. For all $R \in \mathcal{D}^{N}$ :

Case 1: If $N_{+}(R) \neq \emptyset$, then $f_{\pi, g, k}(R)=\arg \min \left\{\pi(i) \| i \in N_{+}(R)\right\}$.

Case 2: If $N_{+}(R)=\emptyset$ and $N_{0}(R) \neq \emptyset$, then $f_{\pi, g, k}(R)=g(R)$.

Case 3: If $N=N_{-}(R)$, then $f_{\pi, g, k}(R)=k$.

Now, we can characterize all rules that satisfy Pareto optimality, strategy-proofness, and replacement-domination.

Theorem 3.1 A rule $\Phi$ satisfies Pareto optimality; strategy-proofness, and replacement-domination if and only if there exist $\pi \in \Pi^{N}, g \in G^{N}$, and $k \in N$ such that for all $R \in \mathcal{D}^{N}$,

$$
\Phi(R)=\chi^{f \pi_{, g, k}(R)}
$$

Proof. Let $\pi \in \Pi^{N}, g \in G^{N}$, and $k \in N$ be such that for all $R \in \mathcal{D}^{N}, \Phi(R)=\chi^{f_{\pi, g, k}(R)}$. We prove that $\Phi$ satisfies Pareto optimality, strategy-proofness, and replacement-domination.

By Lemma 3.1 it follows that $\Phi(R)=\chi^{f_{\pi_{g, g, k}}(R)}$ is a Pareto optimal allocation.

Next, consider $R \in \mathcal{D}^{N}$ and an $z$-deviation $R$ from $R$. In order to prove strategyproofness we consider the following three cases.

Case 1: $i \in N_{+}(R)$. Hence, $N_{+}(R) \neq \emptyset$ and $\Phi(R)=\chi^{\arg \min \left\{\pi(j) \mid j \in N_{+}(R)\right\}}$. Let $l=$ $\arg \min \left\{\pi(j) \| j \in N_{+}(R)\right\}$. If $i \neq l$, then $\arg \min \left\{\pi(j) \mid j \in N_{+}(R)\right\}=\arg \min \{\pi(j) \mid$ $\left.j \in N_{+}(\bar{R})\right\}$. Hence, $\Phi(R)=\Phi(\bar{R})$. If $i=l$, then either $\Phi_{i}(R)=\Phi_{i}(\vec{R})=1$ or $\Phi_{i}(\bar{R})=0$. Hence, $\Phi_{i}(R) R_{i} \Phi_{i}(\bar{R})$.

Case 2: $i \in N_{0}(R)$. Because, by the definition of $\Phi$, the whole unit is assigned to one of the agents in $N$ and agent $i$ is indifferent between receiving the whole unit and receiving nothing, $\Phi_{i}(R) R_{i} \Phi_{i}(\vec{R})$.

Case 3: $i \in N_{-}(R)$. If $\Phi_{i}(R)=0$, agent $i$ cannot improve his outcome by unilateral deviation. If $\Phi_{i}(R)=1$, then by Pareto optimality, $N=N_{-}(R)$. So, $i$ equals the 
preselected agent of the selection $f_{\pi, g, k}, i_{n} e_{,}, \dot{z}=k$. Thus, if $N_{-}(\bar{R})=N_{*} \Phi_{i}(R)=1$. For $N_{-}(\bar{R}) \neq N, \Phi_{i}(\bar{R})=1$ follows by Pareto optimality. Hence, $\Phi_{i}(R) R_{i} \Phi_{i}(\bar{R})$.

Now, by Cases 1,2 , and 3 it follows that for all $i$-deviations $\bar{R}$ from $R, \Phi_{i}(R) R_{i} \Phi_{i}(\bar{R})$. This proves strategy-proolness.

Let $R \in \mathcal{D}^{N}$ and consider an $i$-deviation $\bar{R}$ from $R$. In order to prove replacementdomination we assume by contradiction that agents $l, m \neq i$ exist such that $\Phi_{l}(R) P_{l} \Phi_{l}(\bar{R})$ and $\Phi_{m}(\tilde{R}) P_{m} \Phi_{m}(\tilde{R})$.

Because $\Phi$ assigns the whole amount to one of the agents, this can only occur if either $\left[l, m \in N_{+}(R)\right.$ such that $\Phi_{l}(R)=\Phi_{m}(\bar{R})=1$ and $\left.\Phi_{l}(\bar{R})=\Phi_{m}(R)=0\right]$ or $\left[l, m \in N_{-}(R)\right.$ such that $\Phi_{l}(R)=\Phi_{m}(\bar{R})=0$ and $\left.\Phi_{l}(\bar{R})=\Phi_{m}(R)=1\right]$. However, by the definition of $\Phi$ by means of the selection $f_{\pi, g_{s} k}$, none of these changes can occur after a unilateral change $\bar{R}$ of an agent $i \neq l, m$. Hence, $\Phi$ satisfies replacement-domination.

The proof of the remaining part of the theorem is constructive. Suppose $\Phi$ is a rule satisfying the properties listed in the theorem. Then, we construct a selection $f_{\pi, g, k}: \mathcal{D}^{N} \rightarrow N$ based on (a) a permutation $\pi \in \Pi^{N},\left(\right.$ b) a choice function $g: \mathcal{D}^{N} \rightarrow N$, and (c) an individual $k \in N$, such that for all $R \in \mathcal{D}^{N}, \Phi(R)=\chi^{f_{\pi, g, k}(R)}$.

By Lemma 3.2 it follows that $\mathcal{B}_{\Phi}=\emptyset$. Hence, for all $R \in \mathcal{D}^{N}, \Phi(R)=\chi^{i}$ for some $i \in N$.

(a) Definition of $\pi \in \mathbb{I}^{N}$.

(a.1) Let $R \in \mathcal{D}^{N}$ be such that $N_{+}(R)=N$. Then, $\Phi(R)=\chi^{i_{1}}$ for some $i_{\mathrm{I}} \in N$. Set $\pi\left(i_{1}\right)=1$. By strategy-proofness, for all $i_{1}$-deviations $R^{\prime}$ from $R$ with $i_{1} \in N_{+}\left(R^{\prime}\right)$.

$$
\Phi\left(R^{\prime}\right)=\chi^{i_{1}}
$$

Let $j \neq i_{1}$ and consider a $j$-deviation $R^{\prime \prime}$ from $R$. By strategy-proofness and replacementdomination,

$$
\Phi\left(R^{\prime \prime}\right)=\chi^{i_{1}}
$$

Now, applying (3.1) and (3.2) repeatediy, it follows that for all profiles $\bar{R} \in \mathcal{D}^{N}$ with $i_{1} \in N_{+}(\bar{R}) \subseteq N$

$$
\Phi(\bar{R})=\chi^{i_{1}}
$$

(a.2) Next, let $R \in \mathcal{D}^{N}$ be such that $N_{+}(R)=N^{1}:=N \backslash\left\{i_{1}\right\}$. Then, $\Phi(R)=\chi^{i_{2}}$ for some $i_{2} \in N^{1}$. Set $\pi\left(i_{2}\right)=2$. Similarly as in (a.1) it can be shown that for all profiles $\bar{R} \in \mathcal{D}^{N}$ with $\dot{r}_{2} \in N_{+}(\bar{R}) \subseteq N^{1}$,

$$
\Phi(\tilde{R})=\chi^{i_{2}}
$$

(a.3) Let $R \in \mathcal{D}^{N}$ be such that $N_{+}(R)=N^{2}:=N^{1} \backslash\left\{i_{2}\right\}$. Then $\Phi(R)=\chi^{i_{3}}$ for some $i_{3} \in N^{2}$. Set $\pi\left(i_{3}\right)=3$. etc.

It is now clear how the linear order $\pi \in \Pi^{N}$ is constructed step by step by leaving out the agent receiving the whole unit. By the definition of $\pi$, it follows that for all $R \in \mathcal{D}^{N}$ such that $N_{+}(R) \neq \emptyset$.

$$
\Phi(R)=\chi^{\arg \min \left\{(x)(g) \mid i \in N_{+}(R)\right\}} .
$$


(b) Definition of $g: D^{N} \rightarrow N$.

Define a choice function $g: \mathcal{D}^{N} \rightarrow N$ as follows. For all $R \in \mathcal{D}^{N}$,

$$
g(R):=\left\{\begin{array}{cl}
i & \text { if } \Phi(R)=\chi^{i} \text { and } N_{+}(R)=\emptyset, \\
\min \left\{i \mid i \in N_{0}(R)\right\} & \text { if } N_{+}(R) \neq \emptyset \text { and } N_{0}(R) \neq \emptyset, \\
\min \left\{i \mid i \in N_{+}(R)\right\} & \text { if } N_{+}(R) \neq \emptyset \text { and } N_{0}(R)=\emptyset
\end{array}\right.
$$

Hence, by the definition of $g$, it follows that for all $R \in \mathcal{D}^{N}$ such that $N_{+}(R)=\emptyset$ and $N_{0}(R) \neq \emptyset$,

$$
\Phi(R)=\chi^{q(R)} .
$$

Note, that the choice function $g$ is not uniquely determined: (3.4) holds for all choice functions $\vec{g}: \mathcal{D}^{N} \rightarrow N$ such that $\bar{g}(R)=i$ if $\Phi(R)=\chi^{i}$ and $N_{+}(R)=\emptyset$.

(c) Definition of $k \in N$.

Let $R \in \mathcal{D}^{N}$ be such that $N_{-}(R)=N$. Then, $\Phi(R)=\chi^{i}$ for some $i \in N$. By strategy-proofness, it follows that for all $i$-deviations $R^{\prime}$ from $R$,

$$
\Phi\left(R^{t}\right)=\chi^{i}
$$

Let $j \neq i$ and consider a $j$-deviation $R^{\prime \prime}$ from $R$ with $N_{-}\left(R^{\prime \prime}\right)=N$. Then, by strategyproofness and replacement-donination,

$$
\Phi\left(R^{\prime \prime}\right)=\chi^{i} .
$$

Now, applying (3.5) and (3.6) repeatedly, it follows that for all $\ddot{R} \in \mathcal{D}^{N}$ with $N_{-}(\tilde{R})=$ $N$,

$$
\Phi(\bar{R})=\chi^{i}
$$

Hence, by setting $k=i$, for all $R \in \mathcal{D}^{N}$ with $N_{-}(R)=N$,

$$
\Phi(R)=\chi^{k}
$$

Now, equations (3.3), (3.4), and (3.7) imply that for all $R \in \mathcal{D}^{N}, \Phi(R)=\chi^{f_{\pi, Q, k}(R)}$.

Remark 3.1 Restricting our model to situations where agents are not indifferent between 0 and 1 and at least one agent prefers 1 to 0 , the class of rules that satisfy Pareto optimality, strategy-proofness, and replacement-domination reduces to the serial dictatorships described in Papai (1996) for the problem of allocating a single indivisible object to one agent in a group.

\subsection{Variable population}

Next, we extend the model and allow for changes in the set of agents as well as in the amount that has to be distributed. 
There is a finite population of potential agents, indexed by $\mathbb{P}^{9}$ Each agent $i \in \mathbb{P}$ is equipped with a single-dipped preference relation over the non-negative real numbers $\mathbb{R}_{+}{ }^{10}$ By $\mathcal{D}$ we now denote the class of all single-dipped preference relations over $\mathbb{R}_{+}$. By $\mathcal{P}$ we denote the class of non-empty subsets of $\mathbb{P}$. For $N \in \mathcal{P}, \mathcal{D}^{N}$ denotes the set of (preference) profiles $R=\left(R_{i}\right)_{i \in N}$ such that for all $i \in N, R_{i} \in \mathcal{D}$.

Now, an economy is formalized as follows. Let $\Omega \in \mathbb{R}_{+}$be the amount of an infinitely divisible commodity, or the (social) endowment, that has to be distributed among a group of agents $N \in \mathcal{P}$ with profile $R \in \mathcal{D}^{N}$. We call a pair $e=(R, \Omega) \in \mathcal{D}^{N} \times \mathbb{R}_{+}$an economy. Let $\mathcal{E}^{N}=\mathcal{D}^{N} \times \mathbb{R}_{+}$be the class of all economies. A feasible allocation for $e=(R, \Omega) \in \mathcal{E}^{N}$ is a vector $x \in \mathbb{R}_{+}^{N}$ such that $\sum_{N} x_{i}=\Omega$. An allocation rule $\varphi$, or a rule for short, is a function that assigns to every $N \in \mathcal{P}$ and every $e \in \mathcal{E}^{N}$ a feasible allocation, denoted $\varphi(e)$. As before, $\varphi_{i}(e)$ denotes the share of agent $i$.

The definitions of Pareto optimality, strategy-proofness, and replacement-domination can straightforwardly be adopted to this generalized model, and Theorem 3.1 can easily be extended. However, some extra notation is needed.

Let $N \in \mathcal{P}$. For $e=(R, \Omega) \in \mathcal{E}^{N}$, let $N_{+}(e)=\left\{i \in N \mid \Omega P_{i} 0\right\} ; N_{0}(e)$ and $N_{-}(e)$ are understood similarly. A selection $f_{\pi, g, k}^{N}: \mathcal{E}^{N} \rightarrow N$ can similarly be defined as before, by replacing $R$ by $e$ and $\mathcal{D}^{N}$ by $\mathcal{E}^{N}$. As before, for a given $N \in \mathcal{P}$ and $i \in N, \chi^{i}$ denotes the $i$-th unit vector of $\mathbb{R}^{N}$. Since we use the unit vectors in the description of allocations for a given economy, it is unambiguously determined to which "index set" of agents the unit vectors belong.

Corollary 3.1 A rule $\phi$ satisfies Pareto optimality, strategy-proofness, and replacement-domination if and only if for all $N \in \mathcal{P}$ and all $\Omega \in \mathbb{R}_{++}$, there exist $\pi \in \Pi^{N}$, $g \in G^{N}$, and $k \in N$ such that for all $e=(R, \Omega) \in \mathcal{E}^{N}$,

$$
\varphi(e)=\Omega x_{i, g, k}^{f_{n, k}^{N}(e)}
$$

Proof. For any fixed coalition $N \in \mathcal{P}$ and any fixed amount of the commodity $\Omega \in \mathbb{R}_{+}$: the proofs of Lemmas 3.1, 3.2, and Theorem 3.1 are similar as before by replacing the amount 1 by $\Omega, R$ by $e=(R, \Omega)$, and $\mathcal{D}^{N}$ by $\mathcal{E}^{N}$. This implies Corollary 3.1

Note that replacement-domination is trivially satisfied if $|N|=2$. For later reference, we state this as a corollary.

Corollary 3.2 Let $\varphi$ be a rule that satisfies Pareto optimality and strategy-proofness. Then, for all $N \in \mathcal{P},|N|=2$, and all $\Omega \in \mathbb{R}_{+}$, there exist $\pi \in \mathbb{\Pi}^{N}, g \in G^{N}$, and $k \in N$

\footnotetext{
${ }^{9}$ We also could choose an infinite population of potential agents that is represented by the natural numbers $\mathbb{N}$. However, then we would have to exoke some existence results from social choice theory to prove Theorems 3.2 and 3.3 instead of using constructive proofs. Since this would not only interrupt the flow of the exposition, but also increase the complexity of notation, here we decided to stick to the original constructive version of the proof. However, I wish to thank Ton Storcken for bringing the alternative approach to my attention.

${ }^{10}$ In Klaus, Peters, and Storcken (1996) we restrict the preferences to $[0,1]$.

Without loss of generality; we may assume that the agents' preference relations are continuous as well.
} 
such that for all $e=(R, \Omega) \in \mathcal{E}^{N}$,

$$
\varphi(e)=\Omega \chi^{f_{\pi, 8, k}^{N}(e)} .
$$

The next property we consider is consistency. Recall that for an economy $e=$ $(R, \Omega) \in \mathcal{E}^{N}$ consistency describes the stability of a recommended allocation $x \in \mathbb{R}_{+}^{N}$ in situations where a group of agents leave with their shares (see also Section 2.2 , page 26). Let $M \subseteq N$ and suppose that all agents in $N \backslash M$ leave with the shares assigned to them at $x$. Then, the shares assigned to the agents in $M$ at $x$ should be equal to the shares they receive for the reduced economy $\left(R_{M}, \sum_{M} x_{i}\right) \in \mathcal{E}^{M}$.

Let $M, N \in \mathcal{P}$ such that $M \subseteq N$. For $x \in \mathbb{R}^{N}$ the restriction $\left(x_{i}\right)_{i \in M} \in \mathbb{R}^{M}$ of $x$ to $M$ is denoted by $x_{M}$.

Consistency: For all $M, N \in \mathcal{P}$ with $M \subseteq N$ and all $e=(R, \Omega) \in \mathcal{E}^{N}, \varphi(e)_{M}=$ $\varphi\left(R_{M}, \sum_{M} \varphi_{i}(e)\right)$.

Next, we consider rules that satisfy Pareto optimality, strategy-proofness, and consistency. By Corollary 3.2 it follows that such a rule never assigns a broken allocation to a two-agent economy. Then, by consistency, it follows easily that a rule that satisfies the properties listed above always assigns the whole endowment to a single agent. Before we characterize the class of Pareto optimal, strategy-proof, and consistent rules, we show that these properties imply replacement-domination.

Lemma 3.3 Let $\varphi$ be a rule that satisfies Pareto optimality, strategy-proofness, and consistency. Then, $\varphi$ satisfies replacement-domination.

Proof. Let $\varphi$ satisfy the properties listed in the lemma. By Corollary 3.2 and feasibility, it follows that for all $N \in \mathcal{P}$ with $|N| \leq 2$ and all $e=(R, \Omega) \in \mathcal{E}^{N}$,

$$
\varphi(e)=\Omega \chi^{j}, \text { for some } j \in N \text {. }
$$

Suppose, by contradiction, that $\varphi$ does not satisfy replacement-domination. Then, there exist $S \in \mathcal{P},|S|>2, e=(R, \Omega), \bar{e}=(\bar{R}, \Omega) \in \mathcal{E}^{S}$, and $i, j, k \in S, j, k \neq i$, such that $R$ is an $i$-deviation from $\vec{R}$ and

$$
\varphi_{j}(e) P_{j} \varphi_{j}(\bar{e}) \text { and } \varphi_{k}(\bar{e}) P_{k} \varphi_{k}(e)
$$

By consistency of $\varphi$, this can only hold if $\varphi_{i}(e) \neq \varphi_{i}(\bar{e})$. Furthermore, $\varphi_{j}(e) \neq \varphi_{j}(\bar{e})$ and $\varphi_{k}(e) \neq \varphi_{k}(\bar{e})$. Hence, for two agents $l, m \in\{i, j, k\}$ it must be that either $\left[\varphi_{l}(e) \neq 0\right.$ and $\left.\hat{\varphi}_{m}(e) \neq 0\right]$ or $\left[\varphi_{l}(\bar{e}) \neq 0\right.$ and $\left.\varphi_{m}(\bar{e}) \neq 0\right]$.

Without loss of generality assume that $\varphi_{l}(e) \neq 0$ and $\varphi_{m}(e) \neq 0$. Now, consider the reduced economy $\tilde{e}=\left(R_{\{l, m\}}, \Sigma_{\{l, m\}} \varphi_{i}(e)\right) \in \mathcal{E}^{\{l, m\}}$. Then, by consistency, $\varphi(e)_{\{l, m\}}=$ $\varphi(\tilde{e})$. Hence,

$$
\varphi_{l}(\tilde{e}) \neq 0 \text { and } \varphi_{m}(\tilde{e}) \neq 0 \text {. }
$$

Since $\tilde{e}$ is a two-agent economy, this is in contradiction to (3.8). 
As already mentioned above, a rule that satisfies Pareto optimality; strategy-proofness, and consistency, assigns the social endowment to a single agent. Furthermore, the way that agent is selected should be "consistent across populations". Let the endowment $\Omega$ be fixed. Then, the selection of the agent can be described as follows. Let $N \in \mathcal{P}$ be a set of agents and $e=(R, \Omega) \in \mathcal{E}^{N}$ an economy.

By Pareto optimality the endowment $\Omega$ should, if possible, be allotted to an agent who prefers $\Omega$ to 0 . Among these agents, if there are several, the choice is made with respect a linear order $\pi_{+}$on $\mathbb{P}$, the set of potential agents.

We ask the first agent according to $\pi_{+}$who is a member of $N$, whether he prefers $\Omega$ to 0 . If he prefers the endowment, he receives it and we are done. If not, we ask the second agent according to $\pi_{+}$who is a member of $N$ the same question; etc. Hence, the first agent in the order $\pi_{+}$, who is in $N$ and prefers the endowment to nothing receives it.

If no agent in $N$ strictly prefers $\Omega$ to 0 and at least one agent in $N$ is indifferent between 0 and $\Omega$, then the endowment is assigned "arbitrarily" to an agent who is indifferent between 0 and $\Omega$. Again, we could make our choice with respect to a fixed order on $\mathbb{P}$ in order to satisfy the required properties. However, in this case, we have more degrees of freedom to specify a "consistent" choice so as to respect Pareto optimality and strategy-proofness (see Example 3.3).

Finally, if all agents in $N$ strictly prefer 0 to $\Omega$, then $\Omega$ is assigned to an agent with respect to a linear order $\pi_{-}$on $\mathbb{P}$. Now, the first agent in the order who is in $N$ receives the endowment.

For a formal description, we introduce the following notation.

Let $\Pi^{\mathbb{P}}$ denote the set of all permutations, or linear orders, on $\mathbb{P}$. We call $\mathcal{G}:=$ $\left\{g^{N} \in G^{N}\right\}_{N \in \mathcal{P}}$ a consistent collection of choice functions if for all $T^{\prime} \subseteq T \in \mathcal{P}$ and all $(R, \Omega) \in \mathcal{E}^{T}$ such that $g^{T}(R, \Omega) \in T^{\prime}$, it follows that $g^{T}(R, \Omega)=g^{T^{\prime}}\left(R_{T^{u}}, \Omega\right)$. By $\mathcal{C}^{\mathbb{P}}$ we denote the class of all consistent collections of choice functions. ${ }^{11}$

Consistent collection of selections $\left\{f_{\pi_{+}, G_{,} \pi_{-}}^{N}\right\}_{N \in \mathcal{P}}$ : Let $\pi_{+}, \pi_{-} \in \Pi^{\mathbb{P}}$ and $\mathcal{G}=$ $\left\{g^{N} \in G^{N}\right\}_{N \in \mathcal{P}} \in \mathcal{C}^{\mathbb{P}}$. Then, the consistent collection of selections $\left\{f_{\pi_{+}, G_{, \pi}, \pi_{-\infty}}^{N}\right\}_{N \in \mathcal{P}}$ (based on $\pi_{+}, \pi_{-}$, and $\left.\mathcal{G}\right)$ is defined as follows. For all $N \in \mathcal{P}$ and all $e=(R, \Omega) \in \mathcal{E}^{N}$ :

Case 1: If $N_{+}(e) \neq \emptyset$, then $f_{\pi_{+}, \mathcal{G}_{,} \pi_{-}}^{N}(e)=\arg \min \left\{\pi_{+}(i) \mid i \in N_{+}(e)\right\}$.

Case 2: If $N_{+}(e)=\emptyset$ and $N_{0}(e) \neq \emptyset$, then $f_{\pi_{+}, G_{,}, \pi_{-}}^{N}(e)=g^{N}(e)$.

Case 3: If $N_{-}(e)=N$, then $f_{N_{+}, g_{,} \pi_{-}}^{N}(e)=\arg \min \left\{\pi_{-}(i) \mid i \in N_{-}(e)\right\}$.

Theorem 3.2 A rule $\varphi$ satisfies Pareto optimality, strategy-proofness, and consistency if and only if for each $\Omega \in \mathbb{R}_{+}$there exist $\pi_{+}, \pi_{-} \in \mathbb{M}^{\mathbb{P}}$ and $\mathcal{G} \in \mathcal{C}^{\mathbb{P}}$ such that for

\footnotetext{
11Note that in the description of all Pareto optimal, strategy-proof, and consistent rules, we do not need this notion of consistency for the collection of choice functions in its full strengith; consistent choices only have to be made for all economies $\left(R_{,}, \Omega\right),\left(R_{T}, \Omega\right)$ such that $N_{+}(R, \Omega)=\emptyset$ and $N_{0}\left(R_{T^{*}}, \Omega\right) \neq 0$. However, since the choice functions are not uniquely determined, for notational convenience we require consistency of the collection of choice functions for all economies.
} 
all $N \in \mathcal{P}$ and all $e=(R, \Omega) \in \mathcal{E}^{N}$,

$$
\varphi(e)=\Omega \chi^{f_{\pi_{+}, g_{0} \pi_{-}}^{N}(e)} .
$$

Before we prove the theorem, we briefly discuss the difference between the rules described in Corollary 3.1 and in the previous theorem.

Let $N \in \mathcal{P}, e \in \mathcal{E}^{\mathbb{N}}$ and assume that the rule $\varphi$ satisfies Pareto optimality and strategy-proofness. If $\varphi$ satisfies consistency, then it belongs to the class of rules described in Theorem 3.2. By Lemma 3.3, consistency implies replacement-domination and $\varphi$ belongs to the class of rules described in Corollary 3.1.

Case 1: $\quad N_{+}(e) \neq 0$. A rule $\varphi$ that satisfies replacement-domination chooses an agent who prefers $\Omega$ to 0 with respect to an "arbitrarily" chosen linear order on $N$. To the contrary, a rule $\varphi$ that satisfies consistency chooses this agent with respect to a linear order on $N$ that is implied by a fixed linear order on the whole set of potential agents $\mathbb{P}$.

Case 2: $\quad N_{+}(e)=\emptyset$ and $N_{0}(e) \neq \emptyset$. A rule $\varphi$ that satisfies replacement-domination "arbitrarily" chooses a single agent who is indifferent between $\Omega$ and 0 . To the contrary, a rule $\varphi$ that satisfies consistency has to be consistent across populations.

Case 3: $\quad N_{-}(e)=N$. A rule $\varphi$ that satisfies replacement-domination, "arbitrarily" chooses a single agent who always receives the endowment if nobody else in $N$ wants it. To the contrary, a rule $\varphi$ that satisfies consistency chooses this agent with respect to an linear order on $N$ that is implied by a fixed linear order of the whole set of potential agents $\mathbb{P}$.

Proof. Let $\Omega \in \mathbb{R}_{+}$. Let $\pi_{+}, \pi_{-} \in \Pi^{\mathbb{P}}$ and $\mathcal{G}=\left\{g^{N} \in G^{N}\right\}_{N \in \mathcal{P}} \in \mathcal{C}^{\mathbb{P}}$ be such that for all $N \in \mathcal{P}$ and all $e=(R, \Omega) \in \mathcal{E}^{N}, \varphi(e)=\Omega \chi^{f_{\pi_{+}, \vartheta, \pi_{-}}^{N}(e)}$. As shown in the proof of Theorem 3.1, $\varphi$ satisfies Pareto-optimality and strategy-proofness. In order to establish consistency, let $S, T \in \mathcal{P}$ with $S \subseteq T$ and $e=(R, \Omega) \in \mathcal{E}^{T}$. We have to show that for the reduced economy $\tilde{e}=\left(R_{S}, \sum_{S} \varphi_{i}(e)\right)$ it follows that $\varphi(e)_{S}=\varphi(\tilde{e})$. We distinguigh the following two cases:

(a) $f_{\pi, g, \dot{\kappa}}^{T}(e) \in S$. Then, for all $i \notin S, \varphi_{i}(e)=0$ and $\tilde{e}=\left(R_{S}, \Omega\right)$. Since $\left\{f_{\pi_{+}, q_{, \pi}}^{N}\right\}_{N \in p}$ is a consistent collection of choice functions it is based on $\pi_{+}, \pi_{-} \in \mathrm{II}^{\mathrm{P}^{\mathrm{P}}}$, and a consistent collection of choice functions $\mathcal{G}=\left\{g^{N} \in G^{N}\right\}_{N \in \mathcal{P}}$. Hence, $f_{\pi, g, k}^{T}(e)=f_{\pi, g, k}^{S}(e)$ and $\varphi(e)_{S}=\varphi(\bar{e})$.

(b) $f_{\pi, g, k}^{T}(e) \notin S$. Hence, for all $i \in S, \varphi_{i}(e)=0$. So, $\sum_{S} \varphi_{i}(e)=0$ and $\ddot{e}=\left(R_{S}, 0\right)$. Thus, for all $i \in S, \varphi_{i}(\tilde{e})=0$ and $\varphi(e)_{S}=\varphi(\tilde{e})$.

Cases (a) and (b) together prove the consistency of $\varphi$.

To prove the remaining part of the theorem let $\varphi$ satisfy the properties listed in the theorem. By Lemma 3.3 it follows that $\varphi$ satisfies replacement-domination. Then, by Corollary 3.1, for each $N \in \mathcal{P}$ and $\Omega \in \mathbb{R}_{+}$there exist $\pi_{N} \in \Pi^{N}, g_{N} \in G^{N}$, and $k_{N} \in N$ such that for all $e=(R, \Omega) \in \mathcal{E}^{N}$,

$$
\varphi(e)=\Omega \chi^{f_{\pi_{N}, g_{N}, k_{N}}^{M}(e)}
$$


Definition of $\pi_{+} \in \Pi^{\$}$.

Consider the whole set of potential agents $\mathbb{P}^{P}$. Then, by consistency, $\pi_{+}:=\pi_{\mathbb{P}+} \in \Pi^{\mathbb{P}}$ is such that for all $N \subseteq \mathbb{P}$ and all $e=(R, \Omega) \in \mathcal{E}^{N}$ with $N_{+}(e) \neq \emptyset$, $\arg \min \left\{\pi_{+}(i) \mid i \in\right.$ $\left.N_{+}(e)\right\}=\arg \min \left\{\pi_{N}(i) \mid i \in N_{+}(e)\right\}$.

Definition of $\mathcal{G}=\left\{g^{N} \in G^{N}\right\}_{N \in \mathcal{P}} \in \mathcal{C}^{\mathbb{P}}$.

Consider the whole set of potential agents $\mathbb{P}$ and the collection of choice functions $\mathcal{G}_{\mathbb{P}}=\left\{g^{N} \in G^{N}\right\}_{N \subseteq \mathbb{P}}$. The collection of choice functions $\mathcal{G}=\left\{\tilde{g}^{N} \in G^{N}\right\}_{N \subseteq \mathbb{P}}$ is defined as follows For all $N \subseteq \mathbb{P}$ and all $e=(R, \Omega) \in \mathcal{E}^{N}, \tilde{g}^{N}(e)=g^{N}(e)$ if $N_{+}(e)=\emptyset$ and $N_{0}(e) \neq \emptyset$ and $\tilde{g}^{N}(e)=\min \{i \mid i \in N\}$ otherwise. By construction and consistency, it follows easily that $\mathcal{G}$ is a consistent subcollection of choice functions.

Definition of $\pi_{-} \in \Pi^{\mathbb{P}}$.

Next, define $\pi_{-} \in \Pi^{\mathbb{P}}$ as follows. Let $\pi_{-}\left(k_{\mathbb{P}}\right)=1$ and $\mathbb{P}^{1}:=\mathbb{P} \backslash\left\{k_{\mathbb{P}}\right\}$. Next, let $\pi_{-}\left(k_{\mathbb{p} 1}\right)=2$ and $\mathbb{P}^{2}:=\mathbb{P} \backslash\left\{k_{\mathbb{P}^{1}}\right\}$. Let $\pi_{-}\left(k_{\mathbb{P} 2}\right)=3$ etc. It is clear, how the linear order $\pi_{\mathbb{P}-} \in \Pi^{\mathbb{P}}$ is constructed step by step. Furthermore, by consistency, $\pi_{-} \in \Pi^{\mathbb{P}}$ is such that for all $N \subseteq \mathbb{P}$ and all $e=(R, \Omega) \in \mathcal{E}^{N}$ with $N_{-}(e)=N$, arg $\min \left\{\pi_{-}(i) \mid i \in\right.$ $\left.N_{+}(e)\right\}=k_{N}$.

Hence, for each $\Omega \in \mathbb{R}_{+}$, there exist $\pi_{+}, \pi_{-} \in \Pi^{\mathbb{P}}$ and $\mathcal{G}=\left\{\tilde{g}^{N} \in G^{N}\right\}_{N \subseteq \mathbb{P}}$ such that for all $N \subseteq \mathbb{P}$ and all $e=(R, \Omega) \in \mathcal{E}^{N}, \varphi(e)=\Omega \chi^{f_{\pi_{+}, \varphi_{1} \pi_{-}(e)}{ }^{(e)}}$

Finally, we discuss population-monotonicity: if a group of agents leaves the economy, then all remaining agents are affected in the same direction (see also Section 2.2, page 24).

Population-monotonicity: For all $N, M \in \mathcal{P}$, all $e=(R, \Omega) \in \mathcal{E}^{N}$, and all $\bar{e}=(\bar{R}, \bar{\Omega}) \in \mathcal{E}^{N}$, if $M \subseteq N$ and $R_{M}=\bar{R}$, then either [for all $i \in M, \varphi_{i}(e) R_{i} \varphi_{i}(\bar{e})$ ] or [for all $\left.i \in M, \varphi_{i}(\bar{e}) R_{i} \varphi_{i}(e)\right]$.

We show that a rule $\varphi$ that satisfies Pareto optimality, strategy-proofness, and population-monotonicity assigns the whole endowment to a single agent.

Let $\mathcal{B}_{\varphi}=\left\{e=(R, \Omega) \in \mathcal{E}^{N} \mid N \in \mathcal{P}\right.$ and for all $\left.i \in N, \varphi_{i}(e) \neq \Omega \chi^{i}\right\}$ be the set of economies that yield a broken allocation under $\varphi$, i.e., a feasible allocation where no agent obtains the whole endowment.

Lemma 3.4 Let $\varphi$ be a rule that satisfies Pareto optimality, strategy-proofness, and population-monotonicity. Then, $\mathcal{B}_{\varphi}=\emptyset$.

Proof. Let $\varphi$ satisfy the properties listed in the lemma and suppose by contradiction that $\mathcal{B}_{\varphi} \neq \emptyset$.

\footnotetext{
${ }^{12}$ Note, that for an infinite set, of potential agents represented by the natural numbers $\mathbb{N}$, the representation of the rules by means of permutations is not sufficient. However, by changing the notation and by introducing binary relations to describe the selection functions, the result extends to this infnite population setting. To be more specific, one can show that the choice rule that is induced by the rule in Case $1\left(N_{+}(e) \neq \emptyset\right)$ satisfies the weak axiom of revealed preferences (see Mas-Colell, Whinstion, and Green, 1995). Then, it follows that there exists a biriary relation that rationalizes this clioice rule. A similar argument holds for the choice rule that is induced by the rule in Case $3\left(N_{+}(e) \neq D\right)$
} 
Since $\mathcal{B}_{\varphi} \neq \emptyset$, there exist $N \in \mathcal{P}$ and $e=(R, \Omega)$ such that for all $i \in N, \varphi_{i}(e) \neq \Omega x^{i}$. Hence, there exist $i, j \in N$ such that $\varphi_{i}(e) \neq 0$ and $\varphi_{j}(e) \neq 0$. By Lemma 3.1, either (a) $\varphi_{i}(e) P_{i} 0$ and $\varphi_{j}(e) P_{j} 0$ or (b) $\varphi_{i}(e) P_{i} \Omega$ and $\varphi_{j}(e) P_{j} \Omega$.

Now, let $M=\{i, j\}$ and consider $\bar{e}=\left(R_{M}, \Omega\right) \in \mathcal{E}^{M}$. By population-monotonicity, either $\left[\varphi_{i}(e) R_{i} \varphi_{i}(\bar{e})\right.$ and $\left.\varphi_{j}(e) R_{j} \varphi_{j}(\bar{e})\right]$ or $\left[\varphi_{i}(\bar{e}) R_{i} \varphi_{i}(e)\right.$ and $\left.\varphi_{j}(\bar{e}) R_{j} \varphi_{j}(e)\right]$.

Since $M$ is a two-agent economy, by Corollary 3.2 it follows that either $\left[\varphi_{i}(\bar{e})=\Omega\right.$ and $\left.\varphi_{j}(\vec{e})=0\right]$ or $\left[\varphi_{i}(\bar{e})=0\right.$ and $\left.\varphi_{j}(\bar{e})=\Omega\right]$. This is in contradiction to (a) and (b).

Now, using the methods of proof developed in Theorem 3.1, Corollary 3.1, and Theorem 3.2, we can derive a characterization of all Pareto optimal, strategy-proof, and population-monotonic rules.

The proof of the following characterization is very similar to the proofs of Theorems 3.1 and 3.2. Therefore we omit it.

Theorem 3.3 A rule $\varphi$ satisfies Pareto optimality, strategy-proofness, and populationmonotonicity if and only if for each $\Omega \in \mathbb{R}_{+}$there exist $\pi_{+}, \pi_{-} \in \Pi^{\mathbb{P}}$ and $\mathcal{G}=\left\{g^{N} \in\right.$ $\left.G^{N}\right\}_{N \in \mathcal{P}}$ such that for all $N \in \mathcal{P}$ and all $e=(R, \Omega) \in \mathcal{E}^{N}$,

$$
\varphi(e)=\Omega \chi^{f_{\pi_{+}, \mathscr{S}, \pi_{-}}(e)} .
$$

Note that the only difference between the class of rules described in Theorem 3.3 and 3.2 is that the rules described in Theorem 3.2 are based on collections of choice functions that are in addition consistent. Hence, the class of rules that satisfy Pareto optimality, strategy-proofness, and population-monotonicity is a strict subset of the class of rules that satisfy Pareto optimality, strategy-proofness, and consistency. Furthermore, the class of rules that satisfy Pareto optimality, strategy-proofness, and population-monotonicity strictly contains the class of rules that satisfy Pareto optimality, strategy-proofness, and replacement-domination (see Corollary 3.1).

In the next example, we describe a rule that satisfies all properties we considered until now.

Example 3.2 The following rule $\varphi^{\mathrm{min}}$ satisfies Pareto optimality, strategy-proofness, replacement-domination, consistency, and population-monotonicity. For all $N \in \mathcal{P}$ and all $e=(R, \Omega) \in \mathcal{E}^{N}$ :

Case 1: If $N_{+}(e) \neq \emptyset$, then $\varphi^{\min }(e)=\Omega \chi^{\min \left\{i \mid i \in N_{+}(e)\right\}}$.

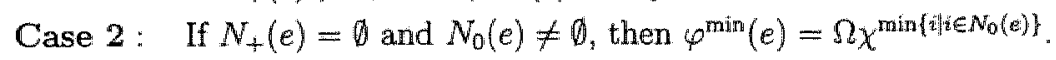

Case 3: If $N=N_{-}(e)$, then $\varphi^{\min }(e)=\Omega \chi^{\min \left\{a \mid i \in N_{-}(e)\right\}}$.

By id $\in \Pi^{\mathbb{P}}$ we denote the identity permutation defined by $\operatorname{id}(i)=i$ for all $i \in \mathbb{P}$. Let $\pi_{+}=\pi_{-}=$id $\in \Pi^{\mathbb{P}}$ and define $g_{\min }^{N} \in G^{N}$ such that for all $N \in \mathcal{P}$ and all $e=$ $(R, \Omega) \in \mathcal{E}^{N}, g_{\min }^{N}(e)=\min \left\{i \mid i \in N_{0}(e)\right\}$ if $N_{0}(e) \neq \emptyset$ and $g_{\min }^{N}(e)=\min \{\dot{i} \mid i \in N\}$ otherwise. Then, $\mathcal{G}^{\prime}:=\left\{g_{\min }^{N} \in G^{N}\right\}_{N \in \mathcal{P}}$ is a consistent collection of choice functions. Now choosing for all $\Omega \in \mathbb{R}_{+}$the consistent collection of selections $\left\{f_{\text {id, } \mathcal{G}^{\prime}, \text { id }}^{N}\right\}_{N \in \mathcal{P}}$, yields for all $N \in \mathcal{P}$ and all $e=(R, \Omega) \in \mathcal{E}^{N}, \varphi^{\min }(e)=\Omega \chi^{f_{\text {idd } G^{\prime}, \text { id }}^{(e)} \text {. }}$ 
The choice functions $g_{\mathrm{min}}^{N}$ as defined in Example 3.2 always select agents according to the identity permutation. We say that a collection of choice functions $\mathcal{G}=\left\{g^{N} \in\right.$ $\left.G^{N}\right\}_{N \in \mathcal{P}}$ is based on a linear order $\pi \in \mathbb{\Pi}^{\mathcal{P}}$ if for all $N \in \mathcal{P}$ and all $e=(R, \Omega) \in \mathcal{E}^{N}$, $g^{N}(e)=\arg \min \left\{\pi(i) \mid i \in N_{0}(e)\right\}$ if $N_{0}(e) \neq \emptyset$. It is easy to show that all collections of choice functions $\mathcal{G}=\left\{g^{N} \in G^{N}\right\}_{N \in \mathcal{P}}$ that are based on a linear order $\pi \in \Pi^{\mathcal{P}}$ are consistent. However, as the following example shows, the class of consistent collections of choice functions strictly contains the class of choice functions that are based on a linear order $\pi \in \Pi^{\mathbb{P}}$.

Example 3.3 We define the class of collections $\mathcal{G}^{\prime \prime}=\left\{\bar{g}^{N} \in G^{N}\right\}_{N \in \mathcal{P}}$ as follows. For all $N \in \mathcal{P}$ and all $e=(R, \Omega) \in \mathcal{E}^{N}, \bar{g}^{N}(e)=\min \left\{i \mid i \in \arg \min \left\{d\left(R_{i}\right) \mid i \in N_{0}(e)\right\}\right\}$ if $N_{0}(e) \neq \emptyset$ and $\bar{g}^{N}(e)=\min \{i \mid i \in N\}$ otherwise. Note that now we only use the identity permutation to break the tie if there are several agents in $N_{0}(e)$. with the same smallest dip.

Remark 3.2 By going over the proofs the reader may verify that we do not fully exploit the "richness" of the domain of single-dipped preferences. The results remain true for any sub-domain $\mathcal{D}^{\prime} \subseteq \mathcal{D}$ of preferences such that for all $x \in(0, \Omega)$, there exist $\tilde{R}, \tilde{R} \in \mathcal{D}^{\prime}$ with $0 \bar{P} \Omega \bar{P} x$ and $\Omega \tilde{P} 0 \tilde{P} x$.

\subsection{Independence, compatibility, and incompatibil- ity of properties}

First we show that the characterizations of Theorem 3.1, Corollary 3.1, Theorems 3.2, and 3.3 are tight, i.e., dropping any of the properties yields alternative rules.

Example 3.4 The following rule $\bar{\varphi}$ satisfies strategy-proofness, replacement-domination,

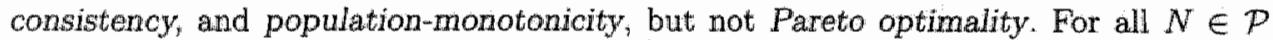
and all $e=(R, \Omega) \in \mathcal{E}^{N}, \tilde{\varphi}(e)=\Omega \chi^{\min (i|i| i \in N}$.

Example 3.5 The following rule $\hat{\varphi}$ satisfies Pareto optimality, replacement-domination, consistency, and population-monotonicity, but not strategy-proofness. For all $N \in \mathcal{P}$ and all $e=(R, \Omega) \in \mathcal{E}^{N}$ such that $N_{+}(e) \neq \emptyset$, let $N_{+}^{1}(e)=\left\{i \in N_{+}(e) \mid d\left(R_{i}\right)=0\right\}$ and $N_{+}^{2}(e)=\left\{i \in N_{+}(e) \mid d\left(R_{i}\right) \neq 0\right\}$. Then, for all $N \in \mathcal{P}$ and all $e=(R, \Omega) \in \mathcal{E}^{N}$, we define $\hat{\varphi}(e)$ as follows. If $N_{+}^{1}(e) \neq \emptyset$, then $\hat{\varphi}(e)=\Omega \chi^{\min \left\{i \mid i \in N_{+}^{1}(e)\right\}}$, if $N_{+}^{1}(e)=\emptyset$ and $N_{+}^{2}(e) \neq \emptyset$, then $\hat{\varphi}(e)=\Omega \chi^{\min \left\{a \mid i \in N_{+}^{2}(e\}\right\}}$, and $\hat{\varphi}(e)=\varphi^{\min }(e)$ otherwise.

Example 3.6 The following rule $\bar{\varphi}$ satisfies Pareto optimality, strategy-proofness, but none of the following properties replacement-domination, consistency, and populationmonotonicity. For $T=\{1,2,3\}$ and $e=(R, 1) \in \mathcal{E}^{T}$, define $\tilde{\varphi}(e)$ as follows. If $1 P_{3} 0$, then

$$
\tilde{\varphi}(e)= \begin{cases}\left(\frac{1}{2}, \frac{1}{2}, 0\right) & \text { if } 1 P_{1} \frac{1}{2} P_{1} 0 \text { and } 1 P_{2} \frac{1}{2} P_{2} 0, \\ (0,0,1) & \text { otherwise. }\end{cases}
$$

For all remaining $N \in \mathcal{P}$ and $e=(R, \Omega) \in \mathcal{E}^{N}, \tilde{\varphi}(e)=\varphi^{\min }(e)$. 
In the remainder of the section, we explore the compatibility of Pareto optimality with other well-known properties for rules we introduced in Chapter 2.

In the model of fair allocation with single-peaked preferences, many rules satisfy peak-onliness: the rule only depend on the peaks instead of the complete profile of preferences (see Section 2.2, page 22). Peak-onliness not only simplifies the application of a rule to a given problem, it also incorporates a notion of nonmanipulability with respect to intensities of the preferences or the "shape" of the preferences. The natural counterpart of peak-onliness in this model is dip-onliness. For a profile $R=\left(R_{i}\right)_{i \in N} \in \mathcal{D}^{N}$, by $d(R)=\left(d\left(R_{i}\right)\right)_{i \in N}$ we denote the vector of dips.

Dip-onliness: $\quad$ For all $N \in \mathcal{P}$, all $e=(R, \Omega) \in \mathcal{E}^{N}$, and all $\bar{e}=(\bar{R}, \Omega) \in \mathcal{E}^{N}$, if $d(R)=d(\bar{R})$, then $\varphi(e)=\varphi(\bar{e})$.

By Lemma 3.1 it follows easily that Pareto optimality of an allocation does not only depend on the agents' dips but on the whole preference profile; loosely speaking, Pareto optimality depends on the shapes and intensities of the individual preferences. Thus, dip-onliness is not compatible with Pareto optimality.

The next property we consider represents a standard notion of fairness: individual rationality from equal division requires that each agent (weakly) prefers his share to the "equal division share" of the economy (see Section 2.2, page 27).

Individual rationality from equal division: For all $N \in \mathcal{P}$, all $e=(R, \Omega) \in \mathcal{E}^{N}$, and all $i \in N, \varphi_{i}(e) R_{i} \frac{\Omega}{|N|}$.

Note that for each economy $e \in \mathcal{E}^{N}$ the equal division allocation $z=\left(\frac{\Omega}{|N|}, \ldots, \frac{\Omega}{|N|}\right)$ is feasible. If $z$ is not Pareto optimal, then proceeding from $z$ we can easily construct an allocation $y$ that Pareto dominates $z$ and that is Pareto optimal. Hence, Pareto optimal and individually rational from equal division allocations always exist.

Next, we consider the compatibility of Pareto optimality with the properties of fairness anonymity, no-envy, and equal treatment of equals.

Anonymity requires that the agents' shares do not depend on their names (see Section 2.2, page 22). No-envy states that no agent strictly prefers the share of another agent to his own share (see Section 2.2, page 22). Equal treatment of equals, a weakening of no-envy, requires that if two agents have the same preference relation, then each of them is indifferent between his share and the other agent's share (see Section 2.2, page 23).

Let $\pi \in \mathbb{\Pi}^{N}$ be a permutation on $N$ and $R \in \mathcal{D}^{N}$. Then, $R_{\pi}=\left(R_{\pi(i)}\right)_{i \in N}$ and $e_{\pi}=\left(R_{\pi}, \Omega\right)$.

Anonymity: For all $N \in \mathcal{P}$, all $e=(R, \Omega) \in \mathcal{E}^{N}$, all $\pi \in \Pi^{N}$, and all $i \in N$, $\varphi_{i}\left(e_{\pi}\right)=\varphi_{\pi(i)}(e)$. 
No-envy: For all $N \in \mathcal{P}$, all $e=(R, \Omega) \in \mathcal{E}^{N}$, and all $i, j \in N, \varphi_{i}(e) R_{i} \varphi_{j}(e)$.

Equal treatment of equals: For all $N \in \mathcal{P}$, all $e=(R, \Omega) \in \mathcal{E}^{N}$, and all $i, j \in N$, if $R_{i}=R_{j}$, then $\varphi_{i}(e) I_{i} \varphi_{j}(e)$.

By the next example, we show that Pareto optimality is generally not compatible with the properties introduced above.

Example 3.7 Let $N=\{1,2\}, \Omega=10$, and $e=(R, \Omega) \in \mathcal{E}^{N}$ be such that $R_{1}=R_{2}$ and $d\left(R_{1}\right)=7,0 I_{1} 9$, and $2 I_{1} 8$. Suppose the rule $\varphi$ satisfies Pareto optimality. Then, by Lemma 3.1, for $i=1,2$, either $\varphi_{i}(e) \in\{0,10\}$ or $\varphi_{i}(e) \in(9,10)$. Since $\varphi_{2}(e)=$ $10-\varphi_{1}(e)$, it follows, by feasibility, that $\varphi(e) \in\{(10,0),(0,10)\}$ If $\varphi$ is anonymous, then $\varphi(e)=(5,5)$. If $\varphi$ satisfies no-envy or equal treatment of equals, then $\varphi(e) \in$ $\{(5,5),(2,8),(8,2)\}$. However, none of these allocations is Pareto optimal.

Since replication-invariance (see Section 2.2, page 25) implies anonymity, Example 3.7 also shows the incompatibility of Pareto optimality and replication-invariance.

The next example shows that Pareto optimality and continuity with respect to preferences are not compatible. In the example we apply continuity with respect to preferences in an informal way: continuous changes of the preferences imply continuous changes in the allocations assigned by the rule. For the problem of fair allocation when preferences are single-peaked, continuity with respect to preferences was introduced by Sprumont (1991). This definition can easily be adjusted for rules when preferences are single-dipped.

Example 3.8 Let $N=\{1,2\}$ and $e=\left(\left(R_{1}, R_{2}\right), \Omega\right) \in \mathcal{E}^{N}$ be such that $0 I_{1} \Omega$ and $\Omega P_{2} 0$. Suppose the rule $\varphi$ satisfies Pareto optimality. Then, $\varphi\left(\left(R_{1}, R_{2}\right), \Omega\right)=(0, \Omega)$. Now, change $R_{2}$ continuously into $R_{1}$ in such a way that in these changes agent 1 still prefers $\Omega$ to 0 . Continuity with respect to preferences implies (in the limit) $\varphi\left(\left(R_{1}, R_{1}\right), \Omega\right)=(0, \Omega)$. Next, consider $\tilde{e}=\left(\left(R_{2}, R_{1}\right), \Omega\right) \in \mathcal{E}^{N}$. Then, by the same argument it follows that $\varphi\left(\left(R_{2}, R_{1}\right), \Omega\right)=(\Omega, 0)$ and (in the limit) $\varphi\left(\left(R_{1}, R_{1}\right), \Omega\right)=(\Omega, 0)$. This is in contradiction to $\varphi\left(\left(R_{1}, R_{1}\right), \Omega\right)=(0, \Omega)$.

We conclude this section with a compatibility result concerning the property of converse consistency (see Section 2.2, page 28). As already explained in Chapter 2, converse consistency determines the desirability of an allocation on the basis of the desirability of its restrictions to all two-agent reduced economies.

Converse consistency: For all $N \in \mathcal{P}$, all $e=(R, \Omega) \in \mathcal{E}^{N^{N}}$, and all feasible allocations $x \in \mathbb{R}_{+}^{N}$, if for all $M \subseteq N$ with $|M|=2, \varphi\left(R_{M}, \sum_{M} x_{i}\right)=x_{M}$, then $\varphi(e)=x$.

By Theorem 3.2 it is easy to prove that all rules satisfying Pareto optimality, strategy-proofness, and consistency are conversely consistent. 
Since in Corollary 3.1 for each set of agents, an arbitrary linear order, choice function, and agent can be selected, it is easy to show that not all rules satisfying Pareto optimality, strategy-proofness, and replacement-domination are conversely consistent.

As the following example shows, a Pareto optimal, strategy-proof, and conversely consistent rule does not necessarily always assign the whole amount to a single agent.

Example 3.9 The following allocation rule $\varphi^{c}$ satisfies Pareto optimality, strategyproofness, and converse consistency. Let $T_{1}=\{1,2\}, T_{2}=\{2,3\}$, and $T_{3}=\{1,3\}$. For all $e_{1}=\left(R^{1}, \Omega\right) \in \mathcal{E}^{T_{1}}$ such that $N_{+}\left(e_{1}\right)=T_{1}$, let $\varphi_{1}^{c c}\left(e_{1}\right)=\Omega$, for all $e_{2}=\left(R^{2}, \Omega\right) \in \mathcal{E}^{T_{2}}$ such that $N_{+}\left(e_{2}\right)=T_{2}$, let $\varphi_{2}^{c c}\left(e_{2}\right)=\Omega$, and for all $e_{3}=\left(R^{3}, \Omega\right) \in \mathcal{E}^{T_{3}}$ such that $N_{+}\left(e_{3}\right)=T_{3}$, let $\varphi_{3}^{c c}\left(e_{3}\right)=\Omega$. For all remaining economies $e=(R, \Omega) \in \mathcal{E}^{N}, N \in \mathcal{P}$, let $\varphi^{c c}(e)=\tilde{\varphi}(e)$, where $\tilde{\varphi}$ is defined in Example 3.6.

The proof that $\varphi^{c c}$ satisfies converse consistency is easy. It is based on the following two cases.

Case 1: The allocation assigned for $N \in \mathcal{P}$ with $|N|>2$ and $e \in \mathcal{E}^{N}$ is consistent with the choice for all two-agent economies (if $\{1,2,3\} \cap N \neq\{1,2,3\}$ ).

Case 2: There exists no allocation that satisfies the hypothesis of converse consistency, namely, that the allocation for each reduced two-agent economy equals the restriction of the allocation to this set (this follows from the "cycle" we constructed for the two-agent subsets of $\{1,2,3\})$. 


\section{Part II}

\section{Fair reallocation}




\section{Chapter 4}

\section{The uniform reallocation rule ${ }^{1}$}

We consider the problem of reallocating the individual endowments of some perfectly divisible commodity among a group of agents with single-peaked preferences. Such reallocation problems may occur when we are concerned with allocation problems, as described in Chapter 2, where preferences might change over time. Consider the following example. A task or a certain amount of work has to be divided among a group of workers. Assume that they are rewarded in proportion to their shares. Then, preferences over individual shares are single-peaked: each worker has a most preferred share, his peak, below which and above which his welfare is decreasing. Suppose now that we solved the allocation problem. If after a certain period of time preferences have changed, then it might be the case that the present allocation can be improved upon by reallocation. In other settings individual endowments are directly given, e.g., in fixedprice exchange economies (see Benassy 1982), or can be interpreted as natural claims or priorities, e.g., investments, as described by Barberà, Jackson, and Neme (1997).

There is a wide literature exploring the situation where the problem is reduced to the allocation of a total or social endowment. As described in Sprumont (1991), rationing in a two-good economy in which prices are in disequilibrium can be interpreted as such an allocation problem with total endowment. For this class of problems the preeminently featured allocation rule is the uniform rule (see Chapter 2). Sprumont (1991) started the axiomatic analysis of this class of problems and gave the first characterizations of the uniform rule (see Theorems 2.1 and 2.2). Since then, a variety of axiomatic studies, which also led to this rule, have been published. We refer the reader to the overview of the most important results given in Chapter 2 .

In this chapter we are concerned with the axiomatic analysis of reallocation rules and particularly with the so-called uniform reallocation rule. Here, we explore to what extent the results as achieved for the allocation model can be generalized to the more general reallocation model. First, we extend the model and the properties we introduced in Chapter 2 to the reallocation setting. After exploring the compatibilities and the incompatibilities of this properties, it turns out that, again, a single rule comes out repeatedly as the unique solution satisfying certain combinations of desirable properties:

\footnotetext{
${ }^{1}$ This chapter is mainly based on Klaus, Peters, and Storcken (1995a,b, 1997b), Klaws (1997b,d).
} 
the uniform reallocation rulle.

This chapter is organized as follows. First, in Section 4.1, we give an overview, or more precisely a preview, of the results presented in this chapter. Furthermore, we discuss the parallels and the differences to former allocation results. In Section 4.2 we introduce the reallocation model and the uniform reallocation rule. In Sections $4.3-4.5$ we introduce and explore properties for reallocation rules. Furthermore, we derive several characterizations of the uniform reallocation rule and discuss the relation to similar allocation results. We show the independence of the properties in the characterizations in Section 4.6. A discussion of the sensitivity of the results to variations in the model parameters is postponed to Chapter 5.

\subsection{A preview of the results}

In Section 4.2 we first introduce the reallocation model. In contrast to the allocation problem, where a social endowment has to be divided among a group of agents, the individual endowments have to be reallocated among them. A reallocation rule assigns to each reallocation economy that is determined by a group of agents, individual endowments, and their preference relations a "reallocation" of the resources among the agents of the group. Similar to the allocation model, a central property of reallocation rules is Pareto optimality. As examples for Pareto optimal reallocation rules, we introduce the proportional and the uniform reallocation rule (see Section 4.2 for the exact definitions).

In Section 4.3.1, similar to the allocation model, we start the analysis of reallocation rules with the well-known properties strategy-proofness and no-envy. As usual, strategy-proofness requires that no agent ever gains by lying about his preference relation. No-envy for reallocation rules is formalized in terms of allotment changes rather than in terms of net allotments: no agent strictly prefers another agent's allotment change, or the part of this allotment change that is "feasible" for him, to his own allotment change. It turns out that, similar to the uniform allocation rule (see Theorem 2.2 ) the uniform reallocation rule is the only rule that satisfies Pareto optimality, no-envy, and strategy-proofness (see Theorem 4.1).

Next, we consider two weakenings of no-envy" weak no-envy requires that no agent strictly prefers another agent's allotment change that is feasible for him, to his own allotment change, and equal treatment of equals requires that if two agents have the same demand, then each of them is indifferent between receiving his allotment change and receiving the part of the other agents' allotment change that is feasible for him. In Corollaries 4.1 and 4.2 we prove that if a reallocation rule satisfies Pareto optimality, strategy-proofness, and either weak no-envy or equal treatment of equals, then this rule assigns the uniform reallocation to economies with excess demand.

In Section 4.3.2, we study endowment strategy-proofness. If individual endowments are private information, then it might happen that agents manipulate the outcome by

\footnotetext{
${ }^{2}$ Here, the feasible part of an allotment change yields either a nonnegative allotment or the allotment zero.
} 
only reporting, or exhibiting, a smaller part of their individual endowments. Reallocation rules where no agent can ever profit from withholding a part of his endowment are called endowment strategy-proof. First; we prove that the uniform reallocation rule satisfies endowment strategy-proofness (see Lemma 4.7). Furthermore, we prove that if a reallocation rule satisfies Pareto optimality, endowment strategy-proofness, and weak no-envy, then this rule assigns the uniform reallocation to economies with excess demand (see Corollary 4.4).

The first property we study in Section 4.3 .3 is demand-onliness: the agents' allotment changes only depend on the agents' demands. As an extension of Ching's characterization of the uniform allocation rule by Pareto optimality, strategy-proofness, and equal treatment of equals (see Theorem 2.4) we prove that the uniform reallocation rule is the only rule that satisfies Pareto optimality, strategy-proofness; equal treatment of equals, and demand-onliness (see Corollary 4:5). Then, it follows easily that the uniform reallocation rule is the only rule that satisfies Pareto optimality, strategyproofness, anonymity, and demand-onliness (see Corollary 4.6). This characterization can be interpreted as an extension of one of Sprumont's characterization of the uniform allocation rule (see Theorem 2.1) to the reallocation model.

In the remainder of Section 4.3.3, we study a strengthening of demand-onliness, called reversibility. Reversibility links the allotment changes of excess demand economies to the allotment changes of certain excess supply economies. To be more precise, consider a situation with demanders, having their peaks above their initial endowments, and suppliers, having their peaks below their initial endowments. Now this situation is reversed by turning demanders into suppliers whose supplies are equal to their former demands and suppliers into demanders in a similar way. Then, reversibility requires that the allotment changes of the latter problem are opposite to those of the former. In Theorem 4.2 we show that the uniform reallocation rule is the only rule that satisfies Pareto optimality, equal treatment of equals, endowment strategy-proofness, and reversibility.

The last property we introduce in Section 4.3 .3 is a weakening of demandwonliness that is well-known from the allocation model. Peak-onliness, loosely speaking, requires that the reallocation rule only depends on the peaks instead of the complete preference profile. As an extension of Thomson's characterization of the uniform allocation rule by Pareto optimality, no-envy, and peak-onliness (see Theorem 2.3) we prove that the uniform reallocation rule is the only rule that satisfies Pareto optimality, no-envy, and peak-onliness (see Theorem 4.3).

Section 4.4 is devoted to so-called solidarity properties. By "solidarity" we mean that if certain changes in the model parameters (e.g. the population, one agent's preference relation, or one agent's individual endowment) occur, then agents that are not responsible for this change should all (weakly) lose together or (weakly) gain together. Similar to the allocation model the solidarity properties are only compatible with other desirable properties (e.g., Pareto optimality and no-envy) if they apply to changes that do not turn an economy with excess demand into an economy with excess supply, or vice versa. Therefore we concentrate on this "one-sided" version of the solidarity properties.

The first solidarity property we study is one-sided population-monotonicity. In the 
reallocation model this property describes the impact of merging two economies. We show that the uniform reallocation rule is the only rule that satisfies Pareto optimality, weak no-envy, and one-sided population-monotonicity (see Theorem 4.4). This characterization can be interpreted as an extension of the following characterization (see Theorem 2.6) to the reallocation model: the uniform allocation rule is the only rule that satisfies Pareto optimality, no-envy, one-sided population-monotonicity, and replication-invariance, i.e., if an economy is replicated, then also the assigned allocation must be replicated.

A further solidarity property, introduced by Thomson for the allocation model is one-sided replacement-domination: if one agent changes his preference relation, then either all remaining agents (weakly) lose or all (weakly) gain. It turns out that in the reallocation model as well as in the allocation model the uniform rule is the only rule that satisfies Pareto optimality, no-envy, one-sided replacement-domination, and replication-invariance (see Theorem 4.5 for the uniform reallocation rule and Theorem 2.7 for the uniform allocation rule characterization).

The last solidarity type property we consider is one-sided endowment-monotonicity. It describes the change of the solution if certain unilateral changes in the individual endowments occur: if the change of one agent's individual endowment is one-sided, then either all agents (weakly) lose or all (weakly) gain. A stronger notion of one-sided endowment-monotonicity is the following strong one-sided endowment-monotonicity. If in case of excess demand an agent's individual endowment decreases, then no agent is made better off in the reallocation that follows this one-sided change. Similarly, if in case of excess supply an agent's individual endowment increases, then no agent is made better off in the reallocation that follows this one-sided change.

The two endowment-monotonicity properties we mentioned above can be seen as extensions of the one-sided resource-monotonicity as introduced by Thomson for the allocation model. By Theorem 2.5 , the uniform allocation rule is the only rule that satisfies Pareto optimality, no-envy, and one-sided resource-monotonicity for a restricted domain of single-peaked, continuous preferences. For the reallocation case, a similar result (see Theorem 4.6) can be deduced: the uniform reallocation rule is the only rule that satisfies Pareto optimality, weak no-envy, and one-sided endowment-monotonicity. In contrast to the allocation model, the proof of Theorem 4.6 is based on different arguments than the proof of Theorem 2.5. Furthermore, Theorem 4.6 remains valid for the whole domain of single-peaked preferences. After showing that individual rationality and strong one-sided endowment-monotonicity imply Pareto optimality, it follows easily that the uniform reallocation rule is the only rule that satisfies individual rationality, strong one-sided endowment-monotonicity, and weak no-envy (see Corollary $4.9)$.

The next property of the uniform reallocation rule we study is bilateral consistency. In the allocation model, bilateral consistency requires the following. Consider an allocation situation where all agents except two leave with their assigned shares of the social endowment. Then, the corresponding reduced economy is formed by the remaining agents, their preference relations, and the remaining "social" endowment. If for this reduced economy the shares for the two agents are the same as before, then bilateral 
consistency is satisfied. Thomson (1994b) provides two characterizations of the uniform rule by means of Pareto optimality, bilateral consistency, and continutity with respect to the social endowment. In the first characterization (see Theorem 2.8) no-envy singles out the uniform rule. In the second characterization (see Theorem 2.9) no-envy is replaced by individual rationality from equal division: no agent, after the allocation, is worse off than in the case of equally dividing the social endowment. In a recent study, Dagan shows that under certain circumstances the continuity property can be skipped (see Theorems 2.11 and 2.12).

Now, consider a reallocation situation where all agents except two leave with their assigned shares of the good. Then, the corresponding reduced economy is formed by the remaining agents, their preference relations, and individual endowments where the leftover (supply or demand) of the departing agents is, up to domain restrictions, added equally to the original individual endowments of the remaining agents. If for this reduced economy the shares for the two agents are the same as before, then bilateral consistency is satisfied. As an extension of the results we mentioned above, we show that the uniform reallocation rule is the only rule that satisfies Pareto optimality, bilateral consistency, and either individual rationality or weak no-envy (see Theorems 4.9 and 4.10). Further characterizations of the uniform reallocation rule can be obtained by Pareto optimality, bilateral consistency and extra conditions, for instance boundedness (of the outcome) by endowments and peaks (see Theorem 4.8), or endowment strategy-proofness and the dummy property: agents who have their peak as individual endowment do not participate in the reallocation (see Theorem 4.11).

\subsection{The model}

There is an infinite population of potential agents, indexed by the natural numbers $\mathbb{N}$. Each agent $i \in \mathbb{N}$ is described in terms of an individual endowment $w_{i} \in \mathbb{R}_{+}$of an infinitely divisible commodity, and a continuous and single-peaked preference relation $R_{i}$ defined over the non-negative real numbers $\mathbb{R}_{+}$. Single-peakedness of $R_{i}$ means that there exists a point $p\left(R_{i}\right) \in \mathbb{R}_{+}$, called the peak of agent $i$, with the following property: for all $x, y \in \mathbb{R}_{+}$with $x<y \leq p\left(R_{i}\right)$ or $x>y \geq p\left(R_{i}\right)$, we have $y P_{i} x^{3}$. Each preference relation $R_{i}$ can be described in terms of the preference function $r_{i}: \mathbb{R}_{+} \rightarrow \mathbb{R}_{+} \cup\{\infty\}$ that assigns to each point $x \in \mathbb{R}_{+}$the point on the other side of $p\left(R_{i}\right)$ that is indifferent to $x$, if such a point exists, and otherwise 0 or $\infty$, depending on which side of the peak

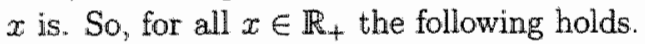

$$
\text { If } x \leq p\left(R_{i}\right) \text {, then } r_{i}(x) \geq p\left(R_{i}\right) \text { and } \begin{cases}r_{i}(x) I_{i} x & \text { if such a point } \\ r_{i}(x)=\infty & \text { exists, or }\end{cases}
$$

\footnotetext{
${ }^{3}$ As usual, $x R_{i} y$ is interpreted as " $x$ is weakly preferred to $y$ " and $x P_{i} y$ as " $x$ is sitrictly preferred to $y^{\prime \prime}$. Furthermore, $x I_{i} y$ means that agent $i$ is indifferent between $x$ and $y^{\prime}$.
} 
If $x \geq p\left(R_{i}\right)$, then $r_{i}(x) \leq p\left(R_{i}\right)$ and $\begin{cases}r_{i}(x) I_{i} x & \text { if such a point } \\ & \text { exists, or } \\ r_{i}(x)=0 & \text { otherwise. }\end{cases}$

By $\mathcal{R}$ we denote the class of all continuous, single-peaked preference relations over $\mathbb{R}_{+}$. By $\mathcal{N}$ we denote the class of non-empty and finite subsets of $\mathbb{N}$. For $N \in \mathcal{N}, \mathcal{R}^{N}$ denotes the set of (preference) profiles $R=\left(R_{i}\right)_{i \in N}$ such that for all $i \in N, R_{i} \in \mathcal{R}$. For profile $R \in \mathcal{R}^{N}, p(R)=\left(p\left(R_{i}\right)\right)_{i \in N}$ denotes the corresponding vector of peaks.

Now, an economy is formalized as follows. Let $N \in \mathcal{N}$ and $\omega=\left(\omega_{i}\right)_{i \in N} \in \mathbb{R}_{+}^{N}$ be a vector of individual endowments that have to be reallocated among the agents in $N$ with profile $R \in \mathcal{R}^{N}$. We call a pair $e=(R, \omega) \in \mathcal{R}^{N} \times \mathbb{R}_{+}^{N}$ an econamy. Let $\mathcal{E}^{N}=\mathcal{R}^{N} \times \mathbb{R}_{+}^{N}$ be the class of all economies. A feasible allacation for $e=(R, \omega) \in \mathcal{E}^{N}$, or a reallocation for short, is a vector $x \in \mathbb{R}_{++}^{N}$ such that $\sum_{N} x_{i}=\sum_{N} \omega_{i}$. A reallocation rule $\varphi$, or a rule for short, is a function that assigns to every $N \in \mathcal{N}$ and every $e=(R, \omega) \in \mathcal{E}^{N}$ a reallocation, denoted by $\varphi(e)$.

Let $N \in \mathcal{N}$ and $e=(R, \omega) \in \mathcal{E}^{N}$. Given $i \in N$, we call $\varphi_{i}(e)$ the share or allotment of agent $i$ and $\Delta \varphi_{i}(e):=\varphi_{i}(e)-\omega_{i}$ his allotment change. Agent $i$ is satiated at $\varphi(e)$ if $p\left(R_{i}\right)=\varphi_{i}(e)$ and non-satiated at $\varphi(e)$ if $p\left(R_{i}\right) \neq \varphi_{i}(e)$.

Until now, the "reallocation model" we introduce in this chapter almost equals the "allocation model" as introduced in Chapter 2: the only difference is that we have a vector of individual endowments $\omega \in \mathbb{R}_{+}^{N}$ instead of a social endowment $\Omega \in \mathbb{R}_{+}$. In contrast with the allocation model, not only the individual preferences but also the individual endowments discriminate the agents from each other. Some of these discriminations, for example having a greater endowment than one's peak, turn out to be important in the sequel.

Let $N \in \mathcal{N}$ and $e=(R, \omega) \in \mathcal{E}^{N}$. We call agent $i \in N$ a demander if his endowment is strictly less than his peak: he "demands" $p\left(R_{i}\right)-\omega_{i}$ units of the commodity. For all $i \in N$, we denote this demand by $d_{i}(e)$ and the set of demanders by $D(e)$. We call agent $i \in N$ a supplier if his endowment is strictly greater than his peak: he wants to "supply" $\omega_{i}-p\left(R_{i}\right)$ units of the commodity. For all $i \in N$, we denote this supply by $s_{i}(e)$ and the set of suppliers by $S(e)$. We call agent $i \in N$ a non-trader if his endowment is equal to his peak: he favors no trade, i.e., $d_{i}(e)=s_{i}(e)=00^{5}$ Let $d(e):=\sum_{i \in D(e)} d_{i}(e)$ denote total demand and $s(e):=\sum_{i \in S(e)} s_{i}(e)$ total supply. Furthermore, let $z(e):=\sum_{i \in N} d_{i}(e)$ denote excess demand. The latter may be positive, zero, or negative. If it is zero, then $e$ is balanced. If it is positive, then $e$ is an economy with excess demand. If it is negative, then $e$ is an economy with excess supply. Note that for all $i \in N, d_{i}(e)=-s_{i}(e)$ and $z(e)=d(e)-s(e)$.

Note that free disposal of the commodity is not allowed.

${ }^{5}$ Our notion of demanders, suppliers, and nom-traders is somewhat different from what it is in "classical exchange economies". There, the types of the agents not only depend on their own characteristics but also on prices. However, for economies with individual endowments and single-peaked preferences derived from fixed-price exchange economies, the notions coincide. 
The first property of rules we consider is the well-known requirement of Pareto optimality: a reallocation assigned by the rule cannot be changed in such a way that no agent is worse off and some agent is better off.

Because from the context it will be clear if a property is related to an allocation or a reallocation rule, the same names will be used.

Pareto optimality: For all $N \in \mathcal{N}$ and all $e=(R, \omega) \in \mathcal{E}^{N}$, there is no reallocation $x \in \mathbb{R}_{+}^{N}$, such that for all $i \in N, x_{i} R_{i} \varphi_{i}(e)$, and for some $j \in N, x_{j} P_{j} \varphi_{j}(e)$.

Note, that Pareto optimality for reallocation rules is the same as Pareto optimality for allocation rules as discussed in Chapter 2 (see Section 2.1, page 19). Similarly as before it follows easily that a rule is Pareto optimal if and only if it is same-sided, that is: for all $N \in \mathcal{N}$ and all $e=(R, \omega) \in \mathcal{E}^{N}$, either [for all $i \in N, \varphi_{i}(e) \leq p\left(R_{i}\right)$ ], or [for all $\left.i \in N, \varphi_{i}(e) \geq p\left(R_{i}\right)\right]$.

Examples of Pareto optimal reallocations can be derived as follows.

Let $N \in \mathcal{N}$ and $e=(R, \omega) \in \mathcal{E}^{N}$ be an economy with excess demand. In this case, it is the set of demanders that are on the long side of the market. ${ }^{6}$ One can argue that it is reasonable to reward the agents on the short side of the market, i.e., suppliers and non-traders, with their peaks and to reallocate the so obtained supply Pareto optimally among the demanders. ${ }^{7}$

For $N \in \mathcal{N}$ and $e=(R, \omega) \in \mathcal{E}^{N}$ such that the economy is balanced the only Pareto optimal reallocation is the vector of peaks $p(R)$.

Let $N \in \mathcal{N}$ and $e=(R, \omega) \in \mathcal{E}^{N}$ be an economy with excess supply. In this case, it is the set of suppliers that are on the long side of the market. Now, one can argue that it is reasonable to let the suppliers Pareto optimally supply part of their endowments so that the agents on the short side of the market, i.e., demanders and non-traders, receive their peaks.

In summary, a Pareto optimal reallocation can be derived by assigning to the agents on the short side of the market their peaks and by reallocating the difference between the sum of their individual endowments and their peaks, possibly a negative amount, among the remaining agents according to a Pareto optimal allocation rule (see Chapter 2). Now, applying the notions of fairness we already considered in Chapter 2 to this (re)allocation yields the following reallocation rules.

A rule that incorporates the proportional principle, and therefore embodies Aristothe's notion of justice, is the proportional (reallocation) rule $\mathrm{Pr}^{r}$ (for the proportional allocation rule, see Section 2.1, page 19). In case of excess demand, suppliers and nontraders receive their peaks. The amount they supply is divided among the remaining agents so that each of them receives in addition to his individual endowment an amount

\footnotetext{
"We: follow Benassy"s terminology of the "short" and the "long" side of a market; Benassy (1982). page 10: "The "short" side of a market is that where the aggregate volume of desired transactions is smallest. It is thus the demand side if there is excess supply, the supply sicle if there is excess demand. The other side is the "long" side."

${ }^{7}$ Note that we consider non-traders to be on the short sidle of the market.
} 
that is proportional to his demand. If the economy is balanced, each agent receives his peak. In case of excess supply, each supplier supplies a part of his endowment that is proportional to his supply in such a way that demanders and non-traders receive their peaks.

Proportional rule $P_{r^{r}}$ : For all $N \in \mathcal{N}$, all $e=(R, \omega) \in \mathcal{E}^{N}$, and all $j \in N$,

$$
\operatorname{Pr}_{j}^{\top}(e):= \begin{cases}\min \left\{p\left(R_{j}\right)_{1} \omega_{j}+\lambda d_{j}(e)\right\} & \text { if } z(e) \geq 0 \\ \max \left\{p\left(R_{j}\right)_{3} \omega_{j}+\lambda s_{j}(e)\right\} & \text { if } z(e) \leq 0\end{cases}
$$

where $\lambda \in[0,1]$ solves $\sum_{N} \operatorname{Pr}_{i}^{r}(e)=\sum_{N} \omega_{i}$.

The principle of equal reallocation under the additional assumption of Pareto optimality is described by the uniform (reallocation) rule $U^{r}$ (for the uniform allocation rule, see Section 2.1, page 19). In case of excess demand, suppliers and non-traders receive their peaks. The amount they supply is divided among the remaining agents so that each of them receives in addition to his individual endowment an amount that is as equal as possible, with the agents' peaks as upper bound. If the economy is balanced, each agent receives his peak. In case of excess supply, the reallocation is dual to the excess demand case: all demanders and non-traders receive their peaks. The amount they demand is subtracted from the remaining agents so that each of them diminishes his individual endowment by an amount that is as equal as possible, with the agents" peaks as lower bound.

Benassy (1982), Appendix J, referred to the uniform (reallocation) rule $U^{r}$ in the slightly different setting of rationing.

Uniform rule $U^{r}$ : For all $N \in \mathcal{N}$, all $e=(R, \omega) \in \mathcal{E}^{N}$, and all $j \in N$,

$$
U_{j}^{r}(e)= \begin{cases}\min \left\{p\left(R_{j}\right)_{,} \omega_{j}+\lambda\right\} & \text { if } z(e) \geq 0 \\ \max \left\{p\left(R_{j}\right)_{,} \omega_{j}+\lambda\right\} & \text { if } z(e) \leq 0\end{cases}
$$

where $\lambda \in \mathbb{R}$ solves $\sum_{N} U_{i}^{r}(e)=\sum_{N} \omega_{i}$.

So, agents are either satiated or receive the same (maximal or minimal) allotment change. In fact, combined with Pareto optimality this determines exactly the uniform rule $U^{r}$. For later reference, we state this observation as a lemma. ${ }^{8} 9$

\footnotetext{
${ }^{8}$ Schummer and Thomson (1997) note that the uniform reallocation rule assigns the unique Pareto optimal reallocation at which the difference between the smallest and the largest allotment change received by any agent is minimal. Furthermore, they remark that the uniform reallocation rule assigns the unique Pareto optimal reallocation at which the variance of the agents' allotment changes is minimal.

${ }^{9}$ Similar to the uniform allocation rule, the uniform reallocation rule can be seen as a Walrasian solution. Thomson (1995d) states that for exchange economies with a single commodity, the uniform reallocation rule $U^{r}$ is as a special case of Mas-Colell's (1992) "Walrasian solution with slack".
} 
Lemma 4.1 Let $\varphi$ be a Pareto optimal rule. Then $\varphi=U^{r}$ if and only if for all $N \in \mathcal{N}$, all $e=(R, \omega) \in \mathcal{E}^{N}$, and all non-satiated agents $j \in N$,

(a) if $z(e)>0$, then $\triangle \varphi_{j}(e)=\max \left\{\Delta \varphi_{i}(e) \mid i \in N\right\}$ and

(b) if $z(e)<0$, then $\Delta \varphi_{j}(e)=\min \left\{\Delta \varphi_{i}(e) \mid i \in N\right\}$.

Examples demonstrating the computation of the uniform rule $U^{r}$ are given in Chapter 1, Examples 1.5 and 1.6.

It is easy to prove that the uniform reallocation rule $U^{r}$ is an exterision of the uniform allocation rule $U$ in the following sense.

Lemma 4.2 For all $N \in \mathcal{N}$ and all $(R, \Omega) \in \mathcal{R}^{N} \times \mathbb{R}_{+}$,

$$
U(R, \Omega)=U^{r}\left(R,\left(\frac{\Omega}{|N|}, \cdots, \frac{\Omega}{|N|}\right)\right) .
$$

Remark 4.1 In the remainder of this chapter, we are mainly interested in the uniform rule $U^{r}$, its properties, its characterizations, ${ }^{10}$ and the trade-off between the properties in these characterizations. For further results of the reallocation model, for example other reallocation rules or examples of incompatibility of properties, we refer the reader to Barberà, Jackson, and Neme (1997), Moreno (1996), and Thomson (1995d).

Furthermore, it is worth noting that sometimes allocation rule properties applied to an economy $(R, \Omega)$ are equivalent to the corresponding reallocation rule properties applied to the corresponding economy $\left(R,\left(\frac{\Omega}{|N|}, \ldots, \frac{\Omega}{|N|}\right)\right)$. Hence, using this logical relation between allocation and reallocation rule properties, examples of incompatibility of properties for the reallocation model can be easily constructed from the known examples for the allocation model (see for instance Thomson 1995d, Proposition 3).

Remark 4.2 In the remainder of this chapter we present several characterizations of the uniform reallocation rule that can be seen as extensions of existing characterizations of the uniform allocation rule. Some of the proofs we present here are very similar to the proofs as given in the allocation setting (this will be indicated). However, we would like to emphasize that, it is not possible to just "translate" all proofs from the "allocation language" into the "reallocation language". The reallocation model contains some important structural differences to the allocation model.

For instance, in the reallocation model we can divide the set of agents into the agents on the long side of the market, e.g., demanders in case of excess demand, and agents on the short side of the market, e.g., suppliers and non-traders in case of excess demand. So, in each proof of a characterization of the uniform reallocation rule it has to be shown that agents on the short side of the market receive their peaks. This step in the proofs does not have a counterpart in the allocation model.

Another very important difference between the "reallocation proofs" and the "allocation proofs" is that in the reallocation model it is not true that in proofs the

\footnotetext{
${ }^{10}$ Unless mentioned otherwise, the expression "characterization" implies the logical independence of the characterizing properties. For all characterizations appearing in this chapter, however, the discussion of logical independence of the axioms is postponed to Section 4.6 .
} 
argumients for excess demand economies and excess supply economies are symmetric. This asymmetry in the reallocation model is related to the following observation. In the reallocation model negative allotment changes can occur, and since we do not allow for negative allotments for these changes, the lower bound zero may be "active", i.e., an agent's allotment reaches zero and therefore cannot be further diminished. Positive allotment changes are not bounded from above. As a consequence, depending on the properties that are involved, one case of a proof-the part of the proof where the lower bound zero may be active-is generally somewhat more complicated than the other case. Therefore, if the proofs for excess demand and excess supply economies are very similar up to this asymmetry, we will concentrate on the part of the proof where the lower bound zero is possibly active.

\subsection{Strategy-proofness, no-envy, and peak-onliness}

\subsubsection{Strategy-proofness and no-envy}

Similar to Sprumont (1991), we start our axiomatic analysis with the well-known property of strategy-proofness: no agent ever benefits from misrepresenting his preferences (see also Section 2.2, page 22). ${ }^{11}$ Before we give the formal definition, we introduce some notation.

Let $N, M \in \mathcal{N}$ be such that $N \subseteq M$. Let $M \backslash N=\{i \in M \mid i \notin N\}$. For $R \in \mathcal{R}^{M}$ the restriction $\left(R_{i}\right)_{i \in N} \in \mathcal{R}^{N}$ of $R$ to $N$ is denoted by $R_{N}$. Let $R, \bar{R} \in \mathcal{R}^{N}, i \in N$, and $R_{N \backslash\{i\}}=\bar{R}_{N \backslash\{i\}}$. Then, we call $\bar{R}$ an $z$-deviation from $R$.

Strategy-proofness: For all $N \in \mathcal{N}$, all $e=(R, \omega) \in \mathcal{E}^{N}$, all $\bar{e}=\left(\bar{R}_{1} \omega\right) \in \mathcal{E}^{N}$, and all $j \in N$, if $\bar{R}$ is a $j$-deviation from $R$, then $\varphi_{j}(e) R_{j} \varphi_{j}(\bar{e})$.

As already observed by Benassy (1982) in the context of rationing, the uniform rationing mechanism that corresponds to the uniform reallocation rule $U^{r}$ is strategyproof.

Lemma 4.3 The uniform rule $U^{r}$ satisfies strategy-proofness.

Proof. Let $N \in \mathcal{N}, e=(R, \omega) \in \mathcal{E}^{N}, \bar{e}=(\tilde{R}, \omega) \in \mathcal{E}^{N}$, and $j \in N$ be such that $\vec{R}$ is a $j$-deviation from $R$. In order to prove strategy-proofness of $U^{r}$, we have to show that

$$
U_{j}^{r}(e) R_{j} U_{j}^{r}(\ddot{e}) \text {. }
$$

Case 1: $\quad z(e) \leq 0$. Hence, $U_{j}^{r}(e)=\max \left\{p\left(R_{j}\right), \omega_{j}+\lambda\right\}$, where $\lambda$ solves $\sum_{N} U_{i}^{r}(e)=$ $\sum_{N} \omega_{i}$. If $U_{j}^{r}(e)=p\left(R_{i j}\right)$, then (4.1). Otherwise, $U_{j}^{r}(e)=\omega_{j}+\lambda>p\left(R_{j}\right)$. Hence, $j \in S(e)$. We distinguish two subcases.

\footnotetext{
${ }^{11}$ In game theoretical terms a reallocation rule is strategy-proof if in the direct revelation game form it is a weakly dominant strategy for each agent to announce his true preference relation.
} 
Case 1.1: $p\left(\bar{R}_{j}\right)<\omega_{j}+\lambda$. Observe that agent $j$ is still a supplier in the economy $\bar{e}$. Consequently, $S(\bar{e})=S(e)$ and by feasibility and same-sidedness,

$$
\begin{aligned}
s(\bar{e}) & =\sum_{S(\bar{e})}\left(\omega_{i}-p\left(\bar{R}_{i}\right)\right) \\
& =\left(\omega_{j}-p\left(\bar{R}_{j}\right)\right)+\sum_{S(e) \backslash\{j\}}\left(\omega_{i}-p\left(R_{i}\right)\right) \\
& >-\lambda+\sum_{S(e) \backslash[j\}}\left(\omega_{i}-U_{i}^{r}(e)\right) \\
& =\sum_{S(e)}\left(\omega_{i}-U_{i}^{r}(e)\right) \\
& =\sum_{D(e)}\left(p\left(R_{i}\right)-\omega_{i}\right) \\
& =\sum_{D(\bar{e})}\left(p\left(\bar{R}_{i}\right)-\omega_{i}\right)=d(\bar{e}) .
\end{aligned}
$$

Hence, $z(\bar{e})<0$. Therefore, $U_{j}^{r}(\bar{e})=\max \left\{p\left(\bar{R}_{j}\right), \omega_{j}+\bar{\lambda}\right\}$, where $\bar{\lambda}$ solves $\sum_{N} U_{i}^{r}(\bar{e})=$ $\sum_{N} \omega_{i}$. Because $p\left(\bar{R}_{j}\right)<\omega_{j}+\lambda$ and $p\left(\bar{R}_{i}\right)=p\left(R_{i}\right)$ for $i \in N \backslash\{j\}$ it follows that $\bar{\lambda}=\lambda$. Hence, $U_{j}^{r}(\bar{e})=\omega_{j}+\lambda=U_{j}^{r}(e)$. This implies (4.1).

Case 1.2: $p\left(\bar{R}_{j}\right) \geq \omega_{j}+\lambda$. If $z(\bar{e})<0$, then $U_{j}^{r}(\bar{e})=\max \left\{p\left(\bar{R}_{j}\right), \omega_{j}+\ddot{\lambda}\right\} \geq \omega_{j}+\lambda=$ $U_{j}^{r}(e) \geq p\left(R_{j}\right)$. If $z(\bar{e}) \geq 0$, then $U_{j}^{r}(\bar{e})=\min \left\{p\left(\bar{R}_{j}\right), \omega_{j}+\bar{\lambda}\right\} \geq \omega_{j}+\lambda=U_{j}^{r}(\bar{e}) \geq p\left(R_{j}\right)$. This implies (4.1).

Case 2: $\quad z(e)>0$. Similar to Case 1 .

It is easy to show, that the proportional rule $\operatorname{Pr}_{r}{ }^{r}$ is not strategy-proof.

Next, we prove some implications of Pareto optimality and strategy-proofness. First we show that Pareto optimality and strategy-proofness imply own-peak monotonicity: an agent's allotment is monotonic with respect to his peak.

Own-peak monotonicity: For all $N \in \mathcal{N}$, all $e=(R, \omega) \in \mathcal{E}^{N}$, all $\bar{e}=\left(\bar{R}_{x} \omega\right) \in \mathcal{E}^{N}$, and all $j \in N$, if $\bar{R}$ is a $j$-deviation from $R$ and $p\left(R_{j}\right) \leq p\left(\vec{R}_{j}\right)$, then $\varphi_{j}(e) \leq \varphi_{j}(\bar{e})$.

Ching introduced own-peak monotonicity for allocation rules and proved that it is implied by Pareto optimality and strategy-proofness (see Ching 1994, Lemma 1). This result extends to reallocation rules and the structure of our proof is similar to Ching's proof.

Lemma 4.4 Let the rule $\varphi$ satisfy Pareto optimality and strategy-proofness. Then $\varphi$ satisfies own-peak monotonicity.

Proof. Let $\varphi$ be a rule that satisfies Pareto optimality and strategy-proofness. Let $N \in \mathcal{N}, e=(R, \omega) \in \mathcal{E}^{N}, \bar{e}=(\bar{R}, \omega) \in \mathcal{E}^{N}$, and $j \in N$ be such that $\bar{R}$ is a $j$-deviation from $R$ and $p\left(R_{j}\right) \leq p\left(\bar{R}_{j}\right)$. In order to prove own-peak monotonicity of $\varphi$, we have to show that $\varphi_{j}(e) \leq \varphi_{j}(\bar{e})$. Suppose, by contradiction, that

$$
\varphi_{j}(e)>\varphi_{j}(\tilde{e}) \text {. }
$$


Strategy-proofness for the $j$-deviation $\bar{R}$ from $R$ implies $\varphi_{j}(e) R_{j} \varphi_{j}(\bar{e})$. Hence, by (4.2), $p\left(R_{j}\right)>\varphi_{j}(\bar{e})$. Strategy-proofness for the $j$-deviation $R$ from $\bar{R}$ implies $\varphi_{j}(\bar{e}) \bar{R}_{j} \varphi_{j}(e)$. Hence, by $(4.2), p\left(\bar{R}_{j}\right)<\varphi_{j}(e)$.

Thus, by $p\left(R_{j}\right) \leq p\left(\tilde{R}_{j}\right)$, it follows that $\varphi_{j}(\bar{e})<p\left(R_{j}\right) \leq p\left(\bar{R}_{j}\right)<\varphi_{j}(e)$.

Since $p\left(R_{j}\right)<\varphi_{j}(e)$, by same-sidedness it follows that for all $i \in N, p\left(R_{i}\right) \leq \varphi_{i}(e)$. Similarly, since $p\left(\bar{R}_{j}\right)>\varphi_{j}(e)$, by same-sidedness it follows that for all $i \in N, p\left(\bar{R}_{i}\right) \geq$ $\varphi_{i}(\bar{e})$.

Because for all $i \in N \backslash\{j\}, p\left(R_{i}\right)=p\left(\bar{R}_{i}\right)$, it follows that for all $i \in N \backslash\{j\}$, $\varphi_{i}(\bar{e}) \leq \varphi_{i}(e)$. Furthermore, by $(4.2), \varphi_{j}(\bar{e})<\varphi_{j}(e)$. Since $\varphi(e)$ and $\varphi(\bar{e})$ are reallocations of economies with the same endowment vector $\omega$, it follows that $\sum_{N} \omega_{i}=$ $\sum_{N} \varphi_{i}(\bar{e})<\sum_{N} \varphi_{i}(e)=\sum_{N} \omega_{i}$. This is a contradiction.

An inmediate, but important, consequence of own-peak monotonicity of a rule $\varphi$ is individual peak-onliness: if an agent changes his preference relation without changing his peak, his allotment does not change.

Individual peak-onliness: For all $N \in \mathcal{N}$, all $e=(R, \omega) \in \mathcal{E}^{N}$, all $\bar{e}=(\bar{R}, \omega) \in \mathcal{E}^{N}$, and all $j \in N$, if $\tilde{R}$ is a $j$-deviation from $R$ and $p\left(R_{j}\right)=p\left(\bar{R}_{j}\right)$, then $\varphi_{j}(e)=\varphi_{j}(\bar{e})$.

Next, we show that Pareto optimality and strategy-proofness imply uncompromisingness, a bounded invariance property: if an agent's peak differs from the allotment assigned by the rule, then his allotment does not change if he unilaterally deviates from his original preference relation in such a way that his new peak and his original peak are at the same side of the allotment.

Uncompromisingness: For all $N \in \mathcal{N}$, all $e=(R, \omega) \in \mathcal{E}^{N}$, all $\bar{e}=(\vec{R}, \omega) \in \mathcal{E}^{N}$, and all $j \in N$, if $\tilde{R}$ is a $j$-deviation from $R$ and either $\left[p\left(R_{j}\right)>\varphi_{j}(e)\right.$ and $\left.p\left(\bar{R}_{j}\right) \geq \varphi_{j}(e)\right]$ or $\left\{p\left(R_{j}\right)<\varphi_{j}(e)\right.$ and $\left.p\left(\bar{R}_{j}\right) \leq \varphi_{j}(e)\right]$, then $\varphi_{j}(e)=\varphi_{j}(\bar{e})$.

Uncompromisingness is a well-known property in connection with strategy-proofness (see for instance Border and Jordan (1983) in the context of public good economies). Ching considered uncompromisingness for allocation rules and proved that it is implied by Pareto optimality and strategy-proofness (see Ching 1994, Lemma 2). This result extends to reallocation rules and the structure of our proof is similar to Ching's proof.

Lemma 4.5 Let the rule $\varphi$ satisfy Pareto optimality and strategy-proofness. Then $\varphi$ satisfies uncompromisingness.

Proof. Let $\varphi$ be a rule that satisfies Pareto optimality and strategy-proofness. Let $N \in \mathcal{N}, e=(R, \omega) \in \mathcal{E}^{N}, \bar{e}=(\bar{R}, \omega) \in \mathcal{E}^{N}$, and $j \in N$ be such that $\bar{R}$ is a $j$-deviation from $R$.

Case 1: $p\left(R_{j}\right)<\varphi_{j}(e)$ and $p\left(\bar{R}_{j}\right) \leq \varphi_{j}(e)$. By same-sidedness, it follows that for all $i \in N, p\left(R_{i}\right) \leq \varphi_{i}(e)$. In order to prove uncompromisingness of $\varphi$, we have to show that $\varphi_{j}(e)=\varphi_{j}(\bar{e})$. Suppose, by contradiction, that $\varphi_{j}(e) \neq \varphi_{j}(\bar{e})$. 
If $\varphi_{j}(\bar{e})>\varphi_{j}(e)$, then it follows that $p\left(\bar{R}_{j}\right) \leq \varphi_{j}(e)<\varphi_{j}(\bar{e})$. Hence, in contradiction to strategy-proofness, $\varphi_{j}(e) \bar{P}_{j} \varphi_{j}(\bar{e})$.

If $\varphi_{j}(\bar{e})<\varphi_{j}(e)$, then let $e^{\prime}=\left(R^{\prime}, \omega\right) \in \mathcal{E}^{N}$, be such that $R^{\prime}$ is a $j$-deviation from $R$ with $p\left(R_{j}^{\prime}\right)=p\left(R_{j}\right)$ and $\varphi_{j}(\bar{e}) P_{j}^{\prime} \varphi_{j}(e)$. By individual peak-onliness it follows that $\varphi_{j}\left(e^{t}\right)=\varphi_{j}(e)$. Hence, for the $j$-deviation $\bar{R}$ from $R^{\prime}$ it follows that $\varphi_{j}(\bar{e}) P_{j}^{\prime} \varphi_{j}\left(e^{\prime}\right)$. This is a contradiction to strategy-proofness.

Case 2: $\quad p\left(R_{j}\right)>\varphi_{j}(e)$ and $p\left(\bar{R}_{j}\right) \geq \varphi_{j}(e)$. Similar to Case 1 .

The next property we analyze is no-envy (see also Section 2.2, page 22). In contrast with the allocation model, agents are now not only characterized by their preferences, but also by their individual endowments. One could interpret these individual endowments as individual rights. In order to incorporate the individual endowments into our notion of no-envy, we formulate no-envy in terms of trades or allotment changes rather than in terms of net allotments: no agent strictly prefers the allotment change of another agent, or the part of another agent's allotment change that is feasible for him, to his own allotment change.

Let $\alpha \in \mathbb{R}$. Then, $\alpha^{+}:=\max \{0, \alpha\}$.

No-envy: For all $N \in \mathcal{N}$, all $e=(R, \omega) \in \mathcal{E}^{N}$, and all $i, j \in N, \varphi_{j}(e) R_{j}\left(\omega_{j}+\right.$ $\left.\Delta \varphi_{i}(e)\right)^{+}$.

So, agent $j$ only envies agent $i$ if he prefers $i$ 's allotment change, or the part of $i$ 's allotment change that is feasible for him, to his own allotment change.

The well-known property of no-envy can be traced back to Foley (1967) who considered it the context of resource allocation problems. A concept of no-envy in terms of allotment changes-called fair net trade-as introduced above was formulated by Schmeidler and Vind (1972) in the more general context of exchange economies.

Lemma 4.6 The uniform rule $U^{r r}$ satisfies no-envy.

Proof. Let $N \in \mathcal{N}$ and $e=(R, \omega) \in \mathcal{E}^{N}$. In order to prove nowenvy of $U^{r}$, we have to show that for all $i, j \in N$,

$$
U_{j}^{r}(e) R_{j}\left(\omega_{j}+\Delta U_{i}^{r}(e)\right)^{+} .
$$

Case 1: $z(e) \leq 0$. Hence, $U_{j}^{r}(e)=\max \left\{p\left(R_{j}\right), w_{j}+\lambda\right\}$, where $\lambda$ solves $\sum_{N} U_{i}^{r}(e)=$ $\sum_{N} w_{i}$. If $U_{j}^{r}(e)=p\left(R_{j}\right)$, then (4.3). Otherwise, $U_{j}^{r}(e)=w_{j}+\lambda>p\left(R_{j}\right)$. Hence, agent $j$ is non-satiated at $U^{r}(e)$. Then, by Lemma 4 , for all $i \in N, \Delta U_{j}^{r}(e) \leq \Delta U_{i}^{r}(e)$. Since $U_{j}^{r}(e)=\omega_{j}+\Delta U_{j}^{r}(e) \geq 0$, it follows that for all $i \in N, \omega_{j}+\Delta U_{i}^{r}(e) \geq 0$. Hence, for all $i \in N, p\left(R_{j}\right)<U_{j}^{r}(e)=\omega_{j}+\Delta U_{j}^{r}(e) \leq \omega_{j}+\Delta U_{i}^{r}(e)=\left(\omega_{j}+\Delta U_{i}^{r}(e)\right)^{+}$. This implies (4.3).

Case 2: $z(e)>0$. Similar to Case 1 .

Next, we introduce the slightly weaker notion of weak no-envy, 
Weak no-envy: For all $N \in \mathcal{N}$, all $e=(R, \omega) \in \mathcal{E}^{N}$, and all $i, j \in N$ with $\omega_{j}+\Delta \varphi_{i}(e) \geq 0, \varphi_{j}(e) R_{j}\left(\omega_{j}+\Delta \varphi_{i}(e)\right)$.

So, agent $j$ only envies agent $i$ if he prefers $i$ 's allotment change, added to his endowment, to his own allotment-provided the former is feasible.

It is easy to show that the proportional rule $P r^{\pi}$ satisfies neither no-envy nor weak no-envy.

No-envy, for allocation problems with single-peaked preferences, is one of the conditions that led Sprumont (1991) to one of his characterizations of the uniform allocation rule: the uniform rule $U$ is the only rule that satisfes Pareto optimality, strategyproofness, and nomenvy (see Theorem 2.2). Ching (1992, Theorem 1) gives a simple and elegant proof of this result. Next, we show that this result extends to reallocation rules.

Theorem 4.1 The uniform rule $U^{T}$ is the only rule that satisfies Pareto optimality, strategy-proofness, and no-envy.

One possibility to prove Theorem 4.1 is to adjust Ching's proof for the allocation model to our reallacation model. The interested reader can find this version of the proof in Appendix B. Here, we present a proof of Theorem 4.1 that is structured similarly to the proof of Ching's characterization of the uniform allocation rule by Pareto optimality, equal treatment of equals, and strategy-proofness (see Theorem 2.4). The advantage of this somewhat longer proof is that by only small adjustments of the proof we can easily derive some related results (see Corollaries $4.1,4.2$, and 4.5 ).

Proof. The uniform rule $U^{r}$ is same-sided and therefore Pareto optimal. As shown in Lemmas 4.3 and $4.6, U^{r}$ satisfies strategy-proofness and no-envy.

To prove the remaining part of the theorem let $\varphi$ be a rule that satisfies the properties listed in the theorem. By Lemmas 4.4 and 4.5, both $\varphi$ and $U^{r}$ satisfy own-peak monotonicity and uncompromisingness.

Let $N \in \mathcal{N}, e=(R, \omega) \in \mathcal{E}^{N}$, and suppose, by contradiction, that $\varphi(e) \neq U^{r}(e)$. Since $\varphi$ is Pareto optimal it follows that either $z(e)>0$ or $z(e)<0$.

Case 1: $z(e)>0$. We call a demander $j$ a maximal demander if $j \in \arg \max \left\{d_{i}(e)\right.$ $i \in D(e)\}$. We denote the set of maximal demanders of an economy $e \in \mathcal{E}^{N}$ by $D_{\max }(e)$. By $d_{\max }:=\max \left\{d_{i}(e) \mid i \in D(e)\right\}$ we denote the maximal demand.

Case 1.1: All agents are maximal demanders, i.e., $D_{\max }(e)=N$. Hence, $U^{r}(e)=\omega$ and for all $i \in N, d_{i}(e)=d_{\max }$.

Since $\varphi(e) \neq U^{r}(e)$, by Lemma 4.1 , there exist agents $j, k \in N$ such that $\varphi_{j}(e)<$ $p\left(R_{j}\right)$ and $\Delta \varphi_{j}(e)<\Delta \varphi_{k}(e)$. Same-sidedness implies that $\Delta \varphi_{k}(e) \leq d_{\max }$. Hence, $\varphi_{j}(e)=\omega_{j}+\Delta \varphi_{j}(e)<\omega_{j}+\Delta \varphi_{k}(e)=\left(\omega_{j}+\Delta \varphi_{k}\left(e_{j}\right)^{+} \leq p\left(R_{j}\right)\right.$. Thus, in contradiction to no-envy, $\left(\omega_{j}+\Delta \varphi_{k}(e)\right) P_{j} \varphi_{j}(e)$.

Case 1.2: The set of maximal demanders does not equal the set of agents, i.e., $D_{\max }(e) \subseteq N$. 
Since $\varphi(e) \neq U^{r}(e)$, by Lemma 4.I, there exist agents $j, k \in N$ such that $\varphi_{j}(e)<$ $p\left(R_{j}\right)$ and $\Delta \varphi_{j}(e)<\Delta \varphi_{k}(e)$. Furthermore, by the same argument as in Case 1.1, $j \notin D_{\max }(e)$. Let $\vec{e}=(\bar{R}, \omega) \in \mathcal{E}^{N}$ such that $\bar{R}$ is a $j$-deviation from $R$ with $p\left(\vec{R}_{j}\right)=$ $\omega_{j}+d_{\max }$. We consider the following two subcases.

Case 1.2.1: $\varphi_{j}(e)<U_{j}^{r}(e) \leq p\left(R_{j}\right)$. Since $p\left(R_{j}\right)<p\left(\bar{R}_{j}\right)$, by uncompromisingness of $\varphi$ it follows that $\varphi_{j}(\bar{e})=\varphi_{j}(e)$. Own-peak monotonicity of $U^{r}$ yields $U_{j}^{r}(e) \leq U_{j}^{r}(\bar{e})$. Hence, $\varphi_{j}(\bar{e})<U_{j}^{r}(\bar{e})$.

Case 1.2.2: $\quad U_{j}^{r}(e)<\varphi_{j}(e) \leq p\left(R_{j}\right)$. Similar to Case 1.2.1 (exchange the roles of $U^{r}$ and $\varphi$ ) it follows that $\varphi_{j}(\bar{e})>U_{j}^{r}(\bar{e})$.

Thus, for the economy $\bar{e}$ it follows that $\varphi_{j}(\bar{e}) \neq U_{j}^{r}(\bar{e})$. Note that the economy $\bar{e}$ has one maximal demander more than the economy e, i.e., $\left|D_{\max }(\bar{e})\right|=\left|D_{\max }(e)\right|+1$. By Case 1.1, $D_{\max }(\bar{e}) \subseteq N$.

Similarly, proceeding from the economy $\bar{e}$, we consider a unilateral change of an agent's preference relation such that the set of maximal demanders increases by one and the uniform reallocation for this economy is unequal to the reallocation assigned by $\varphi$, etc. Since the set of agents is finite, finally we end up in an economy $e^{\prime}$ with only maximal demanders and $\varphi\left(e^{\prime}\right) \neq U^{r}\left(e^{r}\right)$. By Case 1.1, this is a contradiction.

Case 2: $z(e)<0$. We call an agent $j \in S(e)$ a maximal supplier if either $j \in$ $\arg \max \left\{s_{i}(e) \mid i \in S(e)\right\}$ or $p\left(R_{j}\right)=0$ (here, in contrast to Case 1, the lower bound zero may be active). We denote the set of maximal suppliers of an economy $e \in \mathcal{E}^{N}$ by $S_{\max }(e)$. By $s_{\max }=\max \left\{s_{i}(e) \| i \in S(e)\right\}$ we denote the maximal supply.

Case 2.1: All agents are maximal suppliers, i.e., $S_{\max }(e)=N$. Hence, $U^{r}(e)=\omega$.

Since $\varphi(e) \neq U^{r}(e)$, by Lemma 4.1 , there exist agents $j, k \in N$ such that $p\left(R_{j}\right)<$ $\varphi_{j}(e)$ and $\Delta \varphi_{j}(e)>\Delta \varphi_{k}(e)$. Same-sidedness implies that $\Delta \varphi_{k}(e) \geq s_{\max }$. If $j \in$ $\arg \max \left\{s_{i}(e) \mid i \in S(e)\right\}$, then $\varphi_{j}(e)=\omega_{j}+\Delta \varphi_{j}(e)>\omega_{j}+\Delta \varphi_{k}(e)=\left(\omega_{j}+\Delta \varphi_{k}(e)\right)^{+} \geq$ $p\left(R_{j}\right)$. Hence, in contradiction to no-envy, $\left(\omega_{j}+\Delta \varphi_{k}(e)\right)^{+} P_{j} \varphi_{j}(e)$. If $p\left(R_{j}\right)=0$, then $\varphi_{j}(e)=\omega_{j}+\Delta \varphi_{j}(e)>\left(\omega_{j}+\Delta \varphi_{k}(e)\right)^{+} \geq 0=p\left(R_{j}\right)$. Hence, in contradiction to no-erivy, $\left(\omega_{j}+\Delta \varphi_{k}(e)\right)^{+} P_{j} \varphi_{j}(e)$.

Case 2.2: The set of maximal suppliers does not equal the set of agents, i.e., $S_{\max }(e) \subseteq$ $N$.

Since $\varphi(e) \neq U^{r}(e)$, by Lemma 4.1, there exist agents $j, k \in N$ such that $p\left(R_{j}\right)<$ $\varphi_{j}(e)$ and $\Delta \varphi_{j}(e)>\Delta \varphi_{k}(e)$. Furthermore, by the same argument as in Case 2.1, $j \notin S_{\max }(e)$. Let $\bar{e}=(\bar{R}, \omega) \in \mathcal{E}^{N}$ such that $\widetilde{R}$ is a $j$-deviation from $R$ with $p\left(\bar{R}_{j}\right)=$ $\max \left\{0, \omega_{j}-s_{\max }\right\}$. We consider the following two subcases.

Case 2.2.1: $\quad \varphi_{j}(e)>U_{j}^{\tau}(e) \geq p\left(R_{j}\right)$. Since $p\left(R_{j}\right)>p\left(\bar{R}_{j}\right)$, by uncompromisingness of $\varphi$ it follows that $\varphi_{j}(\bar{e})=\varphi_{j}(e)$. Own-peak monotonicity of $U^{r}$ yields $U_{j}^{r}(e) \geq U_{j}^{r}(\bar{e})$. Hence, $\varphi_{j}(\bar{e})>U_{j}^{r}(\bar{e})$.

Case 2.2.2: $\quad U_{j}^{r}(e)>\varphi_{j}(e) \geq p\left(R_{j}\right)$. Similar to Case 2.1 (exchange the roles of $U^{r}$ and $\varphi$ ) it follows that $\varphi_{j}(\bar{e})<U_{j}^{r}(\bar{e})$.

Thus, for the economy $\bar{e}$ it follows that $\varphi_{j}(\bar{e}) \neq U_{j}^{r}(\bar{e})$. Note that the economy $\bar{e}$ has one maximal supplier more than the economy e, i.e., $\left|S_{\max }(\bar{e})\right|=\left|S_{\max }(e)\right|+1$. By Case $2.1, S_{\max }(\bar{e}) \subsetneq N$. 
Similarly as in the proof of Case 1 we now construct, step by step, an economy $e^{\prime}$ with only maximal suppliers and $\varphi\left(e^{\prime}\right) \neq U^{r}\left(e^{\prime}\right)$. By Case 2.1 , this is a contradiction.

It is easy to check that, since the lower bound zero is not active in Case 1 of the previous proof, we can replace no-envy by weak no-nvy. This implies the following corollary.

Corollary 4.1. Let $\varphi$ be a rule that satisfies Pareto optimality, strategy-proofness, and weak no-envy. Then, for all $N \in \mathcal{N}$ and all $e=(R, \omega) \in \mathcal{E}^{N}$ such that $z(e) \geq 0$, $\varphi(e)=U^{r}(e)$.

Next, we introduce an equal treatment of equals condition. The notion of equal treatment of equals we introduce here for the reallocation model is somewhat different from the equal treatment condition as introduced in Chapter 2 for the allocation model (see Section 2.2, page 23). There, we considered two agents to be equal if they have the same preference relations. However, in the reallocation model, we introduce a notion of "equal agents" in terms of demands rather than in terms of preferences. ${ }^{12}$ Equal treatment of equals requires that if two agents have the same demand, then each of them is indifferent between his allotment change and the feasible part of the other agent's allotment change.

Equall treatment of equals: For all $N \in \mathcal{N}$, all $e=(R, \Omega) \in \mathcal{E}^{N}$, and all $i, j \in N$, if $d_{i}(e)=d_{j}(e)$, then $\left(\omega_{i}+\Delta \varphi_{i}(e)\right) I_{i}\left(\omega_{i}+\Delta \varphi_{j}(e)\right)^{+}$.

If $\varphi$ is in addition Pareto optimal, then equal treatment of equals implies the stronger equality condition $\omega_{i}+\Delta \varphi_{i}(e)=\left(\omega_{i}+\Delta \varphi_{j}(e)\right)^{+}$. It is easy to check that equal treatment of equals is a weakening of no-envy and that equal treatment of equals and weak no-envy are logically independent.

The uniform rule $U^{r}$ and the proportional rule $P r^{r}$ satisfy equal treatment of equals.

In the proof of Theorem 4.1, Case 1 , we show that if the set of agents consists only of maximal demanders, then the reallocation recommended by the rule in question equals the uniform reallocation. Since in such an economy, all agents have the same demand, we can replace no-envy by equal treatment of equals without changing the result. Hence, the following holds.

Corollary 4.2 Let $\varphi$ be a rule that satisfies Pareto optinality, strategy-proofness, and equal treatment of equals. Then, for all $N \in \mathcal{N}$ and ab $e=(R, \omega) \in \mathcal{E}^{N}$ such that $z(e) \geq 0, \varphi(e)=U^{r}(e)$.

The following example shows that it is not passible to weaken no-envy in Theorem 4.1. to either weak no-envy or equal treatment of equals.

\footnotetext{
${ }^{12}$ In Chapter 5 , for variations of the reallocation model where preferences are defined over the whole real line, we introduce a stronger equal treatment of equals condition in terms of individual enclowments together with preferences rather than in terms of the agents" demands.
} 
Example 4.1 The following rule $\varphi^{1}$ satisfies Pareto optimality, strategy-proofness, weak no-envy, and equal treatment of equals. If $T=\{1,2\}, e=(R, \omega) \in \mathcal{E}^{T}$, and $z(e)<0$, then

$$
\varphi^{1}(e)= \begin{cases}\left(\omega_{2}-p\left(R_{2}\right), p\left(R_{2}\right)\right) & \text { if } \omega_{1}=0 \\ \left(p\left(R_{1}\right), \omega_{1}-p\left(R_{1}\right)\right. & \text { if } \omega_{2}=0 \\ U^{r}(e) & \text { otherwise. }\end{cases}
$$

For all remaining $N \in \mathcal{N}$ and $e=(R, \omega) \in \mathcal{E}^{N}, \varphi^{1}(e)=U^{r}(e)$.

\subsubsection{Endowment strategy-proofness}

Next, we introduce a notion of strategy-proofness that has no counterpart in the allocation model. If individual endowments are private information, it might happen that an agent manipulates the outcome to his advantage by only reporting, or exhibiting, a smaller part of his endowment. An example for a rule that is vulnerable to this kind of strategic behavior is the proportional rule $\operatorname{Pr}^{r}$. Endowment strategy-proofness states that no agent ever benefits from withholding some part of his individual endowment. Before we give the formal definition, we introduce some notation.

Let $N, M \in \mathcal{N}$ be such that $N \subseteq M$. For $\omega \in \mathbb{R}_{+}^{M}$ the restriction $\left(\omega_{i}\right)_{i \in N} \in \mathbb{R}_{+}^{N}$ of $\omega$ to $N$ is denoted by $\omega_{N}$. Let $\omega, \bar{\omega} \in \mathbb{R}_{+}^{N}$ such that for $i \in N, \omega_{N \backslash\{i\}}=\bar{\omega}_{N \backslash\{i\}}$. Then, we call $\bar{\omega}$ an $i$-deviation from $\omega$. If in addition $\omega_{i}>\bar{\omega}_{i}$, then, we call $\bar{\omega}$ an i-deviation from $\omega$ by withholding.

Endowment strategy-proofness: For all $N \in \mathcal{N}$, all $e=(R, \omega) \in \mathcal{E}^{N}$, all $\vec{e}=$ $(R, \bar{\omega}) \in \mathcal{E}^{N}$, and all $j \in N$, if $\bar{\omega}$ is a $j$-deviation from $\omega$ by withholding, then $\varphi_{j}(e) R_{j}\left(\omega_{j}+\Delta \varphi_{j}(\bar{e})\right)$.

So, if agent $j$ acts strategically and pretends to have the individual endowment $\bar{\omega}_{i}$ instead of $\omega_{i}$, then the allotment assigned by the rule equals $\varphi_{j}(\bar{e})$. If endowments are private information, $\varphi$ can better be interpreted as a rule that assigns allotment changes. So, $j$ 's actual allotment in that situation is $\left(\omega_{i}+\Delta \varphi_{j}(\bar{e})\right)$.

If agents were also allowed to offer more than their actual possession, then feasibility could cause some agents to obtain a negative allotment. Because our model does not allow such negative allotments and supplies are considered to be real amounts handed out, demands can be faked only by withholding endowment.

The property of endowment strategy-proofness can be traced back to Postlewaite (1979), who investigated it for pure exchange economies.$^{13}$ In this context, endowment strategy-proof mechanisms do not exist (see Postlewaite 1979, Theorem 1). For our model with single-peaked preferences endowment strategy-proof rules do exist, e.g., the uniform rule $U^{r}$.

Lemma 4.7 The uniform rule $U^{r}$ satisfies endowment strategy-proofness.

\footnotetext{
${ }^{13}$ In Postlewaite (1979) the manipulation by witholding some part of an individual endowment is called W-manipulability.
} 
Proof. Let $N \in \mathcal{N}, e=(R, \omega) \in \mathcal{E}^{N}, \bar{e}=(\bar{R}, \omega) \in \mathcal{E}^{N}$, and $j \in N$ be such that $\bar{\omega}$ is a $j$-deviation from $\omega$ by withholding. In order to prove endowment strategy-proofness of $U^{T}$, we have to show that

$$
U_{j}^{r}(e) R_{j}\left(\omega_{j}+\Delta U_{j}^{r}(\bar{e})\right)
$$

Case 1: $z(e) \leq 0$. Hence, $U_{j}^{r}(e)=\max \left\{p\left(R_{j}\right), \omega_{j}+\lambda\right\}$, where $\lambda$ solves $\sum_{N} U_{i}^{r}(e)=$ EN $\omega_{i}$. Then, by samesidedness, for all $i \in N, U_{i}^{r}(e) \geq p\left(R_{i}\right)$. If $U_{j}^{r}(e)=p\left(R_{j}\right)$, then (4.4). Otherwise, $j \in S(e)$ and $U_{j}^{r}(e)=\omega_{j}+\lambda>p\left(R_{j}\right)$. We distinguish two cases.

Case 1.1: $z(\bar{e}) \leq 0$. Then, by the definition of $U^{r}, \Delta U_{j}^{r}(e) \leq \Delta U_{j}^{r}(\bar{e})$. This implies (4.4).

Case 1.2: $z(\bar{e})>0$. Hence, by same-sidedness, for all $i \in N, U_{i}^{r}(\bar{e}) \leq p\left(R_{i}\right)$. By feasibility, it follows that $\sum_{N} U_{i}^{r}(\ddot{e})=\sum_{N} \bar{\omega}_{i}=\sum_{N} \omega_{i}+\bar{\omega}_{j}-\omega_{j}=\sum_{N} U_{i}^{r}(e)+\bar{\omega}_{j}-\omega_{j}$. This implies that $U_{j}^{r}(\bar{e})-\bar{\omega}_{j}=U_{j}(e)-\omega_{j}+\sum_{N\{j\}}\left(U_{i}^{r}(e)-U_{i}^{r}(\bar{e})\right)$. Since for all $i \epsilon$ $N \backslash\{j\}, U_{i}^{r}(e)-U_{i}^{r}(\bar{e}) \geq 0$, we have $U_{j}^{r}(\bar{e})-\bar{\omega}_{j} \geq U_{j}^{r}(e)-\omega_{j}$. Hence, $\Delta U_{j}^{r}(e) \leq \Delta U_{j}^{r}(\bar{e})$. This implies (4.4).

Case 2: $\quad z(e)>0$. Similar to Case 1 .

It is easy to show, that the proportional rule $P r^{r}$ is not endowment strategy-proof.

The following lemma describes some implications of endowment strategy-proofness for the case that a deviation from $\omega$ by withholding is a "one-sided change" of the economy: let $N, \bar{N} \in \mathcal{N}, e=(R, \omega) \in \mathcal{E}^{N}$, and $\bar{e}=(\bar{R}, \bar{\omega}) \in \mathcal{E}^{\bar{N}}$. If $z(e) \cdot z(\bar{e}) \geq 0$, then $\bar{e}$ is a one-sided change of $e$.

Lemma 4.8 Let the rule $\varphi$ satisfy Pareto optimality and endowment strategy-proofness. Let $N \in \mathcal{N}, e=(R, \omega) \in \mathcal{E}^{N}, \bar{e}=(R, \vec{\omega}) \in \mathcal{E}^{N}$, and $j \in N$ be such that $\bar{e}$ is a one-sided change of $e, \omega$ is a $j$-deviation from $\omega$ by witholding, and $j$ is non-satiated at $e, i e$. $\varphi_{j}(e) \neq p\left(R_{j}\right)$.

(a) If $z(\bar{e}) \leq 0$, then $\Delta \varphi_{j}(\bar{e}) \geq \Delta \varphi_{j}(e)$.

(b) If $z(e) \geq 0$, then $\Delta \varphi_{j}(e) \leq \Delta \varphi_{j}(e)$.

Proof. Let $\varphi$ be a rule that satisfies Pareto optimality and endowment strategyproofness. Let $N \in \mathcal{N}, e=(R, \omega) \in \mathcal{E}^{N}, \bar{e}=(R, \bar{\omega}) \in \mathcal{E}^{N}$, and $j \in N$ be as described in the lemma.

(a) Suppose $z(\bar{e}) \leq 0$. Then, $z(e)<0$. By same-sidedness, $\varphi_{j}(e)>p\left(R_{j}\right)$ and $\varphi_{j}(\tilde{e}) \geq p\left(R_{j}\right)$. By endowment strategy-proofness, $\varphi_{j}(e) R_{j}\left(\omega_{j}+\Delta \varphi_{j}(\bar{e})\right)$. Since $\omega_{j}>$ $\bar{\omega}_{j}$, it follows that $\omega_{j}+\Delta \varphi_{j}(\bar{e}) \geq \varphi_{j}(e)>p\left(R_{j}\right)$. Hence, $\Delta \varphi_{j}(\bar{e}) \geq \Delta \varphi_{j}(e)$.

(b) Suppose $z(e) \geq 0$. Then, $z(\bar{e})>0$. By same-sidedness, $\varphi_{j}(e)<p\left(R_{j}\right)$ and $\varphi_{j}(\tilde{e}) \leq p\left(R_{j}\right)$. By endowment strategy-proofness, $\varphi_{j}(e) R_{j}\left(\omega_{j}+\Delta \varphi_{j}(\bar{e})\right)$. Since $\omega_{j}>$ $\bar{\omega}_{j}$, it follows that either (i) $\omega_{j}+\left(\varphi_{j}(\bar{e})-\bar{\omega}_{j}\right) \leq \varphi_{j}(e)$ or $\left(\right.$ ii) $\omega_{j}+\left(\varphi_{j}(\bar{e})-\bar{\omega}_{j}\right) \geq r_{j}\left(\varphi_{j}(e)\right)$. Suppose $\omega_{j}-\dot{\omega}_{j}<r_{j}\left(\varphi_{j}(e)\right)-p\left(R_{j}\right)$. Then, $\omega_{j}-\bar{\omega}_{j}<r_{j}\left(\varphi_{j}(e)\right)-\varphi_{j}(\bar{e})$. Hence, (ii) cannot be the case and (i) implies $\Delta \varphi_{j}(\bar{e}) \leq \Delta \varphi_{j}(e)$. Finally, if $\omega_{j}-\bar{\omega}_{j} \geq r_{j}\left(\varphi_{j}(e)\right)-p\left(R_{j}\right)$, we shift $\omega_{j}$ stepwise (with the size of the steps small enough) to $\bar{\omega}_{j}$ and apply the same argument as above in each step. 
We have the following consequence of the previous lemma.

Corollary 4.3 Let the rule $\varphi$ satisfy Pareto optimality and endowment strategy-proofness. Let $N \in \mathcal{N}$ and $e=(R, \omega) \in \mathcal{E}^{N}$ be such that $z(e)>0$. Then,

(a) for all $i \in D(e), \omega_{i} \leq \varphi_{i}(e) \leq p\left(R_{i}\right)$ and

(b) for all $i \notin D(e), \varphi_{i}(e)=p\left(R_{i}\right)$.

Proof. Let $\varphi$ be a rule that satisfies Pareto optimality and endowment strategyproofness. Let $N \in \mathcal{N}$ and $e=(R, \omega) \in \mathcal{E}^{N}$ be such that $z(e)>0$. Then, by same-sidedness, for all $i \in N, \varphi_{i}(e) \leq p\left(R_{i}\right)$. Let $j \in N$ and define $\omega(j) \in \mathbb{R}_{+}^{N}$ by $\omega(j)_{j}=0$ and $\omega(j)_{i}=\omega_{i}$ for all $i \in N \backslash\{j\}$. Let $e(j)=(R, \omega(j)) \in \mathcal{E}^{N}$. Let $i \in N$ and suppose that $\varphi_{i}(e)<p\left(R_{i}\right)$. Then, by Lemma $4.8, \Delta \varphi_{i}(e(i)) \leq \Delta \varphi_{i}(e)$. Because $\varphi_{i}(e(i)) \geq 0$, it follows that $\Delta \varphi_{i}(e(i)) \geq 0$. Hence, $\Delta \varphi_{i}(e) \geq 0$ and $\omega_{i} \leq \varphi_{i}(e)<p\left(R_{i}\right)$. Now, (a) and (b) follow easily.

A similar result cannot be obtained for the excess supply case, even if there is an upper bound for the endowments (for instance, if there are finite resources). This is due to the asymmetry in the definition of endowment strategy-proofness caused by the requirement that an agent can only deviate by withholding part of his endowment. Therefore, endowment strategy-proofness as defined here, has not such a great impact for a rule as one would expect: We will use the next consequence of the previous results in the next section.

Corollary 4.4 Let $\varphi$ be a rule that satisfies Pareto optimality, weak no-envy, and endowment strategy-proofness. Let $N \in \mathcal{N}$ and $e=(R, \omega) \in \mathcal{E}^{N}$ be such that $z(e) \geq 0$ and for all $i, j \in N, p\left(R_{i}\right)=p\left(R_{j}\right)$. Then, $\varphi(e)=U^{r}(e)$.

Proof. Let $\varphi$ be a rule that satisfies Pareto optimality and endowment strategyproofness. Let $N \in \mathcal{N}$ and $e=(R, \omega) \in \mathcal{E}^{N}$ be such that $z(e) \geq 0$ and for all $i, j \in N$, $p\left(R_{i}\right)=p\left(R_{j}\right)$. Suppose, by contradiction, that $\varphi(e) \neq U^{r}(e)$. Since $\varphi$ is Pareto optimal it follows that $z(e)>0$. Furthermore, there exist an agent $j \in N$ such that $\Delta \varphi_{j}(e)<$ $\Delta U_{j}^{+}(e)$. By Corollary 4.3 , it follows that $j \in D(e)$ and $\Delta \varphi_{j}(e) \geq 0$. Similar to the proof of Corollary 4.3 , define $\omega(j) \in \mathbb{R}_{+}^{N}$ by $\omega(j)_{j}=0$ and $\omega(j)_{i}=\omega_{i}$ for all $i \in N \backslash\{j\}$. Let $e(j)=(R, \omega(j)) \in \mathcal{E}^{N}$. Then, by Lemma $4.8, \Delta \varphi_{j}(e) \geq \Delta \varphi_{j}(e(j)) \geq 0$. Because $\Delta U_{j}^{r}(e(j)) \geq \Delta U_{j}^{r}(e)$, it follows that $\Delta U_{j}^{r}(e(j))>\Delta \varphi_{j}(e(j))$. Thus, there exists an agent $k \in N$ such that $\Delta U_{k}^{r}(e(j))<\Delta \varphi_{k}(e(j))$. Since $z(e(j))>0$, by Corollary 4.3 , $k \in D(e(j)), \omega_{k} \leq \varphi_{k}(e(j)) \leq p\left(R_{k}\right)$, and $\Delta \varphi_{k}(e(j))>\Delta U_{k}^{r}(e(j)) \geq \Delta U_{j}^{r}(e(j))>$ $\Delta \varphi_{j}(e(j))$. Hence, $\omega(j)_{j}+\Delta \varphi_{j}(e(j))<\omega(j)_{j}+\Delta \varphi_{k}(e(j)) \leq \varphi_{k}(e) \leq p\left(R_{k}\right)=p\left(R_{j}\right)$. This contradicts weak no-envy.

The following example shows that even if we strengthen weak no-envy to no-envy, the result of Corollary 4.4 does not hold for economies with excess supply.

Example 4.2 The following rule $\varphi^{2}$ satisfies Pareto optimality, no-envy, and endowment strategy-proofness. Let $T=\{1,2\}$ and $e=(R, \omega) \in \mathcal{E}^{T}$. 
If $z(e)<0$ and $\omega_{1}=0$, then

$$
\begin{aligned}
& \varphi_{1}^{2}(e)=\min \left\{r_{1}(0), \omega_{2}-p\left(R_{2}\right)\right\} \text { and } \\
& \varphi_{2}^{2}(e)=\max \left\{p\left(R_{2}\right), p\left(R_{2}\right)-r_{1}(0)\right\} .
\end{aligned}
$$

If $z(e)<0$ and $\omega_{2}=0$, then

$$
\begin{aligned}
& \varphi_{1}^{2}(e)=\max \left\{p\left(R_{1}\right), p\left(R_{1}\right)-r_{2}(0)\right\} \text { and } \\
& \varphi_{2}^{2}(e)=\min \left\{r_{2}(0), \omega_{1}-p\left(R_{1}\right)\right\} .
\end{aligned}
$$

For all remaining $N \in \mathcal{N}$ and $e=(R, \omega) \in \mathcal{E}^{N}, \varphi^{2}(e)=U^{r}(e)$.

\subsubsection{Demand-onliness, reversibility, and peak-onliness}

The next property for rules we introduce in this section is demand-onliness: the agents' allotment changes only depend on the agents' demands rather than on the whole economy.

Demand-onliness: For all $N \in \mathcal{N}$, all $e=(R, \omega) \in \mathcal{E}^{N}$, and all $\bar{e}=\left(\bar{R}_{,} \bar{\omega}\right) \in \mathcal{E}^{N}$, if for all $i \in N, d_{i}(e)=d_{i}(\bar{e})$, then for all $i \in N, \Delta \varphi_{i}(e)=\Delta \varphi_{i}(\bar{e})$.

The uniform rule $U^{r}$ and the proportional rule $\mathrm{Pr}^{r}$ satisfy demand-onliness.

The next corollary states that in Theorem 4.1 we can replace no-envy by equal treatment of equals and demand-onliness.

Corollary 4.5 The uniform rule $U^{r}$ is the only rule that satisfies Pareto optimality, strategy-proofness, equal treatment of equals, and demand-onliness.

We can interpret Corollary 4.5 as an extension of Ching's characterization of the uniform allocation rule by Pareto optimality, equal treatment of equals, and strategyproofness (see Theorem 2.4) to the reallocation model. In addition to the corresponding reallocation properties, we impose demand-onliness. By demand-onliness it is sufficient to only consider economies where all agents have identical individual endowments. The remaining part of the proof is then similar to Ching's proof for the allocation model.

Proof. The uniform rule $U^{r}$ satisfies Pareto optimality, strategy-proofness, equal treatment of equals, and demand-onliness.

To prove the remaining part of the corollary let $\varphi$ be a rule that satisfies the properties listed in the corollary. By Pareto optimality and Corollary 4.2, it follows that for all $N \in \mathcal{N}$ and $e=(R, \omega) \in \mathcal{E}^{N}$ such that $z(e) \geq 0, \varphi(e)=U^{r}(e)$. It remains to prove that for all $N \in \mathcal{N}$ and $e=(R, \omega) \in \mathcal{E}^{N}$ such that $z(e)<0, \varphi(e)=U^{r}(e)$. By demandonliness it is sufficient to consider $N \in \mathcal{N}$ and $e=(R, \omega) \in \mathcal{E}^{N}$ such that for all $i, j \in N$, 
$\omega_{i}=\omega_{j}$. Then, for all agents $j, k \in N$ it follows that $\left(\omega_{j}+\Delta \varphi_{k}(e)\right)^{+}=\left(\omega_{j}+\Delta \varphi_{k}(e)\right)$. Now, the remainder of the proof is similar to the proof of Theorem 4.1 by applying equal treatment of equals instead of no-envy.

The rule $\varphi^{1}$ as defined in Example 4.1 satisfies Pareto optimality, strategy-proofness, and equal treatment of equals, but not demand-onliness.

Similar to Theorem 2.4, Sprumont's characterization of the uniform allocation rule by Pareto optimality, strategy-proofness, and anonymity (Theorem 2.1) implies a characterization of the uniform reallocation rule if in addition demand-onliness is imposed. Anonymity in the reallocation model requires that the agent's allotment changes do not depend on their names (see also Section 2.2, page 22).

Let $N \in \mathcal{N}$. A permutation $\pi$ on $N$ is a bijective function $\pi: N \rightarrow N$. By $\Pi^{N}$ we denote the set of all permutations on $N$. Given $\pi \in \Pi^{N}$ and $e=(R, \Omega) \in \mathcal{R}^{N}$, let $R_{\pi}=\left(R_{\pi(i)}\right)_{i \in N}, \omega_{\pi}=\left(\omega_{\pi(i)}\right)_{i \in N}$, and $e_{\pi}=\left(R_{\pi}, \omega_{\pi}\right)$.

Anonymity: For all $N \in \mathcal{N}$, all $e=(R, \omega) \in \mathcal{E}^{N}$, all $\pi \in \Pi^{N}$, and all $i \in N$, $\varphi_{i}\left(e_{\pi}\right)=\varphi_{\pi(i)}(e)$.

Lemma 4.9 Let $\varphi$ satisfy Pareto optimality; anonymity, and demand-onliness. Then $\varphi$ satisfies equal treatment of equals.

Proof. By demand-onliness it is sufficient to consider $N \in \mathcal{N}$ and $e=(R, \omega) \in \mathcal{E}^{N}$ such that for all $i, j \in N, \omega_{i}=\omega_{j}$. Let $i, j \in N$ be such that $d_{i}(e)=d_{j}(e)$. Thus, $p\left(R_{i}\right)=p\left(R_{j}\right)$. By demand-onliness, suppose, without loss of generality, that $R_{i}=R_{j}$. Let $\pi \in \Pi^{N}$ be such that $\pi(i)=j, \pi(j)=i$, and for all $k \in N \backslash\{i, j\}$, $\pi(k)=k$. Hence, $e=e_{\pi}$ and by anonymity, for all $k \in N, \varphi_{k}\left(e_{\pi}\right)=\varphi_{\pi(k)}(e)$. Thus, $\varphi_{i}(e)=\varphi_{i}\left(e_{\pi}\right)=\varphi_{j}(e)$. Since $\omega_{i}=\omega_{j}$, this proves equal treatment of equals.

The next corollary states that in Theorem 4.1 we can replace no-envy by anonymity and demand-oniliness.

Corollary 4.6 The uniform rule $U^{r}$ is the only rule that satisfies Pareto optimality, strategy-proofness, anonymity, and demand-onliness.

Proof. The uniform rule $U^{r}$ satisfies Pareto optimality, strategy-proofness, anonymity, and demand-onliness.

To prove the remaining part of the corollary let $\varphi$ be a rule that satisfies the properties listed in the corollary. By Lemma 4.9 , it follows that $\varphi$ satisfies equal treatment of equals. Thus, by Corollary $4.5, \varphi=U^{r}$.

The rule $\varphi^{1}$ as defined in Example 4.1 satisfies Pareto optimality, strategy-proofness, and anonymity, but not demand-onliness.

The next property we introduce is a strengthening of demand-onliness. Consider an economy and a "reversal" of this economy, i.e., demanders in the previous economy are 
suppliers with supply equal to their previous demand, non-traders remain non-traders, and suppliers in the previous economy are demanders with demand equal to their previous supply. Reversibility states that in such a reversed economy, the allotment changes in one economy equal the reversed allotment changes of the other economy.

Reversibility: For all $N \in \mathcal{N}$, all $e=(R, \omega) \in \mathcal{E}^{N}$, and all $\bar{e}=(\vec{R}, \bar{\omega}) \in \mathcal{E}^{N}$, if for all $i \in N, d_{i}(e)=-d_{i}(\bar{e})$, then for all $i \in N, \Delta \varphi_{i}(e)=-\Delta \varphi_{i}(\bar{e})$.

Clearly, by applying reversibility two times we obtain demand-onliness.

It is easy to check, that the uniform rule $U^{r}$ and the proportional rule $\operatorname{Pr}^{r}$ satisfy reversibility.

Theorem 4.2 The uniform rule $U^{r}$ is the only rule that satisfies Pareto optimality, equal treatment of equals, endowment strategy-proofness, and reversibility.

Proof. As shown in Lemma 4.7, the uniform rule $U^{r}$ satisfies endowment strategyproofness. Furthermore, $U^{r}$ satisfies Pareto optimality, equal treatment of equals, and reversibility.

To prove the remaining part of the theorem, let $\varphi$ be a rule that satisfies the properties listed in the theorem.

Let $N \in \mathcal{N}, e=(R, \omega) \in \mathcal{E}^{N}$, and suppose, by contradiction, that $\varphi(e) \neq U^{r}(e)$. Since $\varphi$ is Pareto optimal it follows that either $z(e)>0$ or $z(e)<0$. By reversibility, without loss of generality, we can assume that $z(e)>0$. By demand-onliness, without loss of generality, we can assume that for all $i, j \in N, p\left(R_{i}\right)=p\left(R_{j}\right)$ and for all $i \in N$, $2 p\left(R_{i}\right)-w_{i} \geq 0$. By Corollary 4.3 , it follows that

$$
\begin{aligned}
& \text { for all } i \in D(e), \omega_{i} \leq \varphi_{i}(e) \leq p\left(R_{i}\right) \text { and } \\
& \text { for all } i \notin D(e), \varphi_{i}(e)=p\left(R_{i}\right)=U_{i}^{\tau}(e) .
\end{aligned}
$$

We denote the set of demanders with zero as individual endowments by $O(e)=\{i \epsilon$ $\left.D(e) \mid \omega_{i}=0\right\}$.

Case 1: $\quad O(e)=D(e)$. Then, for all $i, j \in D(e), d_{i}(e)=d_{j}(e)$. By $(4.5)$ and equal treatment of equals, $\varphi(e)=U^{r}(e)$. This is a contradiction.

Case 2: $\quad O(e) \subseteq D(e)$. By (4.5) and equal treatment of equals, it follows that for all $i, j \in O(e), \Delta \varphi_{i}(e)=\Delta \varphi_{j}(e)$. Since $\varphi(e) \neq U^{\prime \prime}(e)$, there exists $k \in D(e) \backslash O(e)$ such that $\Delta \varphi_{k}(e) \neq \Delta U_{k}^{r}(e)$. We consider the following two subcases.

Case 2.1: $\Delta \varphi_{k}(e)<\Delta U_{k}^{r}(e)$. Similar to the proof of Corollary 4.3 define $\omega(k) \in \mathbb{R}_{+}^{N}$ by $\omega(k)_{k}=0$ and $\omega(k)_{i}=\omega_{i}$ for all $i \in N \backslash\{k\}$. Let $e(k)=(R, \omega(k)) \in \mathcal{E}^{N}$. Then, by Lemma $4.8, \Delta \varphi_{k}(e) \geq \Delta \varphi_{k}(e(k)) \geq 0$. Furthermore, it follows that $\Delta U_{k}^{r}(e(k)) \geq$ $\Delta U_{k}^{r}(e)$. Hence, $\Delta U_{k}^{r}(e(k))>\Delta \varphi_{k}(e(k))$.

Case 2.2: $\Delta \varphi_{k}(e)>\Delta U_{k}^{r}(e)$. Let $e(k)$ be as in Case 2.1. Then, since $U_{k}^{r}(e)<p\left(R_{k}\right)$, $\Delta U_{k}^{r}(e(k))=\Delta U_{k}^{r}(e)$. Let $\bar{e}=(R, \bar{\omega}) \in \mathcal{E}^{N}$ and $\bar{e}(k)=(R, \bar{\omega}(k)) \in \mathcal{E}^{N}$ be such that for all $i \in N, d_{i}(e)=-d_{i}(\bar{e})$ and $d_{i}(e(k))=-d_{i}(\bar{e}(k))$. Then, by reversibility, it follows for all $i \in N$ that $\Delta \varphi_{i}(e)=-\Delta \varphi_{i}(\bar{e})$ and $\Delta \varphi_{i}(e(k))=-\Delta \varphi_{i}(\bar{e}(k))$. If $\varphi_{k}(\bar{e}(k))=p\left(R_{k}\right)$, 
then $\varphi_{k}(e(k))=p\left(R_{k}\right)$ and clearly $\Delta \varphi_{k}(e(k)) \geq \Delta \varphi_{k}(e)$. If $\varphi_{k}(e(k)) \neq p\left(R_{k}\right)$, since $z(\bar{e})<0, z(\bar{e}(k))<0$. Furthermore, $\bar{\omega}$ is a $k$-deviation from $\bar{\omega}(k)$ by withholding and Lemma 4.8 implies $\Delta \varphi_{k}(\bar{e}) \geq \Delta \varphi_{k}(\bar{e}(k))$. Hence, by reversibility, $\Delta \varphi_{k}(e(k)) \geq \Delta \varphi_{k}(e)$ and $\Delta \varphi_{k}(e(k))>\Delta U_{k}^{r}(e(k))$.

Thus, for the economy $e(k)$ it follows that $\varphi_{j}(e(k)) \neq U_{j}^{r}(e(k))$. Note that the economy $e(k)$ has one demanders with zero as individual endowment more than $e$, i.e., $|O(e(k))|=|O(e)|+1$. By Case $1, O(e(k)) \subsetneq=D(e(k))$.

Similarly, proceeding from the economy $e(k)$, we consider a unilateral change of an individual endowment by withholding such that the set of demanders with individual endowment zero increases by one and the uniform reallocation for this economy is un-

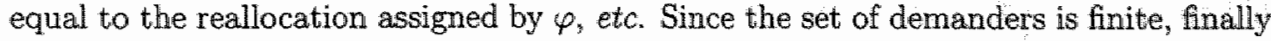
we end up in an economy $e^{\prime}$ with $D\left(e^{\prime}\right)=O\left(e^{\prime}\right)$ and $\varphi\left(e^{\prime}\right) \neq U^{r}\left(e^{\prime}\right)$. By Case 1 , this is a contradiction.

Next, we consider the well-known property of peak-onliness: the rule only depends on the peaks instead of the complete profile (see also Section 2.2, page 22).

Peak-onliness: For all $N \in \mathcal{N}$, all $e=(R, \omega) \in \mathcal{E}^{N}$, and all $\bar{e}=(\bar{R}, \omega) \in \mathcal{E}^{N}$, if $p(R)=p(\bar{R})$, then $\varphi(e)=\varphi(\bar{e})$.

Many well-known rules are peak-only. The uniform allocation rule, the proportional reallocation rule, and the uniform reallocation rule are peak-only. Clearly, if a rule takes "intensities" or the shape of the preferences into account, then one would expect that it is apt to be vulnerable to strategic behavior. The following theorem shows that in Theorem 4.1 we can replace strategy-proofness by peak-onliness.

This characterization of the uniform reallocation rule can be interpreted as an extension of Thomson's characterization of the uniform allocation rule by Pareto optimality, no-envy, and peak-onliness (see Theorem 2.3). The proofs of Theorem 2.3 and the following Theorem 4.3 are simillar. ${ }^{14}$

Theorem 4.3 The uniform rule $U^{r}$ is the only rule that satisfies Pareto optimality, no-envy, and peak-onliness.

Proof. By same-sidedness and Lemma 4.6 it follows that the uniform rule $U^{r}$ satisfies Pareto optimality and no-envy. Peak-onliness of $U^{r}$ follows from its definition.

To prove the remaining part of the theorem let $\varphi$ be a rule that satisfies the properties listed in the theorem.

Let $N \in \mathcal{N}, e=(R, \omega) \in \mathcal{E}^{N}$, and suppose, by contradiction, that $\varphi(e) \neq U^{r}(e)$. Since $\varphi$ is Pareto optimal it follows that either $z(e)>0$ or $z(e)<0$.

Case 1: $\quad z(e)<0$. Since $\varphi(e) \neq U^{r}(e)$, by Lemma 4.1 , there exist agents $j, k \in N$ such that $p\left(R_{j}\right)\left\langle\varphi_{j}(e)\right.$ and $\left.\Delta \varphi_{j}(e)\right\rangle \Delta \varphi_{k}(e)$. Hence, by no-envy, $\left.\varphi_{j}(e)=\omega_{j}+\Delta \varphi_{j}(e)\right\rangle$ $p\left(R_{j}\right)>\left(\omega_{j}+\Delta \varphi_{k}(e)\right)^{+}$. Let $\bar{e}=(\vec{R}, \omega) \in \mathcal{E}^{N}$, be such that $\vec{R}$ is a $j$-deviation from

\footnotetext{
${ }^{14}$ This result was independently achieved by Moreno (1996), Theorem 1 .
} 
$R$ with $p\left(\bar{R}_{j}\right)=p\left(R_{j}\right)$ and $\left(\omega_{j}+\Delta \varphi_{k}(e)\right)^{+} \bar{P}_{j} \varphi_{j}(e)$. By peak-onliness it follows that $\varphi(\bar{e})=\varphi(e)$ and particularly that $\Delta \varphi_{k}(e)=\Delta \varphi_{k}(\bar{e})$. Hence, for the economy $\bar{e}$ and agent $j_{1}\left(\omega_{j}+\Delta \varphi_{k}(\bar{e})\right)+\bar{P}_{j} \varphi_{j}(\bar{e})$. This is a contradiction to no-envy.

Case 2: $z(e)>0$. Similar to Case 1 .

In the proof of Theorem 4.3 we prove, without loss of generality, that for an economy with excess supply the rule $\varphi$ assigns the uniform reallocation. The proof for the excess demand case is very similar. As we state in the next corollary in this case we can weaken no-envy to weak no-envy.

Corollary 4.7 Let $\varphi$ be a rule that satisfies Pareto optimality, weak no-envy, and peak-onliness. Then, for all $N \in \mathcal{N}$ and all $e=(R, \omega) \in \mathcal{E}^{N}$ such that $z(e) \geq 0$, $\varphi(e)=U^{r}(e)$.

Proof. Similar to the proof of Corollary 4.1 , it is sufficient to adjust the proof of Theorem 4.3 to the excess demand case. Since the lower bound zero is not active (see Remark 4.2) in this case, instead of no-envy we can apply weak no-envy.

It is easy to check that the rule $\varphi^{1}$ as defined in Example 4.1 satisfies peak-onliness. Hence, Example 4.1 shows that it is not possible to weaken no-envy in Theorem 4.3 to weak no-envy. However, as we show in the next corollary, we can weaken no-envy in Theorem 4.3 to weak no-envy if, at the same time, we strengthen peak-onliness to demand-onliness.

Corollary 4.8 The uniform rule $U^{r}$ is the only rule that satisfies Pareto optimality, weak no-envy, and demand-onliness.

Proof. The uniform rule $U^{r}$ satisfies Pareto optimality, weak no-envy, and demandonliness.

To prove the remaining part of the corollary let $\varphi$ be a rule that satisfies the properties listed in the corollary. By Pareto optimality, peak-onliness, and Corollary 4.7, it follows that for all $N \in \mathcal{N}$ and $e=(R, \omega) \in \mathcal{E}^{N}$ such that $z(e) \geq 0$, $\varphi(e)=U^{r}(e)$. It remains to prove that for all $N \in \mathcal{N}$ and $e=(R, \omega) \in \mathcal{E}^{N}$ such that $z(e)<0, \varphi(e)=U^{r}(e)$. By demand-onliness it is sufficient to consider $N \in \mathcal{N}$ and $e=(R, \omega) \in \mathcal{E}^{N}$ such that for all $i, j \in N, \omega_{i}=\omega_{j}$. Now, the remainder of the proof is similar to the proof of Theorem 4.3 by applying weak no-envy instead of no-envy.

The rule $\varphi^{1}$ as defined in Example 4.1 satisfies Pareto optimality, weak no-envy, but not demand-onliness.

A summary of the characterizations of the uniform rule $U^{r}$ by Pareto optimality, strategy-proofness properties, equity properties, etc., can be found in Table 4.1. For a comparison with corresponding characterizations of the uniform allocation rule $U$ we refer to Table 2.1. 


\begin{tabular}{|l|c|c|c|c|c|c|c|c|c|c|}
\hline & $\begin{array}{c}\text { Th } \\
4.1\end{array}$ & $\begin{array}{c}\text { Co } \\
\text { Co }\end{array}$ & 4.2 & 4.4 & 4.5 & 4.6 & 4.2 & 4.3 & 4.7 & 4.8 \\
\hline \hline for all $e \in \mathcal{E}^{N}$ & $\times$ & & & & $\times$ & $\times$ & $\times$ & $\times$ & & $\times$ \\
\hline $\begin{array}{l}\text { not for } e \in \mathcal{E}^{N} \\
\text { with } z(e)<0\end{array}$ & & $\times$ & $\times$ & $\times$ & & & & & $\times$ & \\
\hline \hline Pareto optimality & $\times$ & $\times$ & $\times$ & $\times$ & $\times$ & $\times$ & $\times$ & $\times$ & $\times$ & $\times$ \\
\hline strategy-proofness & $\times$ & $\times$ & $\times$ & & $\times$ & $\times$ & & & & \\
\hline $\begin{array}{l}\text { endowment } \\
\text { strategy-proofness }\end{array}$ & & & & $\times$ & & & $\times$ & & & \\
\hline no-envy & $\times$ & & & & & & & $\times$ & & \\
\hline wreak no-envy & & $\times$ & & $\times$ & & & & & $\times$ & $\times$ \\
\hline $\begin{array}{l}\text { equal treatment } \\
\text { of equals }\end{array}$ & & & $\times$ & & $\times$ & & $\times$ & & & \\
\hline $\begin{array}{l}\text { anonymity } \\
\text { demand-onliness }\end{array}$ & & & & & & $\times$ & & & & \\
\hline reversibility & & & & & & & $\times$ & & & \\
\hline peak-onliness & & & & & & & & $\times$ & $\times$ & \\
\hline
\end{tabular}

Table 4.1: Characterizations of the uniform rule by Pareto optimality, strategyproofness properties, equity properties, etc.

\subsection{Solidarity properties}

Next we analyze some "solidarity properties" similar to those we introduced in Chapter 2. As already explained in Chapter 2, a solidarity property describes the effect of certain changes in a single parameter of the economy while the other parameters are kept fixed, e.g., the population, the preferences, or the individual endowments. If after such a change, either all agents that are not responsible for the change (weakly) lose together or all (weakly) gain together, then depending on which parameter has been changed, the rule satisfies population-monotonicity, welfare-domination under preference-replacement, or endowment-monotonicity. Thomson (1994a, 1995a, 1997a) shows that these properties, when considered for allocation rules, are generally incompatible with Pareto optimality and no-envy. However, as already discussed in Chapter 2 , these incompatibilities do not occur if the change in the parameter is one-sided, i.e., the change does not turn an economy in excess demand into an economy in excess supply, or vice versa. Furthermore, the uniform allocation rule turns out to be essentially the only rule that satisfies any of the one-sided solidarity properties, together with Pareto optimality and no-envy. In this section we explore how the results as achieved for the allocation model carry through to the reallocation model. 


\subsubsection{One-sided population-monotonicity}

First we consider changes in the population while keeping the other parameters fixed. Population-monotonicity requires that after the arrival of new agents either all agents initially present (weakly) lose or all (weakly) gain.

Population-monotonicity: For all $N, \bar{N} \in \mathcal{N}$, all $e=(R, \omega) \in \mathcal{E}^{N}$, and all $\bar{e}=(\tilde{R}, \bar{\omega}) \in \mathcal{E}^{\tilde{N}}$, if $N \subseteq \tilde{N}, R=\tilde{R}_{N}$, and $\omega=\widetilde{\omega}_{N}$, then either [for all $i \in N$, $\varphi_{i}(\bar{e}) R_{i} \varphi_{i}(e)$ ] or [for all $i \in N, \varphi_{i}(e) R_{i} \varphi_{i}(\bar{e})$ ].

Similar to the allocation model, it can be shown that the properties Pareto optimality, no-envy, and population-monotonicity are generally incompatible. See for instance Moreno (1996), Proposition 1. However, the uniform reallocation rule satisfies Pareto optimality, no-envy, and the one-sided version of population-monotonicity, i.e., if the change in the population is such that the change in the initial economy is one-sided, then either all agents initially present (weakly) lose or all (weakly) gain. Here, we introduce a weaker one-sided version of population-monotonicity.

One-sided population-monotonicity states that if two disjoint economies are merged, either both of excess demand or both of excess supply, then all agents of one of the economies (weakly) lose or all (weakly) gain (see also Section 2.2, page 24). Before we give a formal definition, we introduce some notation.

Let $N, \bar{N} \in \mathcal{N}, e=(R, \omega) \in \mathcal{E}^{N}, \bar{e}=(\bar{R}, \bar{\omega}) \in \mathcal{E}^{\bar{N}}$, and $N \cap \bar{N}=\emptyset$. Then, $R \cup \bar{R}=(R, \vec{R}) \in \mathcal{R}^{N \cup N}$ denotes the preference profile that consists of the preferences of the agents in $N \cup \bar{N}$. Similarly, $\omega \cup \bar{\omega}=(\omega, \bar{\omega}) \in \mathbb{R}_{+}^{N \cup \bar{N}}$ denotes the endowment vector that consists of the individual endowments of the agents in $N \cup \vec{N}$. Then, the merged economy $(R \cup \bar{R}, \omega \cup \bar{\omega}) \in \mathcal{E}^{N \cup \bar{N}}$ is denoted by $e \cup \bar{e}$.

One-sided population-monotonicity: For all $N, \bar{N} \in \mathcal{N}$, all $e=(R, \omega) \in \mathcal{E}^{N}$, and all $\bar{e}=(\bar{R}, \bar{\omega}) \in \mathcal{E}^{N}$, if $N \cap \bar{N}=\emptyset$ and $z(e) \cdot z(\bar{e})>0$, then either [for all $i \in N$, $\left.\varphi_{i}(e \cup \tilde{e}) R_{i} \varphi_{i}(e)\right]$ or [for all $\left.i \in N, \varphi_{i}(e) R_{i} \varphi_{i}(e \cup \bar{e})\right]$.

Note that $z(e) \cdot z(\vec{e})>0$ if, and only if, both problems have excess demand or both problems have excess supply.

Lemma 4.10 The uniform rule $U^{r}$ satisfies one-sided population monotonicity.

Proof. Let $N, \bar{N} \in \mathcal{N}, e=(R, \omega) \in \mathcal{E}^{N}$, and $\bar{\varepsilon}=(\bar{R}, \bar{\omega}) \in \mathcal{E}^{\bar{N}}$ be such that $N \cap \bar{N}=\emptyset$ and $z(e)-z(\vec{e})>0$. In order to prove one-sided population monotonicity we have to show that either (i) [for all $i \in N, \varphi_{i}(e \cup \bar{e}) R_{i} \varphi_{i}(e)$ ] or (ii) [for all $i \in N, \varphi_{i}(e) R_{i} \varphi_{i}(e \cup \bar{e})$ ].

Case 1: $z(e), z(\bar{e})<0$. Let $e \cup \bar{e}=\left(R^{\prime}, \omega^{\prime}\right)$. Let $\lambda, \bar{\lambda}$ and $\mu$ be the minimal allotment changes of $U^{r}$ for the economies $e$, $\bar{e}$, and $e \cup \bar{e}, i . e$, for all $i \in N, U_{i}^{r}(e)=$ $\max \left\{p\left(R_{i}\right), \omega_{i}+\lambda\right\}$, for all $i \in \bar{N}, U_{i}^{r}(\bar{e})=\max \left\{p\left(\bar{R}_{i}\right), \bar{\omega}_{i}+\bar{\lambda}\right\}$, and for all $i \in N \cup \bar{N}$, $U_{i}^{r}(e \cup \bar{e})=\max \left\{p\left(R_{i}^{\prime}\right), \omega_{i}^{\prime}+\mu\right\}$. Suppose, by contradiction, that there exist agents $j, k \in N$ such that $j$ is strictly better off at $e \cup \vec{e}$ than at $e$ and for $k$ the converse holds. 
This can only hold if $j, k \in S(e)$. Hence, $j$ is not satiated at e and $k$ is not satiated at eUe. Hence, $p\left(R_{j}\right) \leq U_{j}^{r}(e \cup \bar{e})<U_{j}^{r}(e)=\omega_{j}+\lambda$ and $p\left(R_{k}\right) \leq U_{k}^{r}(e)<U_{k}^{r}(e U \bar{e})=\omega_{k}+\mu$. So, for the corresponding allotment changes it holds that $\Delta U_{j}^{r}(e)=\lambda>\Delta U_{j}^{r}(e \cup \bar{e}) \geq \mu$ and $\Delta U_{k}^{r}(e \cup \bar{e})=\mu>\Delta U_{k}^{r}(e) \geq \lambda$. This is a contradiction. Hence, either (i) or (ii) must hold.

Case 2: $z(e), z(\bar{e})>0$. Similar to Case 1.

Note that the proportional rule $P_{r^{r}}$ and the uniform rule $U^{r}$ also satisfy the somewhat stronger one-sided population monotonicity conditions as considered in Moreno (1996) and Thomson (1995d) ${ }^{15}$

Next, we show that one-sided population-monotonicity together with Pareto optimality and weak no-envy imply demand-onliness.

Lemma 4.11 Let $\varphi$ satisfy Pareto optimality, weak no-envy; and one-sided populationmonotonicity. Then, $\varphi$ satisfies demand-onliness.

Proof. Let $\varphi$ be a rule that satisfies Pareto optimality, weak no-envy, and one-sided population-monotonicity. Let $N \in \mathcal{N}, e=(R, \omega) \in \mathcal{E}^{N}$, and $\bar{e}=(\bar{R}, \bar{\omega}) \in \mathcal{E}^{N}$ be such that for all $i \in N, d_{i}(e)=d_{i}(\bar{e})$. In order to prove demand-onliness we have to show that for all $i \in N, \Delta \varphi_{i}(e)=\Delta \varphi_{i}(\bar{e})$.

Case 1: $z(e) \leq 0$. Assume, without loss of generality, that $N=\{1,2, \ldots, n\}$. Let $N^{\prime}=\{n+1, \ldots, 2 n\}$ and define $e^{\prime}=\left(R^{\prime}, \omega^{\prime}\right) \in \mathcal{E}^{N^{\prime}}$ such that for all $i \in N, \omega_{i+\pi}^{\prime}=\bar{\omega}_{i}$ and $p\left(R_{i+n}^{i}\right)=p\left(\bar{R}_{i}\right)$. Clearly, $z\left(e^{\prime}\right)<0$. Hence, $N \cap \bar{N}=\emptyset$ and $z(e) \cdot z\left(e^{\prime}\right)>0$. Weak no-envy and same-sidedness imply for all $i \in N$,

$$
\Delta \varphi_{i}\left(e \cup e^{\prime}\right)=\Delta \varphi_{i+n}\left(e \cup e^{\prime}\right)
$$

By one-sided population-monotonicity and same-sidedness,

(i) for all $i \in N, \Delta \varphi_{i}(e) \leq \Delta \varphi_{i}\left(e \cup e^{\prime}\right)$ or

(ii) for all $i \in N, \Delta \varphi_{i}(e) \geq \triangle \varphi_{i}\left(e \cup e^{\prime}\right)$,

and

(iii) for all $i \in N, \Delta \varphi_{i+n}(e) \leq \Delta \varphi_{i+n}\left(e \cup e^{\prime}\right)$ or

(iv) for all $i \in N, \Delta \varphi_{i+n}(e) \geq \Delta \varphi_{i+n}\left(e \cup e^{\prime}\right)$.

By same-sidedness and weak no-envy it follows that either [(i) and (iii)] or [(ii) and (iv)]. By feasibility, this can only hold if for all $i \in N, \Delta \varphi_{i}(e)=\Delta \varphi_{i}\left(e \cup e^{\prime}\right)$. Similarly it follows that for all $i \in N^{\prime}, \Delta \varphi_{i}\left(e^{\prime}\right)=\Delta \varphi_{i}\left(e \cup e^{\prime}\right)$. Hence, by (4.6), for all $i \in N$, $\Delta \varphi_{i}(e)=\Delta \varphi_{i+n}\left(e^{\prime}\right)$. By applying the same argument to the economies $\vec{e}$ and $e^{\prime}$ instead

${ }^{15}$ The following definition of one-sided population-monotonicity is due to Moreno (1996): For all $N, N^{r} \in \mathcal{N}$ with $N^{\prime} \subset \mathcal{N}$, for all $e=\left(R_{v} \omega\right) \in \mathcal{R}^{N} \times \mathbb{R}_{+}^{N}$, if either (i) $\sum_{N} p\left(R_{i}\right) \leq \sum_{N} w_{i}$ and $\sum_{N^{\prime}} p\left(R_{i}\right) \leq \sum_{N^{j} \omega_{i}}$ or (ii) $\sum_{N^{\prime}} p\left(R_{i}\right) \geq \sum_{N^{\prime}} \omega_{i}$ and $\sum_{N^{\prime}} p\left(R_{i}\right) \geq \sum_{N^{\prime}} \omega_{i}$, then for all $i \in N^{\prime}$, $\varphi_{i}(e) R_{i} \varphi_{i}\left(e^{t}\right)$, where $e^{\prime}=\left(R_{N^{\prime}, \omega^{*}}\right)$, or for all $i \in N^{*}, \varphi_{i}\left(e^{\prime}\right) R_{i} \varphi_{i}(e)$, 
of $e$ and $e^{\prime}$ it followis that for all $i \in N, \Delta \varphi_{i}(\tilde{e})=\Delta \varphi_{i+n}\left(e^{\prime}\right)$. Hence, for all $i \in N$, $\Delta \varphi_{i}(e)=\Delta \varphi_{i+n}\left(e^{\prime}\right)=\Delta \varphi_{i}(\vec{e})$.

Case 2: $z(e)>0$. Similar to Case 1 .

Thomson (Theorem 2.6) shows that the uniform allocation rule is the only rule that satisfies Pareto optimality, no-envy, one-sided population-monotonicity, and replicationinvariance. Here, we show that the uniform reallocation rule is the only reallocation rule that satisfies Pareto optimality, weak no-envy, and one-sided population-monotonicity. So, in the following "reallocation version" of Theorem 2.6 , replication-invariance can be omitted. Moreno (1996) independently proved this theorem. However, he applied a somewhat stronger notion of one-sided population-monotonicity and another proof technique.

Theorem 4.4 ${ }^{16}$ The uniform rule $U^{r}$ is the only rule that satisfies Pareto optimality, weak no-envy, and one-sided population monotonicity.

Proof. By same-sidedness and Lemmas 4.6 and 4.10 it follows that the uniform rule $U^{r}$ satisfies Pareto optimality, weak no-envy, and one-sided population monotonicity.

To prove the remaining part of the theorem let $\varphi$ be a rule that satisfies the properties listed in the theorem.

Then, by Lemma 4.11, $\varphi$ satisfies demand-onliness. Hence, by Corollary $4.8, \varphi=U^{r}$.

For an alternative proof of Theorem 4.4 we refer the interested reader to Moreno (1996), proof of Theorem 2.

\subsubsection{One-sided replacement-domination}

Next, we consider a change of one agent's preference relation, keeping the set of agents, the preferences of the remaining agents, and the individual endowments fixed. Welfare-domination under preference-replacement requires that either all remaining agents (weakly) lose or all (weakly) gain.

Welfare-domination under preference-replacement, or replacement-domination: For all $N \in \mathcal{N}$, all $e=(R, \omega) \in \mathcal{E}^{N}$, all $\bar{e}=(\bar{R}, \omega) \in \mathcal{E}^{N}$, and all $j \in N$, if $\bar{R}$ is a $j$-deviation from $R$, then either [for all $i \in N \backslash\{j\}, \varphi_{i}(e) R_{i} \varphi_{i}(\bar{e})$ ] or [for all $i \in N \backslash\{j\}$, $\left.\varphi_{i}(\bar{e}) R_{i} \varphi_{i}(e)\right]$.

In the sequel, we use the shorter phrase of replacement-domination for welfaredomination under preference-replacement.

\footnotetext{
${ }^{16}$ Theorem 4.4 is a strengthening of a result of Klaus, Peters, and Storcken (1997b, Theorem 3.2): the uniform rule $U^{r}$ is the only rule that satisfies Pareto optimality, no-envy; and one-sided populationmonotonicity.
} 
Similar to the allocation model, it can be shown that the properties Pareto optimality, weak no-envy, and replacement-domination are generally incompatible. See for instance Thomson (1995d), Proposition 3. However, the uniform reallocation rule satisfies Pareto optimality, no-envy, and the one-sided version of replacement-domination, i.e., if the change of one agent's preference relation is one-sided, then either all remaining agents (weakly) lose or all (weakly) gain (see also Section 2.2, page 24).

One-sided welfare-domination under preference-replacement, or replacementdomination: For all $N \in \mathcal{N}$, all $e=(R, \omega) \in \mathcal{E}^{N}$, all $\bar{e}=(\vec{R}, \omega) \in \mathcal{E}^{N}$, and all $j \in N$, if $\bar{R}$ is a $j$-deviation from $R$ and $\bar{e}$ is a one-sided change of $e$, then either [for all $i \in N \backslash\{j\}, \varphi_{i}(e) R_{i} \varphi_{i}(\bar{e})$ ] or [for all $\left.i \in N \backslash\{j\}, \varphi_{i}(\bar{e}) R_{i} \varphi_{i}(e)\right]$.

Lemma 4.12 The uniform rule $U^{\text {r }}$ satisfies one-sided replacement-domination.

Proof. Let $N \in \mathcal{N}, e=(R, \omega) \in \mathcal{E}^{N}$, and $\bar{e}=(\bar{R}, \omega) \in \mathcal{E}^{N}$ be such that $\bar{R}$ is a $j$-deviation from $R$ and $\bar{e}$ is a one-sided change of $e$. In order to prove onesided replacement-domination, we have to show that either (i) [for all $i \in N \backslash\{j\}$, $U_{i}^{r}(e) R_{i} U_{i}^{r}(\bar{e})$ ] or (ii) [for all $\left.i \in N \backslash\{j\}, U_{i}^{r}(\bar{e}) R_{i} U_{i}^{r}(e)\right]$.

Case 1: $\quad z(e) \leq 0$ and $z(\bar{e}) \leq 0$. Hence, for all $i \in N, U_{i}^{r}(e)=\max \left\{p\left(R_{i}\right)\right.$, $\left.\omega_{i}+\lambda\right\}$, where $\bar{\lambda}$ solves $\sum_{N} U_{i}^{r}(e)=\sum_{N} \omega_{i}$ and $U_{i}^{r}(\bar{e})=\max \left\{p\left(\bar{R}_{i}\right), \omega_{i}+\bar{\lambda}\right\}$, where $\bar{\lambda}$ solves $\sum_{N} U_{i}^{r}(\bar{e})=\sum_{N} \omega_{i}$. If $U_{j}^{r}(e)=U_{j}^{r}(\bar{e})$, then for all $i \in N \backslash\{j\}, U_{i}^{r}(e)=U_{i}^{r}(\bar{e})$. Otherwise ${ }_{\text {we }}$ distinguish two cases.

Case 1.1: $U_{j}^{r}(e)<U_{j}^{r}(\bar{e})$. Thus, $p\left(\bar{R}_{j}\right)>p\left(R_{j}\right)$. It follows that $\bar{\lambda} \leq \lambda$. Hence, for all $i \in N \backslash\{j\}, U_{i}^{r}(e)=\max \left\{p\left(R_{i}\right), \omega_{i}+\lambda\right\} \geq \max \left\{p\left(\bar{R}_{i}\right), \omega_{i}+\bar{\lambda}\right\}=U_{i}^{r}(\bar{e}) \geq p\left(\bar{R}_{i}\right)$. This implies (ii).

Case 1.2: $\quad U_{j}^{r}(e)>U_{j}^{r}(\bar{e})$. Thus, $p\left(\bar{R}_{j}\right)<p\left(R_{j}\right)$. It follows that $\bar{\lambda} \geq \lambda$. Hence, for all

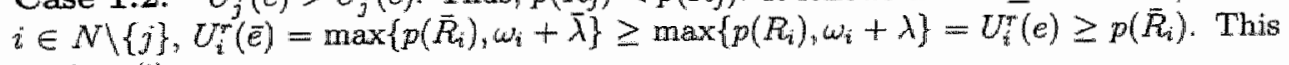
implies (i).

Case 2: $z(e) \geq 0$ and $z(\bar{e}) \geq 0$. Similar to Case 1 .

Next, we show that the uniform rule $U^{r}$ is the only rule that satisfies Pareto optimality, no-envy, one-sided replacement-domination and the following well-known property of replication-invariance.

Replication-invariance states that if an economy is replicated, i.e., the preference profile and the individual endowments are replicated, then also the allocation assigned by the rule must be replicated (see also Section 2.2 , page 25 ). In order to formally describe a replicated problem, we introduce the following notation.

Let $k \in \mathbb{N}$ and $\bar{N}, N_{1}, \ldots, N_{k} \in \mathcal{N}$. Then, the collection $\left\{N_{1}, \ldots, N_{k}\right\}$ constitutes a partition of $\bar{N}$ if $\bar{N}=\bigcup_{l \in\{1, \ldots k\}} N_{l}$ and $N_{l} \cap N_{m}=\emptyset$ for all $l, m \in\{1, \ldots k\}, l \neq m$.

Let $N, \bar{N} \in \mathcal{N}, e=(R, \omega) \in \mathcal{E}^{N}$, and $\tilde{e}=(\vec{R}, \bar{\omega}) \in \mathcal{E}^{\bar{N}}$ be such that

(i) there exist $k \in \mathbb{N}$ and $N_{1}, \ldots, N_{k} \in \mathcal{N}$ such that $N=N_{1}$ and $\left\{N_{1}, \ldots, N_{k}\right\}$ constitutes a partition of $\bar{N}$, 
(ii) there exist $k$ bijective functions, indexed by $l \in\{1, \ldots, k\}, b_{l}: N \rightarrow N_{l}$, such that for all $i \in N, R_{i}=\vec{R}_{b_{i}(i)}$ and $\omega_{i}=\bar{\omega}_{b_{i}(i)}{ }^{17}$

Then, $\tilde{e}$ is a $k$-replica of $e$. Let $\bar{e} \in \mathcal{E}^{N}$ be a $k$-replica of $e \in \mathcal{E}^{N}$ and let $x \in \mathbb{R}_{+}^{N}$. If $\dot{\vec{x}} \in \mathbb{R}_{+}^{N}$ is such that $x_{i}=\bar{x}_{b_{l}(i)}$ for the functions $b_{l}, l \in\left\{1, \ldots k_{k}\right\}$, as described in (ii), then $\vec{x}$ a-replica of $x$.

Replication-invariance: For all $N, \bar{N} \in \mathcal{N}$, all $e=(R, \omega) \in \mathcal{E}^{N}$, and all $\bar{e}=$ $(\bar{R}, \bar{\omega}) \in \mathcal{E}^{\bar{N}}$, if $\bar{e}$ is a $k$-replica of $e_{\text {, then }} \varphi(\bar{e})$ is a $k$-replica of $\varphi(e)$.

It is easy to check, that the uniform rule $U^{r}$ and the proportional rule $\operatorname{Pr}^{r}$ satisfy replication-invariance.

For the allocation model, Thomson establishes a characterization of the uniform allocation rule by Pareto optimality, no-envy, one-sided replacement-domination, and replication-invariance (see Theorem 2.7).

Before we prove the "reallocation version" of Theorem 2.7, we show that Pareto optimality, no-envy, one-sided replacement-domination, and replication-invariance imply peak-onliness.

Lenma 4.13 Let $\varphi$ satisfy Pareto optimality, no-envy, one-sided replacement-domination, and replication-invariance. Then, $\varphi$ satisfies peak-onliness.

Proof. Let $\varphi$ be a rule that satisfies Pareto optimality, no-envy, one-sided replacementdomination, and replication-invariance. Let $N \in \mathcal{N}_{n} e=(R, \omega) \in \mathcal{E}^{N}$, and $\bar{e}=(\bar{R}, \omega) \in$ $\mathcal{E}^{N}$ be such that $p(R)=p(\bar{R})$. In order to prove peak-onliness we have to show that $\varphi(e)=\varphi(\bar{e})$.

Case 1: $z(\epsilon) \leq 0$. Assume, without loss of generality, that $N=\{1,2, \ldots, n\}$. Let $N^{*}=\{n+1, \ldots, 2 n\}$ and define $e^{0}=\left(R^{0}, \omega^{0}\right) \in \mathcal{E}^{N \cup N^{\prime}}$ such that for all $i \in N$, $\omega_{i}=\omega_{i}^{0}=\omega_{n+i}^{0}$ and $R_{i}=R_{i}^{0}=R_{i+n}^{0}$. Clearly, $e^{0}$ is a 2-replica of $e$. Hence, by replication-invariance, for all $i \in N, \varphi_{i}(e)=\varphi_{i}\left(e^{0}\right)=\varphi_{n+i}\left(e^{0}\right)$. Next, we change the

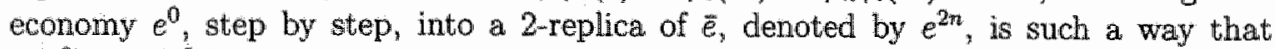
$\varphi\left(e^{0}\right)=\varphi\left(e^{2 n}\right)$. By replication-invariance, this completes the proof.

Step 1: Let $R^{\mathbb{1}} \in \mathcal{R}^{N u N^{\prime \prime}}$ be a 1-deviation from $R^{0}$ such that $R_{1}^{1}=\ddot{R}_{\mathbb{1}}$. Let $e^{\mathbb{1}}=$ $\left(R^{1}, \omega^{0}\right)$ denote this one sided change of economy $e^{0}$. Since $p\left(R_{1}^{1}\right)=p\left(R_{n+1}^{1}\right)$ and $\omega_{1}^{0}=\omega_{n+11}^{0}$, by same-sidedness and no-envy, it follows that $\varphi_{1}\left(e^{1}\right)=\varphi_{n+1}\left(e^{1}\right)$. Suppose that $\varphi_{1}\left(e^{1}\right)<\varphi_{1}\left(e^{0}\right)$. Hence, $\varphi_{n+1}\left(e^{1}\right)<\varphi_{n+1}\left(e^{0}\right)$ and, by same-sidedness and one-sided replacement-domination, for all $i \in N \backslash 1\} \cup N^{\prime}, \varphi_{i}\left(e^{\mathbb{1}}\right) \leq \varphi_{i}\left(e^{0}\right)$. Then, by feasibility, it follows that $\sum_{N \cup N^{\prime}} \omega_{i}^{0}=\sum_{N u N^{\prime}} \varphi_{i}\left(e^{1}\right)<\sum_{N \cup N^{\prime}} \varphi_{i}\left(e^{0}\right)=\sum_{N u N^{\prime}} \omega_{i}^{0}$. This is a contradiction. Similarly, the assumption, $\varphi_{1}\left(e^{1}\right)>\varphi_{1}\left(e^{0}\right)$ yields a contradiction. Hence, $\varphi_{1}\left(e^{1}\right)=\varphi_{1}\left(e^{0}\right)$. Then, by same-sidedness and one-sided replacement-domination, for all $i \in N \backslash\{1\} \cup N^{\prime}, \varphi_{i}\left(e^{1}\right)=\varphi_{i}\left(e^{0}\right)$. Hence, $\varphi\left(e^{1}\right)=\varphi\left(e^{0}\right)$.

\footnotetext{
${ }^{17}$ Let $l \in\{1, \ldots, k\}$ and $i \in N$. Then, aggent $b_{l}(i)$ is the "replica", or the "clone", of agent $i \in N$ in $N_{1}$.
} 
Step 2: Next, let $R^{2} \in \mathcal{R}^{N u N^{*}}$ be a 2-deviation from $R^{1}$ such that $R_{2}^{2}=\bar{R}_{2}$. Let $e^{2}=\left(R^{2}, \omega^{0}\right)$ denote this one-sided change of economy $e^{1}$. Similarly as before we deduce that $\varphi\left(e^{2}\right)=\varphi\left(e^{1}\right)=\varphi\left(e^{0}\right)$.

Steps $k=3, \ldots, n$ : For all $k \in\{1,2, \ldots, n\}$ we let $R^{k} \in \mathcal{R}^{N U N^{\prime}}$ be a $k$-deviation from $R^{k-1}$ such that $R_{k}^{k}=\tilde{R}_{k}$ and $e^{k}=\left(R^{k}, \omega^{0}\right)$. Applying the argument of the previous step, it follows that for all $k \in\{1,2, \ldots, n\}, \varphi\left(e^{k}\right)=\varphi\left(e^{k-1}\right)=\varphi\left(e^{0}\right)$.

Step $n+1$ : Next, let $R^{n+1} \in \mathcal{R}^{N \cup N^{\prime}}$ be a $(n+1)$-deviation from $R^{n}$ such that $R_{n+1}^{2}=$ $\bar{R}_{1}$. Let $e^{n+1}=\left(R^{n+1}, \omega^{0}\right)$ denote this one-sided change of economy $e^{n}$. Similarly as before we deduce that $\varphi\left(e^{r}\right)=\varphi\left(e^{0}\right)$.

Steps $k=n+2, \ldots, 2 n$ : For all $k \in\{n, \ldots, 2 n\}$ we let $R^{k} \in \mathcal{R}^{N \cup N^{\prime}}$ be a $k$-deviation from $R^{k-1}$ such that $R_{k}^{k}=\vec{R}_{k}$ and $e^{k}=\left(R^{k}, \omega^{0}\right)$. Similarly as before, it follows for all $k \in\{n, \ldots, 2 n\}, \varphi\left(e^{k}\right)=\varphi\left(e^{k-1}\right)=\varphi\left(e^{0}\right)$.

Hence, by construction, $e^{2 n}$ is a 2-replica of $\bar{e}$ and $\varphi\left(e^{0}\right)=\varphi\left(e^{2 n}\right)$. By replicationinvariance, this implies $\varphi(e)=\varphi(\bar{e})$.

Case 2: $z(e)>0$. Similar to Case 1 .

Theorem 4.5 The uniform rule $U^{r}$ is the only rule that satisfies Pareto optimality, no-envy, one-sided replacement-domination, and replication-invariance.

Proof. By same-sidedness and Lemmas 4.6 and 4.12 it follows that the uniform rule $U^{r}$ satisfies Pareto optimality, no-envy, and one-sided replacement-domination. Furthermore, the uniform rule $U^{r}$ is replication-invariant.

To prove the remaining part of the theorem let $\varphi$ be a rule that satisfies the properties listed in the theorem.

Then, by Lemma 4.13, $\varphi$ satisfies peak-onliness. Hence, by Theorem 4.3, $\varphi=U^{r}$.

Another possibility to prove Theorem 4.5 is to adjust Thomson's proof for the allocation model to our reallocation model. The interested reader can find this version of the proof in Appendix B.

Thomson (1995d, Theorem 4) conjectures that the uniform rule $U^{r}$ is the only rule that satisfies Pareto optimality, weak no-envy, one-sided replacement-domination, and replication-invariance. The following example shows that this conjecture is not true and it is not possible to weaken no-envy in Theorem 4.5 to either weak no-envy or equal treatment of equals.

Example 4.3 The following rule $\varphi^{3}$ satisfies Pareto optimality, weak no-envy, equal treatment of equals, one-sided replacement-domination, and replication-invariance. If $N \in \mathcal{N}$, and $e=(R, \omega) \in \mathcal{E}^{N}$ such that $z(e)<0$ and for all $i \in D(e), \omega_{i}=0$, then

$$
\varphi^{3}(e)= \begin{cases}p\left(R_{i}\right) & \text { if } i \notin D(e) \\ U_{i}\left(R_{D(e)}, s(e)\right) & \text { if } i \in D(e) .\end{cases}
$$

For all remaining $N \in \mathcal{N}$ and $e=(R, \omega) \in \mathcal{E}^{N}, \varphi^{3}(e)=U^{r}(e)$. 


\subsubsection{One-sided endowment-monotonicity}

Finally, we consider a change of one agent's individual endowment, keeping the individual endowments of the remaining agents and the set of agents fixed. Endowmentmonotonicity requires that either all agents (weakly) lose or all (weakly) gain.

Endowment-monotonicity: For all $N \in \mathcal{N}$, all $e=(R, \omega) \in \mathcal{E}^{N}$, all $\bar{e}=(R, \bar{\omega}) \in$ $\mathcal{E}^{N}$, and all $j \in N$, if $\bar{\omega}$ is a $j$-deviation from $\omega$, then either [for all $i \in N, \varphi_{i}(e) R_{i} \varphi_{i}(\bar{e})$ ] or [for all $\left.i \in N, \varphi_{i}(\bar{e}) R_{i} \varphi_{i}(e)\right]$.

The following example shows that similar to the allocation model, the properties Pareto optimality, weak no-envy, and endowment-monotonicity are generally incompatible.

Example 4.4 Let the rule $\varphi$ satisfy Pareto optimality, weak no-envy, and endowmentmonotonicity. Let $N=\{1,2\}$ and $e=(R, \omega) \in \mathcal{E}^{N}$ such that $p(R)=(0,4), 5 P_{2} 3$, and $\omega=(1,2)$. Since $z(e)>0$, by same-sidedness it follows that $\varphi(e)=(0,3)$. Next, consider $\bar{e}=(R, \bar{\omega}) \in \mathcal{E}^{N}$ such that $\bar{\omega}=(1,5)$. Since $z(e)<0$, by same-sidedness, it follows that for $i=1,2, \Delta \varphi_{i}(e) \geq-1$. Since $s_{1}(\bar{e})=s_{2}(\bar{e})$, by weak no-envy it follows that $\varphi(\bar{e})=\bar{\omega}$. Since $\bar{\omega}$ is a 2-deviation from $\omega$, in contradiction to endowmentmonotonicity, $\varphi_{1}(e) P_{1} \varphi_{1}(\bar{e})$ and $\varphi_{2}(\bar{e}) P_{2} \varphi_{2}(e)$.

However, the uniform reallocation rule satisfies Pareto optimality, weak no-envy, and the one-sided version of endowment-monotonicity, i.e., if the change of one agent's individual endowment is one-sided, then either all agents (weakly) lose or all (weakly) gain (see also Section 2.2 , page 24).

One-sided endowment-monotonicity: For all $N \in \mathcal{N}$, all $e=(R, \omega) \in \mathcal{E}^{N}$, all $\ddot{e}=(R, \vec{\omega}) \in \mathcal{E}^{N}$, and all $j \in N$, if $\bar{\omega}$ is a $j$-deviation from $\omega$ and $\bar{e}$ is a one-sided change of $e$, then either [for all $i \in N, \varphi_{i}(e) R_{i} \varphi_{i}(\bar{e})$ ] or [for all $\left.i \in N, \varphi_{i}(\bar{e}) R_{i} \varphi_{i}(e)\right]$.

The proportional rule $\mathrm{Pr}^{r}$ and the uniform rule $U^{r}$ satisfy one-sided endowmentmonotonicity.

Lemma 4.14 The uniform rule $U^{r}$ satisfies one-sided endowment-monotonicity.

Proof: Let $N \in \mathcal{N}, e=(R, \omega) \in \mathcal{E}^{N}$, and $\bar{e}=(\tilde{R}, \omega) \in \mathcal{E}^{N}$ be such that $\bar{\omega}$ is a $j$-deviation from $\omega$ and $\bar{e}$ is a one-sided change of $e$. In order to prove one-sided endowment-monotonicity, we have to show that either (i) [for all $i \in N, U_{i}^{r}(e) R_{i} U_{i}^{r}(\bar{e})$ ] or (ii) [for all $\left.i \in N, U_{i}^{r}(\bar{e}) R_{i} U_{i}^{r}(e)\right]$.

Assume, without loss of generality, that $z(e) \leq 0, z(\bar{e}) \leq 0$, and $\bar{\omega}_{j}>\omega_{j}$. Hence, for all $i \in N, U_{i}^{r}(e)=\max \left\{p\left(R_{i}\right), \omega_{i}+\lambda\right\}$, where $\lambda$ solves $\sum_{N} U_{i}^{r}(e)=\sum_{N} \omega_{i}$ and $U_{i}^{r}(\bar{e})=\max \left\{p\left(R_{i}\right), \bar{\omega}_{i}+\bar{\lambda}\right\}$, where $\bar{\lambda}$ solves $\sum_{N} U_{i}^{r}(\bar{e})=\sum_{N} \bar{\omega}_{i}$. By the definition of $U^{r}$ it follows that $\bar{\lambda} \geq \lambda$. Hence, for all $i \in N, p\left(R_{i}\right) \leq U_{i}^{r}(e) \leq U_{i}^{r}(\bar{e})$. This implies (i). 
A stronger notion of one-sided endowment-monotonicity is the following strong onesided endowment-monotonicity. If in case of excess demand an agent's individual endowments decreases, then no agent is made better off in the reallocation that follows this one-sided change. Similarly, if in case of excess supply an agent's individual endowments increases, then no agent is made better off in the reallocation that follows this one-sided change.

Strong one-sided endowment-monotonicity: For all $N \in \mathcal{N}$, all $e=(R, \omega) \in \mathcal{E}^{N}$, all $\bar{e}=(R, \bar{\omega}) \in \mathcal{E}^{N}$, and all $j \in N$, if $\bar{\omega}$ is a $j$-deviation from $\omega$ and $\bar{\omega}_{j}<\omega_{j}$, then [if $z(e) \geq 0$, then for all $i \in N, \varphi_{i}(e) R_{i} \varphi_{i}(\bar{e})$ ] and [if $z(\bar{e}) \leq 0$, then for all $i \in N$, $\left.\varphi_{i}(\vec{e}) R_{i} \varphi_{i}(e)\right]$.

Next, we prove that Pareto optimality and one-sided endowment-monotonicity together imply strong one-sided endowment-monotonicity.

Lemma 4.15 Let the rule $\varphi$ satisfy Pareto optimality and one-sided endowment-monotonicity. Then $\varphi$ satisfies strong one-sided endowment-monotonicity.

Proof. Let $\varphi$ be a rule that satisfies Pareto optimality and one-sided endowmentmonotonicity. Let $N \in \mathcal{N}, e=(R, \omega) \in \mathcal{E}^{N}, \bar{e}=(R, \bar{\omega}) \in \mathcal{E}^{N}$, and $j \in N$ be such that $\bar{\omega}$ is a $j$-deviation from $\omega$ and $\bar{\omega}_{j}<\omega_{j}$. Hence, $\sum_{i \in N} \bar{\omega}_{i}<\sum_{i \in N} \omega_{i}$. Suppose, without loss of generality, that $z(\bar{e}) \leq 0$. Hence, $z(e)<0$. Then, by one-sided endowmentmonotonicity, either (i) [for all $i \in N, \varphi_{i}(e) R_{i} \varphi_{i}(\bar{e})$ ] or (ii) [for all $i \in N, \varphi_{i}(\bar{e}) R_{i} \varphi_{i}(e)$ ]. In order to prove strong one-sided endowment-monotonicity we have to show that (ii) holds. Suppose, by contradiction, that (i) holds. Then, by same-sideness, for all $i \in N$, $p\left(R_{i}\right) \leq \varphi_{i}(e) \leq \varphi_{i}(\bar{e})$. So, by feasibility, $\sum_{i \in N} \omega_{i}=\sum_{i \in N} \varphi_{i}(e) \leq \sum_{i \in N} \varphi_{i}(e)=$ $\sum_{i \in N} \bar{\omega}_{i}$. This is in contradiction to $\sum_{i \in N} \bar{\omega}_{i}<\sum_{i \in N} \omega_{i}$.

The (one-sided) endowment-monotonicity properties introduced above can be seen as extensions of (one-sided) resource-monotonicity introduced for allocation rules (see Chapter 2). Other variations of endowment monotonicity for the reallocation setting are discussed in Thomson (1995d). In Klaus, Peters, and Storcken (1997b) and Klaus (1997b) a slightly stronger notion of (strong) one-sided endowment-monotonicity is applied.

Next, we show that the uniform rule $U^{r}$ is the only rule that satisfies Pareto op timality, weak no-envy; and one-sided endowment-monotonicity. This characterization can be seen as an extension of Thomson's characterization of the uniform allocation rule by Pareto optimality, no-envy, and one-sided resource-monotonicity for the domain of economies with bounded preferences (see Theorem 2.5). However, the proof of this characterization in the reallocation setting is based on a different proof technique and holds for the whole domain of single-peaked preferences. 
The stages of the proof of this characterization are as follows. First we show that Pareto optimality and one-sided endowment-monotonicity together imply "coordinatewise endowment continuity (see Lemma 416). Next we show that rules that satisfy Pareto optimality, weak no-envy, and one-sided endowment-monotonicity have the so-called dummy property: non-traders receive zero allotment changes (see Lemma 4.17). Then, we show that rules that satisfy Pareto optimality, one-sided endowmentmonotonicity, and the dummy property satisfy boundedness by endowments and peaks: each agent's allotment is between his individual endowment and his peak (see Lemma 4.18). Applying these results, the characterization we mentioned above follows easily.

Let $N \in \mathcal{N}, e=\left(R_{,} \omega\right) \in \mathcal{E}^{N}, j \in N$, and $\alpha \in \mathbb{R}_{+}$. Then, we define the $j$-deviation $\omega(\alpha, j) \in \mathbb{R}_{+\infty}^{N}$ from $\omega$ by $\omega(\alpha, j)_{j}=\alpha$ and $\omega(\alpha, j)_{i}=\omega_{i}$ for all $i \in N \backslash\{j\}$. Agent $j$ 's individual endowment after the unilateral change is $\alpha$. Now, continuity of a rule with respect to unilateral endowment changes can be formalized as follows.

Coordinatewise endowment continuity: For all $N \in \mathcal{N}$, all $e=\left(R_{n}, \omega\right) \in \mathcal{E}^{N}$, all $j \in N$, and all sequences $\left\{\alpha^{k}\right\}_{k \in l}$, if $\lim _{k \rightarrow \infty} \alpha^{k}=\omega_{j}$, then

$\lim _{k \rightarrow \infty} \varphi\left(R, \omega\left(\alpha^{k}, j\right)\right)=\varphi(e)$.

The following lemma states that Pareto optimality and one-sided endowment-monotonicity together imply coordinatewise endowment continuity. A similar result for allocation problems can be found in Thomson (1994a) in the proof of Theorem 2.

Lemma 4.16 Let the rule $\varphi$ satisfy Pareto optimality and one-sided endowment-mo notonicity. Then $\varphi$ satisfies coordinatewise endowment continuity.

Let $x, y \in \mathbb{R}^{N}$. Then, $x \geq y$ means that for all $i \in N, x_{i} \geq y_{i}$.

Proof. Let $\varphi$ be a rule that satisfies Pareto optimality and one-sided endowmentmonotonicity. Then, by Lemma 4.15, $\varphi$ satisfies strong one-sided endowment-monotonicity.

Let $N \in \mathcal{N}_{,} e=(R, \omega) \in \mathcal{E}^{N}, j \in N$, and $\left\{\alpha^{k}\right\}_{k \in \mathbb{N}}$ be a sequence in $\mathbb{R}_{+}$such that $\lim _{k \rightarrow \infty} \alpha^{k}=\omega_{j}$. Let for all $k \in \mathbb{N}_{,} x^{k}=\varphi\left(R, \omega\left(\alpha^{k}, j\right)\right)$. In order to prove coordinatewise continuity we have to show that $\lim _{k \rightarrow+\infty} x^{k}=\varphi(e)$. Suppose, without loss of generality, that $\alpha^{1}>\alpha^{2}>\alpha^{3}>\ldots>\omega_{j}$. We distinguish two cases.

Case 1: $z(e) \leq 0$. Then, for all $k \in \mathbb{N}, z\left(R, \omega\left(\alpha^{k}, j\right)\right)<0$. Hence, by same-sidedness, it follows for all $k \in \mathbb{N}, \varphi(e) \geq p(R)$ and $x^{k} \geq p(R)$. Now; by strong one-sided endowment-monotonicity, it follows that for all $k \in \mathbb{N}, x^{k} \geq \varphi(e)$ and the sequence $\left\{x^{k}\right\}_{k \in \mathbb{N}}$ is non-increasing. By feasibility, it follows that for all $k \in \mathbb{N}, \sum_{i \in N} x_{i}^{k}=$ $\sum_{i \in N} \omega\left(\alpha^{k}, j\right)_{i}$ and $\sum_{i \in N} \varphi_{i}(e)=\sum_{i \in N} \omega_{i}$. Because $\omega\left(\alpha_{i}^{k} j\right)_{j}=\alpha^{k}$ converges to $\omega_{i}, x^{t}$ converges to $\varphi(e)$.

Case 2: $z(e)>0$. Then, there exists $k_{0} \in \mathbb{N}$ such that $z\left(R, \omega\left(\alpha^{k 0}, j\right)\right)>0$ for all $k \geq k_{0}$. Without loss of generality, let $k_{0}=1$. The proof proceeds similar to Case 1 .

Next, we show that rules that satisfy Pareto optimality, no-envy, and one-sided endowment-monotonicity have the so-called dummy property: non-traders receive zero 
allotment changes.

Dummy property: For all $N \in \mathcal{N}$, all $e=(R, \omega) \in \mathcal{E}^{N}$, and all $i \in N$, if $p\left(R_{i}\right)=\omega_{i}$, then $\varphi_{i}(e)=\omega_{i}$.

Lemma 4.17 Let the rule $\varphi$ satisfy Pareto optimality, weak no-envy, and one-sided endowment-monotonicity. Then $\varphi$ satisfies the dummy property.

Proof. Let $\varphi$ be a rule that satisfies Pareto optimality, wreak no-envy, and one-sided endowment-monotonicity. Then, by Lemmas 4.15 and $4.16, \varphi$ satisfies strong one-sided endowment-monotonicity and coordinatewise endowment continuity.

Let $N \in \mathcal{N}, e=(R, \omega) \in \mathcal{E}^{N}$, and $j \in N$ be such that $p\left(R_{j}\right)=\omega_{j}$. In order to prove the dummy property we have to show that $\varphi_{j}(e)=\omega_{j}$. Suppose, by contradiction, that $\varphi_{j}(e) \neq \omega_{j}$. By Pareto optimality, this implies that either $z(e)>0$ or $z(e)<0$.

Case 1: $z(e)>0$. Then, by same-sidedness, $\varphi_{j}(e)<p\left(R_{j}\right)=\omega_{j}$. Furthermore, by strong one-sided endowment-monotonicity, we assume without loss of generality (lower the individual endowments if necessary) that all agents, except agent $j$, have either maximal demand $d_{\max }=\left\{p\left(R_{i}\right)-\omega_{i} \| i \in N\right\}$ or zero as individual endowment, i.e., for all $i \in N \backslash\{j\}, \omega_{i}=\max \left\{p\left(R_{i}\right)-d_{\max }, 0\right\}{ }^{18}$ Since $z(e)>0$, it follows that $d_{\max }>0$. Let $\alpha:=\omega_{j}-\varphi_{j}(e)<0$. For $0 \leq \varepsilon \leq \alpha$ we consider the endowment vector $\omega\left(\omega_{j}-\varepsilon, j\right)=: \omega^{\varepsilon}$, i.e., for all $i \in N \backslash\{j\}, \omega_{i}^{\varepsilon}=\omega_{i}$ and $\omega_{j}^{\varepsilon}=\omega_{j}-\varepsilon$. Hence, for $0 \leq \varepsilon \leq \alpha, \omega_{j} \geq \omega_{j}^{e} \geq \varphi_{j}(e)$. Let for all $i \in N, \alpha_{i}^{\varepsilon}:=\Delta \varphi_{i}\left(R, \omega^{\varepsilon}\right)$. By weak no-envy between $j$ and agents $i \in N \backslash\{j\}$ and strong one-sided endowment-monotonicity it follows that for all $i \in N \backslash\{j\}$ and $0 \leq \varepsilon<\alpha$, either $\omega_{j}^{\varepsilon}+\alpha_{i}^{\varepsilon}>p\left(R_{j}\right)$ or $\omega_{j}^{\varepsilon}+\alpha_{i}^{\varepsilon} \leq \omega_{j}^{\varepsilon}+\alpha_{j}^{\varepsilon}=\varphi_{j}\left(R, \omega^{\varepsilon}\right) \leq \varphi_{j}(e)<\omega_{j}^{\varepsilon}$. Because $p\left(R_{j}\right)-\omega_{j}^{\varepsilon}=\varepsilon$ this implies for all $i \in N \backslash\{j\}$ and all $0 \leq \varepsilon<\alpha$, either (i) $\alpha_{i}^{\varepsilon}>\varepsilon$ or (ii) $\alpha_{i}^{\varepsilon} \leq \alpha_{j}^{\varepsilon} \leq \varphi_{j}(e)-\omega_{j}^{\varepsilon}<0$.

Next, suppose that for some $k \in N \backslash\{j\}$ and some $\varepsilon, 0 \leq \varepsilon<a, \alpha_{k}^{E}<0$. Then, by feasibility, $\omega_{i}^{\varepsilon}=\omega_{i}>0$. Note, that by same-sidedness, for all $i \in N, \alpha_{i}^{\varepsilon} \leq d_{\max }=$ $d_{k}\left(R, \omega^{\varepsilon}\right)$. Then, by weak no-envy, it follows that for all $i \in N \backslash\{k\}, \alpha_{i}^{e} \leq \alpha_{k}^{\varepsilon}$. Thus, for all $i \in N, \alpha_{i}^{\varepsilon}<0$. This contradicts feasibility. Hence, for all $i \in N \backslash\{j\}$ and all $\varepsilon$, $0 \leq \varepsilon<\alpha, \alpha_{k}^{\varepsilon} \geq 0$. This implies for all $i \in N \backslash\{j\}$ and all $0 \leq \varepsilon<\alpha, \alpha_{i}^{\varepsilon}>\varepsilon$.

By strong one-sided endowment-monotonicity it follows that for all $i \in N$ and all $0 \leq \tau \leq \varepsilon \leq \alpha, \alpha_{i}^{\tau} \geq \alpha_{i}^{\varepsilon}$. Particularly it follows that $\alpha_{j}^{0}=-\alpha \geq \alpha_{j}^{\alpha}$. By coordinatewise endowment continuity, for all $i \in N \backslash\{j\}, \alpha_{i}^{\alpha} \geq \alpha$. This is only possible if $N=\{i, j\}$, $\alpha_{i}^{\alpha}=\alpha$ and $\alpha_{j}^{\alpha}=-\alpha$. Hence, $j$ envies $i$.

Case 2: $z(e)<0$. Similar to Case 1 .

In the next lemma, we show that Pareto optimality, one-sided endowment-monotonicity, and the dummy property imply boundedness by endowments and peaks: each agent's allotment is between his individual endowment and his peak.

\footnotetext{
${ }^{18}$ For problems in excess supply, assume that all suppliers have equal maximal supply.
} 
Boundedness by endowments and peaks: For all $N \in \mathcal{N}$, all $e=(R, w) \in \mathcal{E}^{N}$, and all $i \in N$, either $w_{i} \leq \varphi_{i}(e) \leq p\left(R_{i}\right)$ or $p\left(R_{i}\right) \leq \varphi_{i}(e) \leq \omega_{i}$

Boundedness by endowments and peaks implies the dummy property. It is easy to check that the uniform rule $U^{r}$ and the proportional rule $P_{r^{r}}$ satisfy boundedness by endowments and peaks. Furthermore, boundedness by endowments and peaks implies individual rationality: after the reallocation no agent is worse of than at his individual endowment (see also Section 2.2 , page 27 ).

Individual rationality: For all $N \in \mathcal{N}$, all $e=(R, \omega) \in \mathcal{E}^{N}$, and all $i \in N$, $\varphi_{i}(e) R_{j} \omega_{i}$.

The following lemma shows that under the assumptions of Pareto optimality and one-sided endowment-monotonicity the dummy property is equivalent to boundedness by endowments and peaks.

Lemma 4.18 Let the rule $\varphi$ satisfy Pareto optimality, the dummy property, and one-sided endowment-monotonicity. Then $\varphi$ satisfies boundedness by endowments and peaks.

Proof. Let $\varphi$ be a rulle that satisfies Pareto optimality, the dummy property, and one-sided endowment-monotonicity. Then, by Lemma $4.15, \varphi$ satisfies strong one-sided endowment-monotonicity.

Let $N \in \mathbb{N}$ and $e=(R, \omega) \in \mathcal{E}^{N}$.

Case 1: $z(e) \geq 0$. By same-sidedness, for all $i \in N, \varphi_{i}(e) \leq p\left(R_{i}\right)$. In order to prove boundedness by endowments and peaks we have to show that $\omega_{i} \leq \varphi_{i}(e) \leq p\left(R_{i}\right)$. Suppose that there exists a $j \in N$ such that $\varphi_{j}(e)<p\left(R_{j}\right)$. Now, it is sufficient to prove that $\omega_{j} \leq \varphi_{j}(e)$. Consider $\omega \in \mathbb{R}_{+}^{N}$ such that for all $i \in D(e), \bar{\omega}_{i}=\omega_{i}$ and for all $i \notin D(e), \omega_{i}=p\left(R_{i}\right)$. Let $\bar{e}=(R, \bar{\omega}) \in \mathcal{E}^{N}$. By strong one-sided endowmentmonotonicity it follows that for all $i \in N, \varphi_{i}(\ddot{e}) \leq \varphi_{i}(e)$. By the dummy property, for all $i \notin D(\bar{e}), \varphi_{i}(\bar{e})=p\left(R_{i}\right)$. Therefore $j \in D(e)=D(\bar{e})$. Now, by strang one-sided endowment-monotonicity it is sufficient to prove that $\omega_{j}=\bar{\omega}_{j} \leq \varphi_{j}(\bar{e})$. Suppose to the contrary that $\varphi_{j}\left(\bar{e}^{n}\right)<\bar{\omega}_{j}$.

Consider $\tilde{\omega}=\bar{\omega}\left(p\left(R_{j}\right), j\right) \in \mathbb{R}_{+}^{N}, i . e$. for all $i \in N \backslash\{j\}, \tilde{\omega}_{i}=\omega_{i}$ and $\tilde{\omega}_{j}=p\left(R_{j}\right)$. Let $\tilde{e}=(R, \tilde{\omega}) \in \mathcal{E}^{N}$. Because $\varphi_{j}(\bar{e})<\bar{e}_{j}$, it follows by same-sidedness and feasibility that $D(\bar{e}) \neq\{j\}$. Hence $z(\tilde{e})>0$. Therefore, by strong one-sided endowment-monotonicity, it follows that for all $i \in N, \varphi_{i}(\bar{e}) \leq \varphi_{i}(\tilde{e})$. By feasibility this yields $\varphi_{j}(\tilde{e}) \leq \varphi_{j}(\bar{e})+$ $p\left(R_{j}\right)-\tilde{\omega}_{j}<p\left(R_{j}\right)=\tilde{\omega}_{j}$. This, however, contradicts the dummy property. So, $\varphi_{j}(\bar{e}) \geq$ $\bar{\omega}_{j}$

Case 2: $z(e)<0$. Similar to Case 1 .

Finally, we prove the characterization of the uniform rule $U^{r}$ by Pareto optimality, weak no-envy, and one-sided endowment-monotonicity. As already mentioned above, 
this result can be interpreted as an extension of 'Thomson's Theorem 2.5 to the reallocation model.

Theorem 4.6 ${ }^{19}$ The uniform rule $U^{r}$ is the only rule that satisfies Pareto optimality, weak no-envy, and one-sided endowment-monotonicity.

Proof. By same-sidedness and Lemmas 4.6 and 4.14 it follows that the uniform rule $U^{r}$ satisfies Pareto optimality, weak no-envy, and one-sided endowment-monotonicity.

To prove the remaining part of the theorem let $\varphi$ be a rule that satisfies the properties listed in the theorem. Then, by the Lemmas $4.15,4.16,4.17$, and $4.18, \varphi$ satisfies strong one-sided endowment-monotonicity, coordinatewise endowment continuity, and boundedness by endowments and peaks.

Let $N \in \mathcal{N}, e=(R, \omega) \in \mathcal{E}^{N}$, and suppose, by contradiction, that $\varphi(e) \neq \mathcal{U}^{r}(e)$. Since $\varphi$ is Pareto optimal it follows that either $z(e)>0$ or $z(e)<0$.

Case 1: $\quad z(e)>0$. By same-sidedness and boundedness by endowments and peaks it follows that for all $i \notin D(e) ; \varphi_{i}(e)=p\left(R_{i}\right)=U_{i}^{r}(e)$. Then, by Lemma 4.1, there exist agents $j, k \in D(e)$ such that $\varphi_{j}(e)<p\left(R_{j}\right)$ and $\Delta \varphi_{j}(e)<\Delta \varphi_{k}(e)$. By no-enry, $\omega_{j}+\Delta \varphi_{k}(e)>p\left(R_{j}\right)$. Let $l \in S(e)$ and consider $\omega^{\alpha}=\omega(\alpha, l) \in \mathbb{R}_{+}^{N}$ such that $p\left(R_{l}\right) \leq$ $\alpha \leq \omega_{i}$ and $\alpha \longrightarrow p\left(R_{i}\right)$. Let $e^{\alpha}=\left(R, \omega^{\alpha}\right) \in \mathcal{E}^{N}$. Then, by coordinatewise endowment continuity, strong one-sided endowment-monotonicity, and no-envy, it follows that for $\alpha^{\prime}=p\left(R_{l}\right), \omega_{j}^{\alpha^{\prime}}+\Delta \varphi_{k}\left(e^{\alpha^{\prime}}\right)>p\left(R_{j}\right)$. Note that $l$ is a non-trader at problem $e^{\alpha^{\prime}}$. Hence, the set of suppliers has been decreased by one. Repeating this process yields a problem, say $\tilde{e}=(R, \tilde{\omega})$, such that for all $i \in D(e), \tilde{\omega}_{i}=\omega_{i}$, for all $i \notin D(e), \tilde{\omega}_{i}=p\left(R_{i}\right)$, and $\tilde{\omega}_{j}+\Delta \varphi_{k}(\tilde{e})>p\left(R_{j}\right)$. In particular, we have $\Delta \varphi_{k}(\tilde{e})>0$. But then either feasibility or boundedness by endowments and peaks is violated.

Case 2: $\quad z(e)<0$. Similar to Case 1 .

In the remainder of this section, we derive a characterization of the uniform rule $U^{*}$ in terms of individual rationality, strong one-sided endowment-monotonicity, and weak no-envy (see Corollary 4.9). First, we show that individual rationality and strong one-sided endowment-monotonicity imply Pareto optimality.

Lemma 4.19 Let the rule $\varphi$ satisfy individual rationality and strong one-sided endowment-monotonicity. Then $\varphi$ satisfies Pareto optimality.

Proof. Let $\varphi$ be a rule that satisfies individual rationality and strong one-sided endowment-monotonicity. Let $N \in \mathcal{N}, e=(R, \omega) \in \mathcal{E}^{N}$, and suppose, by contradiction, that $\varphi(e)$ is not same-sided.

\footnotetext{
19.Theorem 4.6 is a strengthening of a result of Klaus, Peters, and Storcken $(1997 \mathrm{~b}$, Theorerr. 4.4): the uniform rule $U^{r}$ is the only rule that satisfies Pareto optimality, no-envy, and a stronger version of one-sided endowment-monotonicity. In Klaus, Peters, and Storcken (1997b), we did not succoed to prove the independence of the properties in the characterization mentioned above. The properties in Theorem 4.6 are independent (see Section 4.6 ).
} 
Case 1: $z(e) \geq 0$. The proof proceeds as follows. In (1), we show that for $e$ the rule $\varphi$ assigns to all suppliers and all non-traders their peaks. ${ }^{20}$ In (ii), we define a sequence of economies $\left\{e^{q}\right\}$ gev such that for any $q \in \mathbb{N}$ the corresponding allotment $\varphi\left(e^{q}\right)$ is not same-sided. Finally, in (iii), for $q$ large enough we derive a contradiction to feasibility. (i) Suppose, by contradiction, that for some agent $j \notin D(e), \varphi_{j}(e) \neq p\left(R_{j}\right)$. By individual rationality, $p\left(R_{j}\right) P_{j} \varphi_{j}(e) R_{j} \omega_{j}$

Now, let $\bar{\omega}=\omega\left(p\left(R_{j}\right), j\right) \in \mathbb{R}_{w}^{N}$ and $\bar{e}=(R, \bar{\omega}) \in \mathcal{E}^{N}$. Then, in $\bar{e}$, agent $j$ is a non-trader. Hence, by individual rationality in $\bar{e}, \varphi_{j}(\bar{e})=p\left(R_{j}\right)$. Note that $\bar{\omega}$ is a $j$-deviation from $\omega, \bar{\omega}_{j}<\omega_{j}$, and $z(e) \geq 0$. Then, by strong one-sided endowmentmonotonicity applied to $\bar{e}$ and $e_{i} p\left(R_{j}\right) P_{j} \varphi_{j}(e) R_{j} \varphi_{j}(\bar{e})$. Thus, $\varphi_{j}(\bar{e}) \neq p\left(R_{j}\right)$. This is a contradiction to individual rationality. Hence,

$$
\varphi_{j}(e)=p\left(R_{j}\right) \text { for all } j \notin D(e) \text {. }
$$

(ii) Since $\varphi(e)$ is not same-sided, by (i), there exists a demander $k \in D(e)$ such that $\varphi_{k}(e)>p\left(R_{k}\right)>\omega_{k}$. By individual rationality in $e$, there exists a point $a^{1} \in \mathbb{R}_{+}$such that

Let $d^{1}:=p\left(R_{k}\right)-a^{1}$.

$$
p\left(R_{k}\right)>a^{1} \geq \omega_{k} \text { and } \varphi_{k}(e) I_{k} a^{1}
$$

In $e$, by feasibility and individual rationality for all demanders, there exists at least one supplier l. Now, let $\omega^{1} \in \mathbb{R}_{+}^{N}$ be such that $\omega_{i}^{1}=\omega_{i}$ for $i \in N \backslash\{l\}$ and $\omega_{l}^{1}=\omega_{l}-\min \left\{\omega_{l}-p\left(R_{l}\right), d^{\mathbb{L}}\right\}$. Let $e^{1}=\left(R, \omega^{1}\right) \in \mathcal{E}^{N}$. Note that $D(e)=D\left(e^{1}\right)$. Also, note that $\omega^{1}$ is a $l$-deviation from $\omega_{1} \omega_{l}^{1}<\omega_{l}$, and $z\left(e^{1}\right) \geq 0$. Then, by strong one-sided endowment monotonicity applied to $e^{1}$ and $e$,

$$
\varphi_{i}(e) R_{i} \varphi_{i}\left(e^{1}\right) \text { for all } i \in N \text {. }
$$

Hence, by (4.8) and (4.9) it follows that for agent $k$, either

$$
\varphi_{k}\left(e^{1}\right) \geq \varphi_{k}(e)>p\left(R_{k}\right)
$$

or

If $(4.10)$, then

$$
p\left(R_{k}\right)>a^{1} \geq \varphi_{k}\left(e^{1}\right) \geq \omega_{k}
$$

$$
\varphi_{k}\left(e^{1}\right)>p\left(R_{k}\right)
$$

If (4.11), then the allotment of agent $k$ moves to the other side of his peak so that by construction $\varphi_{k}(e)>\varphi_{k}\left(e^{l}\right)+\min \left\{\omega_{l}-p\left(R_{l}\right), d^{\mathbb{1}}\right\}$.

By (4.7), applied to both $e$ and $e^{1}$, and by feasibility,

$$
\begin{aligned}
\sum_{i \in D(e)} \varphi_{i}(e) & =\sum_{i \in D(e)} \varphi_{i}\left(e^{1}\right)+\min \left\{\omega_{l}-p\left(R_{l}\right), d^{1}\right\} \\
& =\sum_{\substack{i \in D(e) \\
i \neq k}} \varphi_{i}\left(e^{1}\right)+\varphi_{k}\left(e^{1}\right)+\min \left\{\omega_{l}-p\left(R_{l}\right), d^{l}\right\} .
\end{aligned}
$$

\footnotetext{
${ }^{20}$ This statement holds for arbitrarily chosem problems with excess demand or which are balanced. Similarly, for problems with excess supply, all demanders and non-traders receiwe their peaks. See also Thomson (1995d), Lemma 4.
} 
Since $\varphi_{k}(e)>\varphi_{k}\left(e^{1}\right)+\min \left\{\omega_{l}-p\left(R_{l}\right), d^{1}\right\}$, in $e^{1}$ there exists a demander $m$ such that $\varphi_{m}\left(e^{1}\right)>\varphi_{m}(e)$. Hence, by $(4.9)$,

$$
\varphi_{m}\left(e^{1}\right)>p\left(R_{m}\right) .
$$

Since either (4.12) or (4.13) hold, it follows that $\varphi\left(e^{1}\right)$ is not Pareto optimal. Now, we repeat the argument, proceeding from $e^{i}$ instead of $e$.

At each step $n \in \mathbb{N}$, the allotment $\varphi\left(e^{n}\right)$ is not same-sided and there exists $i \in D\left(e^{n}\right)$ such that $\varphi_{i}\left(e^{n}\right)>p\left(R_{i}\right)>a^{n} \geq \omega_{i}^{n}$ and $\varphi_{i}\left(e^{n}\right) I_{i} a^{n}$. Hence,

$$
d^{n+1}=p\left(R_{i}\right)-a^{n}>0 .
$$

In step $n+1$ we lower the endowment of some $j \in S\left(e^{n}\right)$ by $\min \left\{\omega_{j}^{n}-p\left(R_{j}\right), d^{n+1}\right\}$. This procedure yields

$$
\begin{array}{ll}
D(e)=D\left(e^{q}\right) & \text { for all } q \in \mathbb{N} \text { and } \\
\left\{\varphi\left(e^{q}\right)\right\}_{q \in \mathbb{N}}, & \text { an infinite sequence of reallocations } \\
& \text { which are not Pareto optimal. }
\end{array}
$$

(iii) Since the set of demanders $D:=D(e)=D\left(e^{q}\right)$ is finite, by (4.15) it follows that at least one demander must be several times on the "wrong side" of his peak. Consider such an $i \in D$. Let $s>n$ be such that $\varphi_{i}\left(e^{s}\right)>p\left(R_{i}\right)$ and $\varphi_{i}\left(e^{n}\right)>$ $p\left(R_{i}\right)$. Note that $\omega^{s} \leq \omega^{n}$ and $z\left(e^{n}\right) \geq 0$. Then, by strong one-sided endowment monotonicity applied to $\left[e^{n}\right.$ and $\left.e^{n+1}\right],\left[e^{n+1}\right.$ and $\left.e^{n+2}\right], \ldots,\left[e^{s-1}\right.$ and. $\left.e^{s}\right]$ it follows that $\varphi_{i}\left(e^{n}\right) R_{i} \varphi_{i}\left(e^{n+1}\right) R_{i} \varphi_{i}\left(e^{n+2}\right) R_{i} \ldots R_{i} \varphi_{i}\left(e^{s}\right)$. Thus, $\varphi_{i}\left(e^{s}\right) \geq \varphi_{i}\left(e^{n}\right)$. Hence, $a^{s} \leq a^{n}$ and therefore

$$
d^{s+1} \geq d^{n+1} \text { for } s>n \text {. }
$$

Because the number of demanders is finite, (4.14) and (4.16) imply that the sequence $\left\{d^{q}\right\}_{q \in \mathbb{A}}$ is bounded from below by a term $d>0$ of the sequence. So, at each step, the endowment of a supplier is lowered by at least $d$ without this turning him into a demander. Repeating this procedure, all suppliers are made non-traders after a finite number $t, t \in \mathbb{N}$, of steps. For problem $e^{t}$, it follows that

$$
\varphi_{j}\left(e^{t}\right)=p\left(R_{j}\right)=\omega_{j}^{t} \text { for } j \notin D\left(e^{t}\right) .
$$

Then, by feasibility and individual rationality in $e^{t}$,

$$
\varphi_{i}\left(e^{t}\right)=\omega_{i} \text { for } i \in D\left(e^{t}\right) .
$$

Note that $\varphi\left(e^{t}\right)$ is a term of the sequence $\left\{\varphi\left(e^{g}\right)\right\}_{q \in \mathbb{N}}$ which is same-sided and therefore Pareto optimal. This contradicts (4.15).

Case 2: $z(e)<0$. Similar to Case 1 .

For the next theorem see also Thomson (1995d), Theorem 2. 
Theorem 4.7 On the domain of two-agent economies, $\bigcup_{\substack{N \in \mathcal{N} \\|N|=2}} \mathcal{E}^{N}$, the uniform rule $U^{*}$ is the only rule that satisfies individual rationality and strong one-sided endowment monotonicity.

Proof. The uniform rule $U^{r}$ satisfies individual rationality and strong one-sided endowment monotonicity. To prove the remaining part of the theorem let $\varphi$ be a rule that satisfies the properties listed in the theorem. Let $N \in \mathcal{N}, N=\{i, \hat{j}\}$, and $e=(R, \omega) \in \mathcal{E}^{N}$.

Case 1: $U^{r}(e)=\omega$. Then, by individual rationality, $\varphi(e)=\omega=U^{r}(e)$.

Case 2: $U^{r}(e) \neq \omega$. This can only occur if agents are of "opposite" types, i.e., $N$ consists of one demander and one supplier. Suppose that $z(e) \geq 0$ and, without loss of generality, let $i \in S(e)$. Then, similarly as in the proof of Lemma 4.18 , part (i), it follows that $\varphi_{i}(e)=p\left(R_{i}\right)=U_{i}^{r}(e)$. Thus, $\varphi_{j}(e)=\omega_{j}+\left(\omega_{i}-p\left(R_{i}\right)\right)=U_{j}^{r}(e)$. For $z(e)<0, \varphi(e)=U^{r}(e)$ follows similarly.

Remark 4.3 Theorem 4.7 only applies to the two-agent case. As already established in the proof, a "real reallocation" only takes place when agents of opposite types exist. In this case one of the agents receives his peak; suppose, without loss of generality, that this is the supplier. Then, his total supply has to be allocated to the demander. For more than two agents, again the agents of one group (e.g., the suppliers and the non-traders) receive their peaks and the total supply of this group is distributed among the demanders. This can be done in different ways. All rules that are same-sided and distribute the total supply "monotonically" among the demanders are individually rational and strong one-sided endowment monotonic for problems with excess demand. A similar statement holds for excess supply. Examples of such rules are the uniform rule $U^{r}$, but also the proportional rule $P \tau^{r}$.

Corollary $4.9^{21}$ The uniform rule $U^{r}$ is the only rule that satisfies individual ratio nality, strong one-sided endowment monotonicity, and weak no-envy.

Proof. The uniform rule $U^{r}$ satisfies individual rationality, strong one-sided endowment monotonicity, and weak no-envy. To prove the remaining part of the corollary let $\varphi$ be a rule that satisfies the properties listed in the corollary. By Lemma $4.19, \varphi$ is Pareto optimal. Then, by Theorem 4.6, $\varphi=U^{r}$.

A summary of the characterizations of the uniform rule $U^{r}$ by Pareto optimality or individual rationality, one of the two no-envy properties, and one of the solidarity properties can be found in Table 4.2. For a comparison with corresponding characterizations of the uniform allocation rule $U$ we refer to Table 2.2 .

\footnotetext{
${ }^{21}$ We did not succed in proving the independence of this characterization. To be more precisely, it is an open question whether individual rationality is independent of weak no-envy and strong one-sided endowment-monotonicity.
} 


\begin{tabular}{|l||c|c|c|c|}
\hline & Th & Th & Th & Co \\
\hline \hline Pareto optimality & 4.4 & 4.5 & 4.6 & 4.9 \\
\hline no-envy & $\times$ & $\times$ & $\times$ & \\
\hline weak no-nvy & & $\times$ & & \\
\hline one-sided population-monotonicity & $\times$ & & & \\
\hline one-sided replacement-domination & & $\times$ & & \\
\hline one-sided endowment-monotonicity & & & $\times$ & \\
\hline strong one-sided endowment-monotonicity & & & & $\times$ \\
\hline replication-invariance & & $\times$ & & \\
\hline individual rationality & & & & $\times$ \\
\hline
\end{tabular}

Table 4.2: Characterizations of the uniform rule $U^{r}$ by Pareto optimality or individual rationality, no-envy properties, and solidarity properties.

\subsection{Consistency}

In this section we explore a notion of consistency. Consistency essentially describes the stability of a reallocation rule in situations where agents leave with their allotments: by leaving, these agents might create a positive or negative "leftover". Consider the reduced economy where this leftover is divided as equally as possible ${ }^{22}$ among the remaining agents. Then, consistency states that the agents' allotments for this reduced economy be equal to their allotments for the original economy (see also Section 2.2, page 26). For exchange economies, this notion of consistency can be found in Thomson (1992).

In the sequel, we consider the weaker notion of bilateral consistency which applies to situations where all but two agents leave with their allotments. Before we give the formal definition, we introduce some notation.

Let $N \in \mathcal{N}, e=(R, \omega) \in \mathcal{E}^{N}$, and $x=\varphi(e) \in \mathbb{R}_{+}^{N}$ be the reallocation assigned by the rule. Let $\{i, j\} \subseteq N, i \neq j$, and suppose that all agents in $N \backslash\{i, j\}$ leave with the allotments assigned to them at $x$. By doing so they create a leftover $\sum_{k \in N \backslash\{i, j\}}\left(\omega_{k}-x_{k}\right)$. Dividing this leftover as equally as possible among $i$ and $j$ yields the adjusted endowment vector $\omega(i, j) \in \mathbb{R}_{+}^{\{i, j\}}$ defined as follows.

If $\Delta \varphi_{i}(e) \leq \Delta \varphi_{j}(e)$, then

$$
\begin{aligned}
& \omega(i, j)_{j}=\max \left\{0, \omega_{j}+\frac{1}{2}\left(\Delta \varphi_{i}(e)+\Delta \varphi_{j}(e)\right)\right\} \geq 0 \text { and } \\
& \omega(i, j)_{i}=\omega_{i}+\left(\Delta \varphi_{i}(e)+\Delta \varphi_{j}(e)\right)-\left(\omega(i, j)_{j}-\omega_{j}\right) \geq 0 .
\end{aligned}
$$

If $\Delta \varphi_{i}(e) \geq \Delta \varphi_{j}(e)$, then

$$
\omega(i, j)_{i}=\max \left\{0, \omega_{i}+\frac{1}{2}\left(\Delta \varphi_{i}(e)+\Delta \varphi_{j}(e)\right)\right\} \geq 0 \text { and }
$$

\footnotetext{
${ }^{22}$ As equal as possible with respect to the domain restrictions.
} 


$$
\omega(i, j)_{j}=\omega_{j}+\left(\Delta \varphi_{i}(e)+\Delta \varphi_{j}(e)\right)-\left(\omega(i, j)_{i}-\omega_{i}\right) \geq 0
$$

So, endowment adjustments are as close as possible to the mean allotment changes of $i$ and $j .{ }^{23}$ We denote the reduced economy that is created in this way by $\left(R_{\{i, j\}}, \omega(i, j)\right) \in$ $\mathcal{E}^{\{t, j\}}$.

Bilateral consistency: For all $N \in \mathcal{N}$, all $e=(R, \omega) \in \mathcal{E}^{N}$, and all $i, j \in N, i \neq j$, $\varphi(e)_{\{i, j\}}=\varphi_{i}\left(R_{\{i, j\}}, \omega(i, j)\right)$.

Bilateral consistency for reallocation rules as introduced above can be seen as an extension of the bilateral consistency condition for allocation rules (see also Section 2.2, page 27). Note that by the definition of the reduced problem, an equity condition is embedded in our notion of bilateral consistency.

Next, we show that the uniform rule $U^{r}$ satisfies bilateral consistency. ${ }^{24}$

Lemma 4.20 The uniform rule $U^{r}$ satisfies bilateral consistency..

Proof. Let $N \in \mathcal{N}, e=(R, \omega) \in \mathcal{E}^{N}$, and $i, j \in N$ be such that $i \neq j$. Suppose, without loss of generality, that $\Delta U_{j}^{r}(e) \geq \Delta U_{i}^{r}(e)$. In order to prove bilateral consistency, we have to show that

$$
U^{r}(e)_{\{i, j\}}=U_{i}^{r}\left(R_{\{i, j\}}, \omega(i, j)\right)
$$

where $\omega(i, j)_{j}=\max \left\{0, \omega_{j}+\frac{1}{2}\left(\Delta U_{i}^{r}(e)+\Delta U_{j}^{r}(e)\right)\right\}$ and $\omega(i, j)_{i}=\omega_{i}+\left(\Delta U_{i}^{r}(e)+\right.$ $\left.\Delta U_{j}^{r}(e)\right)-\left(\omega(i, j)_{j}-\omega_{j}\right)$

Case 1: $z(e) \leq 0$.

Case 1.1: $\Delta U_{j}^{r}(e) \geq \Delta U_{i}^{r}(e) \geq 0$. Hence, $i, j \notin S(e)$ and $U^{r}(e)_{\{i, j\}}=p\left(R_{\{i, j\}}\right)$. Since $z\left(R_{\{i, j\}, \omega(i, j))}=0\right.$, by Pareto optimality of $U^{r}$, it follows that $U^{r}\left(R_{\{i, j\}, \omega}(i, j)\right)=$ $p\left(R_{\{i, j\}}\right)$. This implies (4.17).

Case 1.2: $\Delta U_{j}^{r}(e) \geq 0>\Delta U_{i}^{r}(e)$. Hence, $i \in S(e), j \notin S(e)$, and $U^{r}(e)_{\{i, j\}}=$ $\left(\omega_{i}-p\left(R_{j}\right)+\omega_{j}, p\left(R_{j}\right)\right)$. Since $z\left(R_{\{i, j\}}, \omega(i, j)\right) \leq 0, i \in S\left(R_{\{i, j\}}, \omega(i, j)\right)$, and $j \notin$ $S\left(R_{\left\{i_{i} j\right.}, \omega(i, j)\right)$ it follows that $U^{r}\left(R_{\{i, j\}}, \omega(i, j)\right)=\left(\omega_{i}-p\left(R_{j}\right)+\omega_{j}, p\left(R_{j}\right)\right)$. This implies (4.17).

Case 1.3: $\quad 0>\Delta U_{j}^{+}(e) \geq \Delta U_{i}^{r}(e)$. Hence, $i, j \in S(e)$ and $U^{r}(e)_{\{i, j\}}=\left(\max \left\{p\left(R_{i}\right), \omega_{i}+\right.\right.$ $\left.\lambda\}, \max \left\{p\left(R_{j}\right), \omega_{j}+\lambda\right\}\right)$ where $\lambda \in \mathbb{R}$ solves $\sum_{N} U_{k}^{r}(e)=\sum_{N} \omega_{k}$. We distinguish two subcases.

Case 1.3.1: $U_{j}^{r}(e)=p\left(R_{j}\right)$. Hence, $U^{r}(e)_{\{i, j\}}=\left(\max \left\{p\left(R_{i}\right), \omega_{i}+\lambda\right\}, p\left(R_{j}\right)\right)$ where $\lambda \in \mathbb{R}$ solves $\sum_{N} U_{k}^{r}(e)=\sum_{N} \omega_{k}$. Since $z\left(R_{\{i, j\}}, \omega(i, j)\right) \leq 0$ and $\Delta U_{j}^{r}(e) \geq \Delta U_{i}^{r}(e)$, it follows that $j \notin S\left(R_{\{i, j\}}, \omega(i, j)\right)$. Hence, $U_{j}^{r}\left(R_{\{i, j\}}, \omega(i, j)\right)=p\left(R_{j}\right)$ and, by feasibility, $U_{i}\left(R_{\{i, j\}}, \omega(i, j)\right)=\max \left\{p\left(R_{i}\right), \omega_{i}+\lambda\right\}$ where $\lambda \in \mathbb{R}$ solves $\sum_{N} U_{k}^{r}(e)=\sum_{N} \omega_{k}$. This implies (4.17).

\footnotetext{
${ }^{23}$ By just applying mean allotment changes, megative endowments, which are not admissible in this model, might occur.

${ }^{24}$ The uniform rule $U^{4}$ also satisfies consistency which we have not formalized here.
} 
Case 1.3.2: $\quad U_{j}^{r}(e)>p\left(R_{j}\right)$. Hence, $U^{r}(e)_{\{i, j\}}=\left(\omega_{i}+\lambda, \omega_{j}+\lambda\right)$. Since $z\left(R_{\left\{i_{j}\right\}}, \omega(\hat{j}, j)\right) \leq$ 0 and $\omega(i, j)=\left(\omega_{i}+\lambda, \omega_{j}+\lambda\right)>p\left(R_{\{i, j\}}\right)$, it follows that $U^{r}\left(R_{\{i, j\}, \omega(i, j))=\omega(i, j)}\right.$.
This implies $(4.17)$.

Case 2: $z(e)>0$. Similar to Case 1 .

The next result we prove is a characterization of the uniform rule $U^{r}$ by Pareto optimality, bilateral consistency, and boundedness by endowments and peaks.

Theorem 4.8 The uniform rule $U^{r}$ is the only rule that satisfies Pareto optimality, bilateral consistency, and boundedness by endowments and peaks.

Proof. By same-sidedness and Lemma 4.20 it follows that the uniform rule $U^{r}$ satisfies Pareto optimality and bilateral consistency. Furthermore, the uniform rule $U^{r}$ satisfies boundedness by endowments and peaks.

To prove the remaining part of the theorem let $\varphi$ be a rule that satisfies the properties listed in the theorem. Let $N \in \mathcal{N}$ and $e=(R, \omega) \in \mathcal{E}^{N}$. If $|N|=2$, then, by Pareto optimality and boundedness by endowments and peaks, it follows easily that $\varphi(e)=U^{r}(e)$. Now, let $|N| \geq 3$ and suppose, by contradiction, that $\varphi(e) \neq U^{r}(e)$. Since $\varphi$ is Pareto optimal it follows that either $z(e)>0$ or $z(e)<0$.

Case 1: $z(e)<0$. By boundedness by endowments and peaks and same-sidedness, it follows that for all $i \notin S(e), \varphi_{i}(e)=p\left(R_{i}\right)=U_{i}^{r}(e)$. Hence, by Lemma 4.1, there exist agents $j, k \in S(e)$ such that $\varphi_{j}(e)>p\left(R_{j}\right)$ and $\Delta \varphi_{j}(e)>\Delta \varphi_{k}(e)$. Because of boundedness by endowments and peaks, $\Delta \varphi_{j}(e) \leq 0$. Hence, $\omega(j, k)_{j}<\varphi_{j}(e) \leq$ $\omega_{j}$. By bilateral consistency it follows for the reduced economy $\left(R_{\{j, k\}}, \omega(j, k)\right)$ that $\varphi_{j}\left(R_{\{j, k\}}, \omega(j, k)\right)=\varphi_{j}(e)$. Hence, in contradiction to boundedness by endowments and peaks, $\varphi_{j}\left(R_{\{j, k\}}, \omega(j, k)\right)>p\left(R_{j}\right)$ and $\varphi_{j}\left(R_{\{j, k\}}, \omega(j, k)\right)>\omega(j, k)$.

Case 2: $z(e)>0$. Similar to Case 1 .

Since Pareto optimality, one-sided endowment-monotonicity and the durmmy property imply boundedness by endowments and peaks (see Lemma 4.18), Theorem 4.8 implies the following characterization of the uniform rule $U^{r}$.

Corollary 4.10 The uniform rule $U^{r}$ is the only rule that satisfies Pareto optimality, one-sided endowment-monotonicity, bilateral consistency, and the dummy property.

The following theorem shows that we can replace boundedness by endowments and peaks in Theorem 4.8 by the weaker property of individual rationality. This characterization can be seen as an extension of Dagan's characterization of the uniform allocation rule by Pareto optimality, bilateral consistency, and individual rationality from equal division (see Theorem 2.12).

Theorem 4.9 The uniform rule $U^{r}$ is the only rule that satisfies Pareto optimality, bilateral consistency, and individual rationality. 
Proof. The uniform rule $U^{r}$ satisfies Pareto optimality and bilateral consistency. Furthermore, the uniform rule $U^{r}$ satisfies individual rationality.

To prove the remaining part of the theorem let $\varphi$ be a rule that satisfies the properties listed in the theorem. By Theorem 4.8 it is sufficient to prove that $\varphi$ satisfies boundedness by endowments and peaks.

Let $N \in \mathcal{N}$ and $e=(R, \omega) \in \mathcal{E}^{N}$.

Case 1: $z(e) \leq 0$. By same-sidedness and individual rationality it follows that for all $i \notin D(e), w_{i} \geq \varphi_{i}(e) \geq p\left(R_{i}\right)$. For all $i \in D(e)$ it follows by same-sidedness that $\varphi_{i}(e) \geq p\left(R_{i}\right)$. Suppose, in contradiction to boundedness by endowments and peaks, that there exist $j \in D(e)$ such that $\varphi_{j}(e)>p\left(R_{j}\right)$. Hence, $|N| \geq 2$ and, by feasibility and individual rationality there exists $k \in S(e)$ such that $\Delta \varphi_{k}(e)<0$.

Case 1.1: $|N| \geq 3$. Hence, it follows that $\omega(j, k)_{j}<\varphi_{j}(e)$. By bilateral consistency it follows for the reduced economy $\left(R_{\{j, k\}}, \omega(j, k)\right)$ that $\varphi_{j}\left(R_{[j, k\}}, \omega(j, k)\right)=\varphi_{j}(e)$. So, by individual rationality,

$$
\omega(i, j)_{j} \leq p\left(R_{j}\right)<\varphi_{j}\left(R_{\{j, k\}}, \omega(j, k)\right) .
$$

Next, we enlarge the economy $\left(R_{\{j, k\}}, \omega(j, k)\right)$ by a non-trader $l \neq j, k$. Let $M=$ $\{j, k, l\}$ and consider the endowment vector $\bar{\omega} \in \mathbb{R}_{+}^{M}$ such that $\dot{\omega}_{\{j, k\}}=\omega(j, k)$ and $\bar{\omega}_{l}=\omega(j, k)_{j}$. Let $\tilde{R} \in \mathcal{R}^{M}$ be such that $\bar{R}_{\{j, k\}}=R_{\{j, k\}\}}$ and $p\left(\bar{R}_{l}\right)=\bar{\omega}_{l}$. Let $\bar{e}=(\bar{R}, \bar{\omega}) \in$ $\mathcal{E}^{M}$. By individual rationality, $\varphi_{l}(\bar{e})=p\left(\bar{R}_{l}\right)=\bar{\omega}_{l}$. Thus, $\bar{\omega}(j, k)=\omega(j, k)$. Since $\bar{R}_{\{j, k\}}=R_{\{j, k\}}$ and $\bar{\omega}(j, k)=\omega(j, k)$, by bilateral consistency, it follows that $\varphi(\bar{e})_{\{j, k)}=$ $\varphi\left(R_{\{j, k\}}, \omega(j, k)\right)=\varphi(e)$. Next, by bilateral consistency, it follows that $\varphi(\bar{e}\rangle_{\{j, l\}}=$ $\varphi\left(\bar{\omega}(j, l), \bar{R}_{\{j, l\}}\right)$. Since $\Delta \varphi_{l}(\bar{e})=0, \bar{\omega}_{j}=\bar{\omega}_{l}=\omega(j, k)_{j}, \varphi_{j}(\bar{e})=\varphi_{j}\left(R_{\{j, k\}}, \omega(j, k)\right)=$ $\varphi_{j}(e)$, and (4.18),

$$
\begin{aligned}
\bar{\omega}(j, l)_{j} & =\bar{\omega}_{j}+\frac{1}{2}\left(\Delta \varphi_{j}(\bar{e})+\Delta \varphi_{l}(\bar{e})\right) \\
& =\bar{\omega}_{j}+\frac{1}{2} \Delta \varphi_{j}(\bar{e}) \\
& =\bar{\omega}_{j}+\frac{1}{2}\left(\varphi_{j}(\bar{e})-\bar{\omega}_{j}\right) \\
& =\frac{1}{2} \omega(j, k)_{j}+\frac{1}{2} \varphi_{j}(e)<\varphi_{j}(e) .
\end{aligned}
$$

By individual rationality, $\bar{\omega}(i, l)_{j} \leq p\left(R_{j}\right)<\varphi_{i}(e)$. Let $l=l^{\mathbb{1}}$ and $e^{1}=\left(\bar{R}_{\left\{j, l^{1}\right\}}, \bar{\omega}\left(i, l^{1}\right)\right)$. By adding non-traders in this manner we obtain a sequence of economies $\left\{e^{t}\left(j, l^{t}\right)\right\}_{t \in \mathbb{N}}$ such that for all $t \in \mathbb{N}$,

$$
\begin{aligned}
\omega^{t}\left(j, l^{t}\right)_{i} & \leq p\left(R_{j}\right)<\varphi_{j}(e) \text { and } \\
\omega^{t}\left(j, l^{t}\right)_{i} & =\left(\frac{1}{2}\right)^{t} \omega(j, k)_{j}+\sum_{q=1}^{t}\left(\frac{1}{2}\right)^{q} \varphi_{j}(e) \\
& =\left(\frac{1}{2}\right)^{t} \omega(j, k)_{j}+\left(1-\left(\frac{1}{2}\right)^{t}\right) \varphi_{j}(e) .
\end{aligned}
$$


This obviously yields a contradiction for $t \in \mathbb{N}$ large enough. Hence, for all $N \in \mathcal{N}$ such that $|N| \geq 3$ and all $e=(R, \omega) \in \mathcal{E}^{N}$ it follows that $\varphi(e)=U^{r}(e)$.

Case 1.2: $|N|=2$. Hence, $N=\{j, k\}$. We enlarge the economy $(R, \omega)$ by a nontrader $l \neq j, k$. Let $M=\{j, k, h\}$ such that $l \notin\{j, k\}$ and consider the endowment vector $\bar{\omega} \in \mathbb{R}_{+}^{M}$ such that $\bar{\omega}_{\left\{j_{i} k\right\}}=\omega$ and $\bar{\omega}_{l}=0$. Let $\bar{R} \in \mathcal{R}^{M}$ be such that $\bar{R}_{\left\{j_{k}, k\right\}}=R$ and $\bar{R}_{l}$ such that $p\left(\bar{R}_{l}\right)=0$. Let $\bar{e}=(\bar{R}, \bar{\omega}) \in \mathcal{E}^{M}$. By Case $\mathbb{1}, \varphi(\bar{e})=U^{r}(\vec{e})$. Note that $e=\left(\bar{R}_{\{j, k\}}, \bar{\omega}(j, k)\right)$ and $U^{r}(\bar{e})_{\{j, k\}}=U^{r}(e)$. Then, by bilateral consistency, it follows that $\varphi(\bar{e})_{\{j, k j}=\varphi(e)$. Hence, $p\left(R_{j}\right)<\varphi_{j}(e)=\varphi_{j}(\bar{e})=U_{j}^{r}(\bar{e})=U_{j}^{r}(e)=p\left(R_{j}\right)$. This is a contradiction.

Case 2: $z(e)>0$. Similar to Case 1 .

Since individual rationality and strong one-sided endowment-monotonicity imply Pareto optimality (see Lemma 4.19), Theorem 4.9 implies the following characterization of the uniform reallocation rule. This characterization can be seen as an extension of Sönmez's characterization of the uniform allocation rule by individual rationality from equal division, one-sided resource-monotonicity, and consistency (see Theorem 2.14).

Corollary 4.11 The uniform rule $U^{r}$ is the only rule that satisfies individual rationality, strong one-sided endowment-monotonicity, and bilateral consistency.

Proof. The uniform rule $U^{r}$ satisfies individual rationality, strong one-sided endowmentmonotonicity, and bilateral consistency. To prove the remaining part of the corollary let $\varphi$ be a rule that satisfies the properties listed in the corollary. By Lemma 4.19, $\varphi$ is Pareto optimal. Then, by Theorem $4.9, \varphi=U^{r}$.

Remark 4.4 Theorem 4.9 and Corollary 4.11 remain true if we restrict the set of potential agents to a subset of $\mathbb{N}$ that contains at least three agents. For a potential set of agents that contains only two agents the theorem and the corollary do not hold, because in that situation bilateral consistency has no bite.

The following lemma is an extension of a result of Dagan (1996, Lemma 2) for allocation rules.

Lemma 4.21 Let the rule $\varphi$ satisfy Pareto optimality, weak no-envy, and bilateral consistency. Then, on the domain of two-agent economies, $\varphi$ satisfies demand-onliness.

The proof of Lemma 4.21 is similar to the proof of Dagan's result (1996, Lemma 2).

Proof. Let $\varphi$ be a rule that satisfies Pareto optimality, weak no-envy, and bilateral consistency. Let $N \in \mathcal{N}, e=(R, \omega) \in \mathcal{E}^{N}$, and $\bar{e}=(\tilde{R}, \bar{\omega}) \in \mathcal{E}^{N}$ be such that for all $i \in N, d_{i}(e)=d_{i}(\tilde{e})$. In order to prove demand-onliness we have to show that for all $i \in N, \Delta \varphi_{i}(e)=\Delta \varphi_{i}(\bar{e})$.

Case 1: $z(e) \leq 0$. Assume, without loss of generality, that $N=\{1,2\}$. Let $N^{\prime}=\{3,4\}$ and define $\epsilon^{\prime}=\left(R^{\prime}, \omega^{\prime}\right) \in \mathcal{E}^{N^{*}}$ such that for all $i \in N, \omega_{i+n}^{\prime \prime}=\omega_{i}$ and 
$p\left(R_{i+n}^{\prime}\right)=p\left(\bar{R}_{i}\right)$. Clearly $z\left(e^{\prime}\right)<0$. Consider the merged economy $\bar{e}=(\tilde{R}, \bar{\omega})=$ $\left(\left(R, R^{\prime}\right),\left(\omega, \omega^{\prime}\right)\right) \in \mathcal{E}^{\text {NuN }}$. Weak no-envy and same-sidedness imply for all $i \in N$, $\Delta \varphi_{i}(\tilde{e})=\Delta \varphi_{i+2}(\tilde{e})$

Suppose all $i \in N$ leave the economy $\tilde{e}$. Then, $\omega(1,2)=\omega$. Hence, by bilateral consistency, for all $i \in N, \Delta \varphi_{i}(e)=\Delta \varphi_{i}(\tilde{e})=\Delta \varphi_{i+2}(\tilde{e})$.

Suppose all $i \in N$ leave the economy $\tilde{e}$. Then, $\tilde{\omega}(3,4)=\omega^{\prime}$. Hence, by bilateral consistency, for all $i \in N^{\prime}, \Delta \varphi_{i}\left(e^{\prime}\right)=\Delta \varphi_{i}(\bar{e})=\Delta \varphi_{i-2}(\tilde{e})$.

Summarizing, wre have for all $i \in N, \Delta \varphi_{i}(e)=\Delta \varphi_{i+2}\left(e^{\prime}\right)$.

By applying the same argument to the economies $\bar{e}$ and $e^{\prime}$ instead of $e$ and $e^{\prime}$ it follows that for all $i \in N, \Delta \varphi_{i}(\bar{e})=\Delta \varphi_{i+2}\left(e^{\prime}\right)$. Hence, for all $i \in N, \Delta \varphi_{i}(e)=$ $\Delta \varphi_{i+2}\left(e^{\prime}\right)=\Delta \varphi_{i}(\vec{e})$.

Case 2: $z(e)>0$. Similar to Case 1 .

Remark 4.5 Lemma 4.21 holds if we restrict the set of potential agents to a subset of Nhat contains at least four agents.

The next characterization in this section is obtained by extending a result of Dagan (1996, Lemma 2) to reallocation rulles and applying bilateral consistency and Corollary 4.8. This characterization can be seen as an extension of Dagan's characterization of the uniform allocation rule by Pareto optimality, bilateral consistency, and no-envy (see Theorem 2.11).

Theorem 4.10 The uniform rule $U^{r}$ is the only rule that satisfies Pareto optimality, weak no-envy, and bilateral consistency.

Proof. The uniform rule $U^{r}$ satisfies Pareto optimality, weak no-envy, and bilateral consistency.

To prove the remaining part of the theorem let $\varphi$ be a rule that satisfies the properties listed in the theorem. Then, by Lemma 4.21 and bilateral consistency it follows that $\varphi$ satisfies demand-onliness. Hence, by Corollary $4.8, \varphi=U^{r}$.

Remark 4.6 Lemma 4.21 and Theorem 4.10 remain true if we restrict the set of potential agents to a subset of $\mathbb{N}$ that contains at least four agents. For a potential set of agents that contains only three agents it is not clear whether Lemma 4.21 holds. If the potential set of agents only contains two agents, then the theorem does not hold, because in that situation bilateral consistency has no bite.

Remark 4.7 In Thomson (1994b) and Dagan (1996) results similar to those described in Theorem 4.9 and Theorem 4.10 are stated for allocation rules. Thomson includes a continuity condition in his characterizations (1994b, Theorem 1, Theorem 2), besides bilateral consistency, individual rationality from equal division or no-envy respectively. Dagan proves that the results of Thomson remain true without continuity for allocation problems with at least four agents (1996, Theorem 2, Theorem 3). Now, "translating" 
the steps of the proofs $\mathrm{s}^{25}$ in Dagan's characterizations into the reallocation setting is almost sufficient to get alternative proofs of Theorem 4.9 and Theorem 4.10. The argument of converse consistency (see Dagan 1996, Lemma 4), which completes the proofs of the characterizations, however, has no equivalent in the reallocation setting. By assuming that a rule satisfying the characterizing properties does not equal the uniform reallocation rule, and using bilateral consistency, a contradiction which completes the alternative proofs is easily derived.

The last, and hopefully not least, characterization we present here is the following. The uniform rule $U^{r}$ is the only rule that satisfies Pareto optimality, endowment strategy-proofness, bilateral consistency, and the dummy property.

Theorem 4.11 The uniform rule $U^{r}$ is the only rule that satisfies Pareto optimality, bilateral consistency, endowment strategy-proofness, and the dummy property.

Proof. The uniform rule $U^{r}$ satisfies the properties listed in the theorem.

To prove the remaining part of the theorem let $\varphi$ be a rule that satisfies the properties listed in the theorem. By Pareto optimality, endowment strategy-proofness, and Corollary 4.3 , the rule $\varphi$ is bounded by endowments and peaks on the domain of economies that are either balanced or that have excess demand. Hence, by Theorem 4.8 , for all $N \in \mathcal{N}^{\top}$ and all $e=(R, \omega) \in \mathcal{E}^{N}$ such that $z(e) \geq 0, \varphi(e)=U^{r}(e)$.

Next, we prove that the rule $\varphi$ is bounded by endowments and peaks on the domain of economies that have excess supply. Then, by Theorem 4.8 , we are done.

Let $N \in \mathcal{N}$ and $e=(R, \omega) \in \mathcal{E}^{N}$ such that $z(e)<0$. By same-sidedness, for all $i \in N, \varphi_{i}(e) \geq p\left(R_{i}\right)$.

Let $i \in S(e)$ and consider $\omega^{\prime} \in \mathbb{R}_{+}^{N}$ such that $\omega_{k}^{t}=\omega_{k}$ for all $k \in N \backslash\{i\}$ and $e_{i}^{\prime}=\max \left\{p\left(R_{i}\right), \omega_{i}-s(e)+d(e)\right\}$. Let $e^{\prime}=\left(R, \omega^{\prime}\right) \in \mathcal{E}^{N}$. So, at $e^{\prime}$ agent $i$ is either a non-trader or a supplier and demand equals supply. Therefore, $\varphi_{i}\left(e^{\prime}\right)=p\left(R_{i}\right)$. Hence, $\Delta \varphi_{i}\left(e^{\prime}\right) \leq 0$. Since $\omega^{\prime}$ is an $i$-deviation from $\omega$ by withholding, by Lemma 4.8 it follows that $\Delta \varphi_{i}(e) \leq \Delta \varphi_{i}\left(e^{i}\right) \leq 0$. Hence, for all $i \in S(e), \omega_{i} \geq \varphi_{i}(e) \geq p\left(R_{i}\right)$.

Let $j \in \bar{D}(e)$ and suppose, in contradiction to boundedness by endowments and peaks, that there exist such that $\varphi_{j}(e)>p\left(R_{j}\right)$. Then, by feasibility, there exists $i \in S(e)$ and, by the previous step of the proof, $\Delta \varphi_{i}(e) \leq 0$. By bilateral consistency, $\varphi_{j}\left(R_{\{i, j\}}, \omega(i, j)\right)=\varphi_{j}(e)$.

Since $\Delta \varphi_{i}(e) \leq 0, \omega(i, j)_{j}<\varphi_{j}(e)$. We already proved that suppliers obtain an allotment between their peak and their endowment. Therefore, $j$ cannot be a supplier and $\omega(i, j)_{i} \leq p\left(R_{i}\right)<\varphi_{i}(e)$.

Now, similarly as in the proof of Theorem 4.9 , by introducing a sequence of nontraders $\left\{l^{t}\right\}_{t \in \mathbb{N}}$ we obtain a sequence of economies $\left\{e^{t}\left(j, l^{t}\right)\right\}_{t \in \mathbb{N}}$ such that for all $t \in \mathbb{N}$,

$$
\begin{aligned}
\omega^{t}\left(j, l^{t}\right)_{i} & \leq p\left(R_{j}\right)<\varphi_{j}(e) \text { and } \\
\omega^{t}\left(j, l^{t}\right)_{i} & =\left(\frac{1}{2}\right)^{t} \omega(j, k)_{j}+\sum_{q=1}^{t}\left(\frac{1}{2}\right)^{q} \varphi_{j}(e)
\end{aligned}
$$

${ }^{25}$ Dagan (1996), Lemmas 2,3,5 and 6 can be proved in their "reallocation version". 


$$
=\left(\frac{1}{2}\right)^{t} \omega(j, k)_{j}+\left(1-\left(\frac{1}{2}\right)^{t}\right) \varphi_{j}(e)
$$

This obviously yields a contradiction for $t \in \mathbb{N}$ large enough.

A summary of the characterizations of the uniform reallocation rule $U^{r}$ by Pareto optimality or individual rationality, consistency properties, etc. can be found in Table 4.3. For a comparison with corresponding characterizations of the uniform allocation rule $U$ we refer to Tables 2.3 and 2.4 .

\begin{tabular}{|l||c|c|c|c|c|c|}
\hline & Th & Co & Th & Co & Th & Th \\
& 4.8 & 4.10 & 4.9 & 4.11 & 4.10 & 4.11 \\
\hline \hline Pareto optimality & $\times$ & $\times$ & $\times$ & & $\times$ & $\times$ \\
\hline bilaterd consistency & $\times$ & $\times$ & $\times$ & $\times$ & $\times$ & $\times$ \\
\hline $\begin{array}{l}\text { boundedness by } \\
\text { endowments and peaks }\end{array}$ & $\times$ & & & & & \\
\hline dummy property & & $\times$ & & & & $\times$ \\
\hline individual rationality & & & $\times$ & $\times$ & & \\
\hline $\begin{array}{l}\text { one-sided endowment- } \\
\text { monotonicity }\end{array}$ & & $\times$ & & & & \\
\hline $\begin{array}{l}\text { strong one-sided endowment- } \\
\text { monotonicity }\end{array}$ & & & & $\times$ & & \\
\hline weak no-envy & & & & & $\times$ & \\
\hline endowment strategy-proofness & & & & & & $\times$ \\
\hline
\end{tabular}

Table 4.3: Characterizations of the uniform rule $U^{\text {r }}$ by Pareto optimality or individual rationality, bilateral consistency, etc.

\subsection{Proofs of independence}

First, we discuss the logical independence of the properties in the characterizations given in Section 4.3. (see Table 4.1). We introduce the following reallocation rules.

Example 4.5 The no-trade rule $\varphi^{0}$ is defined as follows. For all $N \in \mathcal{N}$ and $e=$ $(R, \omega) \in \mathcal{E}^{N}, \varphi^{0}(e):=\omega$.

Example 4.6 The hierarchical rule $\varphi^{h}$ is defined as follows. ${ }^{26}$ In case of excess demand, the rule satiates all suppliers. Then, the demander with the lowest index is "served" as well as possible, i.e., he receives his peak if that is possible and the total supply

\footnotetext{
${ }^{26}$ The hierarchical rule is often called serial, or lexicographic, dictatorship.
} 
otherwise. If there is something left, the demander with the second lowest number is served, etc. Let $N \in \mathcal{N}$ and $e=(R, \omega) \in \mathcal{E}^{N}$. If $z(e) \geq 0$, then for all $i \in N$,

$$
\varphi_{i}^{h}(e):= \begin{cases}p\left(R_{i}\right) & \text { if } i \notin D(e), \\ \min \left\{p\left(R_{i}\right), \omega_{i}+s(e)-\sum_{\substack{D(j) \\ j<i}} \Delta \varphi_{j}^{h}(e)\right\} & \text { otherwise. }\end{cases}
$$

If $z(e) \leq 0$, then $\varphi^{h}(e)$ is defined similarly.

Example 4.7 The following maximally satiating rule $\varphi^{\max }$ satiates as many agents as possible. Let $N \in \mathcal{N}$ and $e=(R, \omega) \in \mathcal{E}^{N}$. If $e$ is in excess demand, then the total supply is distributed among the demanders as follows. First minimal demands are satiated uniformly. If there is some supply left, then the next smallest demands are satiated, and so on. If $z(e) \geq 0$ and, without loss of generality, $D(e)=\left\{1,2, \ldots, d_{k}\right\}$ such that $d_{1}(e)=\ldots=d_{t_{1}}(e)<d_{t_{1}+1}(e)=\ldots=d_{t_{2}}(e)<\ldots<d_{t_{r}}(e)=\ldots=d_{k}(e)$, then for all $i \in N$,

$$
\varphi_{i}^{\max }(e):= \begin{cases}p\left(R_{i}\right) & \text { if } i \notin D(e), \\ \min \left\{p\left(R_{i}\right), \omega_{i}+\frac{1}{t_{s}-t_{s-1}}\left(s(e)-\sum_{j \leq t_{s-1}} \Delta \varphi_{j}(e)\right)\right\} & \text { if } i \in D(e) \\ & \text { and } \\ & t_{s-1}<i \leq t_{s}\end{cases}
$$

If $z(e) \leq 0$, then $\varphi^{\max }(e)$ is defined similarly with respect to smallest supplies.

Example 4.8 The following absorbing agent rule $\varphi^{a b}$ is similar to the allocation rule $\varphi^{a}$ we introduced in Chapter 2. Let $N \in \mathcal{N}$ and $e=(R, \omega) \in \mathcal{E}^{N}$ be such that $z(e)<0$ and $D(e)=\{j\}$. Then, for this economy we obtain $\varphi^{a b}(e)$ from the uniform reallocation $U^{r}(e)$ by letting agent $j$ absorb the amount $a(e):=\min \left\{s(e), r_{j}\left(\omega_{j}\right)-p\left(R_{j}\right)\right\}$ and subtracting this amount uniformly from the uniform allocation shares $U_{i}^{r}(e)$ of the suppliers $i \in N \backslash\{j\}$. In order to formalize this, let $R^{\prime} \in \mathcal{R}$ be a $j$-deviation from $R$ such that $p\left(R_{j}^{\prime}\right)=p\left(R_{j}\right)+a(e)$. Then, $\varphi^{a b}(e):=U^{r}\left(R^{\prime}, U^{r}(e)\right)$.

For all other economies, we apply the uniform reallocation rule. So, for all remaining $N \in \mathcal{N}$ and $e=(R, \Omega), \varphi^{a b}(e):=U^{r}(e)$.

Recall that the reallocation rule $\varphi^{1}$ is defined in Example 4.1, page 73 .

In Table 4.4 we show which of the properties introduced in Section 4.3 are satisfied by the rules introduced above (marked in the table by a " + ") and which are not (marked in the table by a "-"). In the lower part of the table we indicate for each characterization of Section 4.3 which of the properties in the characterization is not satisfied by the rule while all other characterizing properties are satisfied. The entry " " means that more than one characterizing property is not satisfied and the rule is 


\begin{tabular}{|l||c|c|c|c|c|}
\hline \multicolumn{1}{|c|}{ Reallocation rules $\rightarrow$} & $\varphi^{0}$ & $\varphi^{\text {h }}$ & $\varphi^{\text {max }}$ & $\varphi^{a b}$ & $\varphi^{I}$ \\
Properties and abbreviations & & & & & \\
\hline \hline Pareto optimality Po & - & + & + & + & + \\
\hline strategy-proofness sp & + & + & - & - & + \\
\hline endowment strategy-proofness esp & + & + & - & + & + \\
\hline noemwy ne & + & - & - & + & - \\
\hline weak no-envy wne & + & - & - & + & + \\
\hline equal treatment of equals ete & + & - & + & + & + \\
\hline anonymity an & + & - & + & + & + \\
\hline demand-onliness do & + & + & + & - & - \\
\hline reversibility re & + & + & + & - & - \\
\hline peak-onliness po & + & + & + & - & + \\
\hline Theorem 4.1 & Po & ne & $\backslash$ & sp & ne \\
\hline Corollary 4.5 & Po & ete & sp & $\backslash$ & do \\
\hline Corollary 4.6 & Po & an & sp & $\backslash$ & do \\
\hline Theorem 4.2 & Po & ete & esp & re & re \\
\hline Theorem 4.3 & Po & ne & ne & po & ne \\
\hline Corollary 4.8 & Po & wne & wne & do & do \\
\hline
\end{tabular}

Table 4.4: Proofs of independence, Section 4.3

not used in the proof of independence.

Next, we discuss the logical independence of the properties in the characterizations given in Section 4.4. (see Table 4.2). In addition to the previous examples we introduce the following reallocation rules.

Example 4.9 The following rule $\varphi^{\text {at }}$ is a variation of the absorbing agent rule $\varphi^{\text {ab }}$ (see Example 4.8). Let $N \in \mathcal{N}$ and $e=(R, \omega) \in \mathcal{E}^{N}$. If $z(e)<0$ and $D(e)=\{j\}$, then $\tilde{\varphi}^{a b}(e):=\varphi^{a b}(e)$.

If $e$ is a $k$-replica of an econorry $\ddot{e} \in \mathcal{E}^{\bar{N}}$ such that $z(\bar{e})<0$ and $D(\bar{e})=\{j\}$, then $\hat{\varphi}^{a b}(e)$ is a $k$ - replica of $\varphi^{a b}(e)$.

For all other economies, we apply the uniform reallocation rule. So, for all remaining $N \in \mathcal{N}$ and $e=(R, \Omega), \dot{\varphi}^{a b}(e):=\operatorname{Ur}(e)$.

Example 4.10 The rule $\varphi^{+\lambda}$ is defined as follows. Let $N \in \mathcal{N}$ and $e=(R, \omega) \in \mathcal{E}^{N}$. If $e$ is in excess supply, then the social endowment $\Omega=\sum_{N} \omega_{i}$ is divided so that all agents have the same supply with respect to their peaks. So, if $z(e)<0$, then for all $i \in N, \varphi_{i}^{+\lambda}(e):=p\left(R_{i}\right)+\lambda$, where $\lambda \geq 0$ solves $\sum_{N} \varphi_{i}^{+\lambda}(e)=\Omega$.

If $z(e) \geq 0$, then $\varphi^{+\lambda}(e):=U^{r}(e)$.

In Table 4.5 we show which of the properties introduced in Section 4.4 are satisfied by the rules introduced above (marked in the table by $a^{\text {"t }}+$ ) and which are not (marked in the table by $a^{\text {" }}-$ " $)$. In the lower part of the table we indicate for each characterization 
of Section 4.4 which of the properties in the characterization is not satisfied by the rule while all other characterizing properties are satisfied. The extry " means that more than one characterizing property is not satisfied and the rule is not used in the proof of independence. As already mentioned in Section 4.4, we did not succeed in proving the independence of Corollary 4.9 .

\begin{tabular}{|l||c|c|c|c|c|}
\hline $\begin{array}{l}\text { Reallocation rules } \rightarrow \\
\text { Properties and abbreviations } \downarrow\end{array}$ & $\varphi^{0}$ & $\varphi^{h}$ & $\varphi^{a b}$ & $\bar{\varphi}^{a b}$ & $\varphi^{+\lambda}$ \\
\hline \hline Pareto optimality Po & - & + & + & + & + \\
\hline no-envy ne & + & - & + & + & - \\
\hline weak no-envy wne & + & - & + & + & - \\
\hline $\begin{array}{l}\text { one-sided population- } \\
\text { monotonicity pm }\end{array}$ & + & + & - & - & + \\
\hline $\begin{array}{l}\text { one-sided replacement- } \\
\text { domination rd }\end{array}$ & + & + & + & - & + \\
\hline $\begin{array}{l}\text { one-sided endowment- } \\
\text { monotonicity em }\end{array}$ & - & + & - & - & + \\
\hline $\begin{array}{l}\text { strong one-sided endowment- } \\
\text { monotonicity sem }\end{array}$ & - & - & - & + & + \\
\hline replication-invariance ri & + & + & + & + & - \\
\hline individual rationality ir & Po & wne & pm & pm & wne \\
\hline \hline Theorem 4.4 & Po & $\downarrow$ & ri & rd & ne \\
\hline Theorem 4.5 & Po & wne & em & em & wne \\
\hline Theorem 4.6 & sem & wne & sem & $\backslash$ & $\backslash$ \\
\hline Corollary 4.9 & & & & + \\
\hline
\end{tabular}

Table 4.5: Proofs of independence, Section 4.4

Finally, we discuss the logical independence of the properties in the characterizations given in Section 4.5. (see Table 4.3). In addition to the previous examples, we introduce the following reallocation rule.

Example 4.11 The rule $\varphi^{s}$ is defined as follows. Let $N \in N$ and $e=(R, \omega) \in \mathcal{E}^{N}$." If $e$ is in excess supply, then all agents except the agent(s) with maximal supply are satiated. Feasibility is adjusted on the account of the agent(s) with maximal supply, $S_{\max }(e)=\arg \max _{i \in S(e)} s_{i}(e)$. So, if $z(e)<0$, then for all $i \in N$,

$$
\varphi_{i}^{s}(e):= \begin{cases}p\left(R_{i}\right) & \text { if } i \notin S_{\max }(e) \text { and } \\ \omega_{i}+\lambda & \text { if } i \in S_{\max }(e)\end{cases}
$$

where $\lambda \geq 0$ solves $\sum_{N} \varphi_{i}^{s}(e)=\sum_{N} w_{i}$.

For all other economies, we apply the uniform reallocation rule. So, if $z(e) \geq 0$, then $\varphi^{s}(e):=U^{r}(e)$. 


\begin{tabular}{|c|c|c|c|c|c|}
\hline $\begin{array}{l}\text { Reallocation rules } \rightarrow \\
\text { Properties and abbreviations }\end{array}$ & $\varphi^{0}$ & $\varphi^{h^{2}}$ & $\varphi^{+\lambda}$ & $\varphi^{a b}$ & $\varphi^{g}$ \\
\hline Pareto optimality Po & - & +4 & + & + & + \\
\hline bilateral consistency bc & + & - & + & - & + \\
\hline $\begin{array}{l}\text { boundedness by endowments } \\
\text { and peaks bep }\end{array}$ & + & + & - & - & - \\
\hline dummy property $\mathrm{dp}$ & + & + & - & + & + \\
\hline individual rationality ir & + & + & - & + & - \\
\hline $\begin{array}{l}\text { one-sided endowment- } \\
\text { monotonicity em }\end{array}$ & + & + & + & - & - \\
\hline $\begin{array}{l}\text { strong ane-sided endowment- } \\
\text { monotonicity sem }\end{array}$ & - & + & + & - & - \\
\hline demand-onliness do & + & + & + & $\overline{-}$ & + \\
\hline weak no-envy wne & + & - & - & + & - \\
\hline $\begin{array}{l}\text { endowment strategy- } \\
\text { proofness esp }\end{array}$ & + & + & $t$ & + & - \\
\hline Theorem 4.8 & $\mathrm{Po}$ & $\mathrm{bc}$ & bep & bep & bep \\
\hline Corollary 4.10 & Po & $\mathrm{bc}$ & $\mathrm{dp}$ & $T$ & em \\
\hline Theorem 4.9 & Po & $\mathrm{bc}$ & $i x$ & $\mathrm{bc}$ & ir \\
\hline Corollary 4.11 & sem & bc & ir & 1 & 1 \\
\hline Theorem 4.10 & Po & 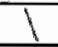 & wne & $\mathrm{bc}$ & wne \\
\hline Theorem 4.11 & $\mathrm{Po}$ & $\mathrm{bc}$ & $\mathrm{dp}$ & $\mathrm{bc}$ & esp \\
\hline
\end{tabular}

Table 4.6: Proofs of independence, Section 4.5

In Table 4.6 we show which of the properties introduced in Section 4.5 are satisfied by the rules introduced above (marked in the table by a " + ") and which are not (marked in the table by $a$ " - "). In the lower part of the table we indicate for each characterization of Section 4.5 which of the properties in the characterization is not satisfied by the rule while all other characterizing properties are satisfied. "The entry " means that more than one characterizing property is not satisfied and the rule is not used in the proof of independence. 


\section{Chapter 5}

\section{Model variations ${ }^{1}$}

As in Chapter 4, we study the problem of reallocating the individual endowments of some perfectly divisible commodity among a group of agents with single-peaked preferences. For corresponding literature, a more detailed explanation of the model, and the connection between allocation and reallocation rules and their properties, we refer the reader to Chapter 4. The aim of this chapter is to study some variations of the model introduced in Chapter 4. We indicate whether the results derived in Chapter 4 remain true under the model variations at hand. Furthermore, we show how some of the results we derived in Chapter 4 can be adjusted to these models.

This chapter is organized as follows. In Section 5.1 the model is extended to include arbitrary, possibly negative, individual endowments and reallocations. Furthermore, the agents' preferences are defined on the whole real line with possibly negative peaks. Hence, in this model, we include the possibility of debts. For this model, we characterize the uniform reallocation rule by Pareto optimality, strategy-proofness, and a equal treatment of equals condition (see Theorem 5.1).

In Section 5.2, we still consider preferences that are defined on the whole real line, but restrict peaks, individual endowments, and reallocations to be non-negative. Hence, debts are not allowed any more although agents still have preferences over debts. For this model, we characterize the uniform reallocation rule by Pareto optimality, strategyproofness, an adjusted equal treatment of equals condition, and a reversibility condition (see Theorem 5.2).

Finally, in Section 5.3, we discuss the following three model variations concerning the preference relations, the individual endowments and reallocations, and the potential set of agents: discontinuity of preferences, boundedness of individual endowments and reallocations, and a finite set of potential agents.

For the model variations mentioned above, most results we derived in Chapter 4 remain true. For each model variation, we indicate which results of Chapter 4 remain true and which do not remain true.

\footnotetext{
${ }^{1}$ This chapter is mainly based on Kllaws, Peters, and Storcken (1995a) and Klaus, Peters, and Storcken (1995b).
} 


\subsection{Strategy-proof reallocation with debts}

In the reallocation model we consider in Chapter 4 the agents' preferences are defined on the ron-negative real line and individual endowments and allotments are bounded from below by zero. This causes an asymmetry between excess demand and excess supply economies we already emphasized in Chapter 4 (see Remark 4.2). In the first model variation we consider in this chapter, we assume that the agents' preferences are single-peaked and defined over the whole real line. Furthermore, individual endowments, peaks, and shares may be negative. Hence, in this model, we include the possibility of debts. In order to keep this chapter as self-contained as possible; we first give a complete description of this reallocation model with debts.

There is an infinite population of potential agents, indexed by the natural numbers $\mathbb{N}$. Each agent $i \in \mathbb{N}$ is described in terms of an individual endowment $w_{i} \in \mathbb{R}$ of an infinitely divisible commodity, and a continuous and single-peaked preference relation $R_{i}$ defined over the real numbers $\mathbb{R}$. Single-peakedness of $R_{i}$ means that there exists a point $p\left(R_{i}\right) \in \mathbb{R}$, called the peak of agent $i$, with the following property: for all $x, y \in \mathbb{R}$ with $x<y \leq p\left(R_{i}\right)$ or $x>y \geq p\left(R_{i}\right)$, we have $y P_{i} x$.

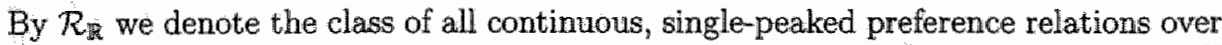
$\mathbb{R}$. By $\mathcal{N}$ we denote the class of non-empty and finite subsets of $\mathbb{N}$. For $N \in \mathcal{N}, \mathcal{R} \mathbb{R}_{\mathbb{R}}^{N}$ denotes the set of (preference) profiles $R=\left(R_{i}\right)_{i \in N}$ such that for all $i \in N, R_{i} \in \mathcal{R}_{\mathbb{R}}$. For profile $R \in \mathcal{R}_{\mathbb{R}}^{N}, p(R)=\left(p\left(R_{i}\right)\right)_{i \in N}$ denotes the vector of peaks.

Throughout this section, an economy is formalized as follows. Let $N \in \mathcal{N}$ and $\omega=\left(\omega_{i}\right)_{i \in N} \in \mathbb{R}^{N}$ be a vector of individual endowments that have to be reallocated among the agents in $N$ with profile $R \in \mathcal{R}_{\mathbb{R}}^{N}$. We call a pair $e=(R, \omega) \in \mathcal{R} \mathbb{\mathbb { R }} \times \mathbb{R}^{N}$ an economy. Let $\mathcal{E}_{1}^{N}=\mathcal{R}_{\mathbb{R}}^{N} \times \mathbb{R}^{N}$ be the class of all these economies. A feasible allocation for $e=(R, \omega) \in \mathcal{E}_{1}^{N}$, or a reallocation for short, is a vector $x \in \mathbb{R}^{N}$ such that $\Sigma_{N} x_{i}=\sum_{N} \omega_{i}$. A reallocation rule $\varphi_{1}$ or a rule for short, is a function that assigns to every $N \in \mathcal{N}$ and every $e=(R, \omega) \in \mathcal{E}_{1}^{N}$ a reallocation, denoted by $\varphi(e)$.

Let $N \in \mathcal{N}$ and $e=(R, \omega) \in \mathcal{E}_{1}^{N}$. Given $i \in N$, we call $\varphi_{i}(e)$ the allotment of agent $i$ and $\Delta \varphi_{i}(e):=\varphi_{i}(e)-\omega_{i}$ his allotment change. Agent $i$ is satiated at $\varphi(e)$ if $p\left(R_{i}\right)=\varphi_{i}(e)$ and non-satiated at $\varphi(e)$ if $p\left(R_{i}\right) \neq \varphi_{i}(e)$.

We call agent $i \in N$ a demander if his endowment is strictly less than his peak: he "demands" $p\left(R_{i}\right)-\omega_{i}$ units of the commodity. For all $i \in N$, we denote this demand by $d_{i}(e)$ and the set of demanders by $D(e)$. We call agent $i \in N$ a supplier if his endowment is strictly greater than his peak: he wants to "supply" $\omega_{i}-p\left(R_{i}\right)$ units of the commodity. For all $i \in N$, we denote this supply by $s_{i}(e)$ and the set of suppliers by $S(e)$. We call agent $i \in N$ a non-trader if his endowment is equal to his peak: he favors no trade, $i_{i}$.., $d_{i}(e)=s_{i}(e)=0$. Let $d(e):=\sum_{i \in D(e)} d_{i}(e)$ denote total demand and $s(e):=\sum_{i \in S(e)} s_{i}(e)$ total supply. Furthermore, let $z(e):=\sum_{i \in N} d_{i}(e)$ denote excess demand. The latter may be positive, zero, or negative. If it is zero, then $e$ is balanced. If it is positive, then $e$ is an economy with excess demand. If it is negative, then $e$ is an economy with excess supply. Note that for all $i \in N, d_{i}(e)=-s_{i}(e)$ and $z(e)=d(e)-s(e)$. 
An example of a reallocation rule that satisfies many desirable properties is the uniform (reallocation) rule we extensively studied in Chapter 4 . The definition for the reallocation model with debts is similar to the definition introduced for the original model of Chapter 4 (see Section 42 , page 64).

Uniform rule $U^{r}:$ For all $N \in \mathcal{N}$, all $e=(R, \omega) \in \mathcal{E}_{1}^{N}$, and all $j \in N$;

$$
U_{j}^{r}(e):= \begin{cases}\min \left\{p\left(R_{j}\right), \omega_{j}+\lambda\right\} & \text { if } z(e) \geq 0 \\ \max \left\{p\left(R_{j}\right), \omega_{j}+\lambda\right\} & \text { if } z(e) \leq 0\end{cases}
$$

where $\lambda \in \mathbb{R}$ solves $\sum_{N} U_{i}^{r}(e)=\sum_{N} \omega_{i}$.

So, agents are either satiated or receive the same (maximal or minimal) allotment change.

Let $\varphi$ be a rule. Similar to Section 43 , we are interested in the conditions Pareto optimality (see Section 4.3, page 63) and strategy-proofness (see Section 4.3, page 66).

A stendard requirement for rules is Pareto optimality: a reallocation assigned by the rule cannot be changed in such a way that no agent is worse off and some agent is better off.

Pareto optimality: For all $N \in \mathcal{N}$ and all $e=(R, \omega) \in \mathcal{E}_{1}^{N}$, there is no reallocation $x \in \mathbb{R}^{N}$, such that for all $i \in N, x_{i} R_{i} \varphi_{i}(e)$, and for some $j \in N, x_{j} P_{j} \varphi_{j}(e)$.

Similarly as before it follows easily that a rule is Pareto optimal if and only if it is same-sided, that is: for all $N \in \mathcal{N}$ and all $e=(R, \omega) \in \mathcal{E}_{1}^{N}$, either [for all $i \in N$, $\left.\varphi_{i}(e) \leq p\left(R_{i}\right)\right]$, or [for all $\left.i \in N, \varphi_{i}(e) \geq p\left(R_{i}\right)\right]$.

Strategy-proofness ensures that no agent ever benefits from misrepresenting his preferences. Before we give the formal definition, we repeat some notation.

Let $N, M \in \mathcal{N}$ be such that $N \subseteq M$. Let $M \backslash N=\{i \in M \| i \notin N\}$. For $R \in R_{R}^{M}$ the restriction $\left(R_{i}\right)_{i \in N} \in \mathcal{R}_{\mathbb{R}}^{N}$ of $R$ to $N$ is denoted by $R_{N}$. Let $R, \bar{R} \in \mathcal{R}_{\mathbb{R}}^{N}, i \in N$, and $R_{N \backslash\{i\}}=\bar{R}_{N \backslash\{i\}}$. Then, we call $\bar{R}$ an $i$-deviation from $R$.

Strategy-proofness: For all $N \in \mathcal{N}$, all $e=(R, \omega) \in \mathcal{E}_{1}^{N}$, all $\bar{e}=(\bar{R}, \omega) \in \mathcal{E}_{1}^{N}$, and

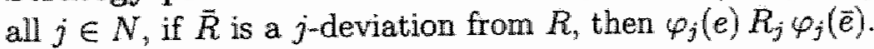

It is easy to check that the uniform rule $U^{r}$ satisfies Pareto optimality and strategy proofness (see Lemma 4.3).

Next, similar to Section 4.3, we prove some implications of Pareto optimality and strategy-proofness. First we state that Pareto optimality and strategy-proofness imply own-peak monotonicity (see Section 4.3, page 67): an agent's allotment is monotonic with respect to his peak. 
Own-peak monotonicity: For all $N \in \mathcal{N}$, all $e=(R, \omega) \in \mathcal{E}_{1}^{N}$, all $\bar{e}=(\vec{R}, \omega) \in \mathcal{E}_{1}^{N}$, and all $j \in N$, if $\vec{R}$ is a $j$-deviation from $R$ and $p\left(R_{j}\right) \leq p\left(\widetilde{R}_{j}\right)$, then $\varphi_{j}(e) \leq \varphi_{j}(\bar{e})$.

Lemma 5.1 Let the rule $\varphi$ satisfy Pareto optimality and strategy-proofness. Then $\varphi$ satisfies own-peak monotonicity.

Proof. Similar to the proof of Lemma 4.4 .

An immediate, but important, consequence of own-peak monotonicity of a rule $\varphi$ is Individual peak-onliness: if an agent changes his preference without changing his peak, his allotment does not change.

Individual peak-onliness: For all $N \in \mathcal{N}$, all $e=(R, \omega) \in \mathcal{E}_{1}^{N}$, all $\bar{e}=(\bar{R}, \omega) \in \mathcal{E}_{1}^{N}$, and all $j \in N$, if $\widetilde{R}$ is a $j$-deviation from $R$ and $p\left(R_{j}\right)=p\left(\ddot{R}_{j}\right)$, then $\varphi_{j}(e)=\varphi_{j}(\bar{e})$.

Next, we show that Pareto optimality and strategy-proofness imply uncompromisingness, a bounded invariance property (see Section 4.3, page 68): if an agent's peak differs from the allotment assigned by the rulle, then his allotment does not change if he unilaterally deviates from his original preference relation in such a way that his new peak and his original peak are at the same side of the allotment.

Uncompromisingness: For all $N \in \mathcal{N}$, all $e=(R, \omega) \in \mathcal{E}_{1}^{N}$, all $\vec{e}=(\bar{R}, \omega) \in \mathcal{E}_{1}^{N}$, and all $j \in N$, if $\bar{R}$ is a $j$-deviation from $R$ and either $\left[p\left(R_{j}\right)>\varphi_{j}(e)\right.$ and $\left.p\left(\bar{R}_{j}\right) \geq \varphi_{j}(e)\right]$ or $\left[p\left(R_{j}\right)<\varphi_{j}(e)\right.$ and $\left.p\left(\bar{R}_{j}\right) \leq \varphi_{j}(e)\right]$, then $\varphi_{j}(e)=\varphi_{j}(\bar{e})$.

Lemma 5.2 Let the rule $\varphi$ satisfy Pareto optimality and strategy-proofness. Then $\varphi$ satisfies uncompromisingness.

Proof. Let $\varphi$ be a rule that satisfies Pareto optimality and strategy-proofness. Let $N \in$ $\mathcal{N}, e=(R, \omega) \in \mathcal{E}_{1}^{N}, \bar{e}=(\bar{R}, \omega) \in \mathcal{E}_{1}^{N}$, and $j \in N$ be such that $\bar{R}$ is a $j$-deviation from $R$. Since, by Lemma 5.1, $p$ satisfies own-peak monotonicity and therefore individual peak-onliness, we assume, without loss of generality, that $R_{j}$ and $\bar{R}_{j}$ are preference relations that are symmetric around the peaks.

Case 1: $p\left(R_{j}\right)<\varphi_{j}(e)$ and $p\left(\hat{R}_{j}\right) \leq \varphi_{j}(e)$. By same-sidedness, it follows that for all

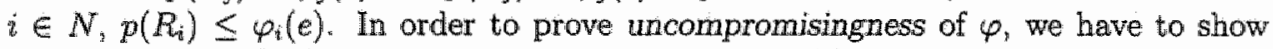
that $\varphi_{j}(e)=\varphi_{j}(\bar{e})$. Suppose, by contradiction, that $\varphi_{j}(e) \neq \varphi_{j}(\bar{e})$.

If $\varphi_{j}(\vec{e})>\varphi_{j}(e)$, then it follows that $p\left(\bar{R}_{j}\right) \leq \varphi_{j}(e)<\varphi_{j}(\vec{e})$. Hence, in contradiction to strategy-proofness, $\varphi_{j}(e) \bar{P}_{j} \varphi_{j}(\bar{e})$.

If $\varphi_{j}(\bar{e})<\varphi_{j}(e)$, then by strategy-proofness, $\varphi_{j}(\tilde{e}) \leq \varphi_{j}(e)-2 p\left(R_{j}\right)$. Hence, by Pareto optimality this implies $p\left(\pi_{j}\right) \leq \varphi_{j}(\bar{e}) \leq \varphi_{j}(e)-2 p\left(R_{j}\right)$. Hence, for all $e^{\prime}=$ $\left(R^{\prime}, \omega\right) \in \mathcal{E}_{1}^{N}$ such that $R^{\prime}$ is a $j$-deviation from $R$ and $p\left(R_{j}^{\prime}\right)>\varphi_{j}(e)-2 p\left(R_{j}\right)$, we have $\varphi_{j}\left(e^{\prime}\right)=\varphi_{j}(e)$. By repeating this argument, each time we double the range of peaks $p\left(R_{j}^{\prime}\right)$ with $\varphi_{j}(e)=\varphi_{j}\left(e^{\prime}\right)$. Finally, $\varphi_{j}(e)=\varphi_{j}(\bar{e})$. 
Case 2: $\quad p\left(R_{j}\right)>\varphi_{j}(e)$ and $p\left(\bar{R}_{j}\right) \geq \varphi_{j}(e)$. Similar to Case 1 .

The repetition argument in the proof of Lemma 5.2 can be avoided by taking a sufficiently asymmetric preference $R_{j}$ instead (see proof of Lemma 4.5 ).

The next property we analyze is no-envy: no agent strictly prefers the allotment change of another agent to his own allotment change. In contrast with the no-envy property of Chapter 4 (see Section 4.3, page 69), the sum of an agent's individual endowment and another agent's allotment change is always feasible in this reallocation model with debts. Hence, the extra assumption of feasibility in the definition of weak no-envy we introduced in Chapter 4 (see Section 4.3, page 70) is automatically satisfied in this reallocation model with debts.

No-envy: For all $N \in \mathcal{N}$, all $e=(R, \omega) \in \mathcal{E}_{1}^{N}$, and all $i, j \in N, \varphi_{j}(e) R_{j}\left(\omega_{j}+\right.$ $\left.\Delta \varphi_{i}(e)\right)$.

The uniform rule $U^{r}$ satisfies no-envy (see Lemma 4.6).

Note that in all results of Chapter 4 no-envy or weak no-envy can be replaced in this model by no-envy as introduced above.

No-envy implies the following equal treatment of equals condition: if the individual endowments and preference relations of two agents are equal up to a translation, then each agent should be indifferent between his own allocation and the translated allocation of the other agent. Before we give the formal definition, we introduce the following notation.

Let $N \in \mathcal{N}$ and $i \in N$. Furthermore, let $R_{i} \in \mathcal{R}_{\mathbb{R}}$ and $\tau \in \mathbb{R}$. Then, the translation of $R_{i}$ by $\tau$, denoted by $R_{i}+\tau$, is defined as follows. For all $\alpha, \beta \in \mathbb{R}, \alpha\left(R_{i}+\tau\right) \beta$ if $(\alpha-\tau) R_{i}(\beta-\tau)$.

Equal treatment of equals: For all $N \in \mathcal{N}$, all $e=(R, \omega) \in \mathcal{E}_{1}^{N}$, all $i, j \in N$, and all $\tau \in \mathbb{R}$, if $R_{j}=R_{i}+\tau$ and $\omega_{j}=\omega_{i}+\tau$, then $\varphi_{j}(e) I_{j} \varphi_{i}(e)+\tau$.

If $\varphi$ is Pareto optimal, then equal treatment of equals implies the stronger equality condition $\Delta \varphi_{i}(e)=\Delta \varphi_{j}(e)$.

Since equal treatment of equals introduced above and the equal treatment of equals condition of Chapter 4 (see Section 4.3, page 72) are introduced for different reallocation models, they are not directly comparable. However, one can easily adjust the equal treatment of equals condition of Chapter 4 to this model. Then, equal treatment of equals we introduced above is a weaker condition (less agents are considered as equals).

The main result of this section is the following characterization of the uniform rule $U^{r}$ by Pareto optimality, strategy-proofness, and equal treatment of equals.

Theorem 5.1 The uniform rule $U^{r}$ is on the domain of economies $\bigcup_{N \in \mathcal{N}} \mathcal{E}_{1}^{N}$ the only rule that satisfies Pareto optimality, strategy-proofness and equal treatment of equals. 
Proof. The uniform rule $U^{r}$ satisfies Pareto optimal, strategy-proofness, and equal treatment of equals.

To prove the remaining part of the theorem let $\varphi$ be a rule that satisfies the properties listed in the theorem. By Lemmas 5.1 and 5.2 both $\varphi$ and $U^{r}$ satisfy own-peak monotonicity and uncompromisingness.

Let $N \in \mathcal{N}, e=(R, \omega) \in \mathcal{E}_{1}^{N}$, and suppose, by contradiction, that $\varphi(e) \neq U^{r}(e)$. Since $\varphi$ is Pareto optimal it follows that either $z(e)>0$ or $z(e)<0$.

Case 1: $z(e)>0$. We call a demander $j$ a maximal demander if $j \in \arg \max \left\{d_{i}(e) \|\right.$ $i \in D(e)\}$. By $d_{\max }:=\max \left\{d_{i}(e) \mid i \in D(e)\right\}$ we denote the maximal demand.

Let $m \in \arg \max \left\{d_{i}(e) \mid i \in D(e)\right\}$ and denote the set of maximal demonders that have, up to a translation, the same preference relation as agent $m$ by $D_{\max }^{r}(e)$; $D_{\max }^{\tau}(e):=\left\{i \in D(e) \mid \omega_{i}-\omega_{m}=\tau_{i}\right.$ and $\left.R_{i}=R_{m}+\tau_{i}\right\}$.

Case 1.1: All agents are maximal demanders and have, up to a translation, the same preference relation as agent $m$, i.e., $D_{\max }^{\tau}(e)=N$. Hence, $U^{r}(e)=\omega$ and for all $i \in N, d_{i}(e)=d_{\max }$. Furthermore, for all $i, j \in N$ we have that $R_{j}=R_{i}+\tau_{i, j}$ and $\omega_{j}=\omega_{i}+\tau_{i, j}$, where $\tau_{i, j}=\tau_{j}-\tau_{i}$. Then, by equal treatment of equals it follows that $\varphi_{j}(e) I_{j} \varphi_{i}(e)+\tau_{i, j}$. Hence, by Pareto optimality, for all $i, j \in N, \varphi_{j}(e)=\varphi_{i}(e)+\tau_{i, j}$. By feasibility and same-sidedness, this can only hold if for all $i \in N, \varphi_{i}(e)=\omega_{i}$. Hence, $\varphi(e)=U^{r}(e)$. This is in contradiction to our assumption that $\varphi(e) \neq U^{r}(e)$.

Case 1.2: The set of maximal demanders $D_{\max }^{\tau}(e)$ does not equal the set of agents, i.e., $D_{\max }^{\tau} \subseteq N$.

Since for all $i, j \in D_{\max }^{\tau}(e)$ we have that $R_{j}=R_{i}+\tau_{i, j}$ and $\omega_{j}=\omega_{i}+\tau_{i, j}$, where $\tau_{i, j}=\tau_{j}-\tau_{i}$, by equal treatment of equals and Pareto optimality it follows that $\varphi_{j}(e)=$ $\varphi_{i}(e)+\tau_{i, j}$. Hence, for all $i, j \in D_{\max }^{\tau}(e), \Delta \varphi_{j}(e)=\Delta \varphi_{i}(e)$. Since $\varphi(e) \neq U^{r}(e)$, there exists an agent $j \notin D_{\max }^{\tau}$ such that $\varphi_{j}(e) \neq U_{j}^{r}(e)$.

Let $\ddot{e}=(\vec{R}, \omega) \in \mathcal{E}_{1}^{N}$ such that $\bar{R}$ is a $j$-deviation from $R$ and $\bar{R}_{j}=R_{m}+\tau_{j}$, where $\tau_{j}=\omega_{j}-\omega_{m}$. We consider the following two subcases.

Case 1.2.1: $\quad \varphi_{j}(e)<U_{j}^{r}(e) \leq p\left(R_{j}\right)$. Since $p\left(R_{j}\right) \leq p\left(\bar{R}_{j}\right)$, by uncompromisingness of $\varphi$ it follows that $\varphi_{j}(\bar{e})=\varphi_{j}(e)$. Own-peak monotonicity of $U^{r}$ yields $U_{j}^{r}(e) \leq U_{j}^{r}(\bar{e})$. Thus, $\varphi_{j}(\bar{e})<U_{j}^{r}(\bar{e})$.

Case 1.2.2: $\quad U_{j}^{r}(e)<\varphi_{j}(e) \leq p\left(R_{j}\right)$. Similar to Case 1.2 .1 (exchange the roles of $U^{r}$ and $\varphi$ ) it follows that $\varphi_{j}(\bar{e})>U_{j}^{r}(\bar{e})$.

Thus, for the economy $\bar{e}$ it follows that $\varphi_{j}(\bar{e}) \neq U_{j}^{r}(\bar{e})$. Note that the economy $\bar{e}$ has one maximal demander with a preference relation that is, up to a translation, the same as agent $m$ 's preference relation more than the economy e, i.e., $\left|D_{\max }^{\tau}(\bar{e})\right|=\left|D_{\max }^{\tau}(e)\right|+1$. By Case 1.1, $D_{\max }^{+}(\ddot{e}) \subsetneq N$.

Similarly, proceeding from the economy $\bar{e}$, we consider a unilateral change of an agent's preference relation such that the set of maximal demanders with a preference relation that is, up to a translation, the same as agent $m$ 's preference relation increases by one and the uniform reallocation for this economy is unequal to the reallocation assigned by $\varphi$, etc. Since the set of agents is finite, finally we end up in an economy $e^{\prime}$ with only maximal demanders who have a preference relation that is, up to a translation, 
the same as agent $m^{\prime}$ 's preference relation and $\varphi\left(e^{r}\right) \neq U^{r}\left(e^{r}\right)$. By Case 1.1, this is a contradiction.

Case 2: $\quad z(e)<0$. Similar to Case 1.

The no-trade rule $\varphi^{0}$ (see Section 4.6, page 104), the hierarchical rule $\varphi^{\text {h }}$ (see Section 4.6, page 104), and the proportional rule $\operatorname{Pr}^{r}$ (see Section 4.2, page 64) establish the independence of the properties in Theorem 5.1.

Loosely speaking, we can interpret Theorem 5.1 as a version of the characterization of the uniform rule $U^{r}$ in Theorem 4.1 by Pareto optimality, strategy-proofness, and no-envy where no-envy is replaced by equal treatment of equals.

The following results of Chapter 4 , which all include the property endowment strategy-proofness, cannot be extended to the model we discuss in this section.

- Corollary 4.3,

- Corollary 4.4,

- Theorem 4.2,

- Theorem 4.11.

In the proof of Corollary 4.3, we use the fact that an agent with individual endowment zero cannot get a negative allotment change. This argument is not valid any more for the model at hand. Since the proofs of Corollary 4.4 and Theorems 4.2 and 4.11 are based on Corollary 4.3, these proofs do not extend to this model either.

All results of Chapter 4 that were not mentioned yet, remain, possibly with small adjustments of notation and definitions, valid for the model discussed in this Section. In case of bilateral consistency (see Section 4.5 , page 98 ) mean leftover changes for the remaining agents are no longer subject to a non-negativity restriction. The same holds for no-envy. This does not affect the proofs.

\subsection{Strategy-proof reallocations without debts}

In Section 5.1 we introduced the reallocation model where the endowments and allotments of the agents may be negative. In this section a variation of this model is considered.

Consider the situation where preferences are still defined on the real line, but where peaks, endowments, and reallocations are restricted to be non-negative. Hence, debts are not allowred any more although agents still have preferences over debts. As an exarmple, in an exchange economy only non-negative endowments, peaks, and reallocations can occur, although agents may have preferences defined on debts. 
The model where we allow the agent's peaks to be negative is essentially the same as the model at hand. All results we derive here can be adjusted to this model by transforming negative peaks to zero and adjusting the definitions of the properties we discuss in the sequel (see Klaus, Peters, and Storcken, 1995b, Section 4.1).

Throughout this section, an economy is formalized as follows. Let $N \in \mathcal{N}$ and $\omega=\left(\omega_{i}\right)_{i \in N} \in \mathbb{R}_{+}^{N}$ be a vector of individual endowments that have to be reallocated among the agents in $N$ with profile $R \in \mathcal{R}_{\mathbb{R}}^{N}$ such that $p(R) \in \mathbb{R}_{+}^{N}$. We denote this class of preferences by $\mathcal{R}_{\mathbb{R}_{1}, \mathbb{R}_{+}}$. We call a pair $e=(R, \omega) \in \mathcal{R}_{\mathbb{R}, \mathbb{R}_{+}}^{N} \times \mathbb{R}_{+}^{N}$ an economy. Let $\mathcal{E}_{2}^{N}=\mathcal{R}_{\mathbb{R}_{1} \mathbb{R}_{+}} \times \mathbb{R}_{+}^{N}$ be the class of all these economies. A feasible allocation for $e=$ $(R, \omega) \in \mathcal{E}_{2}^{N}$, or a reallocation for short, is a vector $x \in \mathbb{R}_{+}^{N}$ such that $\sum_{N} x_{i}=\sum_{N} \omega_{i}$. A reallocation rule $\varphi$, or a rule for short, is a function that assigns to every $N \in \mathcal{N}$ and every $e=(R, \omega) \in \mathcal{E}_{2}^{N}$ a reallocation, denoted by $\varphi(e)$.

For the model at hand, the definitions of Pareto optimality and strategy-proofness as introduced in Section 5.1 apply by replacing the set of economies $\mathcal{E}_{1}^{N}$ by the set of economies $\mathcal{E}_{2}^{N}$. Furthermore, Lemmas 5.1 and 5.2 remain valid. Note that by restricting peaks, individual endowments, and reallocations to be non-negative, we again introduce an asymmetry into the reallocation model (see also Chapter 4, Remark 4.2).

With the next reversibility property we introduce a notion of symmetry into the model. Suppose an economy is "reversed" in the sense that each agent's peak and individual endowment are interchanged, and each agent's preference relation is reflected in his peak and translated to his individual endowment. Then, reversibility requires that in such a reversed economy the allotment changes in one economy equal the reversed allotment changes of the other economy. To formalize this condition we need the following notation.

Let $R_{i} \in \mathcal{R}_{\mathbb{R}_{1}, \mathbb{R}_{+}}$. Then $R_{i}^{r} \in \mathcal{R}_{\mathbb{R}_{,} \mathbb{R}_{+}}$is the reflection of $R_{i}$ (in the peak $p\left(R_{i}\right)$ ), if for all $\alpha, \beta \in \mathbb{R}, \alpha R_{i}^{r} \beta$ if $\left(2 p\left(R_{i}\right)-\alpha\right) R_{i}\left(2 p\left(R_{i}\right)-\beta\right)$. By $R^{r}:=\left(R_{i}^{r}\right)_{i \in N}$ we denote the reflection of $R \in \mathcal{R}_{\mathbb{R}_{1} \mathbb{R}_{+} \text {. }}$

Let $N \in \mathcal{N}$ and $e=(R, \omega) \in \mathcal{E}_{2}^{N}$. Then, the corresponding reversed economy with vector of individual endowments $p(R)$ and preference profile $\left(R^{r}-(p(R)-\omega)\right)$ is denoted by $e^{r}$. So, in the reversed economy (a "reversal" or "reflection" of the original economy) the role of individual endowments and peaks is interchanged whereby each preference relation is reflected in the peak and translated from the peak to the individual endowment. By this reversal, all agents demand (supply) at $e$ as much as they supply (demand) in the reversed economy $e^{r}$. With the following condition we link the outcome of an economy to the outcome of the corresponding reversed economy.

Reversibility: For all $N \in \mathcal{N}$, all $e=(R, \omega) \in \mathcal{E}_{2}^{N}$ and the corresponding reversed economies $e^{r}$, and all $i \in N, \Delta \varphi_{i}(e)=-\Delta \varphi_{i}\left(e^{r}\right)$.

It is easy to check, that the uniform rule $U^{r}$ satisfies reversibility.

Since, reversibility introduced above and the reversibility condition of Chapter 4 
(see Section 4.3, page 78) are introduced for different reallocation models; they are not comparable. However, one can easily adjust the reversibility condition of Chapter 4 to this model. Then, reversibility we introduced above is a weaker condition (for each economy only a single economy is considered to be the reversed economy).

The main result of this section is the following characterization of the uniform rule $U^{r}$ by Pareto optimality, strategy-proofness, equal treatment of equals, and reversibility.

Theorem 5.2 The uniform rule $U^{r}$ is on the domain of economies $\bigcup_{N \notin \mathcal{N}} \mathcal{E}_{2}^{N}$ the only rule that satisfies Pareto optimality, strategy-proofness, equal-treatment of equals, and reversibility.

Proof. The uniform rule $U^{r}$ satisfies Pareto optimal, strategy-proofness, equal treatment of equals, and reversibility.

To prove the remaining part of the theorem let $\varphi$ be a rule that satisfies the properties listed in the theorem. By Lemmas 5.1 and 5.2 both $\varphi$ and $U^{r}$ satisfy own-peak monotonicity and uncompromisingness.

Let $N \in \mathcal{N}, e=(R, \omega) \in \mathcal{E}_{2}^{N}$, and suppose, by contradiction, that $\varphi(e) \neq U^{r}(e)$.

Since $\varphi$ is Pareto optimal it follows that either $z(e)>0$ or $z(e)<0$. By reversibility it is sufficient to consider the case $z(e)>0$. For this case the proof is similar to the proof of Theorem 5.1, Case 1.

Comparing Theorem 5.2 with Theorem 5.1, we only added reversibility to take care of the "asymmetric" excess supply case. This is done, because by applying the proof technique of the excess demand case, the lower bound zero for the peaks might be tight, i.e., possibly we are not able to shift agents towards the maximal supply, because we hit the lower bound. However, by reversibility, the excess supply case is "reversed" to the excess demand case, where the lower bound in not tight. Instead of adding the property of reversibility, one may also replace equal-treatment of equals by no-envy; in other words, Theorem 4.1 also holds in this context.

Remark 5.1 By going over the proofs the reader may verify that our results, in particular Theorems 5.1 and 5.2 remain valid if the domain of single-peaked preferences is replaced by the much smaller domain of all single-peaked preferences that are symmetric around their peaks or that linearly depend only on the distance to the peaks. It is interesting to note that there is a trade-off between this domain restriction and strategy-proofness: on the one hand the restriction of the preference domain weakens strategy-proofness (less preference profiles can be used in unilateral deviations). On the other hand by the domain restriction extra information about rules is implied, e.g., when preferences are symmetrical they are completely described by their peaks. Consequently, on this domain a rule satisfies peak-onliness (see Section 4.3, page 79 ) by definition.

Remark 5.2 Comparing our characterization of the uniform reallocation rule with Ching's characterization of the uniform allocation rule (see Theorem 2.4), we observe that the equal-treatment of equals condition we impose is not logically equivalent 
with Ching's equal treatment of equals. In addition, we impose a reversibility property which guarantees a symmetrical treatment of excess demand and excess supply economies. Now, imposing translation invariance, i.e, translating an economy along the non-negative real line has no impact on the allotment changes, would compensate the asymmetry between excess demand and excess supply in this reallocation setting as well. Then, we can translate any economy to an economy where all agents have identical individual endowments. In this case, Pareto optimality, strategy-proofness, and equal-treatment of equals are logically equivalent to the corresponding properties for allocation economies and the uniform allocation equals the uniform reallocation. As a consequence, Ching's result can be straightforwardly applied and by translation invariance, we obtain the uniform reallocation for the initial economy. With a similar argument, Sprumont's characterizations of the uniform allocation rule (see Theorem 2.1) by Pareto optimality, strategy-proofness, and anonymity directly imply corresponding characterizations of the uniform reallocation rule.

All results of Chapter 4 that were not mentioned yet, remain, possibly with small adjustments of notation and definitions, valid for the model discussed in this section.

\subsection{Further model variations of preferences, endow- ments, and the set of agents}

\subsubsection{Discontinuous preferences}

The first model variation we consider in this section concerns the domain of the preferences. We assumed until now, that the preferences of the agents are single-peaked and continuous. In fact continuity is only needed to prove Lemma 4.8 . Whether the results based on this lemma, Corollaries 4.3 and 4.4 , and Theorem 4.2 , hold true for the whole class of single-peaked preferences is not yet clear. Continuity is also used in the proof of Lemma 4.19. However, this proof can easily be adjusted.

\subsubsection{Bounded peaks, individual endowments, and realloca- tions}

In Chapter 4 we introduced the reallocation model where preferences are defined over the non-negative real line and peaks, endowments, and reallocations are non-negative. In the next model variation, we assume that endowments, peaks, and reallocations are not only bounded from below by zero, but that they are also bounded from above.

Of course, properties like no-envy, weak no-envy, and bilateral consistency must be adapted to this model in order to guarantee that the (adjusted) endowments, which are used in these conditions, are well defined. Then, similarly to Example 4.1, we can construct a counterexample that shows that Corollaries 4.1 and 4.2 do not remain valid. 


\subsubsection{Finite potential set of agents}

Up to now, we assumed that the set of potential agents is infinite. This assumption is crucial for the proofs of Theorems 4.4 and 4.5. As already mentioned in Section 4.5 , Theorem 4.9 and Corollary 4.11 only remain true for a set of potential agents that contain at least three agents. Lemma 4.21 and Theorem 4.10 only remain true for a set of potential agents that contain at least three agents. All other results remain true because the proofs of these theorems apply to a fixed number of agents. 


\section{Appendix A: Allocation of an indivisible good}

The allocation model where an indivisible commodity is allocated among a group of agents is similar to the allocation model with single-dipped preferences we introduced in Chapter 3 . In order to keep this appendix self-contained, we introduce the whole model and restate the central properties we explore. However, for more details concerning the motivation and the background of the properties we analyze here, we refer to Chapters 2 and 3 .

There is a finite population of potential agents, indexed by $\mathbb{P}$. By $\mathcal{P}$ we denote the class of non-empty subsets of $\mathbb{P} . A n$ indivisible commodity $\Omega$ has to be allocated among a group of agents. Each agent $i \in \mathbb{P}$ is equipped with a preference relation $R_{i}$ defined ower the two alternatives "receiving nothing", denoted by 0 , and "receiving the indivisible commodity", denoted by $\Omega$. Hence, for each agent $i$ either $0 R_{i} \Omega$, or $\Omega R_{i} 0$. $\mathbb{B y} \mathcal{R}_{\{0, \Omega\}}$ we denote the set of preference relations over $\{0, \Omega\}$.

For $N \in \mathcal{P}, \mathcal{R}_{\{0, \Omega\}}^{N}$ denotes the set of (preference) profiles $R=\left(R_{i}\right)_{i \in N}$ such that for all $i \in N, R_{i} \in \mathcal{R}_{\{0, \Omega\}}$.

Now, an economy is formalized as follows. Let $\Omega$ be the indivisible commodity that has to be allocated among a group of agents $N \in \mathcal{P}$ with profile $R \in \mathcal{R}_{\{0, \Omega\}{ }^{2}}{ }^{2}$ We call such a pair $e=(R, \Omega)$ an economy. The set of all these economies is denoted by $\mathcal{E}_{\{0, \Omega)}^{N}$.

Remark A.1 The allocation model introduced above is more general than the allocation model introduced by Papai (1996). In Papai (1996) the set of agents is fixed, agents preferences are such that they are not indifferent between 0 and $\Omega$, and there is free disposial of the indivisible comodity.

Let $N \in \mathcal{P}$ and let $X^{N}=\left\{\chi^{i}\right\}_{i \in N}$ denote the unit vectors in $\mathbb{R}^{N}$, where $\chi_{j}^{i}=1$ if $j=i$ and $\chi_{j}^{i}=0$ if $j \neq i$. A feasible allocation for $e=(R, \Omega)$ is an allocation of the indivisible good $\Omega$ to an agent $i \in N$; we denote this allocation by $\Omega \chi^{i}$. Let $\Omega X^{N}=\left\{\Omega \chi^{i}\right\}_{i \in N}$ denote the set of feasible allocations for an economy $e=(R, \Omega)$. An allocation rule $\varphi$, or a rule for short, is a function that assigns to every $N \in \mathcal{P}$ and every $e \in \mathcal{E}_{\{a \Omega\}}^{N}$ a feasible allocation, denoted $\varphi(e)$. Given $i \in N$, we call $\varphi_{i}(e)$ the allotment of agent $i$. Note that either $\varphi_{i}(e)=0$ or $\varphi_{i}(e)=\Omega$.

\footnotetext{
${ }^{2}$ Note that free disposal of the commodity is not allowed.
} 
The first property we are interested in is Pareto optimality.

Pareto optimality: For all $N \in \mathcal{P}$ and all $e=(R, \Omega\} \in \mathcal{E}_{\{0,2\}}^{N}$, there is no feasible allocation $x \in \mathbb{R}_{+}^{N}$, such that for all $i \in N, x_{i} R_{i} \varphi_{i}(e)$, and for some $j \in N, x_{j} P_{j} \varphi_{j}(e)$.

Before we introduce the other properties, we give a simple description of Pareto optimality (see alos Lemma 3.1). For this description we use the partition $\left\{N_{+}(e)\right.$, $\left.N_{0}(e), N_{-}(e)\right\}$ of the agents we introduced in Chapter 3.

Lemma A.1 A rule $\varphi$ is Pareto optimal if and only if for all $N \in \mathcal{P}$ and all $e=$ $(R, \Omega) \in \mathcal{E}_{\{0, \Omega\}}^{N}$, the following holds.

Case 1: If $N_{+}(e) \neq 0$, then

$$
\varphi(e) \in\left\{\Omega \chi^{i} \mid i \in N_{+}(e)\right\} .
$$

Case 2: If $N_{+}(R)=\emptyset$ and $N_{0}(R) \neq \emptyset$, then

$$
\varphi(e) \in\left\{\Omega \chi^{i} \mid i \in N_{0}(e)\right\} \text {. }
$$

Case 3: If $N_{-}(R)=N$, then

$$
\varphi(e) \in\left\{\Omega \chi^{i} \mid i \in N\right\}
$$

In addition to Pareto optimality, we are interested in strategy-proofness (see also Section 2.2, page 22) and replacement-domination (see also Section 2.2, page 24).

Strategy-proofness: For all $N \in \mathcal{P}$, all $e=(R, \Omega) \in \mathcal{E}_{\{0, \Omega\} ;}^{N}$ all $\bar{e}=(\bar{R}, \Omega) \in \mathcal{E}_{\{0 ; \Omega\} \text { : }}^{N}$ and all $j \in N$, if $\bar{R}$ is a $j$-deviation from $R$, then $\varphi_{j}(e) R_{j} \varphi_{j}(\bar{e})$.

Replacement-domination: For all $N \in \mathcal{P}$, all $e=(R, \Omega) \in \mathcal{E}_{\{0, \Omega\}}^{N}$, all $\bar{e}=(\bar{R}, \Omega) \in$ $\mathcal{E}_{\{0, \Omega\}}^{N}$, and all $j \in N$, if $\bar{R}$ is a $j$-deviation from $R$, then either for all $j \in N \backslash\{j\}$; $\left.\varphi_{i}(e) R_{i} \varphi_{i}(\bar{e})\right]$ or $\left[\right.$ for all $\left.i \in N \backslash\{j\}, \varphi_{i}(\bar{e}) R_{i} \varphi_{i}(e)\right]$.

Now, the class of all Pareto optimal, strategy-proof, and replacement dominant rules can be described similarly to the rules described in Chapter 3 , Theorem 3.1 and Corollary 3.1. For a more detailed description of the selection mechanism, we refer the reader to Chapter 3 .

Corollary A.1 A rule $\varphi$ satisfies Pareto optimality, strategy-proofness, and replacement-domination if and only if for all $N \in \mathcal{P}$ there exist $\pi \in \mathbb{T}^{N}, g \in G^{N}$, and $k \in N$ such that for all $e=(R, \Omega) \in \mathcal{E}_{\{0, \Omega\}}^{N}$ :

$$
\varphi(e)=\Omega \chi^{f_{N, 8, k}^{N}(e)} .
$$

Remark A.2 Restricting our model to the model of Papai (1996), the class of rules described in Corollary A.1 equals the serial dictatorships described in Papai (1996). 
Papai (1996) proved that for her model, the set of Pareto optimal, strategy-proof, and "non-bossy" rules equals the set of serial dictatorships.

The next property we consider is consistency (see also Section 2.2, page 26). Consider an economy $e=(R, \Omega) \in \mathcal{E}_{\{0, \Omega\}}^{N}$ and let $\varphi(e)=x$. Suppose that a group of agents leave with their allotments at $x$ and that the agents in $M \subseteq N$ are left behind. If $\Omega=x_{i}$ for $i \in M$, then the reduced economy equals $\left(R_{M}, \Omega\right)$. If $\Omega=x_{i}$ for $i \in N \backslash M$, then the reduced economy equals $\left(R_{M}, 0\right)$. With a slightly abuse of notation, we denote the reduced economies by $\left(R_{M}, \Sigma_{M} x_{i}\right) \in \mathcal{E}^{M}$. Let $M, N \in \mathcal{P}$ such that $M \subseteq N$. For $x \in \Omega X^{N}$ the restriction $\left(x_{i}\right)_{i \in M}$ of $x$ to $M$ is denoted by $x_{M}$.

Consistency: For all $M, N \in \mathcal{P}$ with $M \subseteq N$ and all $e=(R, \Omega) \in \mathcal{E}^{N}, \varphi(e)_{M}=$ $\varphi\left(R_{M}, \sum_{M} \varphi_{i}(e)\right)$.

Next, the class of all Pareto optimal, strategy-proof, and consistent rules can be described similarly to the rules described in Chapter 3, Theorem 3.2. For a more detailed description of the selection mechanism, we refer the reader to Chapter 3.

Corollary A.2 A rule $\varphi$ satisfies Pareto optimality, strategy-proofness, and consistency if and only if there exist $\pi_{+}, \pi_{-} \in \Pi^{\mathbb{P}}$ and $\mathcal{G} \in \mathcal{C}^{\mathbb{P}}$ such that for all $N \in \mathcal{P}$ and all $e=(R, \Omega) \in \mathcal{E}_{\{0, \Omega\}}^{N}$,

$$
\varphi(e)=\Omega \chi^{f_{\pi_{+}, g, \pi_{-}}^{N}(e)}
$$

Finally, we consider population-monotonicity (see also Section 2.2, page 24).

Population-monotonicity: For all $N, M \in \mathcal{P}$, all $e=(R, \Omega) \in \mathcal{E}_{\{0, \Omega\}}^{N}$, and all $\bar{e}=(\bar{R}, \Omega) \in \mathcal{E}_{\{0, \Omega\}}^{N}$, if $M \subseteq N$ and $R_{M}=\tilde{R}$, then either [for all $i \in M, \varphi_{i}(e) R_{i} \varphi_{i}(\bar{e})$ ] or [for all $\left.i \in M, \varphi_{i}(\bar{e}) R_{i} \varphi_{i}(e)\right]$.

Finally the class of all Pareto optimal, strategy-proof, and population-monotonic rules can be described similarly to the rules described in Chapter 3, Theorem 3.3. For a more detailed description of the selection mechanism, we refer the reader to Chapter 3.

Corollary A.3 A rule $\varphi$ satisfies Pareto optimality, strategy-proofness, and population-monotonicity if and only if there exist $\pi_{+}, \pi_{-} \in \Pi^{\mathbb{P}}$ and $\mathcal{G}=\left\{g^{N} \in G^{N}\right\}_{N \in \mathcal{P}}$ such that for all $N \in \mathcal{P}$ and all $e=(R, \Omega) \in \mathcal{E}_{\{0 \Omega\}}^{N}$,

$$
\varphi(e)=\Omega \chi^{f_{\pi_{+}, g, \pi_{-}}^{N}(e)} .
$$

The rule described in Example 3.2, interpreted as a rule for our model, satisfies all properties we considered until now. The rules described in Examples 3.1, 3.4, and 3.5, adjusted for our model, establish the independence of the properties in Corollaries A.1, A.2, and A.3. 


\section{Appendix B: Two alternative proofs}

Alternative proof of Theorem 4.1. The uniform rule $U^{r}$ is same-sided and therefore Pareto optimal. As shown in Lemmas 4.3 and 4.6 it satisfies strategy-proofness and no-envy.

To prove the remaining part of the theorem let $\varphi$ be a rule that satisfies Pareto optimality, strategy-proofness, and no-envy. By Lernma 4.4, both $\varphi$ and $U^{r}$ satisfy individual peak-onliness.

Let $N \in \mathcal{N}, e=(R, \omega) \in \mathcal{E}^{N}$, and suppose, by contradiction, that $\varphi(e) \neq U^{r}(e)$. Since $\varphi$ is Pareto optimal it follows that either $z(e)>0$ or $z(e)<0$. Assume, without loss of generality, that $z(e)<0$. Since $\varphi(e) \neq U^{r}(e)$, there exists an agent $j \in N$ such that $p\left(R_{j}\right)<\varphi_{j}(e)$ and $\Delta \varphi_{j}(e)>\Delta U_{j}^{r}(e)$. Let $\bar{e}=(\bar{R}, \omega) \in \mathcal{E}^{N}$ be such that $\bar{R}$ is a $j$ deviation from $R$ such that $p\left(R_{j}\right)=p\left(\bar{R}_{j}\right)$ and $0 \bar{P}_{j} \varphi_{j}(e){ }^{3}$ By individual peak-onliness it follows that $\varphi_{j}(\bar{e})=\varphi_{j}(e)$ and $U_{j}^{r}(\bar{e})=U_{j}^{r}(e)$. Hence, $\Delta \varphi_{j}(\bar{e})>\Delta U_{j}^{r}(\bar{e})$. Then, by feasibility, there exist $k \in N \backslash\{j\}$ such that $\Delta \varphi_{k}(\bar{e})<\Delta U_{k}^{r}(\bar{e})$. By same-sidedness, this can only be the case if $p\left(\bar{R}_{k}\right)<U_{k}^{r}(\bar{e})$ and $\Delta U_{k}^{r}(\bar{e})=\min \left\{\Delta U_{i}^{r}(\bar{e}) \mid i \in N\right\}$. Thus, $\Delta \varphi_{k}(\bar{e})<\Delta U_{k}^{r}(\bar{e}) \leq \Delta U_{j}^{r}(\bar{e})<\Delta \varphi_{j}(\bar{e})$. So, in contradiction to no-envy, $\left(\omega_{j}+\Delta \varphi_{k}(\bar{e})\right)^{+} \bar{P}_{j} \varphi_{j}(\bar{e})$.

Alternative proof of Theorem 4.5. By same-sidedness and Lemmas 4.6 and 4.12 it follows that the uniform rule satisfies Pareto optimality, no-envy, and one-sided replacement-domination. Furthermore, the uniform rule is replication-invariant.

To prove the remaining part of the theorem let $\varphi$ be a rule that satisfies the properties listed in the theorem. Let $N \in \mathcal{N}, e=(R, \omega) \in \mathcal{E}^{N}$, and suppose, by contradiction, that $\varphi(e) \neq U^{r}(e)$. Since $\varphi$ is Pareto optimal it follows that either $z(e)>0$ or $z(e)<0$. Assume, without loss of generality, that $N=\{1,2, \ldots, n\}$ and $z(e)<0$. Since $\varphi(e) \neq$ $U^{r}(e)$, there exists an agent $j \in N$ such that $p\left(R_{j}\right)<\varphi_{j}(e)$ and $\Delta \varphi_{j}(e)>\Delta U^{r}(e)$.

Let $N^{\prime}=\{n+1, \ldots, 2 n\}$ and define $e^{r}=\left(R^{\prime}, \omega^{\prime}\right) \in \mathcal{E}^{N \cup N^{\prime}}$ such that for all $i \in N$, $\omega_{i}=\omega_{i}^{\prime}=\omega_{n+i}^{\prime}$ and $R_{i}=R_{i}^{\prime}=R_{i+n}^{\prime}$. Clearly, $e^{\prime}$ is a 2-replica of e. Hence, by replication-invariance, for all $i \in N, \varphi_{i}(e)=\varphi_{i}\left(e^{\prime}\right)=\varphi_{n+i}\left(e^{\prime}\right)$. Particularly, it follows that $p\left(R_{j}\right)<\varphi_{j}\left(e^{\prime}\right)$ and $\Delta \varphi_{j}\left(e^{\prime}\right)>\Delta U_{j}^{r}\left(e^{\prime}\right)$.

Let $\bar{R} \in \mathcal{R}^{N N^{\prime}}$ be a $j$-deviation from $R^{\prime}$ such that $p\left(R_{j}^{\prime}\right)=p\left(\bar{R}_{j}\right)$ and $0 \bar{P}_{j} \varphi_{j}\left(e^{\prime}\right)$. Let $\bar{e}=\left(\bar{R}_{n}, \omega^{\prime}\right)$ denote this one-sided change of economy $e^{*}$. Since $p\left(\bar{R}_{j}\right)=p\left(\bar{R}_{n+j}\right)$ and $\omega_{j}^{\prime}=\omega_{n+j}^{\prime}$, by same-sidedness and no-envy, it follows that $\varphi_{j}(\bar{e})=\varphi_{n+j}(\bar{e})$. Suppose

\footnotetext{
${ }^{3}$ In the proof of the excess demand case, let $\widetilde{e}=\left(\widetilde{R}_{,} \omega\right) \in \mathcal{E}^{N}$ be such that $R$ is a $j$-dieviation from $R$ such that $p\left(R_{j}\right)=p\left(\bar{R}_{j}\right)$ and $\sum_{\mathbb{N}} \omega_{i} \bar{P}_{j} \varphi_{j}(e)$.
} 
that $\varphi_{j}(\bar{e})<\varphi_{j}\left(e^{\prime}\right)$. Hence, $\varphi_{n+j}(\bar{e})<\varphi_{n+j}\left(e^{\prime}\right)$ and, by same-sidedness and one-sided replacement-domination, for all $i \in N \backslash\{j\} \cup N^{\prime}, \varphi_{i}(\bar{e}) \leq \varphi_{i}\left(e^{f}\right)$. Then, by feasibility, it follows that $\sum_{N U N^{\prime}} \omega_{i}^{\prime \prime}=\sum_{N U N^{\prime}} \varphi_{i}(\bar{e})<\sum_{N U N^{\prime}} \varphi_{i}\left(e^{\prime}\right)=\sum_{N U N^{\prime}} \omega_{i}^{\prime}$. This is a contradiction. Similarly, the assumption, $\varphi_{j}(\bar{e})>\varphi_{j}\left(e^{\prime}\right)$ yields a contradiction. Hence, $\varphi_{j}(\vec{e})=\varphi_{j}\left(e^{\prime}\right)$. Then, by same-sidedness and one-sided replacement-domination, for all $i \in N \backslash\{j\} \cup N^{\prime}, \varphi_{i}(\vec{e})=\varphi_{i}\left(e^{r}\right)$. Hence, $\varphi(\bar{e})=\varphi\left(e^{\prime}\right)$. Then, $\Delta \varphi_{j}(\vec{e})>\Delta U_{j}^{r}(\bar{e})$ and by feasibility, there exist $k \in N \backslash\{j\} \cup N^{\prime}$ such that $\Delta \varphi_{k}(\bar{e})<\Delta U_{k}^{r}(\bar{e})$. By same-sidedness, this can only be the case if $p\left(\bar{R}_{k}\right)<U_{k}^{r}(\bar{e})$ and $\Delta U_{k}^{r}(\bar{e})=\min \left\{\Delta U_{i}^{r}(\bar{e}) \mid i \in N \cup N^{\prime}\right\}$. Thus, $\Delta \varphi_{k}(\bar{e})<\Delta U_{k}^{r}(\bar{e}) \leq \Delta U_{j}^{r}(\bar{e})<\Delta \varphi_{j}(\bar{e})$. So, in contradiction to no-envy, $\left(\omega_{j}+\Delta \varphi_{k}(\bar{e})\right)+\bar{P}_{j} \varphi_{j}(\tilde{e})$. 


\section{References}

Aumann, R.J., and M. Maschler (1985): "Game Theoretic Analysis of a Bankruptcy" Problem from the Talmud", Journal of Economic Theory 36, 195-213.

Barberà, S., F. Gul, and E. Stacchetti (199): "Generalized Median Voter Schemes and Committees", Journal of Economic Theory 61, 262-289.

Barberà, S., and M.O. Jackson (1995): "Strategy-Proof Exchange", Econometrica 63, $51-87$.

Barberà, S., M.O. Jackson, and A. Neme (1997): "Strategy-Proof Allotment Rules", Games and Economic Behavior 18, 1-21.

Benassy, J.P. (1982): The Economics of Market Disequilibrium, San Diego: Academic Press.

Black, D. (1948): "On the Rationale of Group Decision Making", Journal of Political Economy 56, 23-34.

Blin, J.M., and M.A. Satterthwaite (1976): "Strategy-Pronfness and Single Peakedness", Public Choice 26, 26-51.

Border, K.C., and J.S. Jordan (1983): "Straightforward Elections, Unanimity and Phantom Voters", Review of Economic Studies 50, 153-170.

Ching, S. (1992): "A Simple Characterization of the Uniform Rule", Economics Letters $40,57-60$.

Ching, S. (1994): "An Alternative Characterization of the Uniform Rule", Social Choice and Welfare 11, 131-136.

Dagan, N, and O. Volij (1993): "The Bankruptcy Problem: A Cooperative Bargaining Approach", Mathematical Social Sciences 26, 287-297.

Dagan, N. (1996): "A Note on Thomson's Characterization of the Uniform Rule", Joumal of Economic Theory 69, 255-261.

Dummett, M., and R. Farquharson (1961): "Stability in Voting", Econometrica 29, $33-44$ 
Dutta, B., and D. Ray (1989): "A Concept of Egalitarianism under Participation Constraints", Econometrica 57,615-635.

Foley, D. (1967): "Resource Allocation and the Public Sector", Yale Economic Essays $7,45-98$.

De Frutos, M.A., and J. Massó (1995): "More on the Uniform Rule: Equality and Consistency", Working Paper, Boston University and Universitat Autònoma de Barcelona.

Gibbard, A. (1973): "Manipulation of Voting Schemes: A General Result", Econometrica $41,587-601$.

Hilbert, D. (1918): "Axiomatisches Denken", Mathematische Annalen 78, 405-415.

Hokari, T. (1997): "Weighted Dutta-Ray Solutions on Convex Games", Working Paper, University of Rochester.

Hurwicz, L. (1972): "On Informationally Decentrallized Systems", Decision and Organization, eds. B. McGuire and R. Radner, Amsterdam, North-Holland, 297-336.

Inada, K.I. (1964): "A Note on the Simple Majority Decision Rule", Econometrica 32, $525-531$.

Klaus, B. (1994): "Kooperatieve Spieltheorie: Geometrischer und Algebraischer Vergleich von Spielklassen und Duale Characterisierungen", Diplomarbeit in Mathematik, Mathematische-Naturwissenschaftliche Fakultät der Rheinisch-Westfälischen Technischen Hochschule Aachen.

Klaus, B. (1997a): "The Independence of Replication-Invariance: Two Answers Concerning the Problem of Fair Division when Preferences are Single-Peaked", Working Paper, Maastricht University.

Klaus, B. (1997b): "The Characterization of the Uniform Reallocation Rule without Pareto optimality", "Game Theoretical Applications to Economics and Operations Research, eds. T. Parthasarathy, B. Dutta, J.A.M. Potters, T.E.S. Raghavan, D. Ray, and A. Sen, Dordrecht, Kluwer-Academic Publishers, 239-255.

Klaus, B. (1997c): "Compatibilities and Incompatibilities in Economies with SingleDipped Preferences and the Allocation of an Indivisible Grood", Working Paper, Maastricht University.

\footnotetext{
"For some reason, the publishers changed the original title of the paper from "Individual Rationality and Endowment Monotonicity imply Pareto Optimality; Two Characterizations of the Uniform Reallocation Rulle" to the following two titles. In the table of contents the article is cited as "The characterisation of the Uniform Reallocation Rule Without Pareto optimality". The printed version of the article obtained the title "The Characterisation of the Uniform Reallocation Rule without Side Payments".
} 
Klaus, B. (1997d): "More on the Uniform Reallocation Rule: Summary and Strengthening of former Results", Working Paper, Maastricht University.

Klaus, B., H. Peters, and T. Storcken (1995a): "Strategy-Proof Division with SinglePeaked Preferences and Individual Endowments", Social Choice and Welfare, forthcoming.

Klaus, B., H. Peters, and T. Storcken (1995b): "Strategy-Proof Reallocation of an Infinitely Divisible Good", Charlemagne and his Heritage: 1200 Years of Civilization and Science in Europe, Volume 2: Mathematical Arts, eds. P.L. Butzer, H.Th. Jongen, and W. Oberschelp, Aachen, Thouet Verlag, forthcoming.

Klaus, B., H. Peters, and T. Storcken (1997a): "Strategy-Proof Division of a Private Good when Preferences are Single-Dipped", Economics Letters 55, 339-346.

Klaus, B., H. Peters, and T. Storcken (1997b): "Reallocation of an Infinitely Divisible Good", Economic Theory 10, 305-333.

Klaus, B., and T. Storcken (1994): "Voting Correspondences for Public Goods", METEOR Research Memorandum RM/0/94-050, Maastricht University.

Mas-Colell, A. (1992): "Equilibrium Theory with Possibly Satiated Preferences", Equilibrium and Dynamics, Essays in Honour of D. Gale, ed. M. Majumdar, London, McMillan, 201-213.

Mas-Colell, A., M.D. Whinston, and J.R. Green (1995): Microeconomic Theory, New York, Oxford University Press.

Moreno, B. (1996): "The Uniform Rule in Economies with Single-Peaked Preferences, Endowments and Population-Monotonicity", Working Paper, University of Alicante.

Moulin, H. (1980): "On Strategy-Proofness and Single Peakedness", Public Choice 35, $437-455$.

Moulin, H. (1987a): "Equal or Proportional Division of a Surplus, and other Methods", International Journal of Game Theory 16, 161-186.

Moulin, H. (1987b): "The Pure Compensation Problem: Egalitarianism versus LaissezFairism", Quarterly Journal of Economics 102, 769-783.

Moulin, H. (1997): "Rationing and Additive Cost Sharing", Game Theory and Resource Allocation: The Axiomatic Approach (NATO Advanced Study Institute, Stony Brook, 1997), Springer Verlag, forthcoming.

Otten, G.-J., H. Peters, and O. Volij (1996): "Two Characterizations of the Uniform Rule for Division Problems with Single-Peaked Preferences"s" Economic Theory 7, $291-306$. 
Papai, S. (1996): "Strategyproof Allocation of a Single-Object". Social Science Working Paper 936, California Institute of Technology.

Peremans, W., and T. Storcken (1997): "Strategy-Proof Decisions on Public Bads", Working Paper, Mastricht University.

Postlewaite, A. (1979): "Manipulation via Endowments", Review of Economic Studies $46,255-262$.

Satterthwaite, M.A. (1975): "Strategy-Proofmess and Arrow's Conditions: Existence and Correspondence Theorems for Voting Procedures and Social Welfare Functions", Journal of Economic Theory 10, 187-217.

Schmeidler, D., and K. Vind (1972): "Fair Net Trades", Econometrica 40, 637-642.

Schummer, J., and W. Thomson (1997): "Two Derivations of the Uniform Rule and an Application to Bankruptcy", Economics Letters 55, 333-337.

Sönmez, T. (1994): "Consistency, Monotonicity and the Uniform Rule", Economics Letters $46,229-235$.

Sprumont, Y. (1991): "The Division Problem with Single-Peaked Preferences: A Characterization of the Uniform Allocation Rule", Econometrica 59, 509-519.

Thomson, W. (1983): "The Fair Division of a Fixed Supply Among a Growing Population", Mathematics of Operations Research 8 "319-326.

Thomson, W. (1990): "Manipulation and Implementation of Solutions to the Problem of Fair Division when Preferences are Single-Peaked", Working Paper, University of Rochester, revised April 1992.

Thomison, W. (1993): "The Replacement Principle in Public Good Economies with Single-Peaked Preferences", Economics Letters 42, 31-36.

Thomson, W. (1994a): "Resource-Monotonic Solutions to the Problem of Fair Division when Preferences are Single-Peaked", Social Choice and Welfare 11, 205-223.

Thomson, W. (1994b): "Consistent Solutions to the Problem of Fair Division when Preferences are Single-Peaked", Journal of Economic Theory 63, 219-245.

Thomson, W. (1995a): "Population-Monotonic Solutions to the Problen of Fair Division when Preferences are Single-Peaked", Economic Theory 5, 229-246.

Thomson, W. (1995b): "Population Monotonic Allocation Rules" , Social Choice, Welfare and Ethics, eds. W.A. Barnett, H. Moulin, M. Salles, and N. Schofield, Cambridge University Press.

Thomson, W. (1995c): "Axiomatic Analysis of Bankruptcy and Taxation Problems: A Survey", Working Paper, University of Rochester. 
Thomson, W. (1995d): "Endowment-Monotonicity in Economies with Single-Peaked Preferences", Working Paper, University of Rochester, revised May 1996.

Thomson, W. (1996a): "The Replacement Principle in Economies with Indivisible Goods", Social Choice and Welfare, forthcoming.

Thomson, W. (1996b): Consistent Allocation Rules, Fundamentals of Pure and Applied Economics, Horwood Academic Publishers, forthcoming.

Thomson, W. (1997a): "The Replacement Principle in Economies with Single-Peaked Preferences", Journal of Economic Theory 76, 145-168.

Thomson, W. (1997b): The Theory of Fair Allocation, Princeton University Press, forthcoming.

Vickrey, W. (1960): "Utility, Strategy and Social Decision Rules", Quarterly Journal of Economics 75, 507-535.

Young, P. (1987): "On Dividing an Amount According to Individual Claims or Liabilities", Mathematics of Operations Research 12, 398-414.

Young, P. (1988): "Distributive Justice in Taxation", Journal of Economic Theory 44, 321-335. 


\section{Index}

\section{Author Index}

A

Aristotle 19

Aumann 8, 20

B

Barberà $7-8,12,57,65,139$

Benassy $8,17,22,57,64,66,138$

Black 7

Blin 7

Border 7,68

$\mathrm{C}$

Ching $10,17,19,22-23,59,67-68,70$, 117

D

Dagan 10,17, 20,27,61, 99, 101-103

De Frutos 10, 21

Dummett 7

Dutta 21

F

Farquharson 7

Foley 22,69

G

Gibbard 7, 137

Green 48

Gull 7

H

Hurwicz 8, 137

I

Inada 11,35
J

Jackson $8,12,57,65,139$

Jordan 7,68

K

Klaus $17,35,44,57,84,89,93,109,116$

M

Mas-Colell 48,64

Maschler 8, 20

Massó 10, 21

Moreno $65,79,82-84$

Moulin 7-8, 20, 23

$\mathbf{N}$

Neme 12, 57, 65, 139

$\mathrm{O}$

O'Neill 8

Otten 10, 21

$\mathbf{P}$

Papai 43, 121-122

Peremans 11, 35

Peters 10, 21, 35, 44, 57, 84, 89, 93, 109, 116

Postlewaite 73

R

Ray 21

$\mathrm{S}$

Sönmez 10, 17, 19, 28, 101

Satterthwaite 7, 137

Schmeidler 69

Schummer 20,64

Sprumont 10, 17, 19, 21-22, 30, 52, 57, $59,66,70,77,118,138$ 


\section{Stacchetti 7}

Storcken 11, 35, 44, 57, 84, 89, 93, 109, 116

$\mathbf{T}$

Thomson $10,17-28,61,64-65,79,81$, $83,89-90,93-95,97,102,138$

V

Vickrey $\mathbb{1 1}, 35$

Vind 69

Volij 10, 20-21

W

Whinston 48

$\mathbf{Y}$

Young $8,19-20$ 


\section{Subject Index}

\section{A}

absorbing agent rule 30,105

adjusted endowment vector 97

allocation of teaching hours 8

allocation rule $18,36,44$

allotment 62,110

allotment change 62,110

anonymity $10,22,51,77$

B

balancedness 62,110

bankruptcy 20

bargaining 21

beer allocation 9

beer reallocation 12

bilateral consistency 27,98

bounded preferences 18

boundedness by endowments and peaks 90,92

broken allocation 39,48

broken share 40

C

choice function 41

consistency $26,45,97,123$

consistent collection 46

converse consistency 28,52

D

demand 62,110

demand-onliness 76

demander 62,110

$\operatorname{dip} 36$

dip-onliness 51

dummy property 90-91

$\mathbf{E}$

economy $18,36,44,62,110,116$

endowment continuity 27

endowment strategy-proofness 73

endowment-monotonicity 81,88

equal treatment of equals $10,23,52,72$, excess demand $8,20,62,110$

excess supply $8,20,62,110$

F

fair allocation 8

fair reallocation 8

feasible allocation $18,36,44,62,110$, 116

G

game theory 21

H

hierarchical rule 104

I

im-deviation $21,39,66,73,111$

i-deviation from withholding 73

individual endowment 61,110

individual peak-onliness 68,112

individual rationality 92

individual rationality from equal division 27,51

indivisible commodity 121

$\mathbf{K}$

$k$-replica 25,86

$\mathbf{L}$

linear order 41

M

maximal demand $70,91,114$

maximal demander 70,114

maximal supplier 71

maximal supply 71

maximally satiating rule 105

merged economy 82

$\mathbf{N}$

no-envy $8,22,52,69,113$

no-trade rule 104

non-satiated agent 62,110

non-trader 62,110 
O

one-sided change 24,74

one-sided endowment-monotonicity 88 one-sided population-monotonicity 24,82 one-sided replacement-domination 24,85 one-sided resource-monotonicity 24 one-sided welfare-domination under preference-replacement 24

own-peak monotonicity 67,112

$\mathbf{P}$

Pareto optimality $19,37,63,111,122$ partition 25,85

peak $18,61,110$

peak-onliness 22,79

permutation $22,41,77$

population-monotonicity $8,23,48,81-$ 82,123

preference function 18,61

preference profile $18,36,44,62,110$

proportional (reallocation) rule 64

proportional (allocation) rule 19

\section{$\mathbf{R}$}

reallocation $62,110,116$

reallocation of teaching hours 12

reallocation rule $62,110,116$

reduced economy $26,45,98$

reflection 116

replacement-domination $39,84,122$

replication-invariance $25,52,86$

resource-monotonicity 23

restriction $21,26,39,45,66,73,111$

reversed economy 116

reversibility 78,116

$\mathrm{S}$

same-sidedness $19,63,111$

satiated agent 62,110

selection 41

share $19,37,44,62$

single-dipped preferences 10,36

single-peaked preferences $18,7,61,110$

social endowment 18,44 strategy-proofness $7,22,39,66,111$

strong one-sided endowmentmonotonicity 89

supplier 62,110

supply 62,110

$\mathrm{T}$

taxation 20

total demand 62,110

total supply 62,110

translation 113

$\mathrm{U}$

uncompromisingness 68,112

uniform (allocation) rule $8,19,35$

uniform (reallocation) rule $12,64,111$

V

vector of dips 51

vector of peaks $18,62,110$

\section{W}

weak no-envy 70

welfare-domination under preferencereplacement $23,81,84$ 
List of Symbols

$\mathcal{A}^{N} 29$

Bं 39

$\mathcal{B}_{q} 48$

$\mathcal{D} 36$

$\mathcal{E}^{N} 18,44,62$

$\mathcal{E}_{1}^{N} 110$

$\varepsilon_{2}^{N} 116$

$\mathcal{E}_{b}^{N} 18$

G 46

$\mathcal{N}_{18,62}$

P 44

$\mathcal{R} 18,62$

$\mathcal{R}_{\mathrm{b}} 18$

$\mathcal{X} 36$

$\Omega 18,44$

$\omega(i, j) 97$

$\omega \cup \bar{\omega} 82$

$\omega_{i} 61,110$

$\pi 22,41,77$

$\Pi^{N} 22,77$

$\tau 113$

$\Delta \varphi_{i}(e) 62,110$

$\varphi^{a} 30$

$d(e) 62,110$

$d\left(R_{i}\right) 36$

$d_{i}(e) 62,110$

e $18,44,62,110,116$

eบ e 82

$e^{r} 116$

$g 41$

$N_{+}(R) 37$

$N_{-}(R) 37$

$N_{0}(R) 37$

Pr 19

$\operatorname{Pr}^{*} 64$

$p(R) 18,62,110$

$p\left(R_{i}\right) 61,110$

$R 18,36,44,62,110$

$R \cup \tilde{R} 82$

$r_{i} 61$

$R_{N} 21,66,111$

$s(e) 62,110$ $s_{i}(e) 62,110$

$U 19$

$U^{r} 64,111$

X 36

$x_{M} 45$

$z(e) 62$ 


\section{Summary in Dutch}

\section{Eerlijke Verdeling en Herverdeling: Een Axiomatische Studie}

In tal van economische en politieke situaties zijn de preferenties van de betrokken agenten privé-informatie. Afhankelijk van het gekozen beslissingsmechanisme, kunnen agenten een reden hebben om hun ware voorkeuren te verbergen, om zodoende een betere uitkomst te bewerkstelligen, mogelijk ten koste van anderen. Om dit soort gedrag te vermijden kunnen beslissingsmechanismen gekozen worden die "strategy-proof" oftewel niet-manipuleerbaar zijn. Dit houdt in dat geen enkele agent ooit een voordeel kan hebben van het opzettelijk foutief weergeven van zijn preferenties, ongeacht de preferenties die geuit worden door de overige agenten.

Het eerste fundamentele resultaat met betrekking tot niet-manipuleerbaarheid van Gibbard (1973) en Satterthwaite (1975) is dat, als alle preferenties toelaatbaar zijn, in essentie alleen dictatoriale mechanismen niet-manipuleerbaar kunnen zijn. Echter, niet-dictatoriale mechanismen kunnen wel degelijk niet-manipuleerbaar zijn als niet alle preferenties worden toegelaten. Een vocrbeeld is de beperking tot de klasse van "singlepeaked" preferenties: preferenties zijn single-peaked als alle mogelijke alternatieven op een dusdanige manier geordend kunnen worden, dat er voor iedere agent een uniek optimum is, de piek, en dat zijn voorkeur monotoon daalt in beide richtingen van de piek af.

\section{Verdeel Modellen}

Gedurende een lange periode bleven alle bijdragen op het gebied van de analyse van niet-manipuleerbare mechanismen beperkt tot publieke goederen. In dit proefschrift beschouwen we een aantal modellen, waarin een oneindig deelbaar privaat goed verdeeld of herverdeeld wordt onder een aantal agenten. Niet-manipuleerbaarheid is daarbij een centraal thema. Maar we zijn ook geinteresseerd in zaken als eerlijkheid ("fairness") en solidariteit, bijvoorbeeld "no-envy" of populatie-monotoniciteit. No-envy wil zeggen dat agenten nooit het aandeel van andere agenten prefereren boven hưn eigen aandeel. Populatie-monotoniciteit houdt in dat indien er een nieuwe agent opduikt, alle andere agenten daar allemaal (zwak) beter, of allemaal (zwak) slechter wan worden. In dit proefschrift betitelen we de analyse van mechanismen die voldoen aan eerlijkheid en andere "gewenste" eigenschappen als "fair allocation" oftewel eerlijke verdeling.

Ook de literatuur over niet-manipuleerbaarheid in economieën met private goederen begint met een onmogelijkheidsstelling. Voor ruileconomieën met twee agenten en twee goederen, toont Hurwicz (1972) aan dat er zelfs voor de beperkte klasse van "trans- 
lated" Cobb-Douglas preferenties, in essentie geen verdeelmechanismen bestaan die nietmanipuleerbaar en Pareto optimaal zijn. Net als voor de publieke goederen houdt dit resultaat niet stand voor sommige andere klassen van preferenties, zoals de klasse van single-peaked preferenties. Een typisch voorbeeld is het verdelen van twee goederen in een ruileconomie waarin vraag en aanbod niet in evenwicht zijn (zie Benassy, 1982). In deze context van niet-manipuleerbare ruil beschrijft Benassy (1982) wat nu bekend staat als de uniforme regel. Deze regel verdeelt het totaal over de agenten zo "gelijk mogelijk", door de pieken van de agenten als bovengrenzen te nemen in geval van een tekort en als ondergrenzen in geval van een overschot. De uniforme regel kan ook toegepast worden voor het verdelen van een "social endowment" van een oneindig deelbaar goed over een groep van agenten met single-peaked preferenties, bijvoorbeeld de onderwijslast van een vakgroep over zijn docenten.

Het blijkt dat de uniforme regel aan tal van gewenste eigenschappen voldoet, zoals Pareto optimaliteit en niet-manipuleerbaarheid. Bovendien is het de enige regel die voldoet aan deze eigenschappen en no-envy of anonimiteit (zie Sprumont 1991). Tevens voldoet de uniforme regel aan eigenschappen als consistentie, solidariteit, en monotoniciteit (zie bijvoorbeeld Thomson 1997b). Een conclusie van dit onderzoek is dat de uniforme regel nu geaccepteerd is als de belangrijkste regel voor problemen waarbij een eerlijke verdeling gewenst is en waarbij de preferenties single-peaked zijn.

\section{HOOFDSTUK 2: Eerlijke Verdeling en Single-Peakedness}

In Hoofdstuk 2, geven we een overzicht van de meest belangrijke resultaten met betrekking tot de uniforme verdeelregel. Ook geven we antwoorden op twee open vragen gesteld door William Thomson in verband met twee karakteriseringen van de uniforme regel (Thomson 1995a, 1997a): door middel van een voorbeeld geven we een positief antwoord op de vraag of "replication-invariance" in deze karakteriseringen onafhankelijk is van de andere eigenschappen.

Na Hoofdstuk 2 vervolgen we met twee varianten het "klassieke" verdelingsmodel met single-peaked preferenties. In de eerste variant beschouwen we het probleem van eerlijke verdeling voor een klasse van preferenties die omschreven kan worden als "omgekeerd single-peaked", namelijk "single-dipped" preferenties. Preferenties zijn singledipped als alle mogelijke alternatieven op een dusdanige manier geordend kunnen worden, dat er voor iedere agent een uniek minst geprefereerd punt is, de "dip", en dat zijn voorkeur monotoon stijgt in beide richtingen van de dip af. In de tweede variant gaat het om een verdelingsmodel met single-peaked preferenties, waarbij we aannemen dat een sociaal goed herverdeeld moet worden, in plaats van verdeeld.

\section{HOOFDSTUK 3: Eerlijke Verdeling en Single-Dippedness}

Beschouw het verdelen van twee soorten werk met negatieve kruiseffecten, bijvoorbeeld onderwijzen en besturen aan een universiteit: combinaties van beide soorten werk zijn mogelijk minder geprefereerd dan werk van alleen één soort. Zo'n situatie kan geformaliseerd worden als een verdeelprobleem met single-dipped preferenties. Een ander voorbeeld is een twee-goederen ruileconomie met vaste prijzen en strikt quasi-convexe nutsfuncties, welke single-dipped preferenties geven op de budgetlijnen.

De aanname dat preferenties single-dipped in plaats van single-peaked zijn, blijkt bij 
problemen van eerlijke verdeling een belangrijke rol te spelen. Het blijkt namelijk dat één van de belangrijkste eigenschappen, namelijk Pareto optimaliteit, nooit samengaat met eigenschappen van eerlijkheid, zoals anonimiteit en no-enwy. Bovendien blijkt dat het totale goed aan één enkele agent toegewezen moet worden om te voldoen aan de eigenschappen Pareto optimaliteit, niet-manipuleerbaarheil, aangevuld met of consistentie of een solidariteit eigenschap (of populatie-monotoniciteit of "replacement-domination"). Tevens geven we een volledige karakterisering van alle verdeelregels die voldoen aan de bovenstaande combinaties van eigenschappen. Het is opmerkelijk dat de karakteriseringen ook voor verdeelproblemen met een ondeelbaar goed geldig zijn.

\section{HOOFDSTUKKEN 4 en 5: Eerlijke Herverdeling en Single-Peakedness}

In economische situaties zoals de eerder genoemde ruileconomie ontstaan individuele bezittingen op een natuurlijke wijze. Daarom is een logische uitbreiding van het model van eerlijke verdeling met single-peaked preferenties, de introductie van individuele bezittingen. Een andere interpretatie van deze uitbreiding wordt gegeven in Barberà, Jackson, en Neme (1997). Zij beginnen vanuit het oorspronkelijke verdelingsmodel met single-peaked preferenties en laten anonimiteit buiten beschouwing door het introduceren van rechten van agenten en rangordes van agenten, bijvoorbeeld in investeringssituaties.

Net als voor het oorspronkelijke model van eerlijke verdeling, zijn we geïnteresseerd in een axiomatische analyse van eerlijke herverdeling en in het bijzonder in een uitbreiding van de uniforme regel naar het herverdelingsmodel: de uniforme herverdeelregell. Net als de uniforme verdeelregel voldoet de uniforme herverdeelregel aan gewenste eigenschappen zoals Pareto optimaliteit, niet-manipuleerbaarheid, en no-envy in termen van de netto veranderingen van de bezittingen. In Hoofdstukken 4 en 5 geven we antwoord op de vraag in hoeverre de resultaten verkregen voor het verdelingsmodel gegeneraliseerd kunnen worden naar het herverdelingsmodel. In Hoofdstuk 4 introduceren en verkennen we het herverdelingsmodel en eigenschappen voor herverdeelregels. Bovendien leiden we enkele karakteriseringen af voor de uniforme herverdeelregel en gaan we in op de overeenkomsten en de verschillen met verwante verdeelresultaten. In Hoofdstuk 5 , tenslotte, bekijken we de gevoeligheid van deze resultaten voor variaties van de model parameters. We laten bijvoorbeeld zien dat de meeste resultaten geldig blijven wanneer we toestaan dat agenten schulden hebben. 


\section{Curriculum Vitae}

Bettina-Elisabeth Klaus werd geboren op 9 februari 1968 te Koblenz (Duitsland). Van 1978 tot 1987 volgde zij het voorbereidend wetenschappelijk onderwijs aan het Staatliche Gymnasium auf dem Asterstein te Koblenz. Na het behalen van het Abitur begon zij in oktober 1987 met de studie wiskunde aan de Rheinisch-Westfälische Technische Hochschule Aachen (Duitsland). Deze studie werd in februari 1994 met de titel Diplom Mathematikerin afgesloten. Bij het schrijven van haar doctoraalscriptie "Kooperative Spieltheorie: Geometrischer und algebraischer Vergleich von Spielklassen und duale Characterisierungen" werd ze begeleid door Prof.dr. Hans Peters en Dr. Jean Derks.

Per 1 november 1993 trad zij in dienst als assistent in opleiding bij de vakgroep Kwantitatieve Economie van de huidige Universiteit Maastricht, waar zij onder de leiding van Prof.dr. H. Peters en Dr. T. Storcken gedurende vier jaar onderzoek verrichtte op het gebied van de sociale keuzetheorie. De meeste resultaten van dit onderzoek zijn weergegeven in dit proefschift.

Sinds 15 januari 1997 is zij als visiting scholar te gast aan het Department of Economics van de University of Rochester, USA. In het najaar 1997 verzorgde ze daar de cursus "Mathematical Economics" voor Ph.D. studenten. 